\title{
Water-Quality, Bed-Sediment, and Biological Data (October 2002 through September 2003) and Statistical Summaries of Data for Streams in the Upper Clark Fork Basin, Montana
}

By Kent A. Dodge, Michelle I. Hornberger, and Irene R. Lavigne

In cooperation with the

U.S. Environmental Protection Agency

Open-File Report 2004-1340

U.S. Department of the Interior

U.S. Geological Survey 


\section{U.S. Department of the Interior}

\section{Gale A. Norton, Secretary}

\section{U.S. Geological Survey Charles G. Groat, Director}

U.S. Geological Survey, Reston, Virginia: 2004

For sale by U.S. Geological Survey, Information Services

Box 25286, Denver Federal Center

Denver, CO 80225

For additional information write to:

District Chief

U.S. Geological Survey

3162 Bozeman Avenue

Helena, Montana 59601

For more information about the USGS and its products:

Telephone: 1-888-ASK-USGS

World Wide Web: http://www.usgs.gov/

Any use of trade, product, or firm names in this publication is for descriptive purposes only and does not imply endorsement by the U.S. Government.

Although this report is in the public domain, permission must be secured from the individual copyright owners to reproduce any copyrighted materials contained within this report. 


\section{CONTENTS}

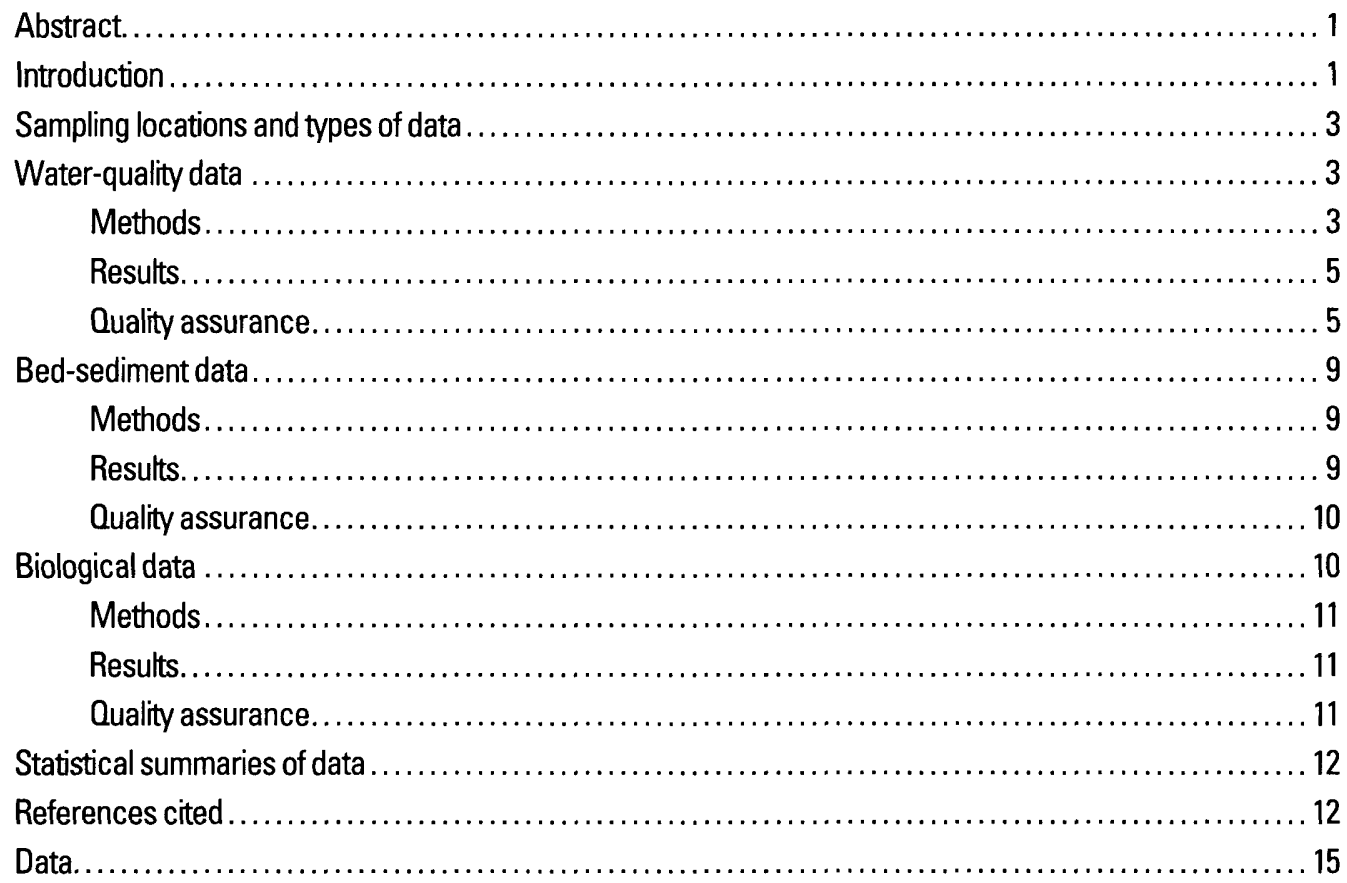

\section{FIGURE}

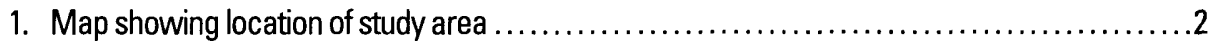

\section{TABLES}

1. Type and period of data collection at sampling stations in the upper Clark Fork basin,

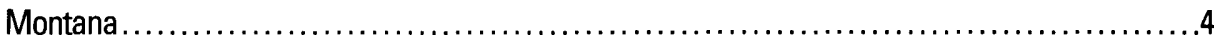

2. Properties measured onsite and constituents analyzed in samples of water, bed sediment, and biota from the upper Clark Fork basin, Montana........................... 5

3. Data-quality objectives for analyses of water-quality samples collected in the

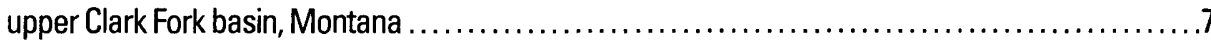

4. Water-quality data for the upper Clark Fork basin, Montana, October 2002 through

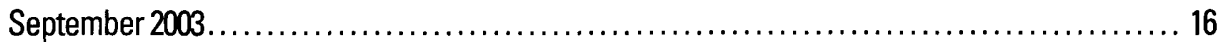

5-7. Daily streamflow and suspended-sediment data, October 2002 through September 2003, for:

5. Clark Fork at Deer Lodge, Montana ............................................... 36

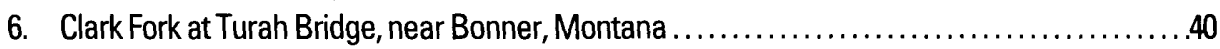

7. Clark Fork above Missoula, Montana ........................................ 44

8. Chemical and suspended-sediment analyses of field replicates for water samples, upper

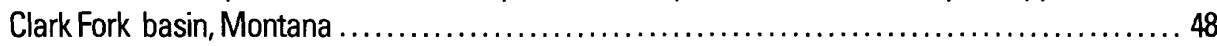

9. Precision of chemical and suspended-sediment analyses of field replicates for water samples, upper Clark Fork basin, Montana. 
10. Precision of chemical analyses of laboratory replicates for water samples, upper Clark

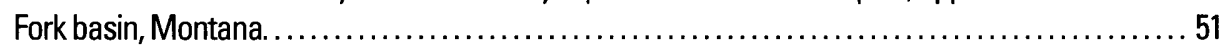

11. Recovery efficiency for trace-element analyses of laboratory-spiked deionized-water blanks. ... 52

12. Recovery efficiency for trace-element analyses of laboratory-spiked stream samples, upper

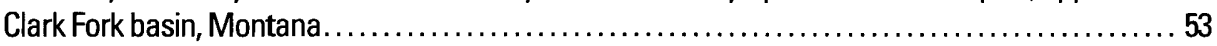

13. Chemical analyses of field blanks for water samples.................................. 54

14. Trace-element analyses of fine-grained bed sediment, upper Clark Fork basin, Montana,

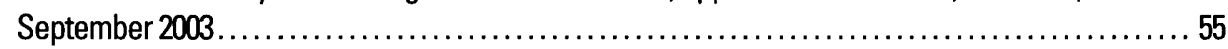

15. Trace-element analyses of bulk bed sediment, upper Clark Fork basin, Montana,

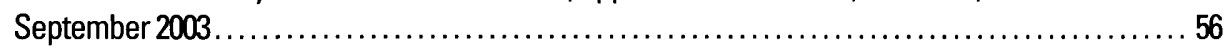

16. Recovery efficiency for trace-element analyses of standard reference materials for bed

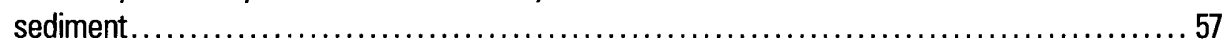

17. Trace-element analyses of procedural blanks for bed sediment ....................... 58

18. Trace-element analyses of biota, upper Clark Fork basin, Montana, September 2003............ 59

19. Recovery efficiency for trace-element analyses of standard reference material for biota. ........61 61

20. Trace-element analyses of procedural blanks for biota .................................. 62

21. Statistical summary of water-quality data for the upper Clark Fork basin, Montana, March

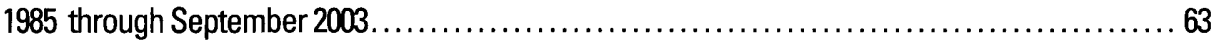

22. Statistical summary of fine-grained bed-sediment data for the upper Clark Fork basin, Montana,

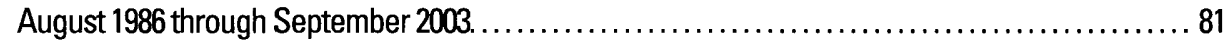

23. Statistical summary of bulk bed-sediment data for the upper Clark Fork basin, Montana,

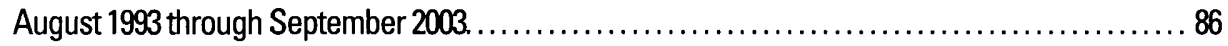

24. Statistical summary of biological data for the upper Clark Fork basin, Montana, August 1986 through September 2003 


\section{Conversion Factors, Datum, Abbreviated Water- Quality Units, and Acronyms}

\begin{tabular}{lcl}
\hline Multiply & By & To obtain \\
\hline cubic foot per second $\left(\mathrm{ft}^{3} / \mathrm{s}\right)$ & 0.02832 & cubic meter per second $\left(\mathrm{m}^{3} / \mathrm{s}\right)$ \\
foot (ft) & 0.3048 & meter $(\mathrm{m})$ \\
gallon (gal) & 3.785 & liter $(\mathrm{L})$ \\
gallon (gal) & 3,785 & milliliter $(\mathrm{mL})$ \\
inch (in.) & 25.4 & millimeter $(\mathrm{mm})$ \\
inch (in.) & 25,400 & micrometer $(\mu \mathrm{m})$ \\
mile (mi) & 1.609 & kilometer \\
ounce (oz) & 28.35 & gram $(\mathrm{g})$ \\
part per million & 1 & microgram per gram $(\mu \mathrm{g} / \mathrm{g})$ \\
square mile (mi $\left.{ }^{2}\right)$ & 2.59 & square kilometer \\
ton per day (ton/d) & 907.2 & kilogram per day \\
\hline
\end{tabular}

Temperature in degrees Celsius $\left({ }^{\circ} \mathrm{C}\right)$ may be converted to degrees Fahrenheit $\left({ }^{\circ} \mathrm{F}\right)$ as follows:

$$
{ }^{\circ} \mathrm{F}=\left(1.8 \mathrm{x}^{\circ} \mathrm{C}\right)+32
$$

Vertical coordinate information is referenced to the National Geodetic Vertical Datum of 1929 (NGVD 29). Horizontal coordinate information is referenced to the North American Datum of 1927 (NAD 27).

Abbreviated water-quality units used in this report:

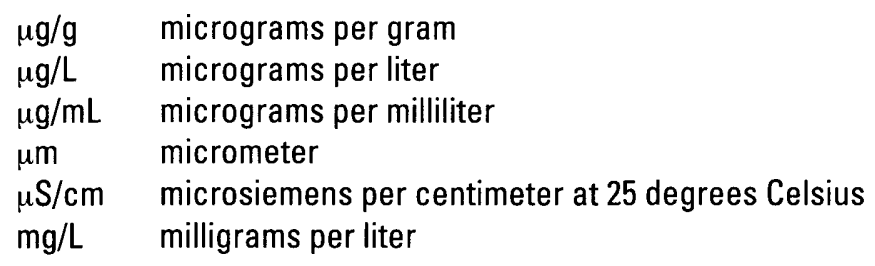

Water-year definition:

A water year is the 12-month period from October 1 through September 30. It is designated by the calendar year in which it ends.

\section{Acronyms used in the report:}

ICAPES inductively coupled argon plasma emission spectroscopy

LRL laboratory reporting level

LT-MDL long-term method detection level
RSD relative standard deviation SRM standard reference material USGS U.S. Geological Survey

NWQL USGS National Water Quality Laboratory, Denver, Colo. 


\title{
Water-Quality, Bed-Sediment, and Biological Data (October 2002 through September 2003) and Statistical Summaries of Data for Streams in the Upper Clark Fork Basin, Montana
}

\author{
By Kent A. Dodge, Michelle I. Hornberger ${ }^{1}$, and Irene R. Lavigne ${ }^{1}$
}

\section{Abstract}

Water, bed sediment, and biota were sampled in streams from Butte to below Missoula as part of a program to characterize aquatic resources in the upper Clark Fork basin of western Montana. Sampling stations were located on the Clark Fork, five major tributaries, and four smaller tributaries. Water-quality data were obtained periodically at 18 stations during October 2002 through September 2003 (water year 2003). Data for 15 bed-sediment and 15 biological stations were obtained in September 2003. The primary constituents analyzed were trace elements associated with tailings from historical mining and smelting activities.

Water-quality data include concentrations of selected major ions, trace elements, and suspended sediment in stream samples. Daily values of streamflow, suspended-sediment concentration, and suspended-sediment discharge are given for three stations. Bed-sediment data include trace-element concentrations in the fine-grained and bulk fractions. Biological data include trace-element concentrations in whole-body tissue of aquatic benthic insects. Quality-assurance data are reported for analytical results of water, bed sediment, and biota. Statistical summaries of water-quality, bed-sediment, and biological data are provided for the period of record since 1985 for each station.

\section{Introduction}

The Clark Fork originates near Warm Springs in western Montana at the confluence of Silver Bow and Warm Springs Creeks (fig. 1). Along the 148-mi reach of stream from Silver Bow Creek in Butte to the Clark Fork at Milltown Reservoir, five major tributaries enter: Warm Springs Creek, Little Blackfoot River, Flint Creek, Rock Creek, and Blackfoot River. Principal surface-water uses in the $6,000-\mathrm{mi}^{2}$ Clark Fork basin above Missoula include irrigation, stock watering, light industry, hydroelectric power generation, and habitat for trout fisher- ies. Current land uses primarily are cattle production, logging, mining, and recreation. Large-scale mining and smelting were prevalent land uses in the upper basin for more than one hundred years, but are now largely discontinued.

Deposits of copper, gold, silver, and lead ores were extensively mined, milled, and smelted in the drainages of Silver Bow and Warm Springs Creeks from about 1870 to 1980. Moderate- and small-scale mining also occurred in the basins of most of the major tributaries to the upper Clark Fork. Tailings derived from mineral processing commonly contain large quantities of trace elements such as arsenic, cadmium, copper, lead, and zinc. Tailings have been eroded, mixed with stream sediment, transported downstream, and deposited in stream channels, on flood plains, and in the Warm Springs Ponds and Milltown Reservoir. The widely dispersed tailings continue to be re-eroded, transported, and redeposited along the stream channel and flood plain, especially during high flows. The occurrence of elevated trace-element concentrations in water and bed sediment can pose a potential risk to aquatic biota and human health.

Concern about the potential toxicity of trace elements to aquatic biota and human health has resulted in a comprehensive effort by State, Federal, and private entities to characterize the aquatic resources in the upper Clark Fork basin to guide and monitor remedial cleanup activities. A long-term data base was considered necessary to detect trends over time in order to evaluate the effectiveness of remediation. Water-quality data have been collected by the U.S. Geological Survey (USGS) at selected sites in the upper Clark Fork basin since 1985 (Lambing, 1987, 1988, 1989, 1990, 1991; Lambing and others, 1994, 1995; and Dodge and others, 1996, 1997, 1998, 1999, 2000, 2001, 2002, 2003). Trace-element data for bed sediment and biota (aquatic benthic insects) have been collected intermittently since 1986 at selected sites as part of studies on bed-sediment contamination and bioaccumulation of metals conducted by the USGS National Research Program (Axtmann and Luoma, 1991; Cain and others, 1992, 1995; Axtmann and others, 1997; Hornberger and others, 1997). In March 1993, an expanded sampling program for water, bed sediment, and biota 
2 Water-Quality, Bed-Sediment, and Biological Data (October 2002 through September 2003) and Statistical Summaries of Data for Streams in the Upper Clark Fork Basin, Montana

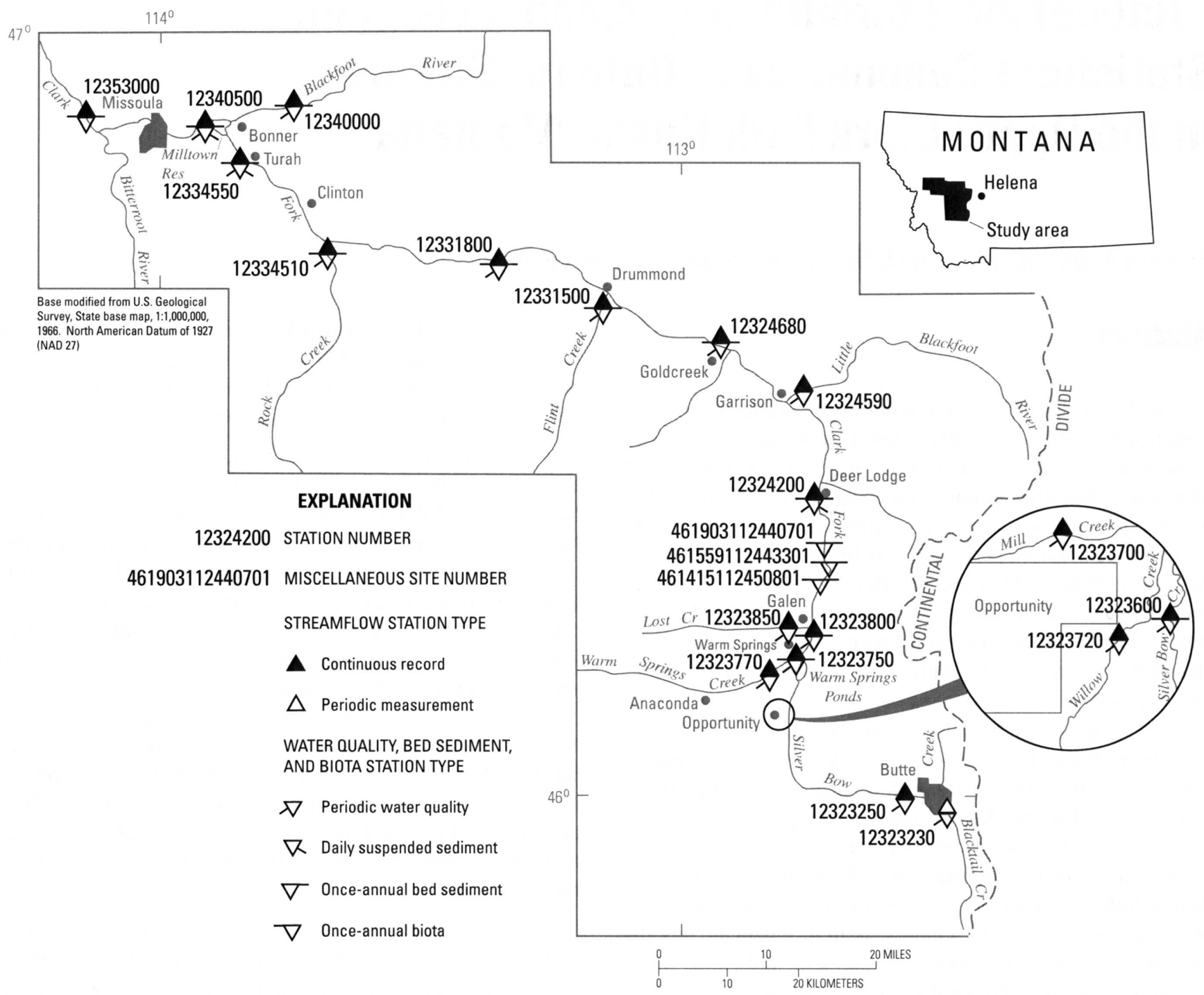

Figure 1. Location of study area. 
was implemented by the USGS in cooperation with the U.S. Environmental Protection Agency to provide systematic, longterm monitoring to better quantify the seasonal and annual variability in selected constituents.

The purpose of this report is to present water-quality data for 18 stations and trace-element data for 15 bed-sediment and 15 biological stations in the upper Clark Fork basin collected from October 2002 through September 2003 (water year 2003). Quality-assurance data are presented for water-quality, bedsediment, and biota samples. Statistical summaries also are provided for water-quality, bed-sediment, and biological data collected since 1985 .

\section{Sampling Locations And Types Of Data}

Sampling stations in the upper Clark Fork basin are located on the Clark Fork mainstem, five major tributaries (Warm Springs Creek at Warm Springs, Little Blackfoot River near Garrison, Flint Creek near Drummond, Rock Creek near Clinton, and Blackfoot River near Bonner), and four smaller tributaries (Blacktail Creek at Harrison Avenue, Mill Creek at Opportunity, Willow Creek at Opportunity, and Lost Creek near Galen) from Butte to below Missoula (fig. 1). The stations, types of data collected, and period of record for each data type are listed in table 1. Mainstem sampling sites were selected to divide the upper Clark Fork into reaches of relatively uniform length, with each reach encompassing either a major tributary or depositional environment (Warm Springs Ponds and Milltown Reservoir). Major tributaries were sampled to describe waterquality characteristics of important hydrologic sources in the upper basin and to provide reference comparisons to the mainstem for bed sediment and biota. In 2003, three additional tributaries were included for continuous streamflow and periodic water-quality sampling - Mill Creek, Willow Creek, and Lost Creek. These tributaries drain the area near Anaconda and were added to gain more resolution on sources of metals entering the Clark Fork in an area of historical metal-processing activities. Water-quality data were obtained periodically at 18 stations; daily suspended-sediment data were obtained for part of the year at 3 of these stations. Trace-element data for 15 bed-sediment and 15 biological stations were obtained once-annually. Continuous streamflow data were collected at 18 stations.

A list of properties measured onsite and constituents analyzed in samples of water, bed sediment, and biota is given in table 2. Results of analyses for water, bed sediment, and biota as well as associated quality-assurance data for water year 2003 are listed in tables 4 through 20 at the back of the report. Statistical summaries of water-quality, bed-sediment, and biological data collected between March 1985 and September 2003 are given in tables 21-24 at the back of the report.

Quality assurance of data was maintained through the use of documented procedures designed to provide environmentally representative data. Acceptable performance of the procedures was verified with quality-control samples that were collected systematically to provide a measure of the accuracy, precision, and bias of the environmental data and to identify problems associated with sampling, processing, or analysis.

\section{Water-Quality Data}

Water-quality data consist of measurements of physical properties and concentrations of chemical and physical constituents analyzed in stream samples. At 14 sites, samples were collected 8 times per year on a schedule designed to describe seasonal and hydrologic variability. At four tributary sites, samples were collected only twice during the water year because of termination, and then reinstatement, of those sites in the sampling program.

\section{Methods}

Cross-sectional water samples were collected from multiple verticals across the stream using depth- and width-integration methods described by Ward and Harr (1990) and Edwards and Glysson (1999). These methods provide a vertically and laterally discharge-weighted composite sample that is representative of the entire flow passing through the cross section of a stream. Sampling equipment consisted of standard USGS depth-integrating suspended-sediment samplers (DH-48, DH81 , and D-74TM), which were either constructed of plastic or coated with a non-metallic epoxy paint, and equipped with nylon or Teflon nozzles.

Instantaneous streamflow at the time of water sampling was determined at all stations, either by direct measurement or from stage-discharge rating tables (Rantz and others, 1982). Onsite measurements of specific conductance, $\mathrm{pH}$, and water temperature were made during collection of periodic waterquality samples. Onsite sample processing, including filtration and preservation, was performed according to procedures described by Ward and Harr (1990), Horowitz and others (1994), and the USGS (variously dated) National Field Manual for the Collection of Water-Quality Data.

Water samples were analyzed for the constituents listed in table 2 by the USGS National Water Quality Laboratory (NWQL) in Denver, Colo. The trace elements (arsenic, cadmium, copper, iron, lead, manganese, and zinc) were analyzed for filtered $(0.45-\mu \mathrm{m}$ pore size $)$ and unfiltered recoverable concentrations. Calcium and magnesium also were determined in filtered samples to enable calculation of hardness. The terms "filtered" and "unfiltered recoverable" replace the terms "dissolved" and "total recoverable," respectively, which were used in the past. Filtered concentrations of arsenic, cadmium, copper, lead, manganese, and zinc were analyzed by inductively coupled plasma-mass spectrometry (Faires, 1993; Garbarino, 1999). Filtered concentrations of calcium, magnesium, and iron were analyzed by inductively coupled plasma-atomic emission spectrometry (Fishman, 1993). Unfiltered-recoverable traceelement concentrations were determined from unfiltered 
Table 1. Type and period of data collection at sampling stations in the upper Clark Fork basin, Montana

[Abbreviation: P, present. Symbol: --, no data]

\begin{tabular}{|c|c|c|c|c|c|c|c|}
\hline $\begin{array}{c}\text { Station number } \\
\text { (fig. 1) }\end{array}$ & Station name & $\begin{array}{c}\text { Continuous- } \\
\text { record } \\
\text { streamflow }\end{array}$ & $\begin{array}{c}\text { Periodic water } \\
\text { quality }^{1}\end{array}$ & $\begin{array}{c}\text { Daily suspended } \\
\text { sediment }\end{array}$ & $\begin{array}{l}\text { Fine-grained } \\
\text { bed sediment }{ }^{2}\end{array}$ & Bulk bed sediment ${ }^{2}$ & Biota $^{2}$ \\
\hline 12323230 & $\begin{array}{l}\text { Blacktail Creek at Harrison Avenue, at } \\
\text { Butte }\end{array}$ & -- & $\begin{array}{l}03 / 93-08 / 95 \\
12 / 96-08 / 03\end{array}$ & -- & -- & -- & -- \\
\hline 12323250 & $\begin{array}{l}\text { Silver Bow Creek below Blacktail Creek, } \\
\text { at Butte }\end{array}$ & 10/83-P & $\begin{array}{l}03 / 93-08 / 95 \\
12 / 96-\mathrm{P}\end{array}$ & -- & -- & -- & - \\
\hline 12323600 & Silver Bow Creek at Opportunity & $07 / 88-\mathrm{P}$ & $\begin{array}{l}\text { 03/93-08/95, } \\
12 / 96-\mathrm{P}\end{array}$ & $03 / 93-09 / 95$ & $07 / 92-\mathrm{P}$ & $\begin{array}{l}08 / 93-08 / 95 \\
08 / 97-\mathrm{P}\end{array}$ & $\begin{array}{l}07 / 92,08 / 94, \\
08 / 95,08 / 97-\mathrm{P}\end{array}$ \\
\hline 12323700 & Mill Creek at Opportunity & $04 / 03-P$ & $03 / 03-\mathrm{P}$ & -- & -- & -- & -- \\
\hline 12323720 & Willow Creek at Opportunity & 04/03-P & $03 / 03-\mathrm{P}$ & - & - & -- & -- \\
\hline 12323750 & Silver Bow Creek at Warm Springs & $\begin{array}{l}03 / 72-09 / 79 \\
04 / 93-P\end{array}$ & $03 / 93-\mathrm{P}$ & $04 / 93-09 / 95$ & 07/92-P & $\begin{array}{l}08 / 93, \\
08 / 95-P\end{array}$ & $07 / 92-\mathrm{P}$ \\
\hline 12323770 & Warm Springs Creek at Warm Springs & 10/83-P & 03/93-P & -- & $\begin{array}{l}08 / 95,08 / 97 \\
08 / 99,08 / 02\end{array}$ & $\begin{array}{l}08 / 95,08 / 97, \\
08 / 99,08 / 02\end{array}$ & $\begin{array}{c}08 / 95,08 / 97,08 / 99 \\
08 / 02\end{array}$ \\
\hline 12323800 & Clark Fork near Galen & $07 / 88-\mathrm{P}$ & $07 / 88-\mathrm{P}$ & -- & $\begin{array}{l}08 / 87, \\
08 / 91-\mathrm{P}\end{array}$ & 08/93-P & $\begin{array}{c}\text { 08/87, } \\
08 / 91-\mathrm{P}\end{array}$ \\
\hline 12323850 & Lost Creek near Galen & $04 / 03-\mathrm{P}$ & $03 / 03-\mathrm{P}$ & -- & -- & -- & $\ldots$ \\
\hline \begin{tabular}{|l|}
461415112450801 \\
\end{tabular} & Clark Fork below Lost Creek, near Galen & -- & -- & -- & $08 / 96-\mathrm{P}$ & $08 / 96-\mathrm{P}$ & $08 / 96-\mathrm{P}$ \\
\hline 461559112443301 & Clark Fork near Racetrack & - & -- & $\ldots$ & $08 / 96-\mathrm{P}$ & $08 / 96-\mathrm{P}$ & $08 / 96-\mathrm{P}$ \\
\hline 461903112440701 & $\begin{array}{l}\text { Clark Fork at Dempsey Creek diversion, } \\
\text { near Racetrack }\end{array}$ & - & -- & - & 08/96-P & $08 / 96-\mathrm{P}$ & $08 / 96-\mathrm{P}$ \\
\hline 12324200 & Clark Fork at Deer Lodge & 10/78-P & 03/85-P & $\begin{array}{c}03 / 85-08 / 86, \\
04 / 87-03 / 03, \\
08 / 03-\mathrm{P}\end{array}$ & $\begin{array}{l}08 / 86,08 / 87, \\
08 / 90-P\end{array}$ & 08/93-P & $\begin{array}{l}08 / 86,08 / 87, \\
08 / 90-\mathrm{P}\end{array}$ \\
\hline 12324590 & Little Blackfoot River near Garrison & 10/72-P & $03 / 85-\mathrm{P}$ & --- & $\begin{array}{c}08 / 86,08 / 87,08 / 94 \\
08 / 98,08 / 01\end{array}$ & $08 / 94,08 / 98,08 / 01$ & $\begin{array}{l}08 / 87,08 / 94 \\
08 / 98,08 / 01\end{array}$ \\
\hline 12324680 & Clark Fork at Goldcreek & $10 / \overline{77-P}$ & 03/93-P & -- & $07 / 92-\mathrm{P}$ & $\overline{08 / 93-\mathrm{P}}$ & $07 / 92-\mathrm{P}$ \\
\hline 12331500 & Flint Creek near Drummond & $\begin{array}{l}08 / 90-04 / 03, \\
08 / 03-\mathrm{P}\end{array}$ & $03 / 85-\mathrm{P}$ & -- & $\begin{array}{l}08 / 86,08 / 89, \\
07 / 92-\mathrm{P}\end{array}$ & $08 / 93-\mathrm{P}$ & $\begin{array}{l}08 / 86 \\
07 / 92-\mathrm{P}\end{array}$ \\
\hline 12331800 & Clark Fork near Drummond & 04/93-P & $03 / 93-P$ & -- & $\begin{array}{l}08 / 86,08 / 87 \\
08 / 91-P\end{array}$ & $\overline{08 / 93-P}$ & $\begin{array}{c}08 / 86, \\
08 / 91-P\end{array}$ \\
\hline 12334510 & Rock Creek near Clinton & $10 / 72-\mathrm{P}$ & $03 / 85-\mathrm{P}$ & - & $\begin{array}{l}08 / 86,08 / 87,08 / 89 \\
08 / 91-99,08 / 01-\mathrm{P}\end{array}$ & $08 / 93-99,08 / 01-\mathrm{P}$ & \begin{tabular}{|c|}
$08 / 87$ \\
$08 / 91-99,08 / 01-\mathrm{P}$
\end{tabular} \\
\hline 12334550 & Clark Fork at Turah Bridge, near Bonner & $03 / 85-\mathrm{P}$ & $03 / 85-\mathrm{P}$ & $\begin{array}{l}03 / 85-03 / 03 \\
08 / 03-\mathrm{P}\end{array}$ & $\begin{array}{l}08 / 86 \\
08 / 91-\mathrm{P}\end{array}$ & 08/93-P & $\begin{array}{l}08 / 86 \\
08 / 91-\mathrm{P}\end{array}$ \\
\hline 12340000 & Blackfoot River near Bonner & 10/39-P & $03 / 85-\mathrm{P}$ & $\begin{array}{l}07 / 86-04 / 87, \\
06 / 88-09 / 95\end{array}$ & $\begin{array}{c}08 / 86,08 / 87, \\
08 / 91, \\
08 / 93-96,08 / 98-01, \\
09 / 03\end{array}$ & $\begin{array}{c}08 / 93,08 / 94,08 / 99-01 \\
09 / 03\end{array}$ & \begin{tabular}{|c|}
$08 / 86,08 / 87$ \\
$08 / 91,08 / 93$ \\
$08 / 96,08 / 98,09 / 00$ \\
$09 / 03$
\end{tabular} \\
\hline 12340500 & Clark Fork above Missoula & $03 / 29-\mathrm{P}$ & $07 / 86-\mathrm{P}^{3}$ & $\begin{array}{c}07 / 86-04 / 87, \\
06 / 88-01 / 96, \\
03 / 96-03 / 03, \\
08 / 03-\mathrm{P}\end{array}$ & $08 / 97-\mathrm{P}$ & $08 / 97-\mathrm{P}$ & $08 / 97-\mathrm{P}$ \\
\hline 12353000 & Clark Fork below Missoula ${ }^{4}$ & 10/29-P & $03 / 85-09 / 95$ & -- & $\begin{array}{c}08 / 86 \\
08 / 90-\mathrm{P}\end{array}$ & 08/93-P & $\begin{array}{c}08 / 86, \\
08 / 90-\mathrm{P}\end{array}$ \\
\hline
\end{tabular}

${ }^{1}$ Onsite measurements of physical properties and laboratory analyses of selected major ions, trace elements, and suspended sediment. Prior to March 1993, laboratory analyses included only trace elements and suspended sediment, with the exception of Clark Fork below Missoula.

2Laboratory analyses of trace elements.

water-quality data for Clark Fork above Missoula only included suspended-sediment data.

${ }^{4}$ Bed sediment and biota sampled about 30 miles downstream from streamflow-gaging station to conform to previous sampling location. 
Table 2. Properties measured onsite and constituents analyzed in samples of water, bed sediment, and biota from the upper Clark Fork basin, Montana

\begin{tabular}{|c|c|c|c|}
\hline \multicolumn{2}{|c|}{ Water } & \multirow{2}{*}{$\frac{\text { Bed sediment }}{\text { Constituent }}$} & \multirow{2}{*}{$\frac{\text { Biota }}{\text { Constituent }}$} \\
\hline Property & Constituent & & \\
\hline Streamflow & Hardness (calculated) & Arsenic & Arsenic \\
\hline Specific conductance & Calcium & Cadmium & Cadmium \\
\hline $\mathrm{pH}$ & Magnesium & Chromium & Chromium \\
\hline \multirow[t]{8}{*}{ Temperature } & Arsenic & Copper & Copper \\
\hline & Cadmium & Iron & Iron \\
\hline & Copper & Lead & Lead \\
\hline & Iron & Manganese & Manganese \\
\hline & Lead & Nickel & Nickel \\
\hline & Manganese & Silver & Zinc \\
\hline & Zinc & Zinc & \\
\hline & Suspended sediment & & \\
\hline
\end{tabular}

samples that were first digested with dilute hydrochloric acid (Hoffman and others, 1996) and then analyzed for arsenic by graphite furnace-atomic absorption spectrometry (Jones and Garbarino, 1999) and for cadmium, copper, iron, lead, manganese, and zinc by inductively coupled plasma-mass spectrometry (Garbarino and Struzeski, 1998).

Cross-sectional water samples also were collected for analysis of suspended sediment whenever periodic water-quality samples were collected. These samples were analyzed for suspended-sediment concentration and the percentage of suspended sediment mass finer than 0.062-mm diameter (silt size and smaller) by the USGS sediment laboratory in Helena, Mont., according to methods described by Guy (1969) and Lambing and Dodge (1993).

At the three daily suspended-sediment stations (table 1), suspended-sediment samples were collected 2 to 9 times per week for only part of the year (October through March, and August through September) as the result of program modifications. These samples were collected by local contract observers using the depth-integration method at a single vertical near midstream. The samples were analyzed for suspended-sediment concentration and were used to determine daily mean suspended-sediment concentrations according to methods described by Porterfield (1972). Five supplemental water-quality and suspended-sediment samples were collected at Clark Fork at Turah Bridge, near Bonner (12334550) and Clark Fork above Missoula (12340500), from May 29-June 2. These samples were collected to better define variability in trace-element and suspended-sediment concentrations above and below Milltown Reservoir during high flow.

\section{Results}

Water-quality data for samples collected periodically during water year 2003 are presented in table 4 . The types of data include instantaneous streamflow, onsite measurements of water-quality properties, and analytical results for chemical constituents and suspended sediment.

Daily streamflow and available daily suspended-sediment data for water year 2003 at the three daily suspended-sediment stations are given in tables 5 through 7 . Monthly descriptive statistics for each parameter are provided along with totals for the annual discharge of water and suspended sediment.

\section{Quality Assurance}

Quality-assurance procedures used for the collection and field processing of water-quality samples are described by Ward and Harr (1990), Knapton and Nimick (1991), Horowitz and others (1994), Edwards and Glysson (1999), and the USGS (variously dated) National Field Manual for the Collection of Water-Quality Data. Standard procedures used by the NWQL for internal sample handling and quality assurance are described by Friedman and Erdmann (1982), Jones (1987), and Pritt and Raese (1995). Quality-assurance procedures used by the Montana District sediment laboratory are described by Lambing and Dodge (1993).

The quality of analytical results reported for water-quality samples was evaluated by quality-control samples that were submitted from the field and analyzed concurrently in the laboratory with routine samples. These quality-control samples consisted of replicates, spikes, and blanks which provide quantitative information on the precision and bias of the overall field and laboratory process. Each type of quality-control sample was submitted at a proportion equivalent to about 5 percent of the total number of water-quality samples. Therefore, the total number of quality-control samples represented about 15 percent of the total number of water-quality samples.

In addition to quality-control samples submitted from the field, internal quality-assurance practices at the NWQL are per- 
formed systematically to provide quality control of analytical procedures (Pritt and Raese, 1995). These internal practices include analyses of quality-control samples such as calibration standards, standard reference water samples, replicate samples, deionized-water blanks, or spiked samples at a proportion equivalent to at least 10 percent of the sample load. The

NWQL participates in a blind-sample program where standard reference water samples prepared by the USGS Branch of Quality Systems are routinely inserted into the sample line for each analytical method at a frequency proportional to the sample load. The laboratory also participates in external evaluation studies twice-yearly with the U.S. Environmental Protection Agency, the Canadian Center for Inland Water, and the USGS Branch of Quality Systems to assess analytical performance.

Replicate data can be obtained in different ways to provide an assessment of precision (reproducibility) of analytical results. Replicate samples are two or more samples considered to be essentially identical in composition. Replicate samples can be obtained in the field (field replicate) by either repeating the collection process to obtain two or more independent composite samples, or by splitting a single composite sample into two or more subsamples. The individual replicate samples are then analyzed separately. Likewise, a single sample can be analyzed two or more times in the laboratory to obtain a measure of analytical variability (laboratory replicate).

Precision of analytical results for field replicates is affected by numerous sources of variability within the field and laboratory environments, including sample collection, sample processing, and sample analysis. To provide data on precision for samples exposed to all sources of variability, replicate stream samples for chemical analysis were obtained in the field by splitting a composite stream sample, and replicate stream samples for suspended-sediment analysis were obtained in the field by concurrently collecting two independent cross-sectional samples. Analyses of these field replicates indicate the reproducibility of environmental data that are affected by the combined variability potentially introduced by field and laboratory processes.

Analytical precision was evaluated with laboratory replicates, which excluded field sources of variability. Two independent analyses were made of an individual sample selected randomly in the laboratory from the group of samples comprising each analytical run. A separate analysis of the sample was made at the beginning and end of each analytical run to provide information on the reproducibility of laboratory analytical results independent of possible variability caused by field collection and processing of samples.

Spiked samples are used to evaluate bias, which measures the ability of an analytical method to accurately quantify a known amount of analyte added to a sample. Because some constituents in stream water can potentially interfere with the analysis of a targeted analyte, it is important to determine whether such effects are causing biased (inaccurate) analyses. Deionized-water blanks and aliquots of stream samples were spiked in the laboratory with known amounts of the same trace elements analyzed in water samples. Analyses of spiked blanks indicate if the spiking procedure and analytical method are within control for a water matrix that is presumably free of chemical interference. Analyses of spiked aliquots of stream samples indicate if the chemical matrix of the stream water interferes with the analytical measurement and whether these interferences could contribute significant bias to reported traceelement concentrations for stream samples.

Blank samples of deionized water were routinely analyzed to identify the presence and magnitude of contamination that potentially could bias analytical results. The particular type of blank sample routinely tested was a "field" blank. Field blanks are aliquots of deionized water that are certified as traceelement free and are processed through the sampling equipment used to collect stream samples. These blanks are then subjected to the same processing (sample splitting, filtration, preservation, transportation, and laboratory handling) as stream samples. Blank samples are analyzed for the same constituents as those of stream samples to identify whether any detectable concentrations exist.

All water samples were handled in accordance with chainof-custody procedures that provide documentation of sample identity, shipment, receipt, and laboratory handling. All samples submitted from a sampling episode were stored and analyzed as a discrete sample group, independent of other samples submitted to the NWQL. Therefore, statistical descriptions of quality-control data generated for this monitoring program are directly applicable to the analytical results for stream samples reported herein.

Data-quality objectives (table 3 ) were established for water-quality data as part of the study plan for the expanded long-term monitoring program that was initiated in 1993. The objectives identify analytical requirements of detectability and serve as a guide for identifying questionable data by establishing acceptable limits for precision and bias of laboratory results. Comparisons of quality-control data to data-quality objectives are used to evaluate whether sampling and analytical procedures are producing environmentally representative data in a consistent manner. Data that did not meet the objectives were evaluated for acceptability, and corrective action was taken, when appropriate.

During water year 1999, the NWQL began implementation of a new, statistically based convention for establishing reporting levels and for reporting low-concentration data (Childress and others, 1999). Quality-control data are collected on a continuing basis to determine long-term method detection levels (LT-MDLs) and laboratory reporting levels (LRLs). These values are re-evaluated each year and, consequently, may change from year to year. The methods are designed to limit the possible occurrence of a false positive or false negative error to 1 percent or less. Accordingly, concentrations are reported as less than the LRL for samples in which the analyte was either not detected or did not pass identification criteria. Analytes that are detected at concentrations between the LT-MDL and LRL and that pass identification criteria are estimated. Estimated concentrations are noted with a remark code of "E." These data 
need to be used with the understanding that their uncertainty is greater than that of data reported without the "E" remark code.

The precision of analytical results for a constituent can be determined by estimating a standard deviation of the differences between replicate measurements for several sets of samples. These replicate measurements may consist either of individual analyses of a pair of samples considered to be essentially identical (field replicates) or multiple analyses of an individual sample (laboratory replicates). The differences in concentration between replicate analyses can be used to estimate a standard deviation according to the following equation (Taylor, 1987):

$$
S=\sqrt{\frac{\Sigma d^{2}}{2 k}}
$$

where

$S=$ standard deviation of the difference in concentration between replicate analyses,

$d=$ difference in concentration between each pair of replicate analyses, and

$k=$ number of pairs of replicate analyses.

Precision also can be expressed as a relative standard deviation (RSD), in percent, which is computed from the standard deviation and the mean concentration for all the replicate analyses. Expressing precision relative to a mean concentration standardizes comparison of precision among individual constituents. The RSD, in percent, is calculated according to the following equation (Taylor, 1987):

Table 3. Data-quality objectives for analyses of water-quality samples collected in the upper Clark Fork basin, Montana

[The terms "filtered" and "unfiltered recoverable" replace the former terms "dissolved" and "total recoverable," respectively. Abbreviations: $\mu \mathrm{g} / \mathrm{L}$, micrograms per liter; mg/L, milligrams per liter; $\mathrm{mm}$, millimeter. Symbol: --, not determined]

\begin{tabular}{|c|c|c|c|c|}
\hline \multirow[b]{3}{*}{ Constituent } & \multicolumn{4}{|c|}{ Data-quality objectives } \\
\hline & \multicolumn{2}{|c|}{ Detectability } & \multirow{2}{*}{$\begin{array}{c}\text { Precision } \\
\text { Maximum relative } \\
\text { standard deviation of } \\
\text { laboratory replicate } \\
\text { analyses, } \\
\text { in percent }\end{array}$} & \multirow{2}{*}{$\begin{array}{c}\text { Bias } \\
\text { Maximum } \\
\text { deviation of } \\
\text { spike recovery, } \\
\text { in percent }\end{array}$} \\
\hline & $\begin{array}{r}\text { Labor } \\
\text { repo } \\
\text { lev }\end{array}$ & $\begin{array}{l}\text { ratory } \\
\text { rting } \\
\text { el }^{1}\end{array}$ & & \\
\hline Calcium, filtered & 0.01 & $\mathrm{mg} / \mathrm{L}$ & 20 & -- \\
\hline Magnesium, filtered & .008 & $\mathrm{mg} / \mathrm{L}$ & 20 & -- \\
\hline Arsenic, unfiltered recoverable & 2 & $\mu \mathrm{g} / \mathrm{L}$ & 20 & 25 \\
\hline Arsenic, filtered & .3 & $\mu \mathrm{g} / \mathrm{L}$ & 20 & 25 \\
\hline Cadmium, unfiltered recoverable & .04 & $\mu \mathrm{g} / \mathrm{L}$ & 20 & 25 \\
\hline Cadmium, filtered & .04 & $\mu \mathrm{g} / \mathrm{L}$ & 20 & 25 \\
\hline Copper, unfiltered recoverable & .6 & $\mu \mathrm{g} / \mathrm{L}$ & 20 & 25 \\
\hline Copper, filtered & .2 & $\mu \mathrm{g} / \mathrm{L}$ & 20 & 25 \\
\hline Iron, unfiltered recoverable & $6-20$ & $\mu \mathrm{g} / \mathrm{L}$ & 20 & 25 \\
\hline Iron, filtered & $8-10$ & $\mu \mathrm{g} / \mathrm{L}$ & 20 & 25 \\
\hline Lead, unfiltered recoverable & .06 & $\mu \mathrm{g} / \mathrm{L}$ & 20 & 25 \\
\hline Lead, filtered & .08 & $\mu \mathrm{g} / \mathrm{L}$ & 20 & 25 \\
\hline Manganese, unfiltered recoverable & .22 & $\mu g / L$ & 20 & 25 \\
\hline Manganese, filtered & .2 & $\mu \mathrm{g} / \mathrm{L}$ & 20 & 25 \\
\hline Zinc, unfiltered recoverable & 2 & $\mu \mathrm{g} / \mathrm{L}$ & 20 & 25 \\
\hline Zinc, filtered & 1 & $\mu \mathrm{g} / \mathrm{L}$ & 20 & 25 \\
\hline Sediment, suspended & 1 & $\mathrm{mg} / \mathrm{L}$ & -- & -- \\
\hline Sediment, suspended, percent finer than $0.062 \mathrm{~mm}$ & 1 & percent & -- & -- \\
\hline
\end{tabular}

${ }^{1}$ For those constituents showing a range of values, the laboratory reporting level changed during water year 2003. 
8 Water-Quality, Bed-Sediment, and Biological Data (October 2002 through September 2003) and Statistical Summaries of Data for Streams in the Upper Clark Fork Basin, Montana

$$
R S D=\frac{S}{\bar{x}} \times 100
$$

where

$$
\begin{aligned}
& R S D=\text { relative standard deviation, } \\
& S=\text { standard deviation, and } \\
& \bar{x}=\text { mean of all replicate concentrations. }
\end{aligned}
$$

Paired analyses of field replicates are presented in table 8 . The precision estimated for each constituent based on these paired results, which include both field and laboratory sources of variability, is reported in table 9 . Statistics for precision of field-replicate analyses were based on the values reported in table 8 , which are rounded to standard USGS reporting levels for the particular constituent and its analytical method (Timme, 1994).

Data-quality objectives for precision are not directly applicable to field replicates because of the inability to determine whether the variability results from field sample collection and processing, or laboratory handling and analysis. However, a statistical calculation of precision for the field replicates is provided in table 9 to illustrate overall reproducibility of environmental data that incorporates both field and laboratory sources of variability. The data-quality objective used to evaluate precision of results for field replicates was a maximum relative standard deviation of 20 percent (table 3 ). Precision estimates for the field replicate analysis were within the 20 -percent relative standard deviation limit for all constituents.

Analytical precision for chemical constituents based on replicate laboratory analyses of individual samples, which includes only laboratory sources of variability, is reported in table 10. Statistics for analytical precision of laboratoryreplicate analyses are based on unrounded values stored in laboratory data files. The data-quality objective for analytical precision of laboratory-replicate analyses was a maximum relative standard deviation of 20 percent. Precision estimates for laboratory-replicate analyses (table 10) were within the 20 percent relative standard deviation limit for all constituents.

Analyses of an unspiked sample and a spiked aliquot of the same sample provide a measure of the recovery efficiency for the analytical method within the chemical matrix of the sample. The data-quality objective for acceptable spike recovery of trace elements in water samples was a maximum deviation of 25 percent from a theoretical 100-percent recovery of added constituent. At the laboratory, a spiked deionized-water blank and a spiked aliquot of a stream sample were prepared and analyzed along with the original unspiked sample. The differences between the spiked and unspiked sample concentrations were determined and used to compute recovery, in percent, according to equation 3 below:
If the spike recovery for a trace element was outside a range of 75 to 125 percent, the instrument was recalibrated and the entire sample set and spiked samples were reanalyzed for that particular trace element until recoveries were improved to the extent possible. Results of recovery efficiency for individual trace elements in spiked deionized-water blanks and spiked stream samples are presented in tables 11 and 12, respectively. The mean spike recovery for deionized-water samples spiked with trace elements (table 11) ranged from 87.9 to 101.6 percent. The mean spike recovery for spiked stream samples (table 12) ranged from 91.6 to 108.6 percent. The 95 -percent confidence intervals (Taylor, 1987) for the mean of spike recovery for each constituent analyzed in stream samples (table 12) did not exceed a 25-percent deviation from an expected 100-percent recovery.

High or low bias is indicated if the confidence interval does not include 100 percent recovery. All laboratory-spiked deionized-water blank samples (table 11) had confidence intervals for percent recovery that included 100 percent, except filtered iron (87.4-98.8) and unfiltered recoverable lead (93.998.1) percent. All laboratory-spiked stream samples (table 12) had confidence intervals for percent recovery that included 100 percent, except unfiltered recoverable copper (89.7-93.5), unfiltered recoverable lead (93.7-99.9), and unfiltered recoverable zinc (87.5-98.5) percent. Because the mean spike recoveries for all constituents met data-quality objectives, no adjustments were made to analytical results for stream samples on the basis of spike recoveries.

Analytical results for field blanks are presented in table 13. A field blank with constituent concentrations equal to or less than the LRL for the analytical method indicates that the entire process of sample collection, field processing, and laboratory analysis is presumably free of significant contamination. If detectable concentrations in field blanks were equal to or greater than twice the LRL (typical measurement precision at the analytical detection level), the concentrations were noted during data review. Analytical results from the field blank for the next sample set were evaluated for a consistent trend that may indicate systematic contamination. Sporadic, infrequent exceedances of twice the LRL probably represent random contamination or instrument calibration error that is not persistent in the process and which is not likely to cause significant positive bias in a long-term record of analytical results. However, if concentrations for a particular constituent exceeded twice the LRL in field blanks from two consecutive field trips, blank samples were collected from individual components of the processing sequence and were submitted for analysis in order to identify the source of contamination.

Trace-element concentrations in field blanks were almost always less than the LRL. Minor detections equal to the LRL were noted for filtered copper and unfiltered recoverable zinc.

$$
\text { Spike recovery, in percent }=\frac{\text { spiked sample concentration }- \text { unspiked sample concentration }}{\text { spike concentration }} \times 100
$$


No adjustments were made to water-quality sample results based on these minor detections because neither constituent exceeded the LRL in consecutive blank samples.

One major exceedance occurred for filtered cadmium $(0.34 \mu \mathrm{g} / \mathrm{L})$ following an annual equipment blank collected through a D-74 TM sampler. This sample was collected about 3 weeks prior to the associated sampling run, and the collected bottles remained in the Montana District office until they could be submitted with the environmental samples. Also, all subsequent blank samples collected through this sampler had filtered cadmium concentration below the LRL, and all environmental samples collected immediately following this blank had filtered cadmium levels of $0.04 \mu \mathrm{g} / \mathrm{L}$ or less. This random exceedance is not traceable to any systematic processing source, and concentrations in environmental samples apparently were not affected; therefore, no adjustments to environmental data were warranted.

A minor, but more persistent level of potential bias occurred for filtered zinc in blank samples collected from February through the first sampling trip in June, with filtered zinc concentrations ranging from 2 to $4 \mu \mathrm{g} / \mathrm{L}$. In response to this trend, an equipment blank was prepared along with incremental blank samples collected after each step in the processing sequence. Analytical results from this qualityassurance assessment indicated that a small amount of contamination was being introduced from the tubing used in the filtration process. After the tubing was replaced, no further contamination was noted in blank samples. Filtered zinc concentrations for environmental samples were decreased by 2 $4 \mu \mathrm{g} / \mathrm{L}$ for all samples collected during the affected period based on the magnitude of the filtered zinc concentration in the blank sample collected for that specific sampling trip.

\section{Bed-Sediment Data}

Bed-sediment data consist of analyses of trace-element concentrations in the fine-grained and bulk (fine- plus coarsegrained) fractions of the bed-sediment sample. Bed-sediment samples were collected once-annually during low, stable flow conditions and the same season (typically August) as previous samples to facilitate data comparisons among years.

\section{Methods}

Bed-sediment samples were collected in September 2003 using protocols described by E.V. Axtmann (U.S. Geological Survey, written commun., 1994). Samples were collected from the surfaces of streambed deposits in low-velocity areas near the edge of the stream using an acid-washed polypropylene scoop. Whenever possible, samples were collected from both sides of the stream. Three composite samples of fine-grained bed sediment and one composite sample of bulk bed sediment were collected at each site.
Individual samples of fine-grained bed sediment were collected by scooping material from the surfaces of three to five randomly selected deposits along pool or low-velocity areas. The three to five individual samples were combined to form a single composite sample. This collection process was repeated three times to obtain three composite samples. Each composite sample was wet-sieved onsite through a $0.064-\mathrm{mm}$ nylon-mesh sieve using ambient stream water. The fraction of bed sediment in each composite sample that was finer than $0.064 \mathrm{~mm}$ was transferred to an acid-washed $500-\mathrm{mL}$ polyethylene bottle and transported to the laboratory on ice.

Individual samples of bulk bed sediment also were collected by scooping material from the surfaces of three to five randomly selected deposits. The individual unsieved samples were composited into an acid-washed polyethylene bottle to form a single composite sample, each of which were then transported to the laboratory on ice. Because the streambed at most sampling locations is predominantly gravel and cobble, deposits were selected where gravel and cobble could be excluded from the samples. Generally, bulk bed-sediment samples were composed of particles smaller than about $10 \mathrm{~mm}$ in diameter.

Bed-sediment samples were prepared for analysis at the USGS National Research Program laboratory in Menlo Park, Calif. Fine-grained and bulk bed-sediment samples were ovendried at $60^{\circ} \mathrm{C}$ and ground using an acid-washed ceramic mortar and pestle. Duplicate aliquots of approximately $0.6 \mathrm{~g}$ of sediment from each of the three composite fine-grained bed-sediment samples were digested using a hot, concentrated, nitric acid reflux according to methods described by Luoma and Bryan (1981). Duplicate aliquots were similarly digested from the single composite sample of bulk bed sediment. After a digestion period of up to several weeks, the aliquots were evaporated to dryness on a hot plate. The dry residue was redissolved with $20 \mathrm{~mL}$ of $0.6 \mathrm{~N}$ (normal) hydrochloric acid. The reconstituted aliquots then were filtered through a $0.45-\mu \mathrm{m}$ filter using a syringe and in-line disposable filter cartridge. The filtrate was subsequently diluted to a $2: 10,3: 10$, or $5: 10$ ratio with $0.6 \mathrm{~N}$ hydrochloric acid. These final solutions were analyzed for arsenic, cadmium, copper, iron, lead, manganese, nickel, silver, and zinc using inductively coupled argon plasma emission spectroscopy (ICAPES). Chromium data for the 2003 samples were omitted from this report because the values were not reliable.

\section{Results}

Concentrations of trace elements measured in samples of fine-grained and bulk bed sediment collected during September 2003 are summarized in tables 14 and 15, respectively. Liquidphase concentrations, in $\mu \mathrm{g} / \mathrm{mL}$, that were analyzed in the reconstituted aliquots of digested bed sediment were converted to solid-phase concentrations, in $\mu \mathrm{g} / \mathrm{g}$, using the following equation: 


$$
\mu \mathrm{g} / \mathrm{g}=\frac{\mu \mathrm{g} / \mathrm{mL} \times \text { volume of digested sample, in } \mathrm{mL}}{\text { dry weight of sample, in } \mathrm{g} \times \text { dilution ratio }}
$$

The reported solid-phase concentrations in tables 14 and 15 are the means of all analyses of duplicate aliquots from each composite sample collected at the site. Because the conversion from liquid-phase to solid-phase concentration is dependent on both the dilution ratio and the dry weight of the sample, analytical detection limits for some trace elements might differ among stations and among years.

\section{Quality Assurance}

The protocols for field collection and processing of bedsediment samples are designed to prevent contamination from metal sources. Non-metallic sampling and processing equipment was acid-washed and rinsed with deionized water prior to the first sample collection. Nylon-mesh sieves were washed in a laboratory-grade detergent and rinsed with deionized water. All equipment was given a final rinse onsite with stream water. Sampling equipment that was reused at each site was rinsed between sites with 10 -percent nitric acid, deionized water, and stream water. Separate sieves were used at each site and, therefore, did not require between-site cleaning.

Quality assurance of analytical results for bed sediment included laboratory instrument calibration with standard solutions and analysis of quality-control samples designed to identify the presence and magnitude of bias (E.V. Axtmann, U.S. Geological Survey, written commun., 1994). Quality-control samples consisted of standard reference materials and procedural blanks. Each type of sample was analyzed in a proportion equivalent to about 10 to 20 percent of the total number of bedsediment samples.

Standard reference materials (SRMs) are commercially prepared materials that have certified concentrations of trace elements. Replicate analyses of SRMs are used to indicate the precision (reproducibility) of analytical results and the ability of the method to accurately measure a known quantity of a constituent. Recovery efficiency of trace-element analyses of SRMs for bed sediment is summarized in table 16. Two SRMs consisting of agricultural soils representing low and high concentrations of trace elements were analyzed to test recovery efficiency for a range of concentrations generally similar to those occurring in the upper Clark Fork basin. The digestion process used to analyze bed-sediment samples is not a "total" digestion (does not liberate elements associated with crystalline lattices); therefore, 100-percent recovery may not be achieved for elements strongly bound to the sediment. The percent recovery of trace elements in SRM analyses that use less than a total digestion is useful to indicate which trace elements display strong sediment-binding characteristics in the SRM and whether analytical recovery is consistent between multiple sets of analyses.

Although data-quality objectives have not been established for bed sediment, percent recoveries shown in table 16 illustrate analytical performance. Cadmium and silver concen- trations were less than the analytical detection limit in the lowconcentration standard (SRM 2709). The reason for the lack of measurable recoveries for cadmium and silver in this SRM is believed to be the result of analyzing concentrations very close to the detection limit $(0.0001 \mu \mathrm{g} / \mathrm{mL}$ and $0.001 \mu \mathrm{g} / \mathrm{mL}$, respectively) coupled with signal enhancement resulting from matrix interference. Percent recoveries for copper were 50.4 percent for the low-concentration standard (SRM 2709) and 66.2 percent for the high-concentration standard (SRM 2711). Arsenic and zinc in SRM 2709 and silver in SRM 2711 also displayed low recoveries $(68.1,76.2$, and 78.5 percent, respectively). Although these recovery values are relatively low, no adjustments were made to the data because the results from instrument calibration with standard solutions were well within acceptable limits (5-10 percent). Mean recoveries were within 20 percent of complete recovery for all other constituents in both the low- and high-concentration SRMs and ranged from about 83 to 116 percent. No adjustments were made to traceelement concentrations in bed-sediment samples on the basis of recovery efficiencies.

Procedural blanks for bed-sediment samples consisted of the same reagents used for sample digestion and reconstitution. Concentrated nitric acid used for sample digestion was heated and evaporated to dryness. After evaporation, $0.6 \mathrm{~N}$ hydrochloric acid was added to the dry residue. Procedural blanks, therefore, represent the same chemical matrix as the reagents used to digest and reconstitute bed-sediment samples. No dilution of the reagents was made prior to analysis in order to maximize and detect any potential contamination associated with sample handling and analysis in the laboratory environment. Results of trace-element analyses of procedural blanks for bed sediment are in table 17.

Analytical results of procedural blanks are reported as a liquid-phase concentration, in $\mu \mathrm{g} / \mathrm{mL}$, which is equivalent to parts per million. Determination of the significance of a detectable blank concentration is based on the magnitude of the equivalent solid-phase concentration, in $\mu \mathrm{g} / \mathrm{g}$, relative to the ambient concentration of the trace element in bed-sediment samples. If a detectable blank concentration represents 10 percent or more of the ambient solid-phase concentration, then the blank concentration is subtracted to remove potential contamination bias. One procedural blank had a cadmium concentration at the analytical detection limit. However, because this one sample had a concentration less than 1 percent of the ambient concentration in environmental samples, no adjustments were made to traceelement concentrations in bed-sediment samples on the basis of procedural blanks.

\section{Biological Data}

Biological data consist of analyses of trace-element concentrations in the whole-body tissue of aquatic benthic insects. Insect samples were collected once-annually at the same sites and dates as bed-sediment samples (table 1), allowing for a 
direct comparison of biological data among years and with bedsediment data.

\section{Methods}

Insect samples were collected using protocols described in Hornberger and others (1997). Immature stages of benthic insects were collected using a large nylon-mesh kick net. A single riffle at each station was sampled repeatedly until an adequate number of individuals was collected to provide sufficient mass for analysis. Targeted taxa for collection were Hydropsyche spp. and Arctopsyche grandis of the Order Trichoptera (caddisflies), and Claassenia sabulosa of the Order Plecoptera (stoneflies). Samples of each taxon were sorted by genus and placed in acid-washed plastic containers. Samples were frozen on dry ice within 30 minutes of collection in a small amount of ambient river water. In previous years (1986-98), benthic insects were depurated for a period of 6-8 hours in an effort to evacuate gut contents. In 1998, a comparison of samples collected using both methods showed no significant difference in metal concentrations in benthic insects, with the exception of copper. Average copper concentrations in depurated samples were 8-25 percent lower than in samples frozen within $30 \mathrm{~min}-$ utes of collection (M.I. Hornberger, unpub. data, 2000). The change in the field protocol minimizes the chance of metal loss through cell membranes during depuration and is consistent with methods established by Cain and Luoma (1998). However, caution needs to be exercised in comparing recent copper data for insects with earlier data because of the possibility of higher concentrations resulting from the change in field protocol.

Insect samples were processed and analyzed at the USGS National Research Program laboratory in Menlo Park, Calif. Insects were thawed and rinsed with ultra-pure deionized water to remove particulate matter and then sorted to their lowest possible taxonomic level. If large numbers of specimens had been collected at a station, similar-sized individuals were composited into replicate subsamples. Subsamples were placed in tared scintillation vials and oven-dried at $70^{\circ} \mathrm{C}$. Subsamples were weighed to obtain a final dry weight and digested by reflux using concentrated nitric acid (Cain and others, 1992). After digestion, insect samples were evaporated to dryness on a hot plate. The dry residue was reconstituted in $0.6 \mathrm{~N}$ hydrochloric acid, filtered through a $0.45-\mu \mathrm{m}$ filter, and analyzed undiluted by ICAPES for arsenic, cadmium, chromium, copper, iron, lead, manganese, nickel, and zinc.

\section{Results}

Concentrations of trace elements in whole-body tissue of aquatic insects collected during September 2003 are summarized in table 18. The variability in the number of composite samples among species and among sites reflects differences in insect abundance, with the number of composite samples increasing with the relative abundance of insects. Liquid-phase concentrations analyzed in the reconstituted samples were converted to solid-phase concentrations using equation 4 . As with bed sediment, minimum reporting levels may differ among sites as a result of variable sample weights. In general, the smaller the biological sample weight (a function of insect abundance), the higher the minimum reporting level. Therefore, higher minimum reporting levels do not necessarily imply a higher traceelement concentration in tissue.

Two species of Hydropsyche were targeted for collection in this study due to their occurrence at most, but not all, sites: Hydropsyche cockerelli and Hydropsyche occidentalis. Hydropsyche species that could not be positively identified were considered to belong to the morosa group and are categorized as Hydropsyche spp. or Hydropsyche morosa group (in previous reports). Arctopsyche grandis and Claasenia sabulosa also were collected, where available, to represent additional insect taxa that are fairly widely distributed in the upper Clark Fork basin.

\section{Quality Assurance}

The protocols for field collection and processing of biota samples are designed to prevent contamination from metal sources. Non-metallic nets, sampling equipment, and processing equipment were employed in all sample collection. Equipment was acid-washed and rinsed in ultra-pure deionized water prior to the first sample collection. Nets and equipment were thoroughly rinsed in ambient stream water at each new mainstem station. New nets were used for all tributary stations. Biota samples were collected sequentially at stations along an increasing concentration gradient to minimize effects from potential station-to-station carryover contamination.

Quality assurance of analytical results for biota samples included laboratory instrument calibration with standard solutions and analyses of quality-control samples designed to quantify precision and to identify the presence and magnitude of bias. Quality-control samples consisted of a SRM and procedural blanks. Each type of sample was analyzed in a proportion equivalent to about 10 to 20 percent of the total number of biota samples.

Recovery efficiency for trace-element analyses of the SRM for biota is summarized in table 19. The reference material tested was lobster hepatopancreas. Data-quality objectives have not been established for analytical recovery in biota, but percent recoveries are shown to illustrate analytical performance. Mean SRM recoveries were within 12 percent of certified values for arsenic, cadmium, copper, iron, manganese, and zinc. Mean recoveries for chromium and nickel were within 26 percent, and recoveries for lead were within 52 percent. The low recoveries for lead were attributed to 7 of the 12 samples having concentrations less than the instrument analytical detection limit. Additionally, a quality-control standard with a similar solution concentration as the SRM samples was analyzed throughout the analysis. Recoveries for all reported elements were within 10 percent of the quality-control standard. 


\section{in the Upper Clark Fork Basin, Montana}

Results of trace-element analyses of procedural blanks for biota are in table 20. Procedural blanks for biota consisted of the same reagents used to digest and reconstitute tissue of aquatic insects. The blanks were analyzed undiluted at a proportion of one blank per site. Analytical results for all blanks were less than the analytical detection limit; thus, no contamination bias was indicated.

\section{Statistical Summaries of Data}

Statistical summaries of water-quality, bed-sediment, and biological data are provided in tables 21-24 for the period of record at each station since 1985. The summaries include the period of record, number of samples, maximum, minimum, mean, and median of concentrations.

Statistical summaries of water-quality data (table 21) are based on results of cross-sectional samples collected periodically by the USGS during the period of record for each station. The summaries do not include data for supplemental single-vertical samples collected during several years by a contract observer at Clark Fork at Turah Bridge, near Bonner; Blackfoot River near Bonner; and Clark Fork above Missoula. Inclusion of results for supplemental samples that targeted high-flow conditions or maintenance drawdowns of Milltown Reservoir would disproportionately skew the long-term statistics at these three sites relative to the other sites in the network. Statistical summaries of bed-sediment (table 22 and 23) and biological data (table 24) are based on results of samples collected onceannually during the indicated years. Because not all stations were sampled for bed sediment and biota every year, the data for some sites do not represent a consecutive annual record.

Sample sizes and statistics for bed-sediment data are based on a compilation of single annual-mean concentrations determined from the combined results of multiple composite samples for a given year. Therefore, sample sizes for bed sediment represent the number of years sampled. In contrast, sample sizes and statistics for biological data are based on individual analyses for each composite sample collected in an individual year, rather than on a single annual-mean concentration from all composites combined. Biota sample sizes reflect differences in species abundance at each site and among all years. As a result, the statistics for biota describe a wider range of variation in trace-element concentrations than would be evident if results from individual composite samples were averaged. The abundance of aquatic insects at a particular site in a given year limits the biomass of the sample which, in turn, may result in variable analytical detection limits. Where analytical detection limits vary among years, statistical summaries are provided only as a general indication of the range of detection.

The presence or absence of insect species at a given site can vary among years and may result in different taxa being analyzed in the long-term period of record. Because Hydropsyche insects were not sorted to the species level during 1986-89, statistics for stations sampled during those years are based on the results of all Hydropsyche species combined. At some sites, statistics for the Hydropsyche morosa group are based on the combined results for two or more species because these samples could not be identified clearly to the species level, but the individual insects had morosa characteristics.

\section{References Cited}

Axtmann, E.V., Cain, D.J., and Luoma, S.N., 1997, Effect of tributary inflows on the distribution of trace metals in finegrained sediment and benthic insects of the Clark Fork River, Montana: Environmental Science and Technology, v. 31, no. 3, p. 750-758.

Axtmann, E.V., and Luoma, S.N., 1991, Large scale distribution of metal contamination in the fine-grained sediment of the Clark Fork River, Montana: Applied Geochemistry, v. 6, no. 6, p. 75-88.

Cain, D.J., and Luoma, S.N., 1998, Metal exposures to native populations of the caddisfly Hydropsyche (TrichopteraHydropsychidae) determined from cytosolic and whole body metal concentrations: Hydrobiologia, v. 386, p. 103-117.

Cain, D.J., Luoma, S.N., and Axtmann, E.V., 1995, Influence of gut content in immature aquatic insects on assessments of environmental metal contamination: Canadian Journal of Fisheries and Aquatic Sciences, v. 52, no. 12, p. 2736-2746.

Cain, D.J., Luoma, S.N., Carter, J.L., and Ferd, S.V., 1992, Aquatic insects as bioindicators of trace element contamination in cobble-bottom rivers and streams: Canadian Journal of Fisheries and Aquatic Sciences, v. 49, no. 10, p. 21412154.

Childress, C.T., Foreman, W.T., Connor, B.F., and Maloney, T.J., 1999, New reporting procedures based on long-term method detection levels and some considerations for interpretations of water-quality data provided by the U.S. Geological Survey National Water Quality Laboratory: U.S. Geological Survey Open-File Report 99-193, 19 p.

Dodge, K.A., Hornberger, M.I., and Axtmann, E.V., 1996, Water-quality, bed-sediment, and biological data (October 1994 through September 1995) and statistical summaries of data for streams in the upper Clark Fork basin, Montana: U.S. Geological Survey Open-File Report 96-432, 109 p.

Dodge, K.A., Hornberger, M.I., and Axtmann, E.V., 1997, Water-quality, bed-sediment, and biological data (October 1995 through September 1996) and statistical summaries of data for streams in the upper Clark Fork basin, Montana: U.S. Geological Survey Open-File Report 97-552, 91 p.

Dodge, K.A., Hornberger, M.I., and Axtmann, E.V., 1998, Water-quality, bed-sediment, and biological data (October 1996 through September 1997) and statistical summaries of data for streams in the upper Clark Fork basin, Montana: U.S. Geological Survey Open-File Report 98-407, 102 p.

Dodge, K.A., Hornberger, M.I., and Bouse, R.M., 1999, Waterquality, bed-sediment, and biological data (October 1997 through September 1998) and statistical summaries of data 
for streams in the upper Clark Fork basin, Montana: U.S. Geological Survey Open-File Report 99-251, 102 p.

Dodge, K.A., Hornberger, M.I., and David, C.P.C., 2000, Water-quality, bed-sediment, and biological data (October 1998 through September 1999) and statistical summaries of data for streams in the upper Clark Fork basin, Montana: U.S. Geological Survey Open-File Report 00-370, 102 p.

Dodge, K.A., Hornberger, M.I., and David, C.P.C., 2001, Water-quality, bed-sediment, and biological data (October 1999 through September 2000) and statistical summaries of data for streams in the upper Clark Fork basin, Montana: U.S. Geological Survey Open-File Report 01-379, 95 p.

Dodge, K.A., Hornberger, M.I., and David, C.P.C., 2002 , Water-quality, bed-sediment, and biological data (October 2000 through September 2001) and statistical summaries of data for streams in the upper Clark Fork basin, Montana: U.S. Geological Survey Open-File Report 02-358, 94 p.

Dodge, K.A., Hornberger, M.I., and Lavigne, I.R., 2003, Waterquality, bed-sediment, and biological data (October 2001 through September 2002) and statistical summaries of data for streams in the upper Clark Fork basin, Montana: U.S. Geological Survey Open-File Report 03-356, 95 p.

Edwards, T.K., and Glysson, G.D., 1999, Field methods for measurement of fluvial sediment: U.S. Geological Survey Techniques of Water-Resources Investigations, book 3, chap. C2, 89 p.

Faires, L.M., 1993, Methods of analysis by the U.S. Geological Survey National Water Quality Laboratory - Determination of metals in water by inductively coupled plasma-mass spectrometry: U.S. Geological Survey Open-File Report 92-634, $28 \mathrm{p}$.

Fishman, M.J., ed., 1993, Methods of analysis by the U.S. Geological Survey National Water Quality Laboratory - Determination of inorganic and organic constituents in water and fluvial sediments: U.S. Geological Survey Open-File Report 93-125, 217 p.

Friedman, L.C., and Erdmann, D.E., 1982, Quality assurance practices for the chemical and biological analyses of water and fluvial sediments: U.S. Geological Survey Techniques of Water-Resources Investigations, book 5, chap. A6, $181 \mathrm{p}$.

Garbarino, J.R., 1999, Methods of analysis by the U.S. Geological Survey National Water Quality Laboratory - Determination of dissolved arsenic, boron, lithium, selenium, strontium, thallium, and vanadium using inductively coupled plasma-mass spectrometry: U.S. Geological Survey OpenFile Report 99-093, 31 p.

Garbarino, J.R., and Struzeski, T.M., 1998, Methods of analysis by the U.S. Geological Survey National Water Quality Laboratory - Determination of elements in whole-water digests using inductively coupled plasma-optical emission spectrometry and inductively coupled plasma-mass spectrometry: U.S. Geological Survey Open-File Report 98-165, 101 p.

Guy, H.P., 1969, Laboratory theory and methods for sediment analysis: U.S. Geological Survey Techniques of WaterResources Investigations, book 5, chap. C1, 58 p.
Helsel, D.R., and Cohn, T.A., 1988, Estimation of descriptive statistics for multiply censored water quality data: Water Resources Research, v. 24, no. 12, p. 1997-2004.

Hoffman, G.L., Fishman, M.J., and Garbarino, J.R., 1996, Methods of analysis by the U.S. Geological Survey National Water Quality Laboratory - In-bottle acid digestion of whole-water samples: U.S. Geological Survey Open-File Report 96-225, 28 p.

Hornberger, M.I., Lambing, J.H., Luoma, S.N., and Axtmann, E.V., 1997, Spatial and temporal trends of trace metals in surface water, bed sediment, and biota of the upper Clark Fork basin, Montana, 1985-95: U.S. Geological Survey Open-File Report 97-669, 84 p.

Horowitz, A.J., Demas, C.R., Fitzgerald, K.K., Miller, T.L., and Rickert, D.A., 1994, U.S. Geological Survey protocol for the collection and processing of surface-water samples for the subsequent determination of inorganic constituents in filtered water: U.S. Geological Survey Open-File Report 94-539, $57 \mathrm{p}$.

Jones, B.E., 1987, Quality control manual of the U.S. Geological Survey's National Water Quality Laboratory: U.S. Geological Survey Open-File Report 87-457, 17 p.

Jones, S.R., and Garbarino, J.R., 1999, Methods of analysis by the U.S. Geological Survey National Water Quality Laboratory - Determination of arsenic and selenium in water and sediment by graphite furnace-atomic absorption spectrometry: U.S. Geological Survey Open-File Report 98-639, 39 p.

Knapton, J.R., and Nimick, D.A., 1991, Quality assurance for water-quality activities of the U.S. Geological Survey in Montana: U.S. Geological Survey Open-File Report 91-216, $41 \mathrm{p}$.

Lambing, J.H., 1987, Water-quality data for the Clark Fork and selected tributaries from Deer Lodge to Milltown, Montana, March 1985 through June 1986: U.S. Geological Survey Open-File Report 87-110, 48 p.

Lambing, J.H., 1988, Water-quality data (July 1986 through September 1987) and statistical summaries (March 1985 through September 1987) for the Clark Fork and selected tributaries from Deer Lodge to Missoula, Montana: U.S. Geological Survey Open-File Report 88-308, 55 p.

Lambing, J.H., 1989, Water-quality data (October 1987 through September 1988) and statistical summaries (March 1985 through September 1988) for the Clark Fork and selected tributaries from Galen to Missoula, Montana: U.S. Geological Survey Open-File Report 89-229, 51 p.

Lambing, J.H., 1990, Water-quality data (October 1988 through September 1989) and statistical summaries (March 1985 through September 1989) for the Clark Fork and selected tributaries from Galen to Missoula, Montana: U.S. Geological Survey Open-File Report 90-168, 68 p.

Lambing, J.H., 1991, Water-quality and transport characteristics of suspended sediment and trace elements in streamflow of the upper Clark Fork basin from Galen to Missoula, Montana, 1985-90: U.S. Geological Survey Water-Resources Investigations Report 91-4139, 73 p. 
14 Water-Quality, Bed-Sediment, and Biological Data (October 2002 through September 2003) and Statistical Summaries of Data for Streams in the Upper Clark Fork Basin, Montana

Lambing, J.H., and Dodge, K.A., 1993, Quality assurance for laboratory analysis of suspended-sediment samples by the U.S. Geological Survey in Montana: U.S. Geological Survey Open-File Report 93-131, 34 p.

Lambing, J.H., Hornberger, M.I., Axtmann, E.V., and Dodge, K.A., 1995, Water-quality, bed-sediment, and biological data (October 1993 through September 1994) and statistical summaries of data for streams in the upper Clark Fork basin, Montana: U.S. Geological Survey Open-File Report 95-429, $104 \mathrm{p}$.

Lambing, J.H., Hornberger, M.I., Axtmann, E.V., and Pope, D.A., 1994, Water-quality, bed-sediment, and biological data (October 1992 through September 1993) and statistical summaries of water-quality data (March 1985 through September 1993) for streams in the upper Clark Fork basin, Montana:

U.S. Geological Survey Open-File Report 94-375, 85 p.

Luoma, S.N., and Bryan, G.W., 1981, A statistical assessment of the form of trace metals in oxidized estuarine sediments employing chemical extractants: Science of the Total Environment, v. 17, no. 17, p. 167-196.

Porterfield, George, 1972, Computation of fluvial-sediment discharge: U.S. Geological Survey Techniques of WaterResources Investigations, book 3, chap. C3, 66 p.
Pritt, J.W., and Raese, J.W., eds., 1995, Quality assurance/quality control manual-National Water Quality Laboratory: U.S. Geological Survey Open-File Report 95443, 35 p.

Rantz, S.E., and others, 1982, Measurement and computation of streamflow: U.S. Geological Survey Water-Supply Paper 2175,2 v., 631 p.

Taylor, J.K., 1987, Quality assurance of chemical measurements: Chelsea, Mich., Lewis Publishers, 328 p.

Timme, P.J., 1994, National Water Quality Laboratory 1994 services catalog: U.S. Geological Survey Open-File Report 94-304, $103 \mathrm{p}$.

U.S. Geological Survey, variously dated, National field manual for the collection of water-quality data: U.S. Geological Survey Techniques of Water-Resources Investigations, book 9, chaps. A1-A-9; available online at http://pubs.water.usgs.gov/twri9A

Ward, J.R., and Harr, C.A., eds., 1990, Methods for collection and processing of surface-water and bed-material samples for physical and chemical analyses: U.S. Geological Survey Open-File Report 90-140, 71 p. 
DATA 15

\section{DATA}


Table 4. Water-quality data for the upper Clark Fork basin, Montana, October 2002 through September 2003

[The terms "filtered" and "unfiltered recoverable" replace the former terms "dissolved" and "total recoverable," respectively. Abbreviations: $\mathrm{ft}^{3} / \mathrm{s}$, cubic feet per second; ${ }^{\circ} \mathrm{C}$, degrees Celsius; E, estimated; $\mu \mathrm{g} / \mathrm{L}$, micrograms per liter; $\mu \mathrm{S} / \mathrm{cm}$, microsiemens per centimeter at $25^{\circ} \mathrm{C} ; \mathrm{mg} / \mathrm{L}$,

milligrams per liter; mm, millimeter; ton/d, tons per day. Symbols: <, less than analytical detection limit; --, no data]

12323230--BLACKTAIL CREEK AT HARRISON AVENUE, AT BUTTE, MONT.

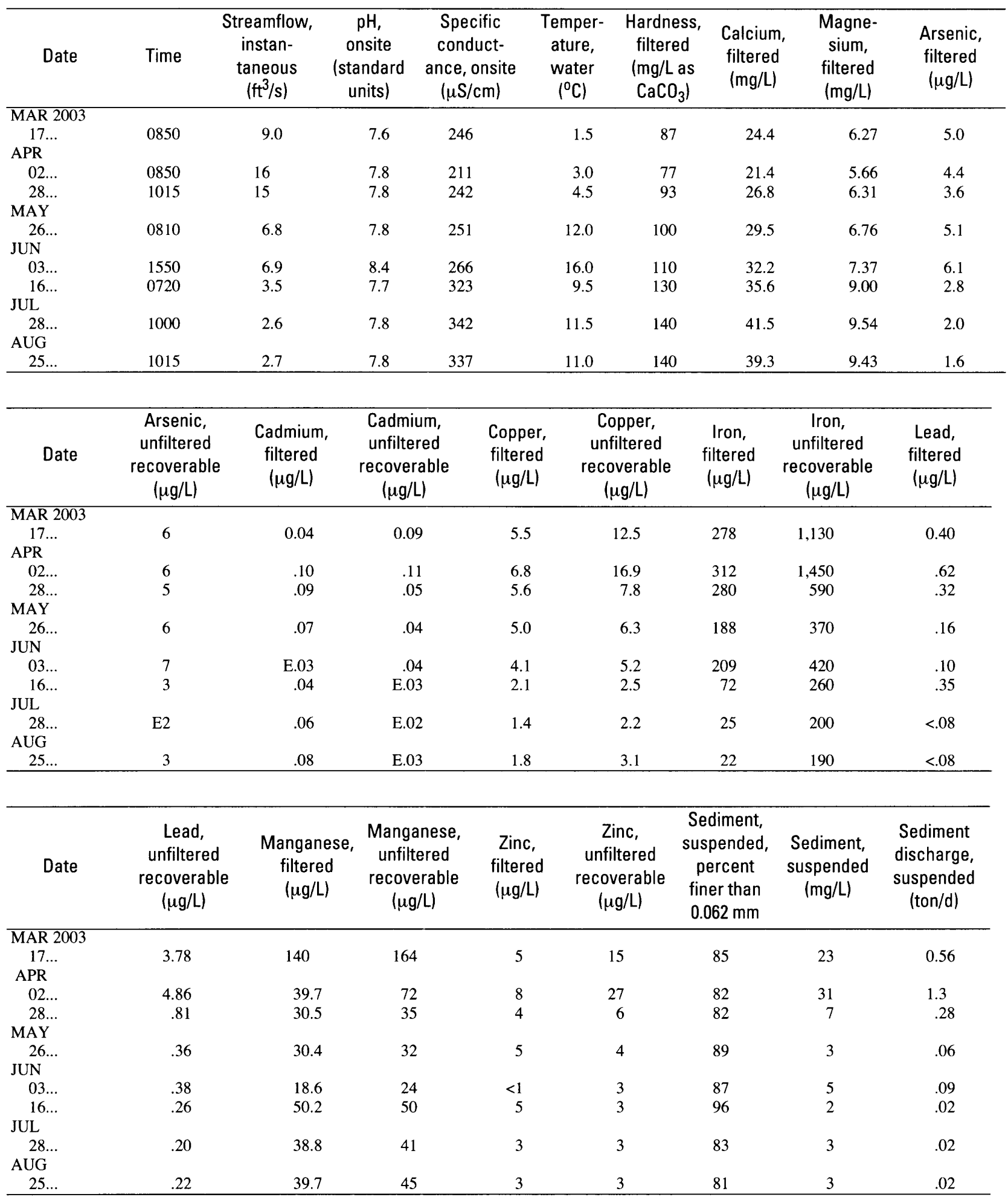


Table 4. Water-quality data for the upper Clark Fork basin, Montana, October 2002 through September 2003-Continued 12323250--SILVER BOW CREEK BELOW BLACKTAIL CREEK, AT BUTTE, MONT.

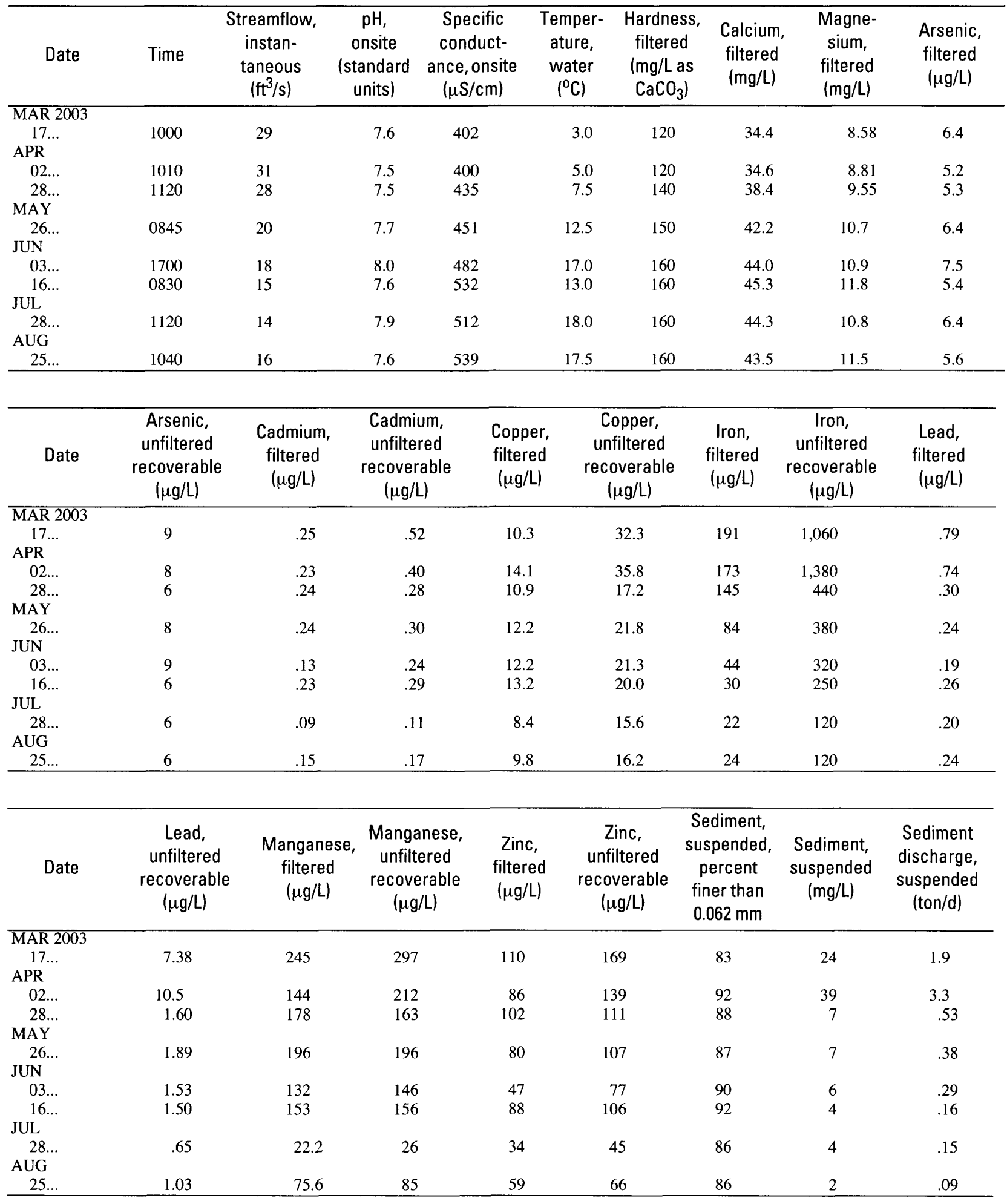


18 Water-Quality, Bed-Sediment, and Biological Data (October 2002 through September 2003) and Statistical Summaries of Data for Streams in the Upper Clark Fork Basin, Montana

Table 4. Water-quality data for the upper Clark Fork basin, Montana, October 2002 through September 2003-Continued

12323600--SILVER BOW CREEK AT OPPORTUNITY, MONT.

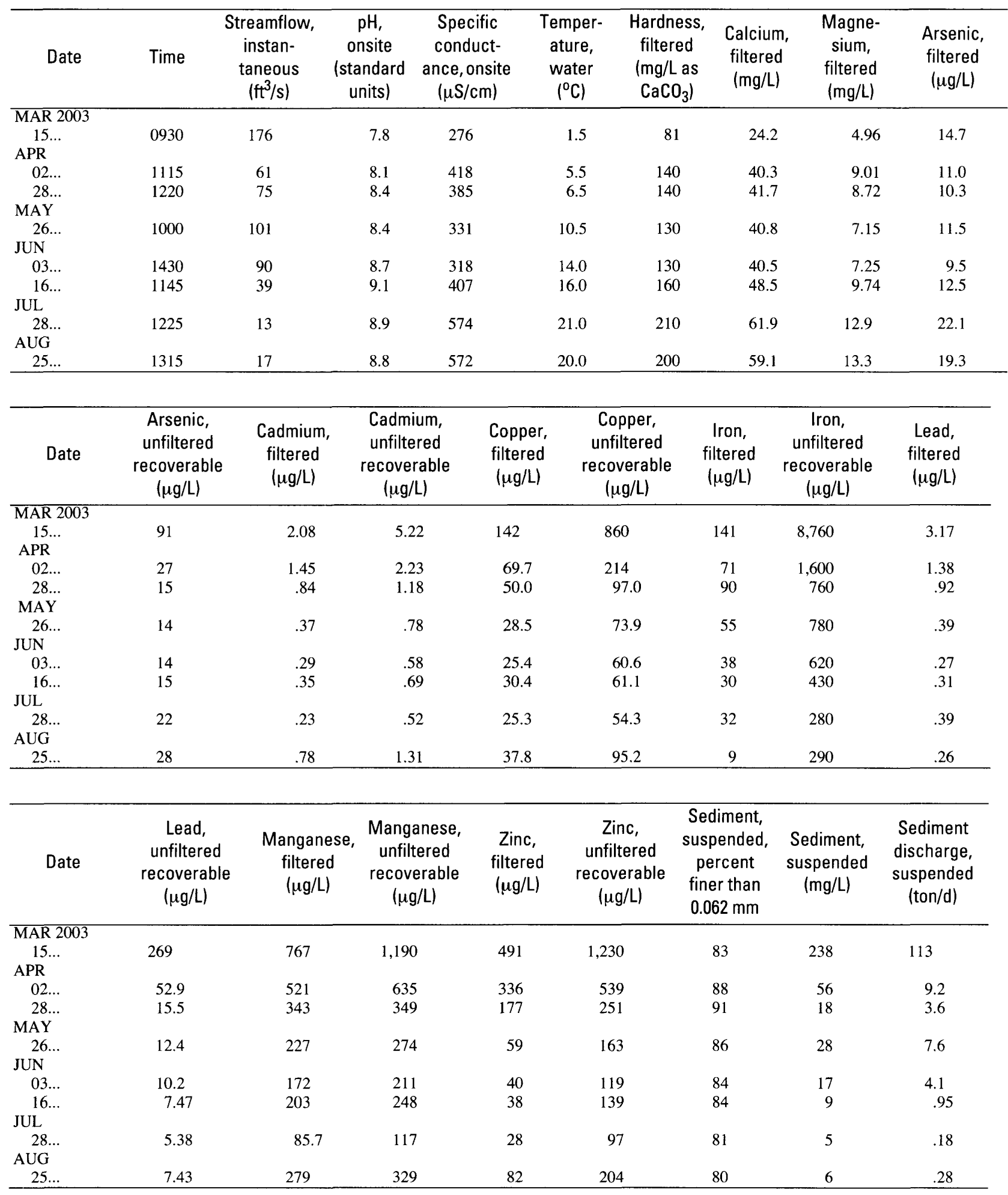


Table 4. Water-quality data for the upper Clark Fork basin, Montana, October 2002 through September 2003-Continued 12323700--MILL CREEK AT OPPORTUNITY, MONT.

\begin{tabular}{|c|c|c|c|c|c|c|c|c|c|c|}
\hline Date & Time & $\begin{array}{l}\text { Streamflow, } \\
\text { instan- } \\
\text { taneous } \\
\left(\mathrm{ft}^{3} / \mathrm{s}\right)\end{array}$ & $\begin{array}{c}\mathrm{pH}, \\
\text { onsite } \\
\text { (standard } \\
\text { units) }\end{array}$ & $\begin{array}{r}\text { Spec } \\
\text { cond } \\
\text { ance, } 0 \\
(\mu S / c\end{array}$ & $\begin{array}{l}\text { fic } \\
\text { ct- } \\
\text { site } \\
\text { m) } \\
\end{array}$ & $\begin{array}{l}\text { Temper- } \\
\text { ature, } \\
\text { water } \\
\left({ }^{\circ} \mathrm{C}\right)\end{array}$ & $\begin{array}{r}\text { Hardnes } \\
\text { filtered } \\
(\mathrm{mg} / \mathrm{L} \mathrm{a} \\
\left.\mathrm{CaCO}_{3}\right)\end{array}$ & $\begin{array}{c}\text { Calcium, } \\
\text { filtered } \\
(\mathrm{mg} / \mathrm{L})\end{array}$ & $\begin{array}{l}\text { Magne- } \\
\text { sium, } \\
\text { filtered } \\
\text { (mg/L) }\end{array}$ & $\begin{array}{c}\text { Arsenic, } \\
\text { filtered } \\
(\mu \mathrm{g} / \mathrm{L})\end{array}$ \\
\hline \multicolumn{11}{|l|}{ MAR 2003} \\
\hline $\begin{array}{l}17 \ldots \\
\text { APR }\end{array}$ & 1210 & 9.6 & 8.2 & 207 & & 3.0 & 85 & 23.0 & 6.57 & 37.0 \\
\hline $02 \ldots$ & 1255 & 14 & 8.0 & 186 & & 5.0 & 73 & 20.0 & 5.69 & 33.2 \\
\hline $28 \ldots$ & 1345 & 21 & 8.0 & 129 & & 5.5 & 51 & 14.4 & 3.74 & 33.6 \\
\hline \multicolumn{11}{|l|}{ MAY } \\
\hline \multicolumn{11}{|l|}{ JUN } \\
\hline $11 \ldots$ & 1430 & 261 & 7.8 & 59 & & 9.5 & 24 & 7.01 & 1.57 & 23.0 \\
\hline $16 \ldots$ & 1005 & 79 & 7.9 & 83 & & 9.0 & 37 & 10.6 & 2.55 & 20.5 \\
\hline \multicolumn{11}{|l|}{ JUL } \\
\hline \multicolumn{11}{|l|}{ AUG } \\
\hline $25 \ldots$ & 1230 & 2.1 & 8.1 & 195 & & 16.0 & 85 & 23.5 & 6.32 & 29.7 \\
\hline Date & $\begin{array}{c}\text { Arsenic, } \\
\text { unfiltered } \\
\text { recoverable } \\
(\mu \mathrm{g} / \mathrm{L})\end{array}$ & $\begin{array}{c}\text { Cadmium, } \\
\text { filtered } \\
(\mu \mathrm{g} / \mathrm{L})\end{array}$ & $\begin{array}{r}\text { Cadm } \\
\text { unfilt } \\
\text { recove } \\
(\mu \mathrm{g}\end{array}$ & $\begin{array}{l}\text { ium, } \\
\text { red } \\
\text { rable } \\
\text { L) }\end{array}$ & $\begin{array}{c}\text { Copper, } \\
\text { filtered } \\
(\mu \mathrm{g} / \mathrm{L})\end{array}$ & & $\begin{array}{l}\text { Copper, } \\
\text { nfiltered } \\
\text { coverable } \\
(\mu \mathrm{g} / \mathrm{L})\end{array}$ & $\begin{array}{l}\text { Iron, } \\
\text { filtered } \\
(\mu \mathrm{g} / \mathrm{L})\end{array}$ & $\begin{array}{c}\text { Iron, } \\
\text { unfiltered } \\
\text { recoverable } \\
(\mu \mathrm{g} / \mathrm{L})\end{array}$ & $\begin{array}{c}\text { Lead, } \\
\text { filtered } \\
(\mu \mathrm{g} / \mathrm{L})\end{array}$ \\
\hline \multicolumn{11}{|l|}{ MAR $200 \overline{3}$} \\
\hline $\begin{array}{l}17 \ldots \\
\text { APR }\end{array}$ & 38 & .08 & & 1 & 3.7 & & 5.0 & 39 & 120 & .18 \\
\hline $02 \ldots$ & 36 & .09 & & 11 & 4.7 & & 7.5 & 37 & 200 & 17 \\
\hline $28 \ldots$ & 35 & .10 & & 15 & 5.2 & & 8.0 & 51 & 210 & .23 \\
\hline \multicolumn{11}{|l|}{ MAY } \\
\hline \multicolumn{11}{|l|}{ JUN } \\
\hline $11 \ldots$ & 36 & .08 & & 51 & 6.0 & & 25.1 & 50 & 1,300 & .27 \\
\hline $16 \ldots$ & 22 & .06 & & 13 & 3.3 & & 6.9 & 41 & 320 & .22 \\
\hline \multicolumn{11}{|l|}{ JUL } \\
\hline $28 \ldots$ & 32 & .05 & & 6 & 2.4 & & 3.3 & 45 & 100 & .14 \\
\hline $25 \ldots$ & 35 & .05 & & 06 & 2.2 & & 3.2 & 60 & 100 & .11 \\
\hline Date & $\begin{array}{l}\text { Lead, } \\
\text { unfiltered } \\
\text { recoverable } \\
(\mu \mathrm{g} / \mathrm{L})\end{array}$ & $\begin{array}{c}\text { Manganese, } \\
\text { filtered } \\
(\mu g / L)\end{array}$ & $\begin{array}{r}\text { Mang } \\
\text { unfil } \\
\text { recov } \\
(\mu\end{array}$ & $\begin{array}{l}\text { anese, } \\
\text { tered } \\
\text { erable } \\
\text { g/L) }\end{array}$ & $\begin{array}{c}\text { Zinc, } \\
\text { filtered } \\
(\mu \mathrm{g} / \mathrm{L})\end{array}$ & $\begin{array}{r}2 \\
\text { unt } \\
\text { reco }\end{array}$ & $\begin{array}{l}\text { Zinc, } \\
\text { ofiltered } \\
\text { overable } \\
\text { ( } \mu \mathrm{g} / \mathrm{L})\end{array}$ & $\begin{array}{l}\text { Sediment, } \\
\text { suspended, } \\
\text { percent } \\
\text { finer than } \\
0.062 \mathrm{~mm}\end{array}$ & $\begin{array}{l}\text { Sediment, } \\
\text { suspended } \\
\text { (mg/L) }\end{array}$ & $\begin{array}{c}\text { Sediment } \\
\text { discharge, } \\
\text { suspended } \\
\text { (ton/d) }\end{array}$ \\
\hline \multicolumn{11}{|l|}{ MAR 2003} \\
\hline $17 \ldots$ & .48 & 3.6 & & 6 & 3 & & 5 & 78 & 1 & .03 \\
\hline \multicolumn{11}{|l|}{ APR } \\
\hline $02 \ldots$ & 1.08 & 4.8 & 1 & & 4 & & 8 & 87 & 5 & .19 \\
\hline $28 \ldots$ & 1.19 & 4.4 & 1 & & 6 & & 7 & 78 & 6 & .34 \\
\hline \multicolumn{11}{|l|}{ MAY } \\
\hline \multicolumn{11}{|l|}{ JUN } \\
\hline $11 \ldots$ & 8.01 & 7.0 & 6 & & 4 & & 26 & 52 & 78 & 55 \\
\hline $\begin{array}{l}16 \ldots \\
\text { JUL }\end{array}$ & 1.74 & 6.4 & 1 & & 3 & & 10 & 51 & 10 & 2.1 \\
\hline $\begin{array}{c}28 \ldots \\
\text { AUG }\end{array}$ & .30 & 11.1 & 1 & & 2 & & 2 & 88 & 1 & .01 \\
\hline $25 \ldots$ & .17 & 9.8 & 1 & & 2 & & 3 & 80 & 1 & .01 \\
\hline
\end{tabular}


20 Water-Quality, Bed-Sediment, and Biological Data (October 2002 through September 2003) and Statistical Summaries of Data for Streams in the Upper Clark Fork Basin, Montana

Table 4. Water-quality data for the upper Clark Fork basin, Montana, October 2002 through September 2003-Continued

12323720--WILLOW CREEK AT OPPORTUNITY, MONT.

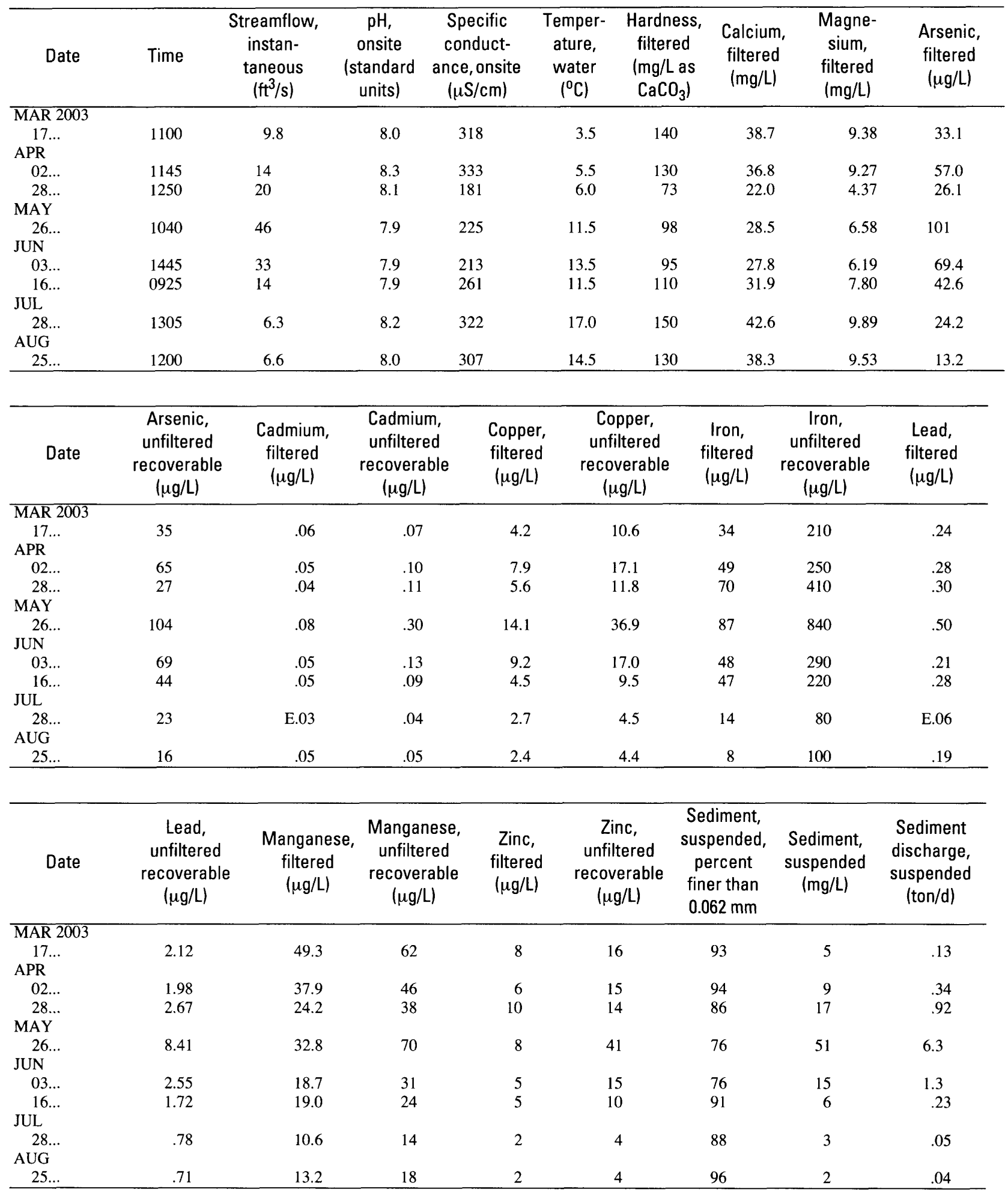


Table 4. Water-quality data for the upper Clark Fork basin, Montana, October 2002 through September 2003-Continued

12323750--SILVER BOW CREEK AT WARM SPRINGS, MONT.

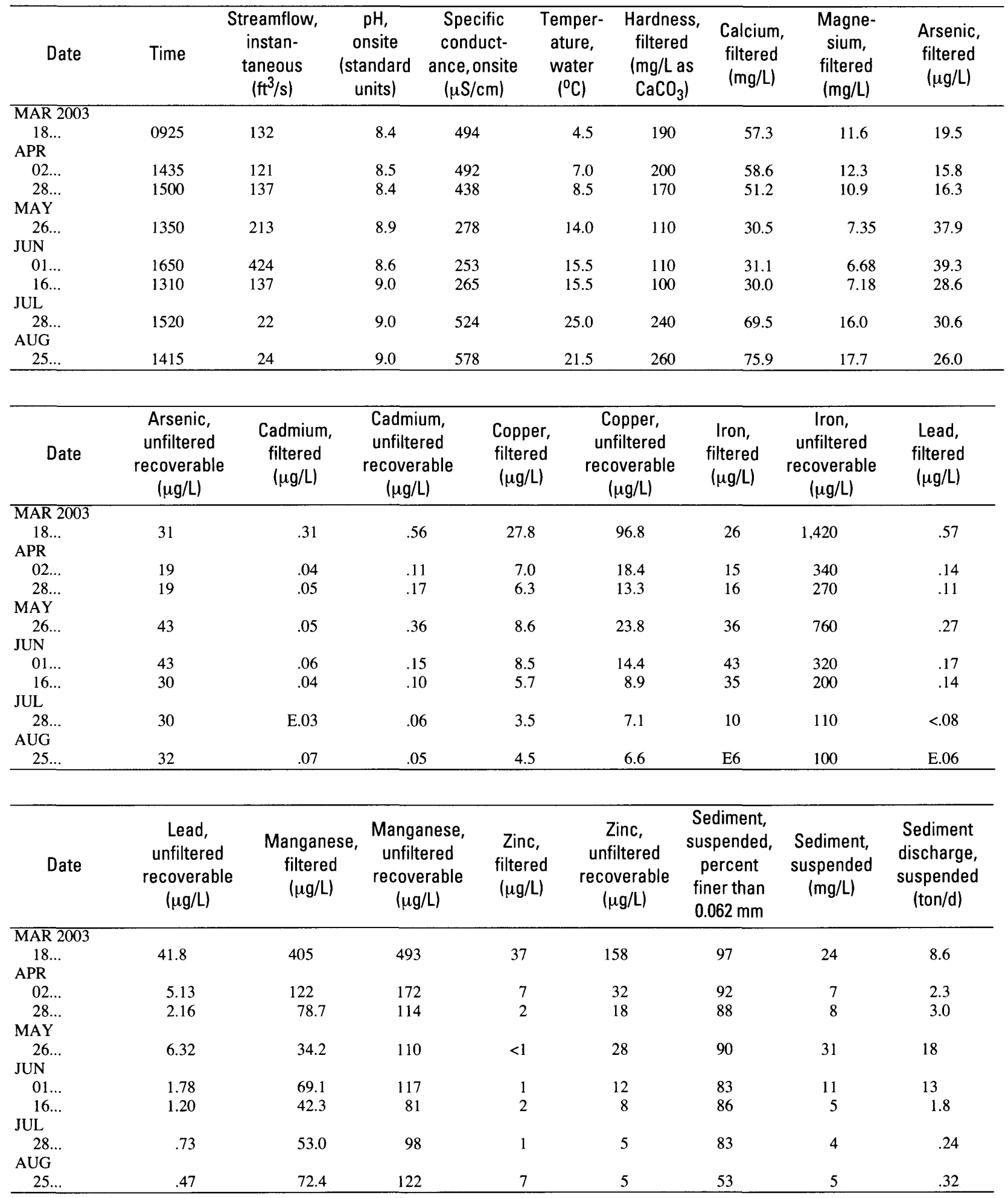


Water-Quality, Bed-Sediment, and Biological Data (October 2002 through September 2003) and Statistical Summaries of Data for Streams in the Upper Clark Fork Basin, Montana

Table 4. Water-quality data for the upper Clark Fork basin, Montana, October 2002 through September 2003-Continued

12323770--WARM SPRINGS CREEK AT WARM SPRINGS, MONT.

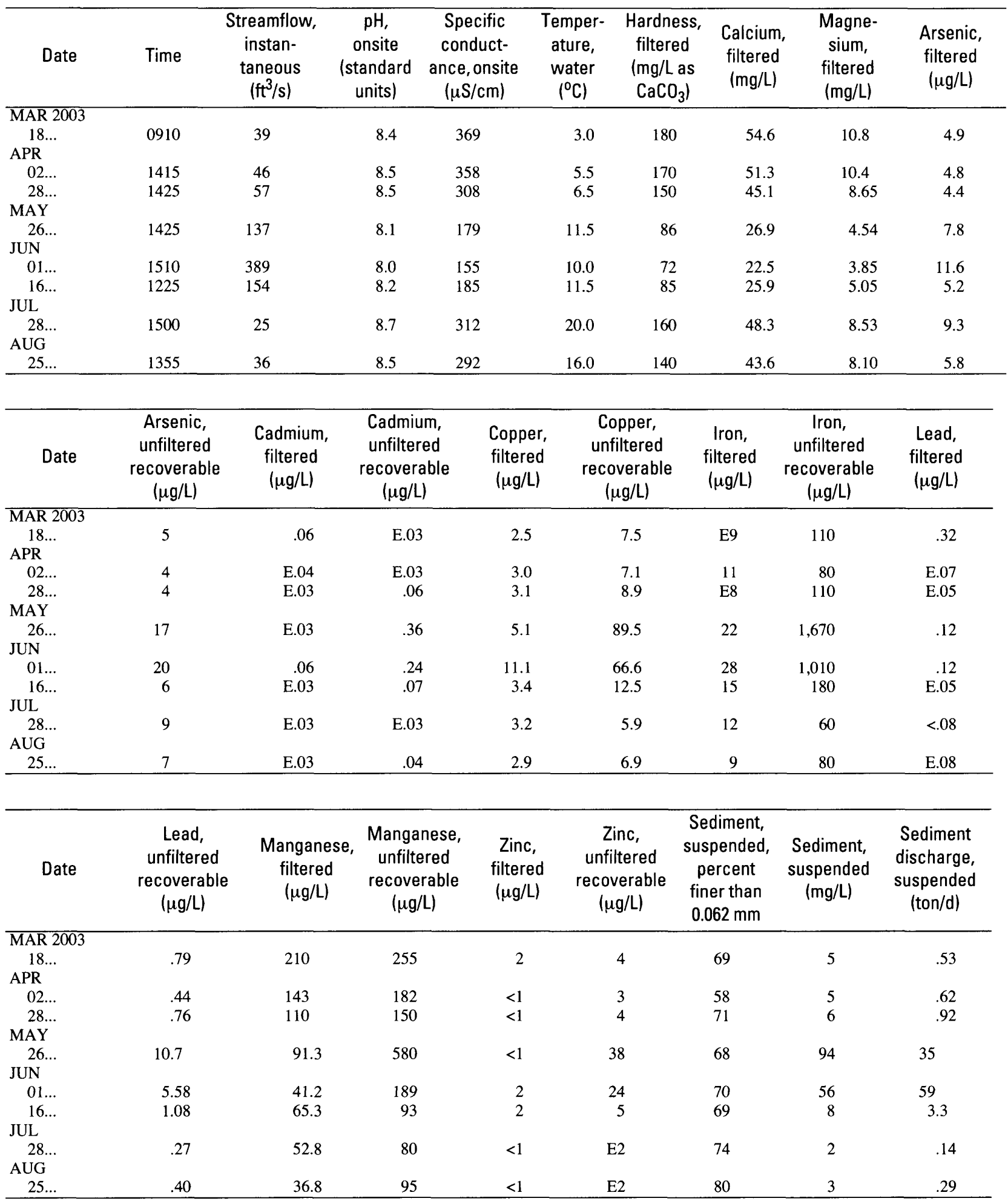


Table 4. Water-quality data for the upper Clark Fork basin, Montana, October 2002 through September 2003-Continued 12323800--CLARK FORK NEAR GALEN, MONT.

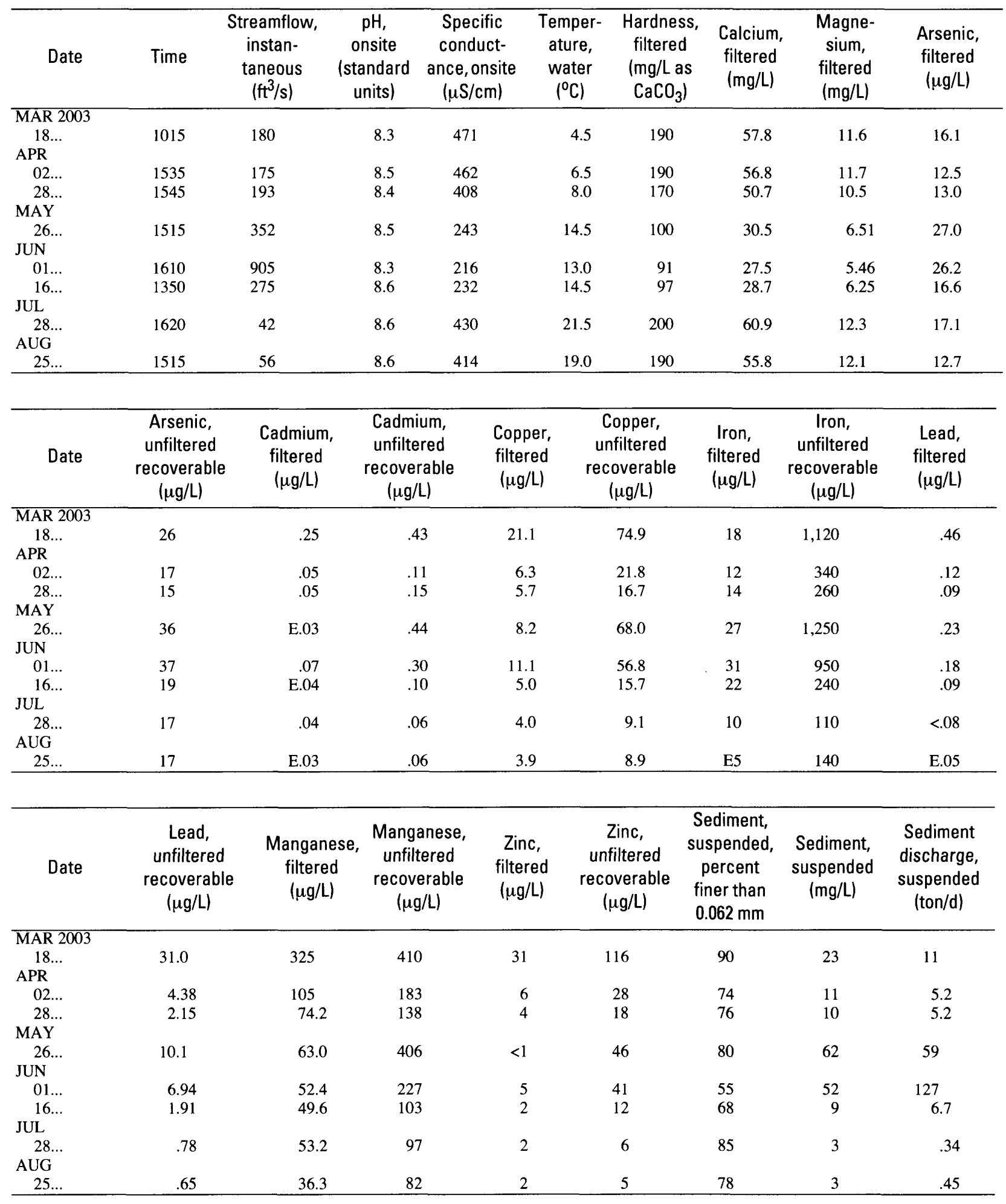


24 Water-Quality, Bed-Sediment, and Biological Data (October 2002 through September 2003) and Statistical Summaries of Data for Streams in the Upper Clark Fork Basin, Montana

Table 4. Water-quality data for the upper Clark Fork basin, Montana, October 2002 through September 2003-Continued 12323850--LOST CREEK NEAR GALEN, MONT.

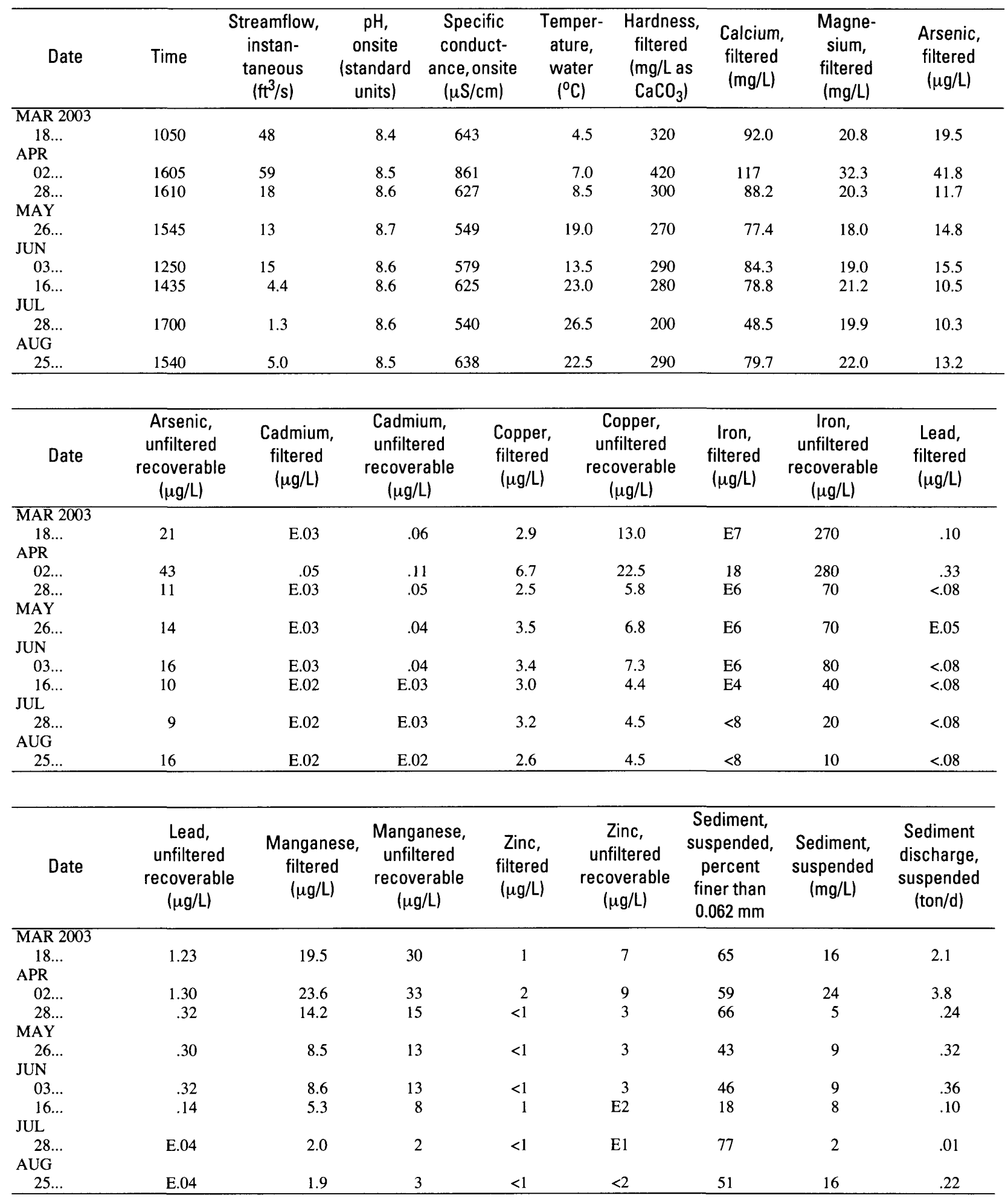


Table 4. Water-quality data for the upper Clark Fork basin, Montana, October 2002 through September 2003-Continued 12324200--CLARK FORK AT DEER LODGE, MONT.

\begin{tabular}{|c|c|c|c|c|c|c|c|c|c|c|}
\hline Date & Time & $\begin{array}{l}\text { Streamflow, } \\
\text { instan- } \\
\text { taneous } \\
\left(\mathrm{ft}^{3} / \mathrm{s}\right)\end{array}$ & $\begin{array}{l}\mathrm{pH}, \\
\text { onsite } \\
\text { standard } \\
\text { units) }\end{array}$ & $\begin{array}{r}\text { Speci } \\
\text { condu } \\
\text { ance, oI } \\
(\mu \mathrm{S} / \mathrm{c}\end{array}$ & & $\begin{array}{l}\text { Temper- } \\
\text { ature, } \\
\text { water } \\
\left({ }^{\circ} \mathrm{C}\right)\end{array}$ & $\begin{array}{c}\text { Hardness, } \\
\text { filtered } \\
\text { (mg/L as } \\
\mathrm{CaCO}_{3} \text { ) }\end{array}$ & $\begin{array}{c}\text { Calcium, } \\
\text { filtered } \\
(\mathrm{mg} / \mathrm{L})\end{array}$ & $\begin{array}{l}\text { Magne- } \\
\text { sium, } \\
\text { filtered } \\
\text { (mg/L) }\end{array}$ & $\begin{array}{c}\text { Arsenic, } \\
\text { filtered } \\
(\mu \mathrm{g} / \mathrm{L})\end{array}$ \\
\hline \multicolumn{11}{|l|}{ MAR 2003} \\
\hline $18 \ldots$ & 1215 & 342 & 8.2 & 515 & & 5.0 & 220 & 64.6 & 13.5 & 16.2 \\
\hline \multicolumn{11}{|l|}{ APR } \\
\hline $03 \ldots$ & 0720 & 347 & 8.2 & 576 & & 3.5 & 250 & 72.4 & 17.2 & 16.7 \\
\hline $28 \ldots$ & 1730 & 282 & 8.6 & 460 & & 9.5 & 190 & 57.2 & 12.3 & 13.7 \\
\hline \multicolumn{11}{|l|}{ MAY } \\
\hline \multirow{2}{*}{\multicolumn{11}{|c|}{ JUN }} \\
\hline & & & & & & & & & & \\
\hline $01 \ldots$ & 1900 & 1,010 & 8.0 & 250 & & 14.0 & 110 & 32.2 & 6.44 & 23.7 \\
\hline $16 \ldots$ & 1515 & 347 & 8.3 & 303 & & 18.5 & 130 & 38.6 & 8.19 & 17.0 \\
\hline \multicolumn{11}{|l|}{ JUL } \\
\hline \multicolumn{11}{|l|}{ AUG } \\
\hline $25 \ldots$ & 1635 & 80 & 8.6 & 495 & & 21.0 & 220 & 64.6 & 13.5 & 15.8 \\
\hline Date & $\begin{array}{c}\text { Arsenic, } \\
\text { unfiltered } \\
\text { recoverable } \\
(\mu \mathrm{g} / \mathrm{L})\end{array}$ & $\begin{array}{l}\text { Cadmium, } \\
\text { filtered } \\
(\mu \mathrm{g} / \mathrm{L})\end{array}$ & $\begin{array}{r}\text { Cadm } \\
\text { unfilte } \\
\text { recove } \\
\text { lug }\end{array}$ & $\begin{array}{l}\text { ium, } \\
\text { ered } \\
\text { rable } \\
\text { /L) }\end{array}$ & $\begin{array}{c}\text { Copper, } \\
\text { filtered } \\
(\mu \mathrm{g} / \mathrm{L})\end{array}$ & & $\begin{array}{l}\text { Copper, } \\
\text { anfiltered } \\
\text { coverable } \\
(\mu \mathrm{g} / \mathrm{L})\end{array}$ & $\begin{array}{l}\text { Iron, } \\
\text { filtered } \\
(\mu \mathrm{g} / \mathrm{L})\end{array}$ & $\begin{array}{c}\text { Iron, } \\
\text { unfiltered } \\
\text { recoverable } \\
(\mu \mathrm{g} / \mathrm{L})\end{array}$ & $\begin{array}{l}\text { Lead, } \\
\text { filtered } \\
(\mu \mathrm{g} / \mathrm{L})\end{array}$ \\
\hline \multicolumn{11}{|l|}{ MAR 2003} \\
\hline $\begin{array}{l}18 \ldots \\
\text { APR }\end{array}$ & \multicolumn{9}{|c|}{ APR } & .25 \\
\hline $03 \ldots$ & 25 & .07 & & 33 & 9.9 & & 66.1 & 18 & 1,040 & .14 \\
\hline $28 \ldots$ & 17 & .06 & & 18 & 7.6 & & 31.1 & E8 & 460 & E. 07 \\
\hline \multicolumn{11}{|l|}{ MAY } \\
\hline $\begin{array}{l}26 \ldots \\
\text { JUN }\end{array}$ & \multicolumn{9}{|c|}{ JUN } & .24 \\
\hline $01 \ldots$ & 53 & .08 & & 88 & 18.7 & & 241 & 44 & 2,910 & .54 \\
\hline $16 \ldots$ & 21 & .06 & & 20 & 9.6 & & 38.3 & 24 & 490 & .14 \\
\hline \multicolumn{11}{|l|}{ JUL } \\
\hline $\begin{array}{l}29 \ldots \\
\text { AUG }\end{array}$ & \multicolumn{9}{|c|}{ AUG } & $<.08$ \\
\hline $25 \ldots$ & 20 & .04 & & 11 & 6.6 & & 17.8 & $<8$ & 150 & E.06 \\
\hline Date & $\begin{array}{c}\text { Lead, } \\
\text { unfiltered } \\
\text { recoverable } \\
(\mu \mathrm{g} / \mathrm{L})\end{array}$ & $\begin{array}{c}\text { Manganese, } \\
\text { filtered } \\
(\mu \mathrm{g} / \mathrm{L})\end{array}$ & $\begin{array}{r}\text { Mang } \\
\text { unfil } \\
\text { recov } \\
(\mu\end{array}$ & $\begin{array}{l}\text { janese, } \\
\text { tered } \\
\text { lerable } \\
\mathrm{g} / \mathrm{L} \text { ) }\end{array}$ & $\begin{array}{c}\text { Zinc, } \\
\text { filtered } \\
(\mu \mathrm{g} / \mathrm{L})\end{array}$ & & $\begin{array}{lr}\text { Zinc, } & \text { S } \\
\text { nfiltered } & \text { st } \\
\text { overable } \\
(\mu \mathrm{g} / \mathrm{L})\end{array}$ & $\begin{array}{l}\text { Sediment, } \\
\text { uspended, } \\
\text { percent } \\
\text { finer than } \\
0.062 \mathrm{~mm}\end{array}$ & $\begin{array}{c}\text { Sediment, } \\
\text { suspended } \\
(\mathrm{mg} / \mathrm{L})\end{array}$ & $\begin{array}{l}\text { Sediment } \\
\text { discharge, } \\
\text { suspended } \\
\text { (ton/d) }\end{array}$ \\
\hline \multicolumn{11}{|l|}{$\overline{\text { MAR } 2003}$} \\
\hline \multicolumn{11}{|l|}{ APR } \\
\hline $03 \ldots$ & 10.2 & 51.2 & 20 & & 9 & & 67 & 57 & 55 & 52 \\
\hline $28 \ldots$ & 4.08 & 27.1 & 11 & & 3 & & 30 & 68 & 22 & 17 \\
\hline \multicolumn{11}{|l|}{ MAY } \\
\hline \multicolumn{11}{|l|}{ JUN } \\
\hline $01 \ldots$ & 33.9 & 40.9 & 40 & & 9 & & 167 & 52 & 187 & 510 \\
\hline $\begin{array}{l}16 \ldots \\
\text { JUL }\end{array}$ & 4.88 & 26.2 & 10 & & 6 & & 32 & 64 & 24 & 22 \\
\hline $\begin{array}{r}29 \ldots \\
\text { AUG }\end{array}$ & .67 & 34.4 & 4 & 8 & 8 & & 13 & 88 & 3 & .32 \\
\hline $25 \ldots$ & 1.46 & 17.1 & 7 & 3 & 3 & & 13 & 69 & 14 & 3.0 \\
\hline
\end{tabular}


26 Water-Quality, Bed-Sediment, and Biological Data (October 2002 through September 2003) and Statistical Summaries of Data for Streams in the Upper Clark Fork Basin, Montana

Table 4. Water-quality data for the upper Clark Fork basin, Montana, October 2002 through September 2003-Continued

12324590--LITTLE BLACKFOOT RIVER NEAR GARRISON, MONT.

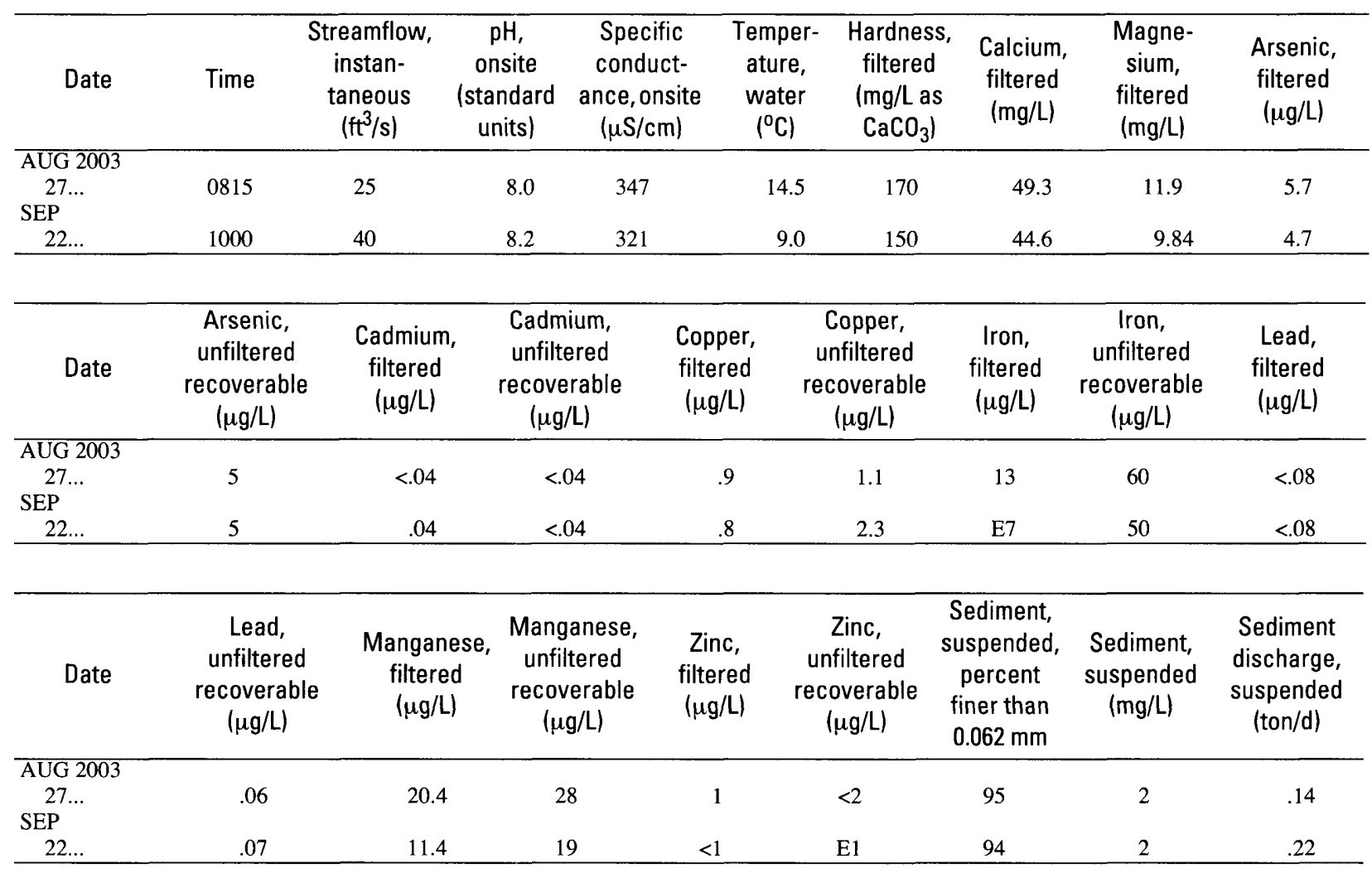


Table 4. Water-quality data for the upper Clark Fork basin, Montana, October 2002 through September 2003-Continued 12324680--CLARK FORK AT GOLDCREEK, MONT.

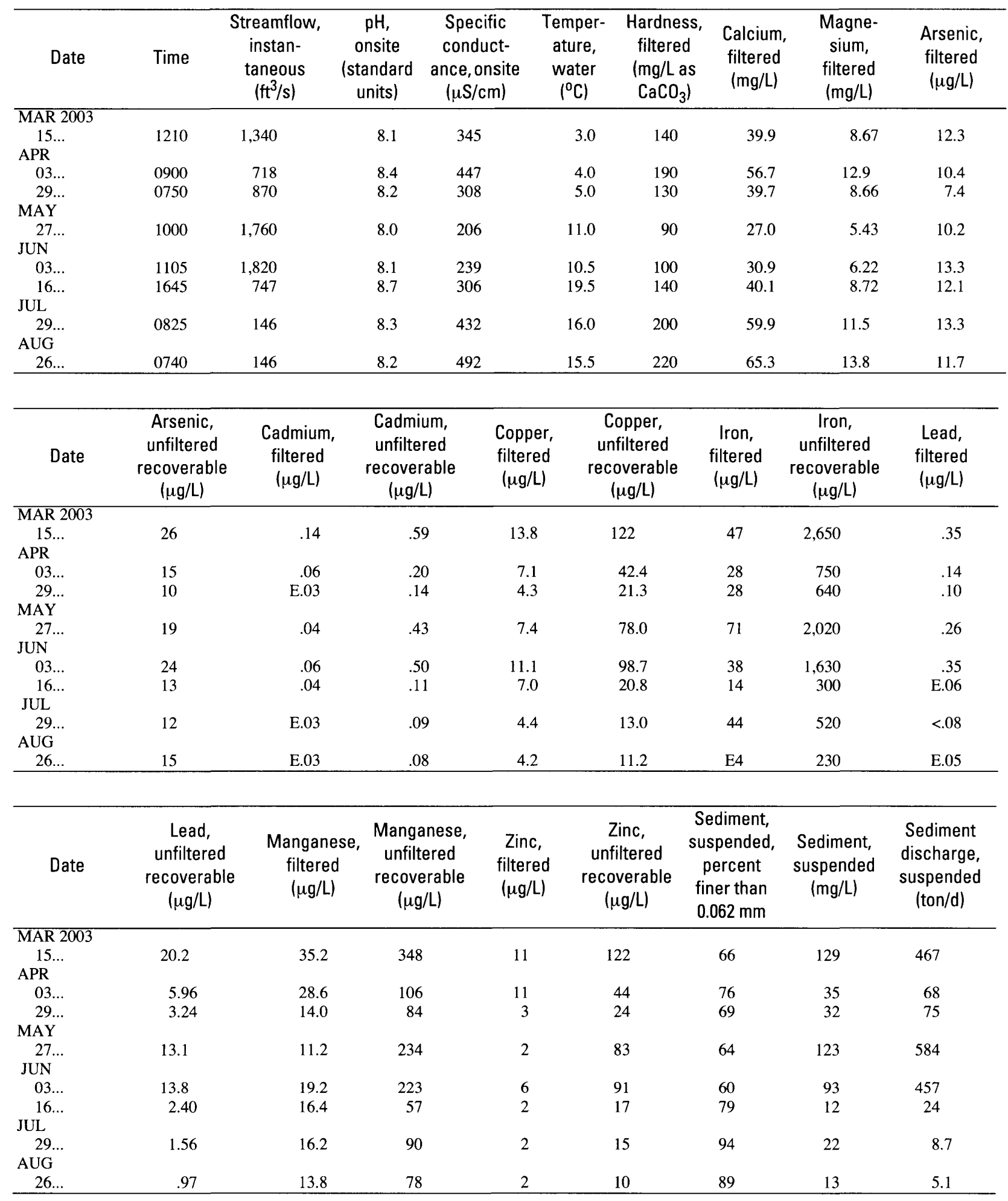


28 Water-Quality, Bed-Sediment, and Biological Data (October 2002 through September 2003) and Statistical Summaries of Data for Streams in the Upper Clark Fork Basin, Montana

Table 4. Water-quality data for the upper Clark Fork basin, Montana, October 2002 through September 2003-Continued 12331500--FLINT CREEK NEAR DRUMMOND, MONT.




Table 4. Water-quality data for the upper Clark Fork basin, Montana, October 2002 through September 2003-Continued 12331800--CLARK FORK NEAR DRUMMOND, MONT.

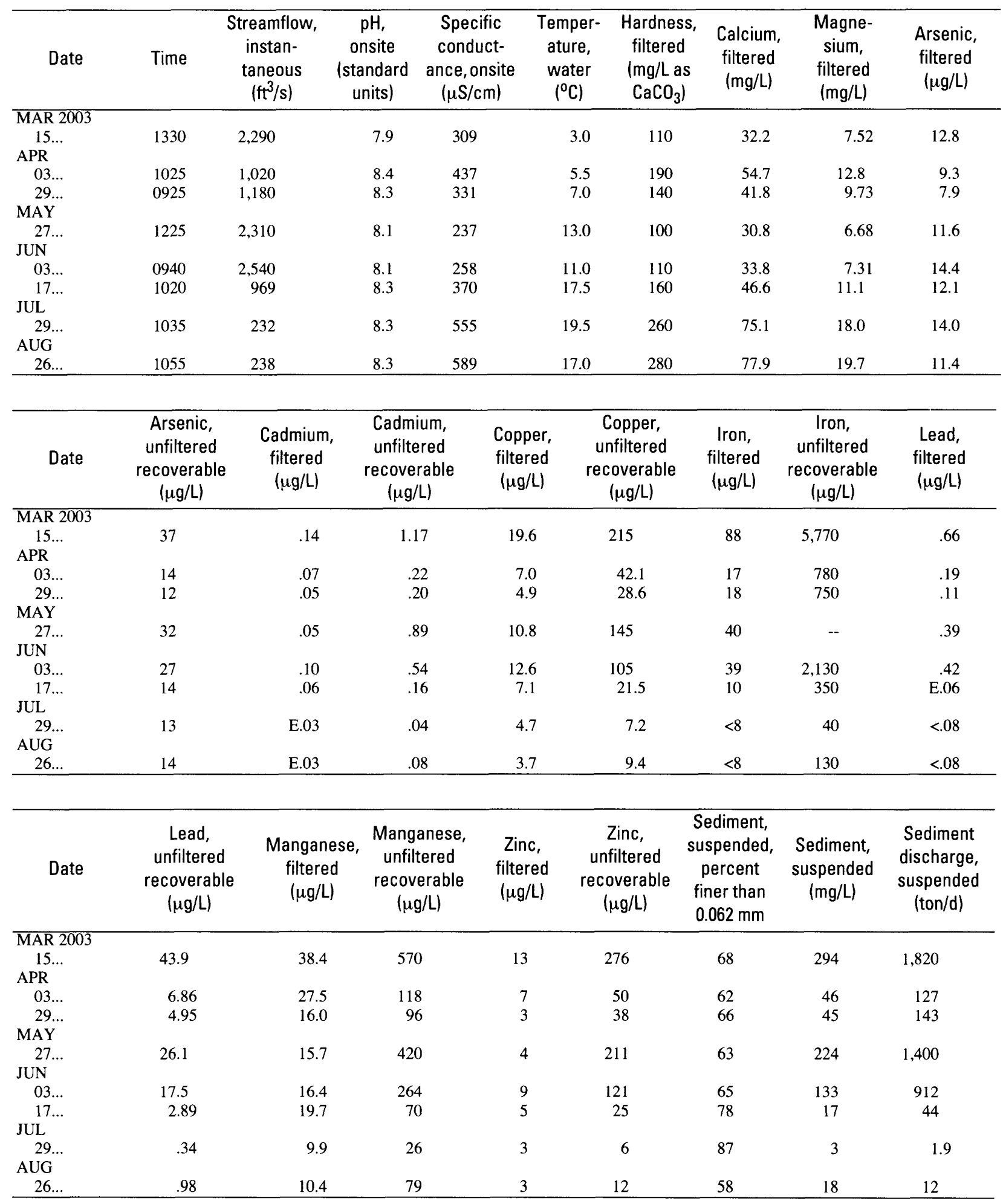


30 Water-Quality, Bed-Sediment, and Biological Data (October 2002 through September 2003) and Statistical Summaries of Data for Streams in the Upper Clark Fork Basin, Montana

Table 4. Water-quality data for the upper Clark Fork basin, Montana, October 2002 through September 2003-Continued 12334510--ROCK CREEK NEAR CLINTON, MONT.

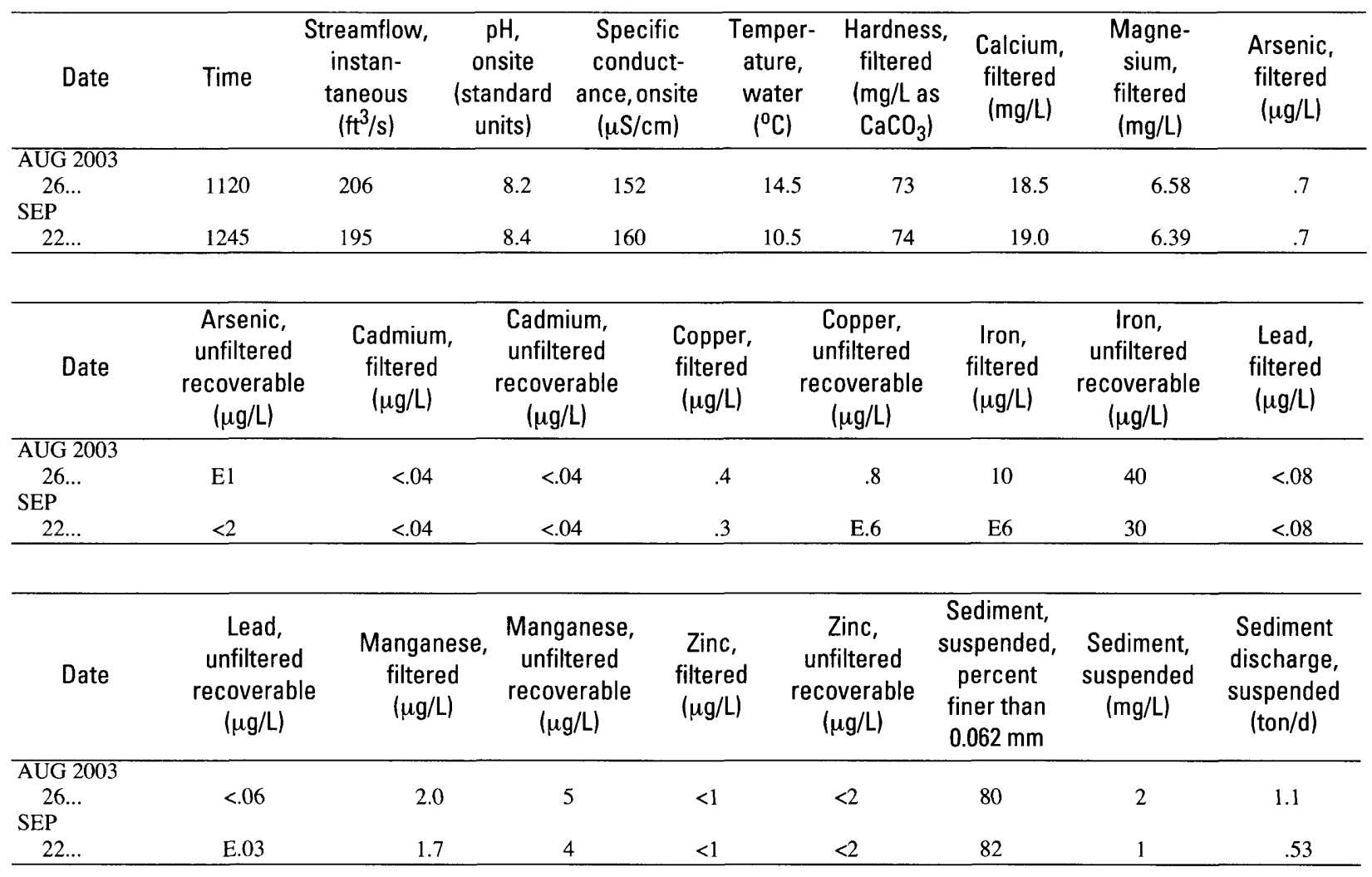


Table 4. Water-quality data for the upper Clark Fork basin, Montana, October 2002 through September 2003-Continued

12334550--CLARK FORK AT TURAH BRIDGE, NEAR BONNER, MONT.

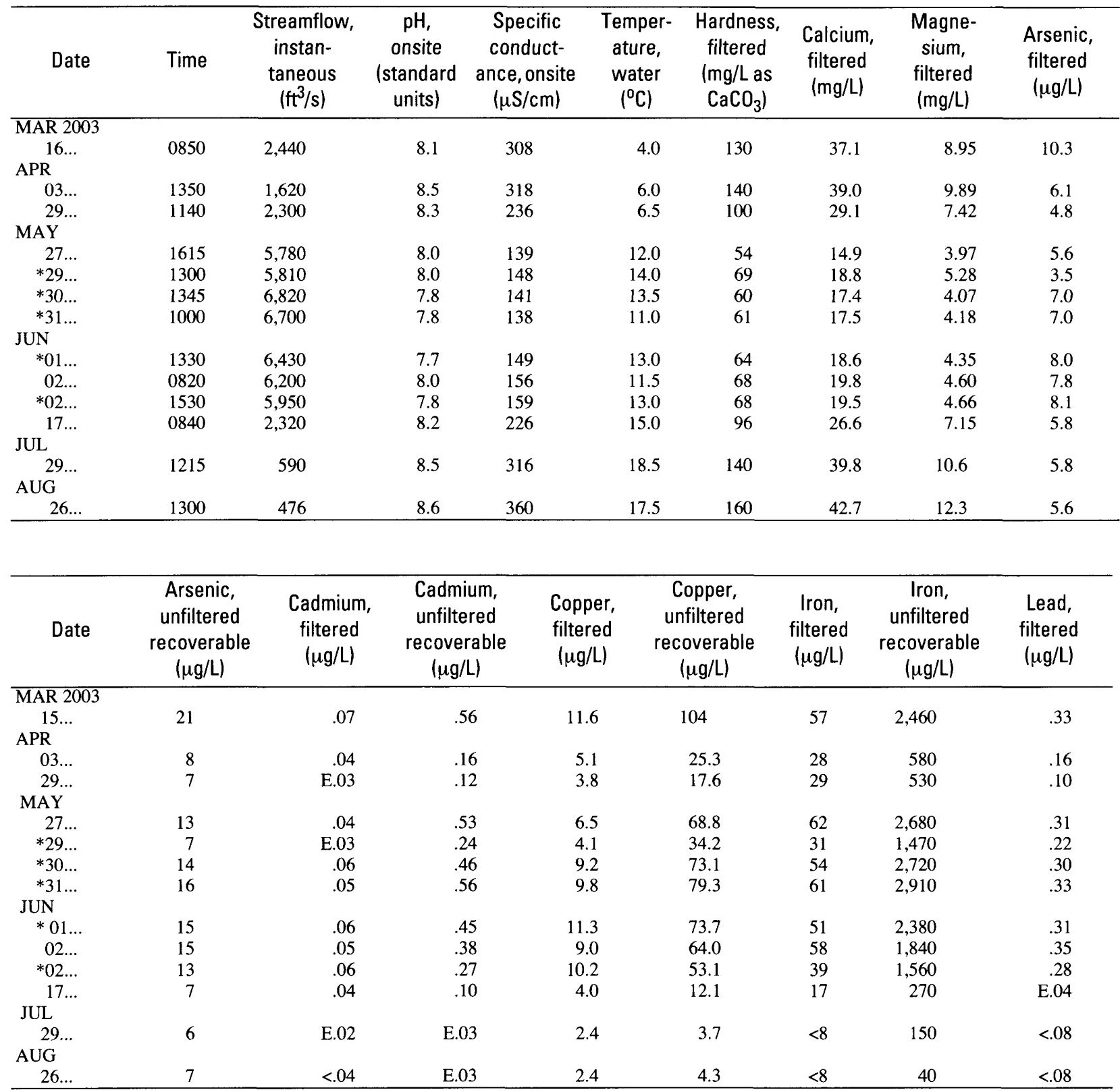


32 Water-Quality, Bed-Sediment, and Biological Data (October 2002 through September 2003) and Statistical Summaries of Data for Streams in the Upper Clark Fork Basin, Montana

Table 4. Water-quality data for the upper Clark Fork basin, Montana, October 2002 through September 2003-Continued

12334550--CLARK FORK AT TURAH BRIDGE, NEAR BONNER, MONT.-Continued

\begin{tabular}{|c|c|c|c|c|c|c|c|c|}
\hline Date & $\begin{array}{c}\text { Lead, } \\
\text { unfiltered } \\
\text { recoverable } \\
(\mu \mathrm{g} / \mathrm{L})\end{array}$ & $\begin{array}{c}\text { Manganese, } \\
\text { filtered } \\
(\mu \mathrm{g} / \mathrm{L})\end{array}$ & $\begin{array}{c}\text { Manganese, } \\
\text { unfiltered } \\
\text { recoverable } \\
(\mu \mathrm{g} / \mathrm{L})\end{array}$ & $\begin{array}{c}\text { Zinc, } \\
\text { filtered } \\
(\mu \mathrm{g} / \mathrm{L})\end{array}$ & $\begin{array}{c}\text { Zinc, } \\
\text { unfiltered } \\
\text { recoverable } \\
(\mu \mathrm{g} / \mathrm{L})\end{array}$ & $\begin{array}{c}\text { Sediment, } \\
\text { suspended, } \\
\text { percent } \\
\text { finer than } \\
0.062 \mathrm{~mm}\end{array}$ & $\begin{array}{l}\text { Sediment, } \\
\text { suspended } \\
\text { (mg/L) }\end{array}$ & $\begin{array}{l}\text { Sediment } \\
\text { discharge, } \\
\text { suspended } \\
\text { (ton/d) }\end{array}$ \\
\hline \multicolumn{9}{|l|}{ MAR 2003} \\
\hline $15 \ldots$ & 17.5 & 14.6 & 278 & 9 & 135 & 78 & 131 & 863 \\
\hline \multicolumn{9}{|l|}{ APR } \\
\hline $03 \ldots$ & 4.02 & 10.1 & 69 & 3 & 35 & 77 & 30 & 131 \\
\hline $29 \ldots$ & 3.19 & 6.4 & 58 & 2 & 27 & 68 & 31 & 193 \\
\hline \multicolumn{9}{|l|}{ MAY } \\
\hline $27 \ldots$ & 14.9 & 15.4 & 248 & 2 & 121 & 55 & 218 & 3,400 \\
\hline$* 29 \ldots$ & 6.52 & 2.1 & 126 & 4 & 62 & -- & 316 & 4,960 \\
\hline$* 30 \ldots$ & 14.2 & 2.0 & 247 & 6 & 118 & -- & 295 & 5,430 \\
\hline$* 31 \ldots$ & 15.8 & 2.4 & 278 & 8 & 143 & -- & 281 & 5,080 \\
\hline \multicolumn{9}{|l|}{ JUN } \\
\hline$* 01 \ldots$ & 14.0 & 2.3 & 231 & 6 & 115 & -- & 222 & 3,850 \\
\hline $02 \ldots$ & 10.8 & 10.2 & 186 & 6 & 88 & 64 & 134 & 2,240 \\
\hline$* 02 \ldots$ & 9.33 & 2.3 & 153 & 6 & 75 & -- & 146 & 2,350 \\
\hline $17 \ldots$ & 1.54 & 12.8 & 39 & 4 & 16 & 66 & 16 & 100 \\
\hline \multicolumn{9}{|l|}{ JUL } \\
\hline $29 \ldots$ & .18 & 5.4 & 13 & 1 & 4 & 84 & 3 & 4.8 \\
\hline \multicolumn{9}{|l|}{ AUG } \\
\hline $26 \ldots$ & .21 & 4.1 & 16 & 2 & 4 & 90 & 3 & 3.9 \\
\hline
\end{tabular}

*Supplemental samples collected by observer during high flow. Samples were collected by depth integration at a single vertical near midstream. 
Table 4. Water-quality data for the upper Clark Fork basin, Montana, October 2002 through September 2003-Continued

12340000--BLACKFOOT RIVER NEAR BONNER, MONT.

\begin{tabular}{lccccccccc}
\hline Date & Time & $\begin{array}{c}\text { Streamflow, } \\
\text { instan- } \\
\text { taneous } \\
\left(\mathrm{ft}^{3} / \mathrm{s}\right)\end{array}$ & $\begin{array}{c}\mathrm{pH}, \\
\text { onsite } \\
(\text { standard } \\
\text { units) }\end{array}$ & $\begin{array}{c}\text { Specific } \\
\text { conduct- } \\
\text { ance, onsite } \\
(\mu \mathrm{S} / \mathrm{cm})\end{array}$ & $\begin{array}{c}\text { Temper- } \\
\text { ature, } \\
\text { water } \\
\left({ }^{\circ} \mathrm{C}\right)\end{array}$ & $\begin{array}{c}\text { Hardness, } \\
\text { filtered } \\
(\mathrm{mg} / \mathrm{L} \text { as } \\
\mathrm{CaCO})\end{array}$ & $\begin{array}{c}\text { Calcium, } \\
\text { filtered } \\
(\mathrm{mg} / \mathrm{L})\end{array}$ & $\begin{array}{c}\text { Magne- } \\
\text { sium, } \\
\text { filtered } \\
(\mathrm{mg} / \mathrm{L})\end{array}$ & $\begin{array}{c}\text { Arsenic, } \\
\text { filtered } \\
(\mu \mathrm{m} / \mathrm{L})\end{array}$ \\
$\begin{array}{c}\text { AUG 2003 } \\
26 \ldots\end{array}$ & 1440 & 512 & 8.6 & 268 & 18.0 & 140 & 33.8 & 13.0 & 1.4 \\
$\begin{array}{c}\mathrm{SEP} \\
22 \ldots\end{array}$ & 1425 & 489 & 8.6 & 271 & 12.5 & 140 & 34.3 & 12.8 & 1.2 \\
\hline
\end{tabular}

\begin{tabular}{|c|c|c|c|c|c|c|c|c|}
\hline Date & $\begin{array}{c}\text { Arsenic, } \\
\text { unfiltered } \\
\text { recoverable } \\
(\mu \mathrm{g} / \mathrm{L})\end{array}$ & $\begin{array}{c}\text { Cadmium, } \\
\text { filtered } \\
(\mu \mathrm{g} / \mathrm{L})\end{array}$ & $\begin{array}{l}\text { Cadmium, } \\
\text { unfiltered } \\
\text { recoverable } \\
(\mu \mathrm{g} / \mathrm{L})\end{array}$ & $\begin{array}{c}\text { Copper, } \\
\text { filtered } \\
(\mu \mathrm{g} / \mathrm{L})\end{array}$ & $\begin{array}{c}\text { Copper, } \\
\text { unfiltered } \\
\text { recoverable } \\
(\mu \mathrm{g} / \mathrm{L})\end{array}$ & $\begin{array}{l}\text { Iron, } \\
\text { filtered } \\
(\mu \mathrm{g} / \mathrm{L})\end{array}$ & $\begin{array}{c}\text { Iron, } \\
\text { unfiltered } \\
\text { recoverable } \\
(\mu \mathrm{g} / \mathrm{L})\end{array}$ & $\begin{array}{c}\text { Lead, } \\
\text { filtered } \\
(\mu \mathrm{g} / \mathrm{L})\end{array}$ \\
\hline \multicolumn{9}{|l|}{$\overline{\text { AUG } 2003}$} \\
\hline $26 \ldots$ & E2 & $<.04$ & $<.04$ & .5 & E.6 & $\mathrm{E} 4$ & 20 & $<.08$ \\
\hline \multicolumn{9}{|l|}{ SEP } \\
\hline $22 \ldots$ & E1 & $<.04$ & $<.04$ & .4 & .6 & $<8$ & 10 & $<.08$ \\
\hline
\end{tabular}

\begin{tabular}{lcccccccc}
\hline Date & $\begin{array}{c}\text { Lead, } \\
\text { unfiltered } \\
\text { recoverable } \\
(\mu \mathrm{g} / \mathrm{L})\end{array}$ & $\begin{array}{c}\text { Manganese, } \\
\text { filtered } \\
(\mu \mathrm{g} / \mathrm{L})\end{array}$ & $\begin{array}{c}\text { Manganese, } \\
\text { unfiltered } \\
\text { recoverable } \\
(\mu \mathrm{g} / \mathrm{L})\end{array}$ & $\begin{array}{c}\text { Zinc, } \\
\text { filtered } \\
(\mu \mathrm{g} / \mathrm{L})\end{array}$ & $\begin{array}{c}\text { Zinc, } \\
\text { unfiltered } \\
\text { recoverable } \\
(\mu \mathrm{g} / \mathrm{L})\end{array}$ & $\begin{array}{c}\text { Sediment, } \\
\text { suspended, } \\
\text { percent } \\
\text { finer than } \\
0.062 \mathrm{~mm}\end{array}$ & $\begin{array}{c}\text { Sediment, } \\
\text { suspended } \\
(\mathrm{mg} / \mathrm{L})\end{array}$ & $\begin{array}{c}\text { Sediment } \\
\text { discharge, } \\
\text { suspended } \\
(\text { ton/d) }\end{array}$ \\
\hline $\begin{array}{c}\text { AUG 2003 } \\
26 \ldots\end{array}$ & $<.06$ & 1.4 & 4 & $<1$ & $<2$ & 79 & 1 & 1.4 \\
$\begin{array}{c}\text { SEP } \\
22 \ldots\end{array}$ & $<.06$ & 1.1 & 3 & 3 & $<2$ & 82 & 1 & 1.3 \\
\hline
\end{tabular}


34 Water-Quality, Bed-Sediment, and Biological Data (October 2002 through September 2003) and Statistical Summaries of Data for Streams in the Upper Clark Fork Basin, Montana

Table 4. Water-quality data for the upper Clark Fork basin, Montana, October 2002 through September 2003_Continued 12340500--CLARK FORK ABOVE MISSOULA, MONT.

\begin{tabular}{|c|c|c|c|c|c|c|c|c|c|}
\hline Date & Time & $\begin{array}{l}\text { Streamflow, } \\
\text { instan- } \\
\text { taneous } \\
\left(\mathrm{ft}^{3} / \mathrm{s}\right)\end{array}$ & $\begin{array}{c}\mathrm{pH}, \\
\text { Onsite } \\
\text { (standard } \\
\text { units) }\end{array}$ & $\begin{array}{c}\text { Specific } \\
\text { conduct- } \\
\text { ance, onsite } \\
(\mu \mathrm{S} / \mathrm{cm})\end{array}$ & $\begin{array}{c}\text { Temper- } \\
\text { ature, } \\
\text { water } \\
\left({ }^{\circ} \mathrm{C}\right)\end{array}$ & $\begin{array}{l}\text { Hardness, } \\
\text { filtered } \\
(\mathrm{mg} / \mathrm{L} \text { as } \\
\left.\mathrm{CaCO}_{3}\right)\end{array}$ & $\begin{array}{c}\text { Calcium, } \\
\text { filtered } \\
(\mathrm{mg} / \mathrm{L})\end{array}$ & $\begin{array}{l}\text { Magne- } \\
\text { sium, } \\
\text { filtered } \\
\text { (mg/L) }\end{array}$ & $\begin{array}{c}\text { Arsenic, } \\
\text { filtered } \\
(\mu \mathrm{g} / \mathrm{L})\end{array}$ \\
\hline MAR 2003 & & & & & & & & & \\
\hline $15 \ldots$ & 1540 & 5,140 & 7.9 & 233 & 4.0 & 93 & 25.9 & 6.78 & 9.0 \\
\hline \multicolumn{10}{|l|}{ APR } \\
\hline $04 \ldots$ & 0800 & 3,290 & 8.3 & 271 & 5.0 & 120 & 32.2 & 9.15 & 3.6 \\
\hline $29 \ldots$ & 1350 & 6,150 & 8.3 & 193 & 7.0 & 89 & 23.9 & 7.07 & 2.3 \\
\hline \multicolumn{10}{|l|}{ MAY } \\
\hline $28 \ldots$ & 0910 & 12,800 & 8.0 & 148 & 12.0 & 71 & 19.4 & 5.34 & 3.4 \\
\hline$* 29 \ldots$ & 1215 & 13,500 & 7.9 & 140 & 14.0 & 61 & 17.6 & 4.25 & 6.2 \\
\hline *30... & 1300 & 14,700 & 7.9 & 147 & 13.0 & 67 & 18.6 & 5.01 & 4.0 \\
\hline$* 31 \ldots$ & 1100 & 15,100 & 7.9 & 145 & 11.0 & 67 & 18.6 & 5.06 & 4.2 \\
\hline \multicolumn{10}{|l|}{ JUN } \\
\hline$* 01 \ldots$ & 1230 & 14,700 & 7.8 & 151 & 11.5 & 69 & 19.1 & 5.11 & 4.8 \\
\hline $02 \ldots$ & 0945 & 13,500 & 8.1 & 156 & 11.5 & 74 & 20.7 & 5.54 & 4.6 \\
\hline$* 02 \ldots$ & 1400 & 13,400 & 7.8 & 159 & 12.0 & 73 & 20.3 & 5.54 & 4.8 \\
\hline $17 \ldots$ & 0720 & 5,470 & 8.3 & 200 & 15.5 & 91 & 23.8 & 7.57 & 3.3 \\
\hline \multicolumn{10}{|l|}{ JUL } \\
\hline $\begin{array}{l}29 \ldots \\
\text { AUG }\end{array}$ & 1335 & 1,280 & 8.6 & 278 & 21.0 & 130 & 34.6 & 10.9 & 4.0 \\
\hline $26 \ldots$ & 1600 & 999 & 8.5 & 311 & 19.5 & 150 & 38.0 & 12.7 & 3.6 \\
\hline
\end{tabular}

\begin{tabular}{|c|c|c|c|c|c|c|c|c|}
\hline Date & $\begin{array}{c}\text { Arsenic, } \\
\text { unfiltered } \\
\text { recoverable } \\
(\mu \mathrm{g} / \mathrm{L})\end{array}$ & $\begin{array}{c}\text { Cadmium, } \\
\text { filtered } \\
(\mu \mathrm{g} / \mathrm{L})\end{array}$ & $\begin{array}{c}\text { Cadmium, } \\
\text { unfiltered } \\
\text { recoverable } \\
(\mu \mathrm{g} / \mathrm{L})\end{array}$ & $\begin{array}{l}\text { Copper, } \\
\text { filtered } \\
(\mu \mathrm{g} / \mathrm{L})\end{array}$ & $\begin{array}{c}\text { Copper, } \\
\text { unfiltered } \\
\text { recoverable } \\
(\mu \mathrm{g} / \mathrm{L})\end{array}$ & $\begin{array}{c}\text { Iron, } \\
\text { filtered } \\
\text { ( } \mu \mathrm{g} / \mathrm{L})\end{array}$ & $\begin{array}{c}\text { Iron, } \\
\text { unfiltered } \\
\text { recoverable } \\
\{\mu \mathrm{g} / \mathrm{L})\end{array}$ & $\begin{array}{c}\text { Lead, } \\
\text { filtered } \\
(\mu \mathrm{g} / \mathrm{L})\end{array}$ \\
\hline \multicolumn{9}{|l|}{ MAR 2003} \\
\hline $15 \ldots$ & 19 & .08 & .64 & 12.6 & 107 & 77 & 3,040 & .34 \\
\hline \multicolumn{9}{|l|}{ APR } \\
\hline $04 \ldots$ & 5 & E.02 & .11 & 3.4 & 18.6 & 47 & 490 & .15 \\
\hline $29 \ldots$ & 3 & E.02 & .09 & 2.3 & 10.5 & 26 & 420 & .08 \\
\hline \multicolumn{9}{|l|}{ MAY } \\
\hline $28 \ldots$ & 8 & E. 02 & .27 & 3.8 & 39.8 & 40 & 1,680 & .17 \\
\hline$* 29 \ldots$ & 13 & .04 & .40 & 7.6 & 68.0 & 49 & 2,430 & .29 \\
\hline$* 30 \ldots$ & 7 & E.03 & .20 & 4.9 & 35.4 & 32 & 1,610 & .17 \\
\hline$* 31 \ldots$ & 9 & .04 & .29 & 5.0 & 45.7 & 38 & 1,910 & .23 \\
\hline \multicolumn{9}{|l|}{ JUN } \\
\hline$* 01 \ldots$ & 9 & E. 03 & .29 & 6.2 & 46.1 & 34 & 1,740 & .20 \\
\hline $02 \ldots$ & 9 & E.03 & .32 & 5.1 & 46.0 & 41 & 1,480 & .22 \\
\hline$* 02 \ldots$ & 9 & .05 & .22 & 5.7 & 36.9 & 26 & 1,320 & .17 \\
\hline $17 \ldots$ & 4 & E.02 & .07 & 2.8 & 8.5 & 13 & 230 & $<.08$ \\
\hline \multicolumn{9}{|l|}{ JUL } \\
\hline $\begin{array}{l}29 \ldots \\
\text { AUG }\end{array}$ & 4 & $<.04$ & E.02 & 1.9 & 3.5 & $<8$ & 70 & $<.08$ \\
\hline $26 \ldots$ & 4 & $<.04$ & E.03 & 2.0 & 4.3 & E5 & 70 & $<.08$ \\
\hline
\end{tabular}


Table 4. Water-quality data for the upper Clark Fork basin, Montana, October 2002 through September 2003-Continued 12340500--CLARK FORK ABOVE MISSOULA, MONT. —Continued

\begin{tabular}{|c|c|c|c|c|c|c|c|c|}
\hline Date & $\begin{array}{c}\text { Lead, } \\
\text { unfiltered } \\
\text { recoverable } \\
(\mu \mathrm{g} / \mathrm{L})\end{array}$ & $\begin{array}{c}\text { Manganese, } \\
\text { filtered } \\
(\mu \mathrm{g} / \mathrm{L})\end{array}$ & $\begin{array}{c}\text { Manganese, } \\
\text { unfiltered } \\
\text { recoverable } \\
(\mu \mathrm{g} / \mathrm{L})\end{array}$ & $\begin{array}{c}\text { Zinc, } \\
\text { filtered } \\
(\mu \mathrm{g} / \mathrm{L})\end{array}$ & $\begin{array}{c}\text { Zinc, } \\
\text { unfiltered } \\
\text { recoverable } \\
(\mu \mathrm{g} / \mathrm{L})\end{array}$ & $\begin{array}{c}\text { Sediment, } \\
\text { suspended, } \\
\text { percent } \\
\text { finer than } \\
0.062 \mathrm{~mm}\end{array}$ & $\begin{array}{l}\text { Sediment, } \\
\text { suspended } \\
\text { (mg/L) }\end{array}$ & $\begin{array}{c}\text { Sediment } \\
\text { discharge, } \\
\text { suspended } \\
\text { (ton/d) }\end{array}$ \\
\hline \multicolumn{9}{|l|}{ MAR 2003} \\
\hline $15 \ldots$ & 17.8 & 21.2 & 314 & 8 & 155 & 85 & 169 & 2,350 \\
\hline \multicolumn{9}{|l|}{ APR } \\
\hline $04 \ldots$ & 2.97 & 22.6 & 53 & 3 & 28 & 82 & 26 & 231 \\
\hline $\begin{array}{l}29 \ldots \\
\text { MAY }\end{array}$ & 1.78 & 13.2 & 42 & 1 & 16 & 85 & 29 & 482 \\
\hline $28 \ldots$ & 7.50 & 13.2 & 143 & 1 & 77 & 75 & 137 & 4,730 \\
\hline$* 29 \ldots$ & 12.8 & 3.1 & 218 & 4 & 105 & -- & 137 & 4,990 \\
\hline$* 30 \ldots$ & 6.87 & 1.6 & 136 & 3 & 68 & -. & 169 & 6,710 \\
\hline$* 31 \ldots$ & 9.14 & 2.0 & 157 & 8 & 85 & -- & 174 & 7,090 \\
\hline \multicolumn{9}{|l|}{ JUN } \\
\hline$* 01 \ldots$ & 8.65 & 1.9 & 154 & 6 & 81 & -- & 168 & 6,670 \\
\hline $02 \ldots$ & 7.93 & 14.2 & 137 & 4 & 79 & 68 & 123 & 4,480 \\
\hline$* 02 \ldots$ & 6.72 & 1.7 & 119 & 4 & 64 & -- & 122 & 4,410 \\
\hline \multirow{2}{*}{\multicolumn{9}{|c|}{ JUL }} \\
\hline & & & & & & & & \\
\hline $\begin{array}{l}29 \ldots \\
\text { AUG }\end{array}$ & .32 & 15.9 & 28 & 1 & 4 & 82 & 5 & 17 \\
\hline $26 \ldots$ & .39 & 14.5 & 28 & 1 & 5 & 89 & 3 & 8.1 \\
\hline
\end{tabular}


36 Water-Quality, Bed-Sediment, and Biological Data (October 2002 through September 2003) and Statistical Summaries of Data for Streams in the Upper Clark Fork Basin, Montana

Table 5. Daily streamflow and suspended-sediment data for Clark Fork at Deer Lodge, Montana, October 2002 through September 2003 [Abbreviations: $\mathrm{ft}^{3} / \mathrm{s}$, cubic feet per second; e, estimated; $\mathrm{mg} / \mathrm{L}$, milligrams per liter; ton/d, tons per day. Symbol: --, no data]

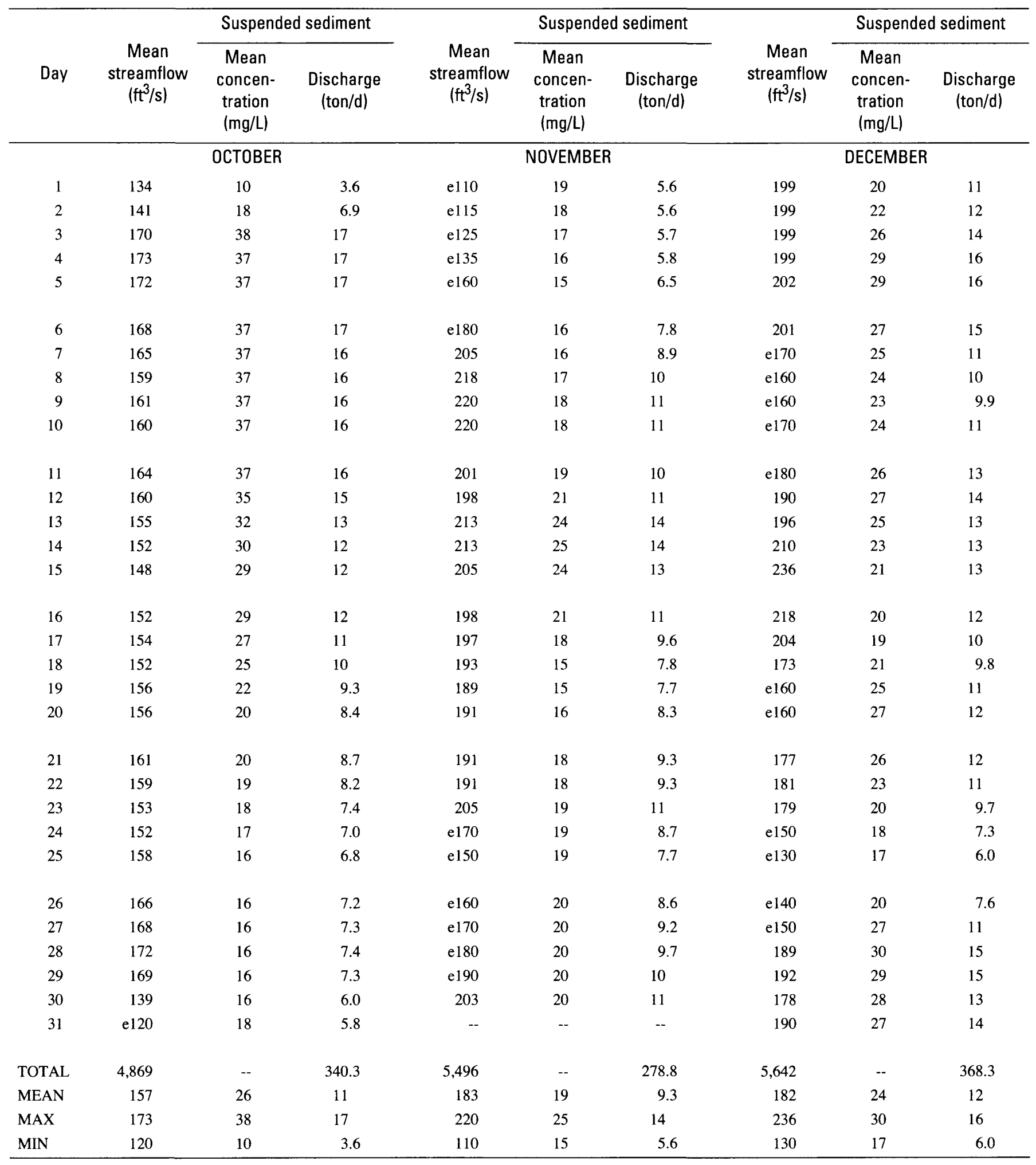


TABLE 537

Table 5. Daily streamflow and suspended-sediment data for Clark Fork at Deer Lodge, Montana, October 2002 through September 2003-Continued

\begin{tabular}{|c|c|c|c|c|c|c|c|c|c|}
\hline \multirow[b]{2}{*}{ Day } & \multirow[b]{2}{*}{$\begin{array}{c}\text { Mean } \\
\text { streamflow } \\
\left(\mathrm{ft}^{3} / \mathrm{s}\right)\end{array}$} & \multicolumn{2}{|c|}{ Suspended sediment } & \multirow[b]{2}{*}{$\begin{array}{c}\text { Mean } \\
\text { streamflow } \\
\left(\mathrm{ft}^{3} / \mathrm{s} \mid\right.\end{array}$} & \multicolumn{2}{|c|}{ Suspended sediment } & \multirow[b]{2}{*}{$\begin{array}{c}\text { Mean } \\
\text { streamflow } \\
\left(\mathrm{ft}^{3} / \mathrm{s}\right)\end{array}$} & \multicolumn{2}{|c|}{ Suspended sediment } \\
\hline & & $\begin{array}{l}\text { Mean } \\
\text { concen- } \\
\text { tration } \\
\text { (mg/L) }\end{array}$ & $\begin{array}{c}\text { Discharge } \\
\text { (ton/d }\}\end{array}$ & & $\begin{array}{c}\text { Mean } \\
\text { concen- } \\
\text { tration } \\
(\mathrm{mg} / \mathrm{L})\end{array}$ & $\begin{array}{l}\text { Discharge } \\
\text { (ton/d) }\end{array}$ & & $\begin{array}{c}\text { Mean } \\
\text { concen- } \\
\text { tration } \\
\text { (mg/L) }\end{array}$ & $\begin{array}{l}\text { Discharge } \\
\text { (ton/d) }\end{array}$ \\
\hline & & JANUARY & & & FEBRUAR & & & MARCH & \\
\hline 1 & 186 & 26 & 13 & 416 & 50 & 56 & 165 & 55 & 25 \\
\hline 2 & 187 & 24 & 12 & 353 & 45 & 43 & 176 & 55 & 26 \\
\hline 3 & 199 & 23 & 12 & 287 & 40 & 31 & 177 & 31 & 15 \\
\hline 4 & 207 & 21 & 12 & 269 & 37 & 27 & 176 & 34 & 16 \\
\hline 5 & 219 & 20 & 12 & 250 & 32 & 22 & 189 & 39 & 20 \\
\hline 6 & 199 & 18 & 9.7 & 229 & 29 & 18 & 186 & 38 & 19 \\
\hline 7 & 180 & 16 & 7.8 & 201 & 27 & 15 & 186 & 33 & 17 \\
\hline 8 & e170 & 15 & 6.9 & 223 & 28 & 17 & 188 & 34 & 17 \\
\hline 9 & e150 & 13 & 5.3 & 220 & 28 & 17 & 189 & 37 & 19 \\
\hline 10 & e140 & 12 & 4.5 & 214 & 28 & 16 & 242 & 59 & 39 \\
\hline 11 & e130 & 13 & 4.6 & 200 & 32 & 17 & 417 & 170 & 191 \\
\hline 12 & e140 & 16 & 6.0 & 197 & 35 & 19 & 601 & 252 & 409 \\
\hline 13 & 185 & 18 & 9.0 & 191 & 29 & 15 & 661 & 197 & 352 \\
\hline 14 & 186 & 19 & 9.5 & 196 & 23 & 12 & 634 & 127 & 217 \\
\hline 15 & 188 & 18 & 9.1 & 191 & 23 & 12 & 589 & 163 & 259 \\
\hline 16 & 172 & 16 & 7.4 & 191 & 26 & 13 & 560 & 104 & 157 \\
\hline 17 & el60 & 15 & 6.5 & 192 & 29 & 15 & 481 & 45 & 58 \\
\hline 18 & e170 & 15 & 6.9 & 189 & 31 & 16 & 344 & 51 & 47 \\
\hline 19 & e180 & 16 & 7.8 & 178 & 32 & 15 & 283 & 43 & 33 \\
\hline 20 & 193 & 16 & 8.3 & 179 & 32 & 15 & 245 & 47 & 31 \\
\hline 21 & e150 & 17 & 6.9 & 179 & 32 & 15 & 237 & 33 & 21 \\
\hline 22 & el30 & 18 & 6.3 & 196 & 31 & 16 & 233 & 32 & 20 \\
\hline 23 & e160 & 28 & 12 & el40 & 31 & 12 & 237 & 28 & 18 \\
\hline 24 & 227 & 38 & 23 & e100 & 38 & 10 & 228 & 27 & 17 \\
\hline 25 & 202 & 41 & 22 & el20 & 48 & 16 & 214 & 26 & 15 \\
\hline 26 & 249 & 66 & 44 & e140 & 57 & 22 & 218 & 24 & 14 \\
\hline 27 & 520 & 300 & 421 & e160 & 52 & 22 & 217 & 19 & 11 \\
\hline 28 & 315 & 87 & 74 & e170 & 52 & 24 & 217 & 17 & 10 \\
\hline 29 & 266 & 51 & 37 & -- & -- & -- & 213 & 22 & 13 \\
\hline 30 & 262 & 44 & 31 & -- & -- & -- & 208 & 24 & 13 \\
\hline 31 & 311 & 42 & 35 & -- & -- & -- & 210 & 22 & 12 \\
\hline TOTAL & 6,333 & -- & 882.5 & 5,771 & -- & 548 & 9,121 & -- & 2,131 \\
\hline MEAN & 204 & 35 & 28 & 206 & 35 & 20 & 294 & 61 & 69 \\
\hline MAX & 520 & 300 & 421 & 416 & 57 & 56 & 661 & 252 & 409 \\
\hline MIN & 130 & 12 & 4.5 & 100 & 23 & 10 & 165 & 17 & 10 \\
\hline
\end{tabular}


38 Water-Quality, Bed-Sediment, and Biological Data (October 2002 through September 2003) and Statistical Summaries of Data for Streams in the Upper Clark Fork Basin, Montana

Table 5. Daily streamflow and suspended-sediment data for Clark Fork at Deer Lodge, Montana, October 2002 through September 2003-Continued

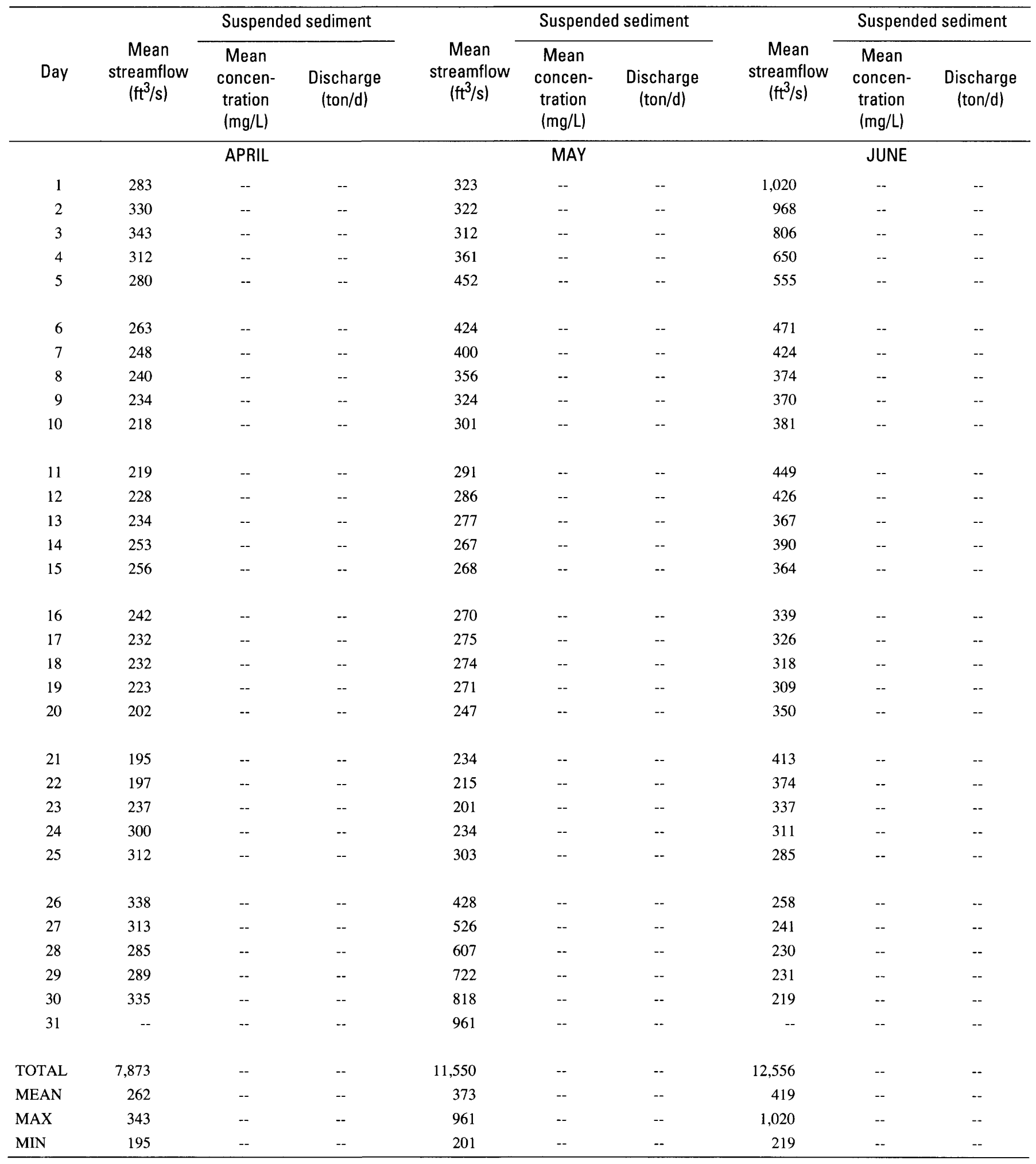


TABLE 539

Table 5. Daily streamflow and suspended-sediment data for Clark Fork at Deer Lodge, Montana, October 2002 through September 2003-Continued

\begin{tabular}{|c|c|c|c|c|c|c|c|c|c|}
\hline \multirow[b]{2}{*}{ Day } & \multirow[b]{2}{*}{$\begin{array}{c}\text { Mean } \\
\text { streamflow } \\
\left(\mathrm{ft}^{3} / \mathrm{s}\right)\end{array}$} & \multicolumn{2}{|c|}{ Suspended sediment } & \multirow[b]{2}{*}{$\begin{array}{c}\text { Mean } \\
\text { streamflow } \\
\left(\mathrm{ft}^{3} / \mathrm{s}\right)\end{array}$} & \multicolumn{2}{|c|}{ Suspended sediment } & \multirow[b]{2}{*}{$\begin{array}{c}\text { Mean } \\
\text { streamflow } \\
\left(\mathrm{ft}^{3} / \mathrm{s}\right)\end{array}$} & \multicolumn{2}{|c|}{ Suspended sediment } \\
\hline & & $\begin{array}{l}\text { Mean } \\
\text { concen- } \\
\text { tration } \\
(\mathrm{mg} / \mathrm{L})\end{array}$ & $\begin{array}{c}\text { Discharge } \\
\text { (ton/d) }\end{array}$ & & $\begin{array}{l}\text { Mean } \\
\text { concen- } \\
\text { tration } \\
(\mathrm{mg} / \mathrm{L})\end{array}$ & $\begin{array}{l}\text { Discharge } \\
\text { (ton/d) }\end{array}$ & & $\begin{array}{l}\text { Mean } \\
\text { concen- } \\
\text { tration } \\
(\mathrm{mg} / \mathrm{L})\end{array}$ & $\begin{array}{c}\text { Discharge } \\
\text { (ton/d) }\end{array}$ \\
\hline & \multicolumn{3}{|c|}{ JULY } & \multicolumn{3}{|c|}{ AUGUST } & \multicolumn{3}{|c|}{ SEPTEMBER } \\
\hline 3 & 182 & -- & -- & 37 & 4 & .40 & 86 & 10 & 2.3 \\
\hline 4 & 169 & -- & -- & 40 & 7 & .76 & 88 & 10 & 2.4 \\
\hline 5 & 161 & -- & -- & 45 & 9 & 1.1 & 87 & 10 & 2.3 \\
\hline 6 & 154 & -- & -- & 45 & 10 & 1.2 & 88 & 10 & 2.4 \\
\hline 10 & 120 & -- & -- & 46 & 10 & 1.2 & 88 & 8 & 1.9 \\
\hline 11 & 104 & -- & -- & 43 & 9 & 1.0 & 88 & 8 & 1.9 \\
\hline 12 & 92 & -- & -- & 45 & 9 & 1.1 & 87 & 9 & 2.1 \\
\hline 13 & 86 & -- & -- & 49 & 10 & 1.3 & 91 & 9 & 2.2 \\
\hline 14 & 84 & -- & -- & 49 & 12 & 1.6 & 94 & 9 & 2.3 \\
\hline 15 & 75 & -- & -- & 49 & 13 & 1.7 & 94 & 9 & 2.3 \\
\hline 16 & 65 & -- & -- & 49 & 14 & 1.9 & 93 & 8 & 2.0 \\
\hline 17 & 59 & -- & -- & 45 & 14 & 1.7 & 106 & 10 & 2.9 \\
\hline 18 & 55 & -- & -- & 57 & 16 & 2.5 & 114 & 16 & 4.9 \\
\hline 25 & 40 & -- & -- & 81 & 14 & 3.1 & 137 & 20 & 7.4 \\
\hline 26 & 42 & -- & -- & 74 & 13 & 2.6 & 130 & 21 & 7.4 \\
\hline 27 & 45 & -- & -- & 76 & 10 & 2.1 & 117 & 21 & 6.6 \\
\hline 28 & 46 & -- & -- & 88 & 10 & 2.4 & 114 & 22 & 6.8 \\
\hline 29 & 40 & -- & -- & 85 & 10 & 2.3 & 112 & 22 & 6.7 \\
\hline 30 & 38 & -- & -- & 94 & 10 & 2.5 & 111 & 23 & 6.9 \\
\hline 31 & 36 & -- & -- & 95 & 10 & 2.6 & -- & -- & -- \\
\hline TOTAL & 2,801 & -- & -- & 1,841 & -- & 53.57 & 3,031 & -- & 119.7 \\
\hline MEAN & 90.4 & -- & -- & 59.4 & 10 & 1.7 & 101 & 14 & 4.0 \\
\hline MAX & 198 & -- & -- & 95 & 16 & 3.1 & 137 & 23 & 7.4 \\
\hline MIN & 36 & -- & - & 37 & 3 & .31 & 86 & 8 & 1.9 \\
\hline
\end{tabular}

TOTAL FOR WATER YEAR 2003: 
40 Water-Quality, Bed-Sediment, and Biological Data (October 2002 through September 2003) and Statistical Summaries of Data for Streams in the Upper Clark Fork Basin, Montana

Table 6. Daily streamflow and suspended-sediment data for Clark Fork at Turah Bridge, near Bonner, Montana, October 2002 through September 2003

[Abbreviations: $\mathrm{ft}^{3} / \mathrm{s}$, cubic feet per second; e, estimated; $\mathrm{mg} / \mathrm{L}$, milligrams per liter; ton/d, tons per day. Symbol: --, no data]

\begin{tabular}{|c|c|c|c|c|c|c|c|c|c|}
\hline \multirow[b]{2}{*}{ Day } & \multirow[b]{2}{*}{$\begin{array}{c}\text { Mean } \\
\text { streamflow } \\
\left(\mathrm{ft}^{3} / \mathrm{s}\right)\end{array}$} & \multicolumn{2}{|c|}{ Suspended sediment } & \multirow[b]{2}{*}{$\begin{array}{c}\text { Mean } \\
\text { streamflow } \\
\left(\mathrm{ft}^{3} / \mathrm{s}\right)\end{array}$} & \multicolumn{2}{|c|}{ Suspended sediment } & \multirow[b]{2}{*}{$\begin{array}{c}\text { Mean } \\
\text { streamflow } \\
\left(\mathrm{ft}^{3} / \mathrm{s}\right)\end{array}$} & \multicolumn{2}{|c|}{ Suspended sediment } \\
\hline & & $\begin{array}{l}\text { Mean } \\
\text { concen- } \\
\text { tration } \\
(\mathrm{mg} / \mathrm{L})\end{array}$ & $\begin{array}{c}\text { Discharge } \\
\text { (ton/d) }\end{array}$ & & $\begin{array}{c}\text { Mean } \\
\text { concen- } \\
\text { tration } \\
\text { (mg/L) }\end{array}$ & $\begin{array}{l}\text { Discharge } \\
\text { (ton/d) }\end{array}$ & & $\begin{array}{l}\text { Mean } \\
\text { concen- } \\
\text { tration } \\
(\mathrm{mg} / \mathrm{L})\end{array}$ & $\begin{array}{c}\text { Discharge } \\
\text { (ton/d) }\end{array}$ \\
\hline & & OCTOBER & & & NOVEMB & & & DECEMBE & \\
\hline 1 & 642 & 2 & 3.5 & e600 & 7 & 11 & 692 & 7 & 13 \\
\hline 2 & 639 & 3 & 5.2 & e600 & 7 & 11 & 691 & 7 & 13 \\
\hline 3 & 641 & 4 & 6.9 & e600 & 8 & 13 & 736 & 7 & 14 \\
\hline 4 & 661 & 4 & 7.1 & 649 & 8 & 14 & 722 & 7 & 14 \\
\hline 5 & 664 & 5 & 9.0 & 677 & 8 & 15 & 714 & 7 & 13 \\
\hline 6 & 669 & 6 & 11 & 702 & 9 & 17 & 705 & 7 & 13 \\
\hline 7 & 683 & 6 & 11 & 741 & 10 & 20 & 671 & 6 & 11 \\
\hline 8 & 674 & 6 & 11 & 768 & 10 & 21 & e600 & 6 & 9.7 \\
\hline 9 & 671 & 6 & 11 & 775 & 10 & 21 & 593 & 7 & 11 \\
\hline 10 & 698 & 6 & 11 & 764 & 10 & 21 & 582 & 8 & 13 \\
\hline 11 & 703 & 6 & 11 & 765 & 9 & 19 & 627 & 8 & 14 \\
\hline 12 & 717 & 6 & 12 & 749 & 9 & 18 & 654 & 8 & 14 \\
\hline 13 & 712 & 6 & 12 & 765 & 9 & 19 & 657 & 8 & 14 \\
\hline 14 & 715 & 6 & 12 & 779 & 9 & 19 & 720 & 9 & 17 \\
\hline 15 & 721 & 6 & 12 & 770 & 8 & 17 & 704 & 9 & 17 \\
\hline 16 & 721 & 7 & 14 & 756 & 8 & 16 & 700 & 8 & 15 \\
\hline 17 & 723 & 7 & 14 & 754 & 8 & 16 & 696 & 5 & 9.4 \\
\hline 18 & 725 & 7 & 14 & 746 & 8 & 16 & 638 & 3 & 5.2 \\
\hline 19 & 715 & 7 & 14 & 737 & 7 & 14 & e550 & 2 & 3.0 \\
\hline 20 & 708 & 6 & 11 & 736 & 7 & 14 & 541 & 3 & 4.4 \\
\hline 21 & 702 & 6 & 11 & 742 & 7 & 14 & 605 & 4 & 6.5 \\
\hline 22 & 699 & 6 & 11 & 748 & 7 & 14 & 614 & 6 & 9.9 \\
\hline 23 & 710 & 6 & 12 & 776 & 8 & 17 & 607 & 6 & 9.8 \\
\hline 24 & 715 & 6 & 12 & e700 & 9 & 17 & 593 & 5 & 8.0 \\
\hline 25 & 708 & 6 & 11 & e 600 & 9 & 15 & 535 & 4 & 5.8 \\
\hline 26 & 690 & 6 & 11 & 624 & 9 & 15 & 494 & 4 & 5.3 \\
\hline 27 & 707 & 6 & 11 & 658 & 8 & 14 & 558 & 6 & 9.0 \\
\hline 28 & 729 & 6 & 12 & 678 & 7 & 13 & 651 & 9 & 16 \\
\hline 29 & e750 & 7 & 14 & 703 & 7 & 13 & 657 & 9 & 16 \\
\hline 30 & e700 & 7 & 13 & 722 & 7 & 14 & 592 & 8 & 13 \\
\hline 31 & e650 & 7 & 12 & -- & -- & -- & 601 & 6 & 9.7 \\
\hline TOTAL & 21,562 & -- & 342.7 & 21,384 & -- & 478 & 19,700 & -- & 346.7 \\
\hline MEAN & 696 & 6 & 11 & 713 & 8 & 16 & 635 & 6 & 11 \\
\hline MAX & 750 & 7 & 14 & 779 & 10 & 21 & 736 & 9 & 17 \\
\hline MIN & 639 & 2 & 3.5 & 600 & 7 & 11 & 494 & 2 & 3.0 \\
\hline
\end{tabular}


TABLE 641

Table 6. Daily streamflow and suspended-sediment data for Clark Fork at Turah Bridge, near Bonner, Montana, October 2002 through September 2003-Continued

\begin{tabular}{|c|c|c|c|c|c|c|c|c|c|}
\hline \multirow[b]{2}{*}{ Day } & \multirow[b]{2}{*}{$\begin{array}{c}\text { Mean } \\
\text { streamflow } \\
\left(\mathrm{ft}^{3} / \mathrm{s}\right)\end{array}$} & \multicolumn{2}{|c|}{ Suspended sediment } & \multirow[b]{2}{*}{$\begin{array}{c}\text { Mean } \\
\text { streamflow } \\
\left(\mathrm{ft}^{3} / \mathrm{s}\right)\end{array}$} & \multicolumn{2}{|c|}{ Suspended sediment } & \multirow[b]{2}{*}{$\begin{array}{c}\text { Mean } \\
\text { streamflow } \\
\left(\mathrm{ft}^{3} / \mathrm{s}\right)\end{array}$} & \multicolumn{2}{|c|}{ Suspended sediment } \\
\hline & & $\begin{array}{c}\text { Mean } \\
\text { concen- } \\
\text { tration } \\
(\mathrm{mg} / \mathrm{L})\end{array}$ & $\begin{array}{l}\text { Discharge } \\
\text { (ton/d) }\end{array}$ & & $\begin{array}{c}\text { Mean } \\
\text { concen- } \\
\text { tration } \\
(\mathrm{mg} / \mathrm{L})\end{array}$ & $\begin{array}{c}\text { Discharge } \\
\text { (ton/d) }\end{array}$ & & $\begin{array}{l}\text { Mean } \\
\text { concen- } \\
\text { tration } \\
(\mathrm{mg} / \mathrm{L})\end{array}$ & $\begin{array}{c}\text { Discharge } \\
\text { (ton/d) }\end{array}$ \\
\hline & & JANUARY & & & FEBRUAR & & & MARCH & \\
\hline 3 & 651 & 5 & 8.8 & 1,240 & 39 & 131 & e650 & 6 & 11 \\
\hline 4 & 651 & 5 & 8.8 & 1,040 & 21 & 59 & e650 & 8 & 14 \\
\hline 5 & 663 & 5 & 9.0 & 943 & 17 & 43 & e650 & 5 & 8.8 \\
\hline 6 & 636 & 5 & 8.6 & e850 & 15 & 34 & 650 & 5 & 8.8 \\
\hline 10 & e500 & 4 & 5.4 & 792 & 11 & 24 & 749 & 10 & 20 \\
\hline 11 & $\mathrm{e} 450$ & 4 & 4.9 & 758 & 11 & 23 & 850 & 16 & 37 \\
\hline 12 & e500 & 3 & 4.0 & 713 & 11 & 21 & 1,150 & 75 & 233 \\
\hline 13 & 609 & 11 & 18 & 686 & 10 & 19 & 2,600 & 380 & 2,670 \\
\hline 14 & 711 & 16 & 31 & 723 & 10 & 20 & 4,620 & 790 & 9,850 \\
\hline 15 & 662 & 9 & 16 & 738 & 10 & 20 & 3,400 & 323 & 2,970 \\
\hline 16 & 609 & 5 & 8.2 & 718 & 12 & 23 & 2,410 & 141 & 917 \\
\hline 17 & e550 & 4 & 5.9 & 722 & 14 & 27 & 2,280 & 112 & 689 \\
\hline 18 & 490 & 4 & 5.3 & 708 & 11 & 21 & 1,780 & 64 & 308 \\
\hline 25 & 585 & 7 & 11 & e350 & 7 & 6.6 & 1,270 & 19 & 65 \\
\hline 26 & 680 & 10 & 18 & $\mathrm{e} 450$ & 7 & 8.5 & 1,220 & 16 & 53 \\
\hline 27 & 1,530 & 186 & 768 & e500 & 6 & 8.1 & 1,180 & 15 & 48 \\
\hline 28 & 1,840 & 230 & 1,140 & 578 & 7 & 11 & 1,120 & 14 & 42 \\
\hline 29 & 1,080 & 85 & 248 & -- & -- & -- & 1,090 & 14 & 41 \\
\hline 30 & 904 & 35 & 85 & -- & - & -- & 1,070 & 15 & 43 \\
\hline 31 & 1,070 & 72 & 208 & -- & -- & -- & 1,200 & 18 & 58 \\
\hline TOTAL & 21,257 & -- & $2,692.6$ & 22,633 & -- & $2,606.8$ & 42,216 & -- & $18,884.1$ \\
\hline MEAN & 686 & 25 & 87 & 808 & 23 & 93 & 1,362 & 73 & 609 \\
\hline MAX & 1,840 & 230 & 1,140 & 2,150 & 244 & 1,420 & 4,620 & 790 & 9,850 \\
\hline MIN & 400 & 3 & 4.0 & 350 & 6 & 6.6 & 643 & 4 & 7.0 \\
\hline
\end{tabular}


42 Water-Quality, Bed-Sediment, and Biological Data (October 2002 through September 2003) and Statistical Summaries of Data for Streams in the Upper Clark Fork Basin, Montana

Table 6. Daily streamflow and suspended-sediment data for Clark Fork at Turah Bridge, near Bonner, Montana, October 2002 through September 2003-Continued

\begin{tabular}{|c|c|c|c|c|c|c|c|c|c|}
\hline \multirow[b]{2}{*}{ Day } & \multirow[b]{2}{*}{$\begin{array}{c}\text { Mean } \\
\text { streamflow } \\
\left(\mathrm{ft}^{3} / \mathrm{s}\right)\end{array}$} & \multicolumn{2}{|c|}{ Suspended sediment } & \multirow[b]{2}{*}{$\begin{array}{c}\text { Mean } \\
\text { streamflow } \\
\left(\mathrm{ft}^{3} / \mathrm{s}\right)\end{array}$} & \multicolumn{2}{|c|}{ Suspended sediment } & \multirow[b]{2}{*}{$\begin{array}{c}\text { Mean } \\
\text { streamflow } \\
\left(\mathrm{ft}^{3} / \mathrm{s}\right)\end{array}$} & \multicolumn{2}{|c|}{ Suspended sediment } \\
\hline & & $\begin{array}{l}\text { Mean } \\
\text { concen- } \\
\text { tration } \\
(\mathrm{mg} / \mathrm{L})\end{array}$ & $\begin{array}{c}\text { Discharge } \\
\text { (ton/d) }\end{array}$ & & $\begin{array}{c}\text { Mean } \\
\text { concen- } \\
\text { tration } \\
(\mathrm{mg} / \mathrm{L})\end{array}$ & $\begin{array}{c}\text { Discharge } \\
\text { (ton/d) }\end{array}$ & & $\begin{array}{c}\text { Mean } \\
\text { concen- } \\
\text { tration } \\
(\mathrm{mg} / \mathrm{L})\end{array}$ & $\begin{array}{c}\text { Discharge } \\
\text { (ton/d) }\end{array}$ \\
\hline & & APRIL & & & MAY & & & JUNE & \\
\hline 3 & 1,600 & -- & -- & 2,420 & -- & -- & 5,210 & -- & -- \\
\hline 4 & 1,510 & -- & -- & 2,520 & -- & -- & 4,590 & -- & -- \\
\hline 5 & 1,410 & -- & -- & 2,930 & -- & - & 3,960 & -- & -- \\
\hline 6 & 1,360 & -- & -- & 2,940 & -- & -- & 3,560 & -- & -- \\
\hline 11 & 1,530 & -- & -- & 2,300 & -- & -- & 3,200 & -- & -- \\
\hline 12 & 1,720 & -- & -- & 2,270 & -- & -- & 2,990 & -- & -- \\
\hline 13 & 1,940 & -- & -- & 2,300 & -- & -- & 2,810 & -- & -- \\
\hline 14 & 2,180 & -- & - & 2,280 & -- & -- & 2,700 & -- & -- \\
\hline 15 & 2,220 & -- & -- & 2,350 & -- & -- & 2,610 & -- & -- \\
\hline 16 & 2,110 & -- & -- & 2,610 & .- & -- & 2,500 & -- & -- \\
\hline 17 & 1,950 & -- & -- & 2,770 & -- & -- & 2,370 & -- & -- \\
\hline 18 & 1,870 & -- & -- & 2,680 & -- & -- & 2,240 & -- & -- \\
\hline 25 & 2,730 & -- & -- & 3,790 & -- & -- & 1,850 & -- & -- \\
\hline 26 & 2,970 & -- & -- & 5,200 & -- & -- & 1,720 & -- & -- \\
\hline 27 & 2,730 & -- & -- & 5,830 & 205 & 3,230 & 1,600 & -- & -- \\
\hline 28 & 2,510 & -- & -- & 5,680 & 272 & 4,170 & 1,490 & -- & -- \\
\hline 29 & 2,340 & -- & -- & 5,910 & 311 & 4,960 & 1,430 & -- & -- \\
\hline 30 & 2,370 & -- & -- & 6,590 & 300 & 5,340 & 1,360 & - & -. \\
\hline 31 & -- & -- & -- & 6,790 & 276 & 5,060 & -- & -- & -- \\
\hline TOTAL & 56,760 & -- & -- & 99,670 & -- & -- & 86,140 & -- & -- \\
\hline MEAN & 1,892 & -- & -. & 3,215 & -- & -- & 2,871 & -- & -- \\
\hline MAX & 2,970 & -- & -- & 6,790 & -- & -- & 6,430 & -- & -- \\
\hline MIN & 1,250 & -- & -- & 2,130 & -- & -- & 1,360 & -- & -- \\
\hline
\end{tabular}


TABLE $6 \quad 43$

Table 6. Daily streamflow and suspended-sediment data for Clark Fork at Turah Bridge, near Bonner, Montana, October 2002 through September 2003-Continued

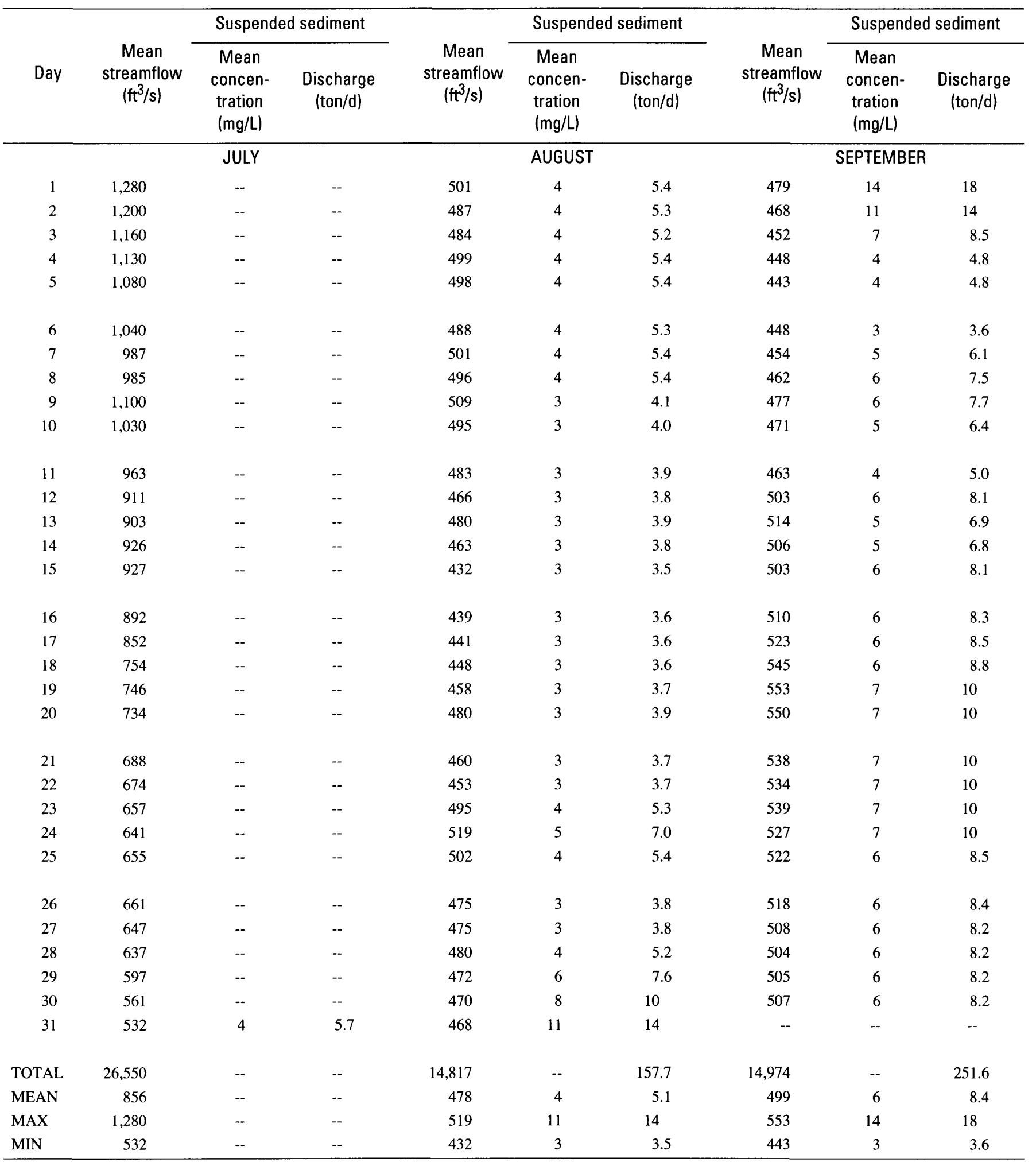

TOTAL FOR WATER YEAR 2003:

STREAMFLOW--447,663 $\mathrm{ft}^{3} / \mathrm{s}$

SEDIMENT LOAD--54,735.9 tons (partial year) 

in the Upper Clark Fork Basin, Montana

Table 7. Daily streamflow and suspended-sediment data for Clark Fork above Missoula, Montana, 0 ctober 2002 through September 2003

[Abbreviations: $\mathrm{ft}^{3} / \mathrm{s}$, cubic feet per second; e, estimated; $\mathrm{mg} / \mathrm{L}$, milligrams per liter; ton/d, tons per day. Symbol: --, no data]

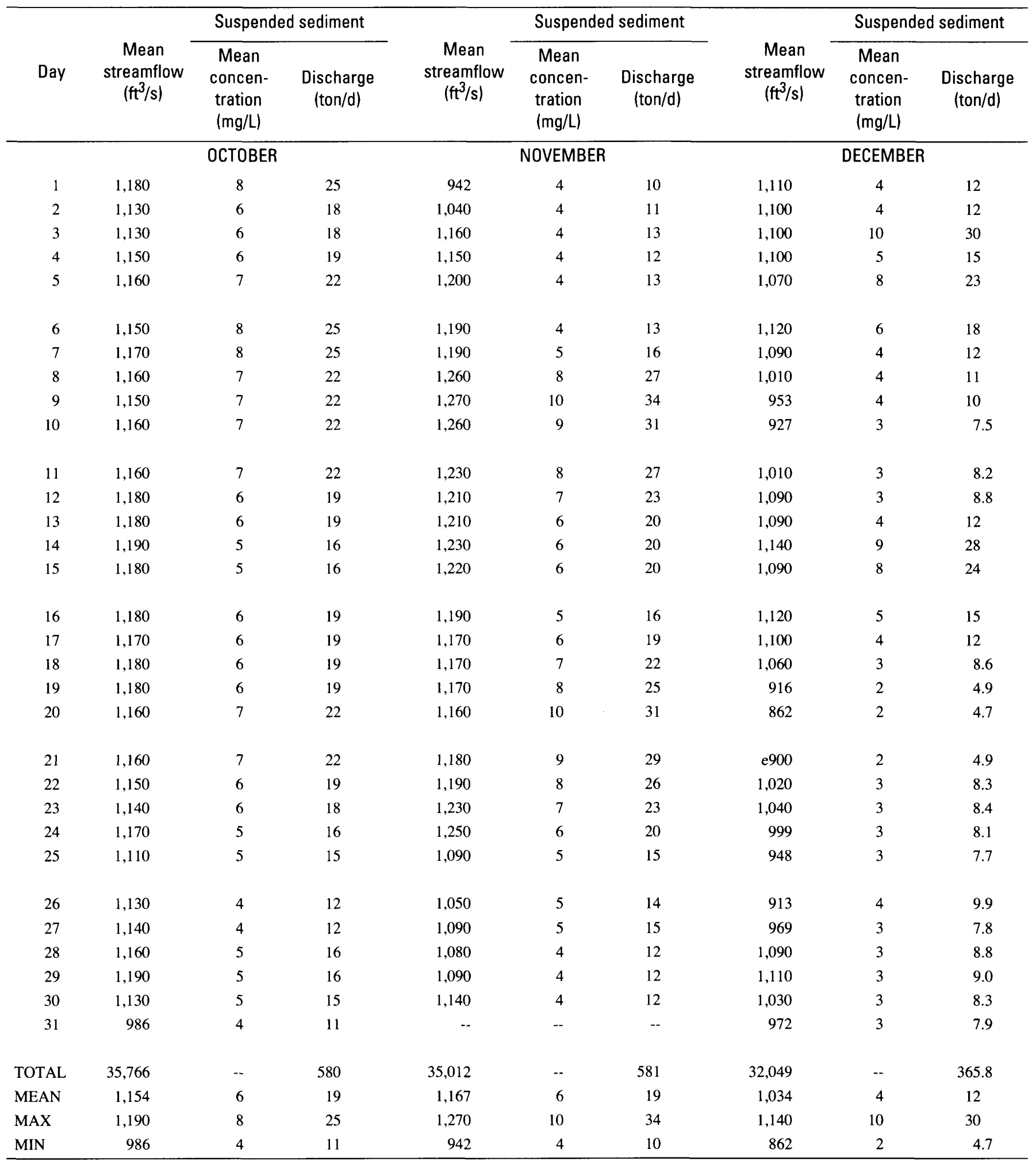


TABLE 745

Table 7. Daily streamflow and suspended-sediment data for Clark Fork above Missoula, Montana October 2002 through September 2003-Continued

\begin{tabular}{|c|c|c|c|c|c|c|c|c|c|}
\hline Day & $\begin{array}{c}\text { Mean } \\
\text { streamflow } \\
\left(\mathrm{ft}^{3} / \mathrm{s}\right)\end{array}$ & \multicolumn{2}{|c|}{ Suspended sediment } & $\begin{array}{c}\text { Mean } \\
\text { streamflow } \\
\left(\mathrm{ft}^{3} / \mathrm{s}\right)\end{array}$ & \multicolumn{2}{|c|}{ Suspended sediment } & $\begin{array}{c}\text { Mean } \\
\text { streamflow } \\
\left(\mathrm{ft}^{3} / \mathrm{s}\right)\end{array}$ & \multicolumn{2}{|c|}{ Suspended sediment } \\
\hline & & JANUARY & & & FEBRUAR & & & MARCH & \\
\hline 3 & 1,060 & 3 & 8.6 & 2,200 & 26 & 154 & 1,100 & 4 & 12 \\
\hline 4 & 1,080 & 3 & 8.7 & 1,760 & 16 & 76 & 1,060 & 4 & 11 \\
\hline 5 & 1,070 & 3 & 8.7 & 1,580 & 11 & 47 & 1,050 & 3 & 8.5 \\
\hline 6 & 1,060 & 3 & 8.6 & 1,430 & 9 & 35 & 1,080 & 5 & 15 \\
\hline 11 & e750 & 3 & 6.1 & 1,200 & 5 & 16 & 1,390 & 9 & 34 \\
\hline 12 & 877 & 4 & 9.5 & 1,160 & 5 & 16 & 1,680 & 20 & 91 \\
\hline 13 & 1,070 & 5 & 14 & 1,130 & 5 & 15 & 3,430 & 188 & 1,740 \\
\hline 14 & 1,190 & 5 & 16 & 1,160 & 6 & 19 & 5,740 & 372 & 5,770 \\
\hline 15 & 1,110 & 4 & 12 & 1,190 & 7 & 22 & 5,170 & 160 & 2,230 \\
\hline 16 & 1,040 & 3 & 8.4 & 1,160 & 8 & 25 & 3,900 & 82 & 863 \\
\hline 17 & 859 & 2 & 4.6 & 1,170 & 9 & 28 & 3,940 & 63 & 670 \\
\hline 18 & 797 & 3 & 6.5 & 1,160 & 9 & 28 & 3,180 & 53 & 455 \\
\hline 25 & 1,020 & 11 & 30 & 677 & 3 & 5.5 & 2,430 & 19 & 125 \\
\hline 26 & 1,180 & 15 & 48 & 846 & 3 & 6.9 & 2,310 & 16 & 100 \\
\hline 27 & 1,820 & 66 & 324 & 946 & 4 & 10 & 2,230 & 15 & 90 \\
\hline 28 & 2,550 & 170 & 1,170 & 1,050 & 5 & 14 & 2,110 & 14 & 80 \\
\hline 29 & 1,530 & 47 & 194 & -- & -- & -. & 2,000 & 14 & 76 \\
\hline 30 & 1,420 & 19 & 73 & -- & -- & -- & 2,000 & 15 & 81 \\
\hline 31 & 1,610 & 20 & 87 & -- & - & -- & 2,200 & 16 & 95 \\
\hline TOTAL & 33,721 & -. & $2,287.2$ & 37,658 & -- & $2,823.7$ & 69,460 & -- & $13,651.5$ \\
\hline MEAN & 1,088 & 16 & 74 & 1,345 & 16 & 101 & 2,241 & 40 & 440 \\
\hline MAX & 2,550 & 170 & 1,170 & 3,130 & 161 & 1,360 & 5,740 & 372 & 5,770 \\
\hline MIN & 700 & 2 & 4.6 & 677 & 3 & 5.5 & 1,000 & 3 & 8.1 \\
\hline
\end{tabular}


Water-Quality, Bed-Sediment, and Biological Data (October 2002 through September 2003) and Statistical Summaries of Data for Streams in the Upper Clark Fork Basin, Montana

Table 7. Daily streamflow and suspended-sediment data for Clark Fork above Missoula, Montana, 0ctober 2002 through September 2003-Continued

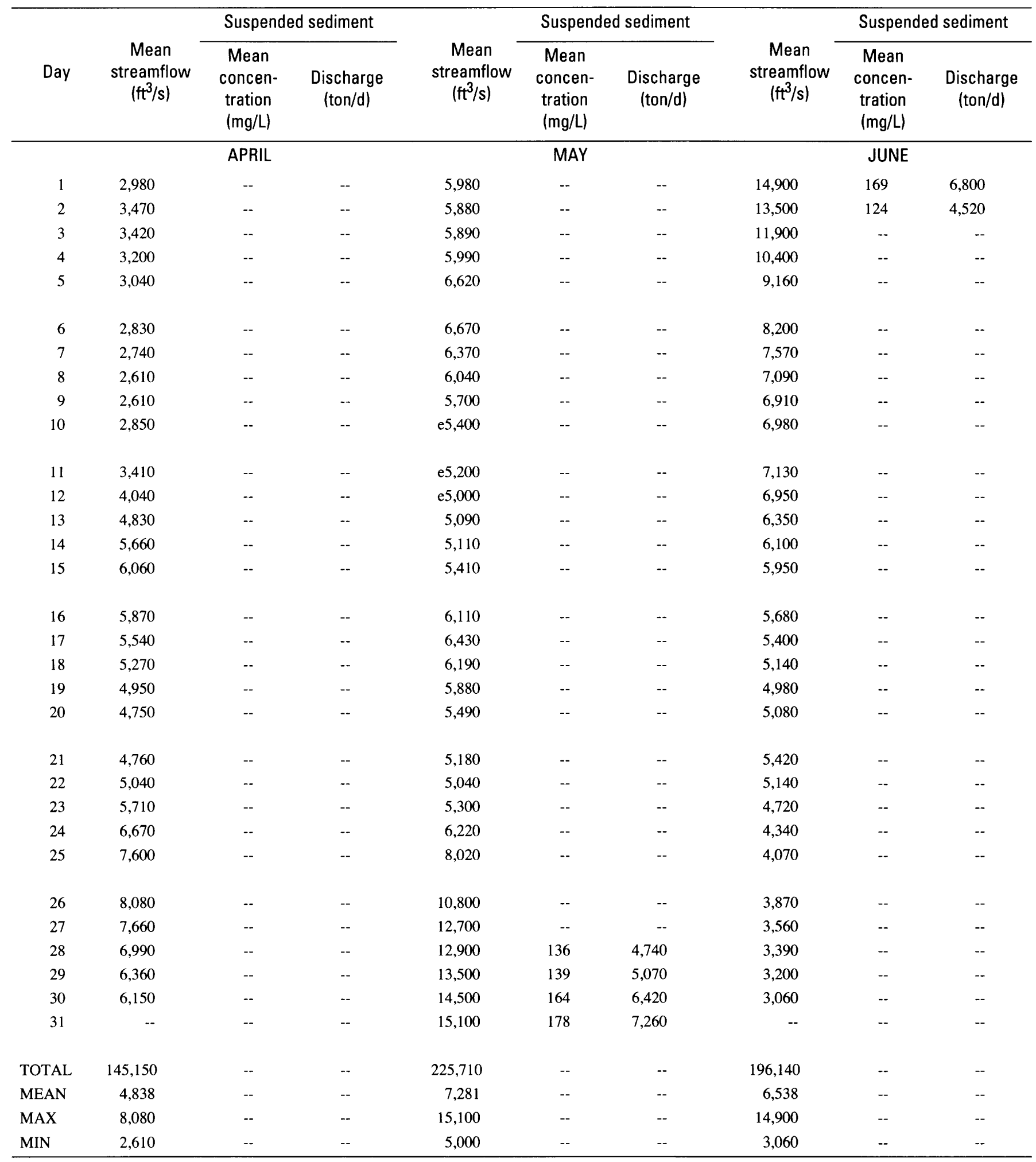


Table 7. Daily streamflow and suspended-sediment data for Clark Fork above Missoula, Montana, October 2002 through September 2003-Continued

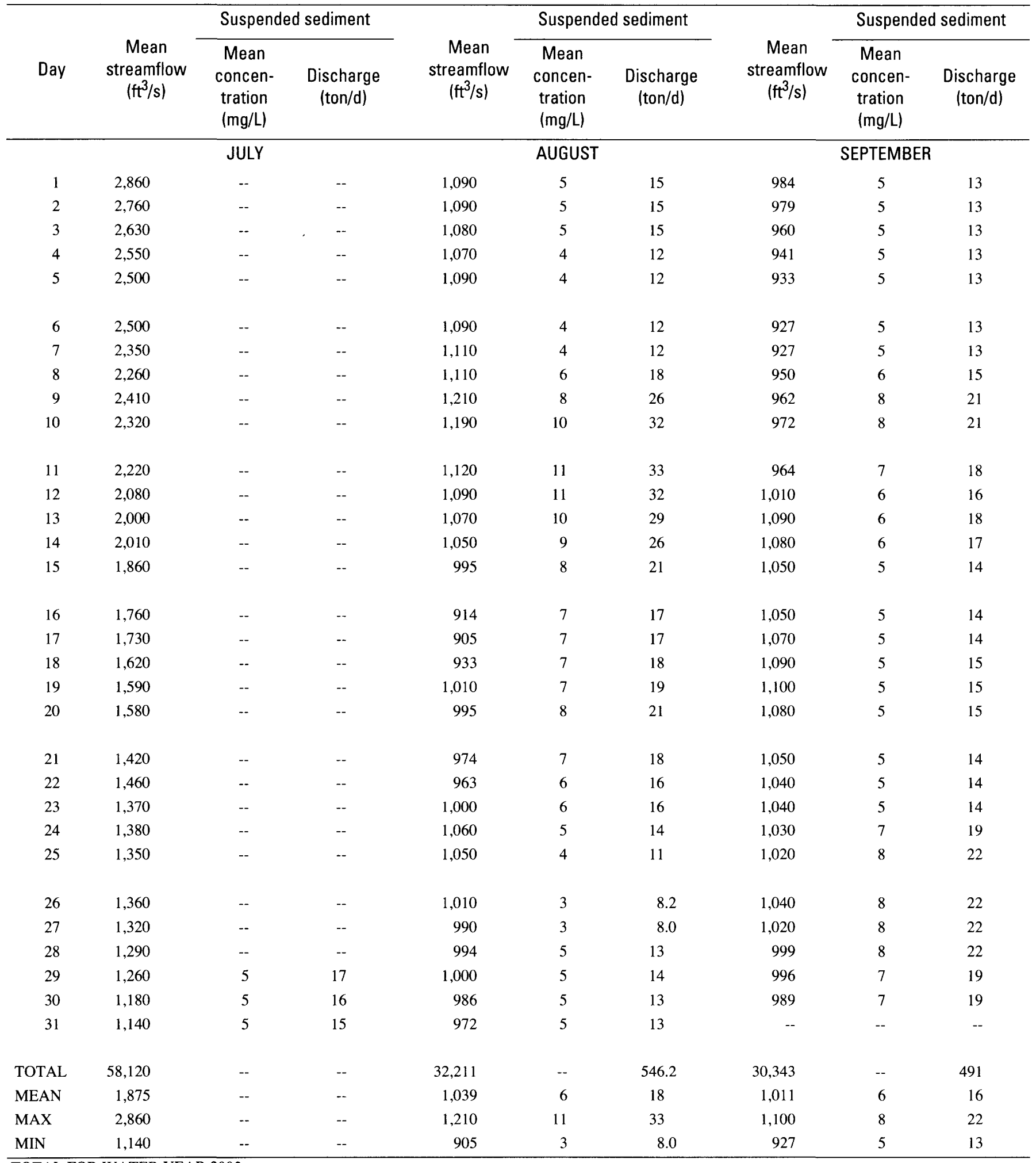

TOTAL FOR WATER YEAR 2003:

STREAMFLOW--931,340 $\mathrm{ft}^{3} / \mathrm{s}$

SEDIMENT LOAD--56,184.4 tons (partial year) 

in the upper Clark Fork Basin, Montana

Table 8. Chemical and suspended-sediment analyses of field replicates for water samples, upper Clark Fork basin, Montana

[The terms "filtered" and "unfiltered recoverable" replace the former terms "dissolved" and "total recoverable," respectively. Abbreviations: E, estimated; $\mu \mathrm{g} / \mathrm{L}$, micrograms per liter; $\mathrm{mg} / \mathrm{L}$, milligrams per liter; $\mathrm{mm}$, millimeter. Symbol: <, less than analytical detection limit]

\begin{tabular}{|c|c|c|c|c|c|c|c|c|c|c|}
\hline $\begin{array}{l}\text { Station } \\
\text { number }\end{array}$ & \multicolumn{3}{|c|}{ Station name } & Date & Time & $\begin{array}{c}\text { Hard- } \\
\text { ness, } \\
\text { filtered } \\
\text { (mg/L as } \\
\mathrm{CaCO}_{3} \text { ) }\end{array}$ & $\begin{array}{l}\text { Calcium, } \\
\text { filtered } \\
\text { (mg/L) }\end{array}$ & $\begin{array}{l}\text { Magne- } \\
\text { sium, } \\
\text { filtered } \\
\text { (mg/L) }\end{array}$ & $\begin{array}{c}\text { Arsenic, } \\
\text { filtered } \\
(\mu \mathrm{g} / \mathrm{L})\end{array}$ & $\begin{array}{c}\text { Arsenic, } \\
\text { unfiltered } \\
\text { recov- } \\
\text { erable } \\
(\mu \mathrm{g} / \mathrm{L})\end{array}$ \\
\hline \multirow[t]{2}{*}{12324200} & \multicolumn{3}{|c|}{ Clark Fork at Deer Lodge } & $03-18-03$ & 1215 & 220 & 64.6 & 13.5 & 16.2 & 27 \\
\hline & \multicolumn{3}{|c|}{ Clark Fork at Deer Lodge } & $03-18-03$ & 1220 & 220 & 64.9 & 13.8 & 16.3 & 27 \\
\hline \multirow[t]{2}{*}{12323230} & \multirow{2}{*}{\multicolumn{3}{|c|}{$\begin{array}{l}\text { Blacktail Creek at Harrison Avenue, at Butte } \\
\text { Blacktail Creek at Harrison Avenue, at Butte }\end{array}$}} & $04-02-03$ & 0850 & 77 & 21.4 & 5.66 & 4.4 & 6 \\
\hline & & & & $04-02-03$ & 0855 & 78 & 21.6 & 5.72 & 4.3 & 6 \\
\hline \multirow[t]{2}{*}{12323750} & \multirow{2}{*}{\multicolumn{3}{|c|}{$\begin{array}{l}\text { Silver Bow Creek at Warm Springs } \\
\text { Silver Bow Creek at Warm Springs }\end{array}$}} & $04-02-03$ & 1435 & 200 & 58.6 & 12.3 & 15.8 & 19 \\
\hline & & & & $04-02-03$ & 1440 & 200 & 59.2 & 12.3 & 16.1 & 20 \\
\hline \multirow[t]{2}{*}{12334550} & \multirow{2}{*}{\multicolumn{3}{|c|}{$\begin{array}{l}\text { Clark Fork at Turah Bridge, near Bonner } \\
\text { Clark Fork at Turah Bridge, near Bonner }\end{array}$}} & $04-29-03$ & 1140 & 100 & 29.1 & 7.42 & 4.8 & 7 \\
\hline & & & & $04-29-03$ & 1145 & 100 & 29.1 & 7.40 & 4.9 & 7 \\
\hline \multirow[t]{2}{*}{12340500} & \multirow{2}{*}{\multicolumn{3}{|c|}{$\begin{array}{l}\text { Clark Fork above Missoula } \\
\text { Clark Fork above Missoula }\end{array}$}} & $05-28-03$ & 0910 & 71 & 19.4 & 5.34 & 3.4 & 8 \\
\hline & & & & $05-28-03$ & 0915 & 72 & 19.9 & 5.47 & 3.4 & 8 \\
\hline \multirow[t]{2}{*}{12323600} & \multirow{2}{*}{\multicolumn{3}{|c|}{$\begin{array}{l}\text { Silver Bow Creek at Opportunity } \\
\text { Silver Bow Creek at Opportunity }\end{array}$}} & 06-03-03 & 1430 & 130 & 40.5 & 7.25 & 9.5 & 14 \\
\hline & & & & 06-03-03 & 1435 & 130 & 40.6 & 7.27 & 9.9 & 13 \\
\hline \multirow[t]{2}{*}{12331800} & \multirow{2}{*}{\multicolumn{3}{|c|}{$\begin{array}{l}\text { Clark Fork near Drummond } \\
\text { Clark Fork near Drummond }\end{array}$}} & 08-26-03 & 1055 & 280 & 77.9 & 19.7 & 11.4 & 14 \\
\hline & & & & $08-26-03$ & 1100 & 280 & 79.7 & 20.1 & 11.6 & 14 \\
\hline $\begin{array}{l}\text { Station } \\
\text { number }\end{array}$ & Date & $\begin{array}{l}\text { Cadmium, } \\
\text { filtered } \\
(\mu \mathrm{g} / \mathrm{L})\end{array}$ & $\begin{array}{c}\text { Cadmium, } \\
\text { unfiltered } \\
\text { recoverable } \\
(\mu \mathrm{g} / \mathrm{L})\end{array}$ & \multicolumn{2}{|c|}{$\begin{array}{c}\text { Copper, } \\
\text { filtered } \\
(\mu g / L)\end{array}$} & $\begin{array}{c}\text { Copper, } \\
\text { unfiltered } \\
\text { recoverable } \\
(\mu \mathrm{g} / \mathrm{L})\end{array}$ & $\begin{array}{l}\text { Iron, } \\
\text { filtered } \\
(\mu \mathrm{g} / \mathrm{L})\end{array}$ & \multicolumn{2}{|c|}{$\begin{array}{c}\text { Iron, } \\
\text { unfiltered } \\
\text { recov- } \\
\text { erable } \\
(\mu \mathrm{g} / \mathrm{L})\end{array}$} & $\begin{array}{c}\text { Lead, } \\
\text { filtered } \\
(\mu \mathrm{g} / \mathrm{L})\end{array}$ \\
\hline \multirow[t]{2}{*}{12324200} & $03-18-03$ & 0.12 & 0.41 & \multicolumn{2}{|c|}{14.0} & 95.8 & 12 & \multirow{2}{*}{\multicolumn{2}{|c|}{$\begin{array}{l}1,420 \\
1,370\end{array}$}} & 0.25 \\
\hline & $03-18-03$ & .12 & .42 & 14 & & 89.6 & 13 & & & .25 \\
\hline \multirow[t]{2}{*}{12323230} & $04-02-03$ & .10 & .11 & \multicolumn{2}{|c|}{6.8} & 16.9 & 312 & 1,4 & & .62 \\
\hline & $04-02-03$ & .06 & .10 & 6. & & 16.5 & 314 & 1,4 & & .56 \\
\hline 12323750 & $04-02-03$ & .04 & .11 & 7. & & 18.4 & 15 & & & .14 \\
\hline & $04-02-03$ & .04 & .12 & 7. & & 18.4 & 16 & & & .15 \\
\hline 12334550 & $04-29-03$ & E.03 & .12 & 3. & & 17.6 & 29 & & & .10 \\
\hline & $04-29-03$ & E.03 & .13 & 3. & & 17.7 & 26 & & & .10 \\
\hline 12340500 & $05-28-03$ & E.02 & .27 & 3. & & 39.8 & 40 & 1,6 & & .17 \\
\hline & $05-28-03$ & E.02 & .29 & 3. & & 40.6 & 38 & 1,7 & & .18 \\
\hline 12323600 & $06-03-03$ & .29 & .58 & 25 . & & 60.6 & 38 & & & .27 \\
\hline & $06-03-03$ & .30 & .57 & 25 . & & 60.6 & 40 & & & .28 \\
\hline 12331800 & $08-26-03$ & E.03 & .08 & 3. & & 9.4 & $<8$ & & & $<.08$ \\
\hline & $08-26-03$ & E. 02 & .07 & 3. & & 9.1 & $<8$ & & & E.05 \\
\hline
\end{tabular}


Table 8. Chemical and suspended-sediment analyses of field replicates for water samples, upper Clark Fork basin, Montana-Continued

\begin{tabular}{|c|c|c|c|c|c|c|c|c|}
\hline $\begin{array}{l}\text { Station } \\
\text { number }\end{array}$ & Date & $\begin{array}{c}\text { Lead, } \\
\text { unfiltered } \\
\text { recoverable } \\
(\mu \mathrm{g} / \mathrm{L})\end{array}$ & $\begin{array}{c}\text { Manganese, } \\
\text { filtered } \\
(\mu \mathrm{g} / \mathrm{L})\end{array}$ & $\begin{array}{c}\text { Manganese, } \\
\text { unfiltered } \\
\text { recoverable } \\
(\mu \mathrm{g} / \mathrm{L})\end{array}$ & $\begin{array}{c}\text { Zinc, } \\
\text { filtered } \\
(\mu \mathrm{g} / \mathrm{L})\end{array}$ & $\begin{array}{c}\text { Zinc, } \\
\text { unfiltered } \\
\text { recoverable } \\
(\mu \mathrm{g} / \mathrm{L})\end{array}$ & $\begin{array}{l}\text { Sediment, } \\
\text { suspended, } \\
\text { diameter, } \\
\text { percent } \\
\text { finer than } \\
0.062 \mathrm{~mm}\end{array}$ & $\begin{array}{c}\text { Sediment, } \\
\text { suspended } \\
(\mathrm{mg} / \mathrm{L})\end{array}$ \\
\hline \multirow[t]{2}{*}{12324200} & $03-18-03$ & 22.1 & 83.9 & 259 & 19 & 102 & 68 & 60 \\
\hline & $03-18-03$ & 20.9 & 87.0 & 253 & 20 & 97 & 65 & 64 \\
\hline \multirow[t]{2}{*}{12323230} & $04-02-03$ & 4.86 & 39.7 & 72 & 8 & 27 & 82 & 31 \\
\hline & $04-02-03$ & 4.76 & 39.9 & 71 & 7 & 26 & 86 & 35 \\
\hline \multirow[t]{2}{*}{12323750} & $04-02-03$ & 5.13 & 122 & 172 & 7 & 32 & 92 & 7 \\
\hline & $04-02-03$ & 5.05 & 122 & 151 & 7 & 32 & 89 & 8 \\
\hline \multirow[t]{2}{*}{12334550} & $04-29-03$ & 3.19 & 6.4 & 58 & 2 & 27 & 68 & 31 \\
\hline & $04-29-03$ & 2.97 & 6.2 & 58 & 2 & 26 & 67 & 29 \\
\hline \multirow[t]{2}{*}{12340500} & $05-28-03$ & 7.50 & 13.2 & 143 & 1 & 77 & 75 & 137 \\
\hline & $05-28-03$ & 7.45 & 12.9 & 146 & 1 & 74 & 75 & 135 \\
\hline \multirow[t]{2}{*}{12323600} & $06-03-03$ & 10.2 & 172 & 211 & 40 & 119 & 84 & 17 \\
\hline & 06-03-03 & 10.3 & 177 & 211 & 40 & 119 & 83 & 17 \\
\hline \multirow[t]{2}{*}{12331800} & $08-26-03$ & .98 & 10.4 & 79 & 3 & 12 & 58 & 18 \\
\hline & $08-26-03$ & .93 & 10.3 & 77 & 3 & 12 & 51 & 23 \\
\hline
\end{tabular}


50 Water-Quality, Bed-Sediment, and Biological Data (October 2002 through September 2003) and Statistical Summaries of Data for Streams in the Upper Clark Fork Basin, Montana

Table 9. Precision of chemical and suspended-sediment analyses of field replicates for water samples, upper Clark Fork basin, Montana

[The terms "filtered" and "unfiltered recoverable" replace the former terms "dissolved" and "total recoverable," respectively. Abbreviations: $\mu \mathrm{g} / \mathrm{L}$, micrograms per liter; $\mathrm{mg} / \mathrm{L}$, milligrams per liter; $\mathrm{mm}$, millimeter]

\begin{tabular}{lccc}
\hline \multicolumn{1}{c}{ Constituent and reporting unit } & $\begin{array}{c}\text { Number of } \\
\text { replicate pairs }\end{array}$ & $\begin{array}{c}\text { Standard } \\
\text { deviation, } \\
\text { in listed } \\
\text { units }\end{array}$ & $\begin{array}{c}\text { Relative } \\
\text { standard } \\
\text { deviation, } \\
\text { in percent }\end{array}$ \\
\hline Calcium, filtered, $\mathrm{mg} / \mathrm{L}$ & 7 & 0.53 & 1.2 \\
Magnesium, filtered, $\mathrm{mg} / \mathrm{L}$ & 7 & .14 & 1.4 \\
Arsenic, unfiltered recoverable, $\mu \mathrm{g} / \mathrm{L}$ & 7 & .38 & 2.8 \\
Arsenic, filtered, $\mu \mathrm{g} / \mathrm{L}$ & 7 & .15 & 1.6 \\
Cadmium, unfiltered recoverable, $\mu \mathrm{g} / \mathrm{L}$ & 7 & .01 & 3.5 \\
Cadmium, filtered, $\mu \mathrm{g} / \mathrm{L}$ & 7 & .01 & 13 \\
Copper, unfiltered recoverable, $\mu \mathrm{g} / \mathrm{L}$ & 7 & 1.7 & 4.6 \\
Copper, filtered, $\mu \mathrm{g} / \mathrm{L}$ & 7 & .13 & 1.4 \\
Iron, unfiltered recoverable, $\mu \mathrm{g} / \mathrm{L}$ & 7 & 19 & 2.2 \\
Iron, filtered, $\mu \mathrm{g} / \mathrm{L}$ & 7 & 1.3 & 2.0 \\
Lead, unfiltered recoverable, $\mu \mathrm{g} / \mathrm{L}$ & 7 & .33 & 4.3 \\
Lead, filtered, $\mu \mathrm{g} / \mathrm{L}$ & 7 & .02 & 7.5 \\
Manganese, unfiltered recoverable, $\mu \mathrm{g} / \mathrm{L}$ & 7 & 5.9 & 4.2 \\
Manganese, filtered, $\mu \mathrm{g} / \mathrm{L}$ & 7 & 1.6 & 2.4 \\
Zinc, unfiltered recoverable, $\mu \mathrm{g} / \mathrm{L}$ & 7 & 1.6 & 2.9 \\
Zinc, filtered, $\mu \mathrm{g} / \mathrm{L}$ & 7 & .38 & 3.3 \\
Sediment, suspended, $\mathrm{mg} / \mathrm{L}$ & 7 & 2.2 & 5.0 \\
Sediment, suspended, percent finer than $0.062 \mathrm{~mm}$ & 7 & 2.5 & 3.3 \\
\hline
\end{tabular}


Table 10. Precision of chemical analyses of laboratory replicates for water samples, upper Clark Fork basin, Montana

[The terms "filtered" and "unfiltered recoverable" replace the former terms "dissolved" and "total recoverable," respectively. Abbreviations: $\mu \mathrm{g} / \mathrm{L}$, micrograms per liter; $\mathrm{mg} / \mathrm{L}$, milligrams per liter]

\begin{tabular}{lcccc}
\hline \multicolumn{1}{c}{ Constituent and reporting unit } & $\begin{array}{c}\text { Number of } \\
\text { replicate pairs }\end{array}$ & $\begin{array}{c}\text { Standard } \\
\text { deviation, } \\
\text { in listed } \\
\text { units }\end{array}$ & $\begin{array}{c}\text { Relative } \\
\text { standard } \\
\text { deviation, } \\
\text { in percent }\end{array}$ & $\begin{array}{c}\text { Within limits of } \\
\text { data-quality } \\
\text { objective }\end{array}$ \\
\hline Calcium, filtered, $\mathrm{mg} / \mathrm{L}$ & 10 & 0.93 & 2.1 & Yes \\
Magnesium, filtered, $\mathrm{mg} / \mathrm{L}$ & 10 & .17 & 1.6 & Yes \\
Arsenic, unfiltered recoverable, $\mu \mathrm{g} / \mathrm{L}$ & 10 & .67 & 4.3 & Yes \\
Arsenic, filtered, $\mu \mathrm{g} / \mathrm{L}$ & 10 & .16 & 1.1 & Yes \\
Cadmium, unfiltered recoverable, $\mu \mathrm{g} / \mathrm{L}$ & 10 & .01 & 4.8 & Yes \\
Cadmium, filtered, $\mu \mathrm{g} / \mathrm{L}$ & 10 & .00 & 5.0 & Yes \\
Copper, unfiltered recoverable, $\mu \mathrm{g} / \mathrm{L}$ & 10 & .56 & 2.8 & Yes \\
Copper, filtered, $\mu \mathrm{g} / \mathrm{L}$ & 10 & .08 & 1.5 & Yes \\
Iron, unfiltered recoverable, $\mu \mathrm{g} / \mathrm{L}$ & 10 & 5.1 & 1.1 & Yes \\
Iron, filtered, $\mu \mathrm{g} / \mathrm{L}$ & 10 & 1.6 & 5.8 & Yes \\
Lead, unfiltered recoverable, $\mu \mathrm{g} / \mathrm{L}$ & 10 & .06 & 2.2 & Yes \\
Lead, filtered, $\mu \mathrm{g} / \mathrm{L}$ & 10 & .02 & 11 & Yes \\
Manganese,, $\mathrm{nnfiltered}$ recoverable, $\mu \mathrm{g} / \mathrm{L}$ & 10 & .84 & 1.4 & Yes \\
Manganese, filtered, $\mu \mathrm{g} / \mathrm{L}$ & 10 & 1.9 & 9.4 & Yes \\
Zinc, unfiltered recoverable, $\mu \mathrm{g} / \mathrm{L}$ & 10 & .42 & 1.3 & Yes \\
Zinc, filtered, $\mu \mathrm{g} / \mathrm{L}$ & 10 & .24 & 1.7 & Yes \\
\hline
\end{tabular}

\footnotetext{
${ }^{1}$ Statistics calculated using laboratory reporting level for censored values less than the detection capability of the instrument.
} 
52 Water-Quality, Bed-Sediment, and Biological Data (October 2002 through September 2003) and Statistical Summaries of Data for Streams in the Upper Clark Fork Basin, Montana

Table 11. Recovery efficiency for trace-element analyses of laboratory-spiked deionized-water blanks

[The terms "filtered" and "unfiltered recoverable" replace the former terms "dissolved" and "total recoverable," respectively. Abbreviation: $\mu \mathrm{g} / \mathrm{L}$, micrograms per liter]

\begin{tabular}{lcccc}
\hline \multicolumn{1}{c}{ Constituent and reporting unit } & $\begin{array}{c}\text { Number of } \\
\text { samples }\end{array}$ & $\begin{array}{c}\text { 95-percent } \\
\text { confidence } \\
\text { interval for spike } \\
\text { recovery, } \\
\text { in percent }\end{array}$ & $\begin{array}{c}\text { Mean spike } \\
\text { recovery, } \\
\text { in percent }\end{array}$ & $\begin{array}{c}\text { Within limits of } \\
\text { data-quality } \\
\text { objective }\end{array}$ \\
\hline Arsenic, unfiltered recoverable, $\mu \mathrm{g} / \mathrm{L}$ & 4 & $78.5-115$ & 96.6 & Yes \\
Arsenic, filtered, $\mu \mathrm{g} / \mathrm{L}$ & 4 & $71.3-104$ & 87.9 & Yes \\
Cadmium, unfiltered recoverable, $\mu \mathrm{g} / \mathrm{L}$ & 4 & $94.1-101$ & 97.5 & Yes \\
Cadmium, filtered, $\mu \mathrm{g} / \mathrm{L}$ & 4 & $92.6-105$ & 98.6 & Yes \\
Copper, unfiltered recoverable, $\mu \mathrm{g} / \mathrm{L}$ & 3 & $93.2-102$ & 97.6 & Yes \\
Copper, filtered, $\mu \mathrm{g} / \mathrm{L}$ & 4 & $93.0-108$ & 100.4 & Yes \\
Iron, unfiltered recoverable, $\mu \mathrm{g} / \mathrm{L}$ & 4 & $96.4-107$ & 101.6 & Yes \\
Iron, filtered, $\mu \mathrm{g} / \mathrm{L}$ & 4 & $87.4-98.8$ & 93.1 & Yes \\
Lead, unfiltered recoverable, $\mu \mathrm{g} / \mathrm{L}$ & 4 & $93.9-98.1$ & 96.0 & Yes \\
Lead, filtered, $\mu \mathrm{g} / \mathrm{L}$ & 3 & $92.3-102$ & 97.2 & Yes \\
Manganese, unfiltered recoverable, $\mu \mathrm{g} / \mathrm{L}$ & 4 & $90.7-101$ & 95.8 & Yes \\
Manganese, filtered, $\mu \mathrm{g} / \mathrm{L}$ & 4 & $79.7-114$ & 96.6 & Yes \\
Zinc, unfiltered recoverable, $\mu \mathrm{g} / \mathrm{L}$ & 4 & $93.2-105$ & 99.1 & Yes \\
Zinc, filtered, $\mu \mathrm{g} / \mathrm{L}$ & 4 & $89.8-113$ & 101.4 & Yes \\
\hline
\end{tabular}


TABLE 1253

Table 12. Recovery efficiency for trace-element analyses of laboratory-spiked stream samples, upper Clark Fork basin, Montana

[The terms "filtered" and "unfiltered recoverable" replace the former terms "dissolved" and "total recoverable," respectively. Abbreviation: $\mu \mathrm{g} / \mathrm{L}$, micrograms per liter]

\begin{tabular}{|c|c|c|c|c|}
\hline Constituent and reporting unit & $\begin{array}{c}\text { Number of } \\
\text { samples }\end{array}$ & $\begin{array}{c}\text { 95-percent } \\
\text { confidence } \\
\text { interval for } \\
\text { spike } \\
\text { recovery, } \\
\text { in percent }\end{array}$ & $\begin{array}{c}\text { Mean spike } \\
\text { recovery, } \\
\text { in percent }\end{array}$ & $\begin{array}{c}\text { Within limits of } \\
\text { data-quality } \\
\text { objective }\end{array}$ \\
\hline Arsenic, unfiltered recoverable, $\mu \mathrm{g} / \mathrm{L}$ & 4 & $100-117$ & 108.6 & Yes \\
\hline Arsenic, filtered, $\mu \mathrm{g} / \mathrm{L}$ & 4 & $80.2-109$ & 94.6 & Yes \\
\hline Cadmium, unfiltered recoverable, $\mu \mathrm{g} / \mathrm{L}$ & 4 & $94.4-104$ & 99.0 & Yes \\
\hline Cadmium, filtered, $\mu \mathrm{g} / \mathrm{L}$ & 4 & $91.4-110$ & 100.5 & Yes \\
\hline Copper, unfiltered recoverable, $\mu \mathrm{g} / \mathrm{L}$ & 4 & $89.7-93.5$ & 91.6 & Yes \\
\hline Copper, filtered, $\mu \mathrm{g} / \mathrm{L}$ & 4 & 88.9-103 & 95.8 & Yes \\
\hline Iron, unfiltered recoverable, $\mu \mathrm{g} / \mathrm{L}$ & 4 & $100-113$ & 106.4 & Yes \\
\hline Iron, filtered, $\mu \mathrm{g} / \mathrm{L}$ & 4 & $91.6-102$ & 96.7 & Yes \\
\hline Lead, unfiltered recoverable, $\mu \mathrm{g} / \mathrm{L}$ & 4 & $93.7-99.9$ & 96.8 & Yes \\
\hline Lead, filtered, $\mu \mathrm{g} / \mathrm{L}$ & 4 & $90.2-102$ & 96.0 & Yes \\
\hline Manganese, unfiltered recoverable, $\mu \mathrm{g} / \mathrm{L}$ & 4 & 78.8-109 & 93.9 & Yes \\
\hline Manganese, filtered, $\mu \mathrm{g} / \mathrm{L}$ & 4 & $86.2-112$ & 99.3 & Yes \\
\hline Zinc, unfiltered recoverable, $\mu \mathrm{g} / \mathrm{L}$ & 4 & $87.5-98.5$ & 93.0 & Yes \\
\hline Zinc, filtered, $\mu \mathrm{g} / \mathrm{L}$ & 4 & 93.6-103 & 98.4 & Yes \\
\hline
\end{tabular}


54 Water-Quality, Bed-Sediment, and Biological Data (October 2002 through September 2003) and Statistical Summaries of Data for Streams in the Upper Clark Fork Basin, Montana

Table 13. Chemical analyses of field blanks for water samples

[The terms "filtered" and "unfiltered recoverable" replace the former terms "dissolved" and "total recoverable," respectively. Abbreviations: ${ }^{\circ} \mathrm{C}$, degrees Celsius; E, estimated; $\mu \mathrm{g} / \mathrm{L}$, micrograms per liter; $\mu \mathrm{S} / \mathrm{cm}$, microsiemens per centimeter at $25^{\circ} \mathrm{C}$; mg/L, milligrams per liter. Symbols:

$<$, less than analytical detection limit]

\begin{tabular}{|c|c|c|c|c|c|c|c|c|c|}
\hline Date & Time & $\begin{array}{c}\mathrm{pH}, \\
\text { onsite } \\
\text { (standard } \\
\text { units) }\end{array}$ & $\begin{array}{c}\text { Specific } \\
\text { conductance, } \\
\text { onsite } \\
(\mu \mathrm{S} / \mathrm{cm})\end{array}$ & $\begin{array}{c}\text { Calcium, } \\
\text { filtered } \\
\text { (mg/L) }\end{array}$ & $\begin{array}{c}\text { Magnesium, } \\
\text { filtered } \\
(\mathrm{mg} / \mathrm{L})\end{array}$ & $\begin{array}{c}\text { Arsenic, } \\
\text { filtered } \\
(\mu \mathrm{g} / \mathrm{L})\end{array}$ & $\begin{array}{c}\text { Arsenic, } \\
\text { unfiltered } \\
\text { recoverable } \\
\langle\mu \mathrm{g} / \mathrm{L}\rangle\end{array}$ & $\begin{array}{c}\text { Cadmium, } \\
\text { filtered } \\
(\mu \mathrm{g} / \mathrm{L})\end{array}$ & $\begin{array}{c}\text { Cadmium, } \\
\text { unfiltered } \\
\text { recoverable } \\
(\mu \mathrm{g} / \mathrm{L})\end{array}$ \\
\hline $28 \ldots$ & 1200 & 5.6 & 2 & E0.01 & $<0.008$ & $<0.3$ & $<2$ & 0.34 & $<0.04$ \\
\hline \multicolumn{10}{|l|}{ APR } \\
\hline $03 \ldots$ & 1500 & 5.5 & 3 & .02 & $<.008$ & $<.3$ & $<2$ & $<.04$ & $<.04$ \\
\hline \multicolumn{10}{|l|}{ JUN } \\
\hline $01 \ldots$ & 1800 & 5.7 & 2 & .01 & $<.008$ & $<.3$ & $<2$ & $<.04$ & $<.04$ \\
\hline $16 \ldots$ & 1100 & 5.6 & 3 & E.01 & $<.008$ & $<.3$ & $<2$ & $<.04$ & $<.04$ \\
\hline \multicolumn{10}{|l|}{ JUL } \\
\hline $28 \ldots$ & 1800 & 5.8 & 1 & E.01 & E.003 & $<.3$ & $<2$ & $<.04$ & $<.04$ \\
\hline \multicolumn{10}{|l|}{ AUG } \\
\hline
\end{tabular}

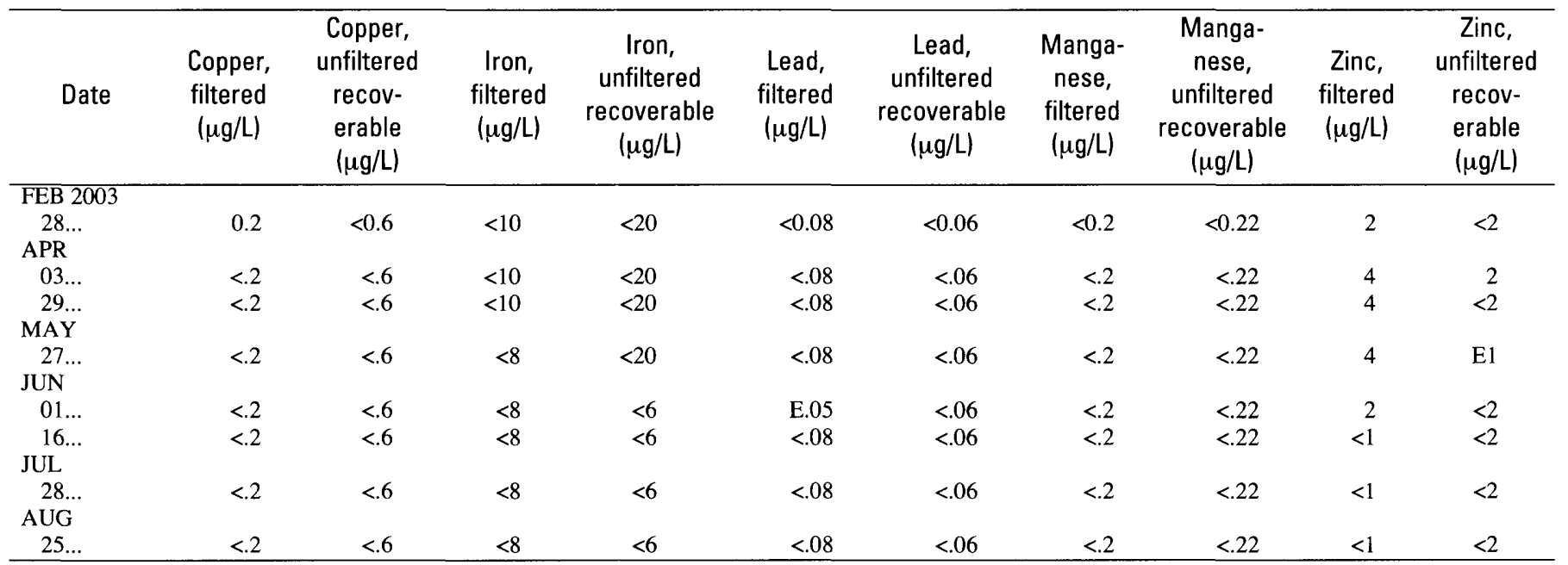


Table 14. Trace-element analyses of fine-grained bed sediment, upper Clark Fork basin, Montana, September 2003

[Fine-grained sediment is material less than 0.064 millimeter in diameter. Reported concentrations are the mean of all analyses for replicate aliquots from each composite sample. Abbreviation: $\mu \mathrm{g} / \mathrm{g}$, micrograms per gram of dry sample weight]

\begin{tabular}{|c|c|c|c|c|c|c|c|c|c|c|c|}
\hline \multirow{2}{*}{$\begin{array}{l}\text { Station number } \\
\text { (fig. 1) }\end{array}$} & \multirow{2}{*}{ Station name } & \multirow{2}{*}{$\begin{array}{l}\text { Number } \\
\text { of com- } \\
\text { posite } \\
\text { samples }\end{array}$} & \multicolumn{9}{|c|}{ Concentration, in $\mu \mathrm{g} / \mathrm{g}$} \\
\hline & & & Arsenic & $\begin{array}{l}\text { Cad- } \\
\text { mium }\end{array}$ & $\begin{array}{l}\text { Cop- } \\
\text { per }\end{array}$ & Iron & Lead & $\begin{array}{l}\text { Manga- } \\
\text { nese }\end{array}$ & Nickel & Silver & Zinc \\
\hline 12323600 & Silver Bow Creek at Opportunity & 3 & 186 & 41.9 & 3,400 & 32,200 & 581 & 5,220 & 15.3 & 11.3 & 7,630 \\
\hline 12323750 & Silver Bow Creek at Warm Springs & 3 & 103 & 7.4 & 196 & 19,100 & 73 & 14,400 & 13.0 & 4.4 & 726 \\
\hline 12323800 & Clark Fork near Galen & 3 & 111 & 10.0 & 1,030 & 26,200 & 137 & 12,600 & 18.3 & 5.5 & 1,130 \\
\hline 461415112450801 & Clark Fork below Lost Creek, near Galen & 3 & 204 & 9.4 & 1,600 & 30,300 & 218 & 9,670 & 16.3 & 7.6 & 1,560 \\
\hline 461559112443301 & Clark Fork near Racetrack & 3 & 101 & 8.7 & 1,310 & 28,100 & 178 & 6,310 & 15.6 & 5.8 & 1,380 \\
\hline 461903112440701 & $\begin{array}{l}\text { Clark Fork at Dempsey Creek } \\
\text { diversion, near Racetrack }\end{array}$ & 3 & 80 & 9.0 & 1,090 & 25,200 & 155 & 8,370 & 12.9 & 5.7 & 1,230 \\
\hline 12324200 & Clark Fork at Deer Lodge & 3 & 77 & 9.6 & 1,080 & 24,300 & 148 & 3,980 & 13.6 & 4.7 & 1,260 \\
\hline 12324680 & Clark Fork at Goldcreek & 3 & 32 & 5.8 & 438 & 19,500 & 68 & 2,180 & 12.2 & 2.4 & 669 \\
\hline 12331500 & Flint Creek near Drummond & 3 & 113 & 6.2 & 47 & 23,200 & 175 & 5,720 & 11.4 & 6.3 & 626 \\
\hline 12331800 & Clark Fork near Drummond & 3 & 33 & 5.3 & 321 & 17,400 & 68 & 3,090 & 10.8 & 2.7 & 761 \\
\hline 12334510 & Rock Creek near Clinton & 3 & 5 & 2.8 & 6 & 16,800 & 11 & 490 & 11.3 & .8 & 23 \\
\hline 12334550 & $\begin{array}{l}\text { Clark Fork at Turah Bridge, } \\
\text { near Bonner }\end{array}$ & 3 & 19 & 3.9 & 211 & 12,600 & 48 & 1,410 & 8.7 & 1.7 & 663 \\
\hline 12340000 & Blackfoot River near Bonner & 3 & 2 & 1.8 & 11 & 14,800 & 11 & 605 & 9.8 & .7 & 39 \\
\hline 12340500 & Clark Fork above Missoula & 3 & 23 & 5.8 & 326 & 19,200 & 54 & 1,420 & 12.7 & 2.1 & 716 \\
\hline 12353000 & Clark Fork below Missoula ${ }^{1}$ & 3 & 6 & 3.0 & 69 & 13,100 & 24 & 1,420 & 8.4 & 1.0 & 239 \\
\hline
\end{tabular}

${ }^{1}$ Samples collected about 30 miles downstream from streamflow-gaging station to conform to previous sampling location. 
[Bulk bed sediment collected in this study generally is material smaller than about 10 millimeters in diameter. Reported concentrations are the mean of all analyses for replicate aliquots of each composite sample. Abbreviation: $\mu \mathrm{g} / \mathrm{g}$, micrograms per gram of dry sample weight. Symbol: <, less than]

\begin{tabular}{|c|c|c|c|c|c|c|c|c|c|c|c|}
\hline \multirow{2}{*}{$\begin{array}{l}\text { Station number } \\
\text { (fig. 1) }\end{array}$} & \multirow{2}{*}{ Station name } & \multirow{2}{*}{$\begin{array}{l}\text { Number } \\
\text { of com- } \\
\text { posite } \\
\text { samples }\end{array}$} & \multicolumn{9}{|c|}{ Concentration, in $\mu \mathrm{g} / \mathrm{g}$} \\
\hline & & & Arsenic & $\begin{array}{l}\text { Cad- } \\
\text { mium }\end{array}$ & Copper & Iron & Lead & $\begin{array}{c}\text { Manga- } \\
\text { nese }\end{array}$ & Nickel & Silver & Zinc \\
\hline 12323600 & Silver Bow Creek at Opportunity & 1 & 148 & 21.7 & 1,850 & 25,700 & 392 & 2,900 & 9.8 & 5.9 & 3,480 \\
\hline 12323750 & Silver Bow Creek at Warm Springs & 1 & 36 & 3.4 & 60 & 13,200 & 30 & 1,990 & 10.3 & .9 & 220 \\
\hline 12323800 & Clark Fork near Galen & 1 & 97 & 4.7 & 540 & 15,100 & 94 & 3,130 & 7.2 & 1.7 & 510 \\
\hline 461415112450801 & Clark Fork below Lost Creek, near Galen & 1 & 82 & 5.2 & 420 & 18,300 & 71 & 3,720 & 8.4 & 2.0 & 560 \\
\hline 461559112443301 & Clark Fork near Racetrack & 1 & 90 & 10.5 & 600 & 15,700 & 85 & 4,170 & 9.9 & 2.5 & 1,000 \\
\hline 461903112440701 & $\begin{array}{l}\text { Clark Fork at Dempsey Creek diversion, } \\
\text { near Racetrack }\end{array}$ & 1 & 82 & 5.6 & 640 & 16,700 & 94 & 3,070 & 7.9 & 2.3 & 610 \\
\hline 12324200 & Clark Fork at Deer Lodge & 1 & 84 & 8.3 & 820 & 22,000 & 122 & 3,560 & 11.8 & 3.5 & 950 \\
\hline 12324680 & Clark Fork at Goldcreek & 1 & 15 & 3.2 & 170 & 11,900 & 56 & 930 & 8.3 & 1.0 & 310 \\
\hline 12331500 & Flint Creek near Drummond & 1 & 80 & 3.6 & 20 & 14,700 & 111 & 2,690 & 7.1 & 4.3 & 360 \\
\hline 12331800 & Clark Fork near Drummond & 1 & 31 & 5.1 & 310 & 16,000 & 65 & 3,280 & 10.3 & 2.6 & 680 \\
\hline 12334510 & Rock Creek near Clinton & 1 & 1 & .5 & $<.6$ & 5,300 & 2 & 60 & 4.0 & .4 & .3 \\
\hline 12334550 & Clark Fork at Turah Bridge, near Bonner & 1 & 18 & 5.5 & 250 & 15,400 & 46 & 2,620 & 10.9 & 1.8 & 590 \\
\hline 12340000 & Blackfoot River near Bonner & 1 & $<.5$ & 2.5 & 10 & 13,000 & 5 & 330 & 8.7 & .8 & 20 \\
\hline 12340500 & Clark Fork above Missoula & 1 & 4 & 2.4 & 80 & 11,100 & 20 & 570 & 8.1 & .9 & 290 \\
\hline 12353000 & Clark Fork below Missoula ${ }^{1}$ & 1 & 1.1 & 1.2 & 10 & 8,400 & 5 & 220 & 4.6 & .3 & 40 \\
\hline
\end{tabular}

${ }^{1}$ Samples collected about 30 miles downstream from streamflow-gaging station to conform to previous sampling location. 
Table 16. Recovery efficiency for trace-element analyses of standard reference materials for bed sediment

[Abbreviations: $\mu \mathrm{g} / \mathrm{g}$, micrograms per gram of dry sample weight; SRM, standard reference material. Symbol: --, recovery could not be determined because all analyses were less than the analytical detection limit]

\begin{tabular}{|c|c|c|c|c|c|}
\hline Constituent & $\begin{array}{c}\text { Number of } \\
\text { measure- } \\
\text { ments }\end{array}$ & $\begin{array}{c}\text { Dilution } \\
\text { ratio }\end{array}$ & $\begin{array}{c}\text { Certified } \\
\text { concentration } \\
(\mu \mathrm{g} / \mathrm{g})\end{array}$ & $\begin{array}{l}\text { Mean SRM } \\
\text { recovery } \\
\text { (percent) }\end{array}$ & $\begin{array}{l}\text { 95-percent confidence } \\
\text { interval for SRM } \\
\text { recovery } \\
\text { (percent) }\end{array}$ \\
\hline \multicolumn{6}{|c|}{ SRM sample 2709 } \\
\hline Arsenic & 7 & $1: 5$ & 17.7 & 68.1 & $67.8-68.4$ \\
\hline Cadmium & 7 & $1: 5$ & .4 & -- & -- \\
\hline Copper & 7 & $1: 5$ & 35 & 50.4 & $48.8-52.3$ \\
\hline Iron & 7 & $1: 5$ & 35,000 & 88.4 & $87.5-89.4$ \\
\hline Lead & 7 & $1: 5$ & 19 & 83.1 & $79.2-87.0$ \\
\hline Manganese & 7 & $1: 5$ & 538 & 94.4 & $93.4-95.4$ \\
\hline Nickel & 7 & $1: 5$ & 88 & 90.6 & $89.6-91.6$ \\
\hline Silver & 7 & $1: 5$ & .4 & - & -- \\
\hline Zinc & 7 & $1: 5$ & 106 & 76.2 & $72.2-80.0$ \\
\hline \multicolumn{6}{|c|}{ SRM sample 2711} \\
\hline Arsenic & 7 & $1: 10$ & 105 & 84.4 & $80.7-88.1$ \\
\hline Cadmium & 7 & $1: 10$ & 41.7 & 115.6 & $110-121$ \\
\hline Copper & 7 & $1: 10$ & 114 & 66.2 & $64.0-68.3$ \\
\hline Iron & 7 & $1: 10$ & 28,900 & 86.5 & $83.4-89.6$ \\
\hline Lead & 7 & $1: 10$ & 1,160 & 108.4 & $104-112$ \\
\hline Manganese & 7 & $1: 10$ & 638 & 88.2 & 85.1-91.4 \\
\hline Nickel & 7 & $1: 10$ & 20.6 & 90.7 & $86.8-94.6$ \\
\hline Silver & 7 & $1: 10$ & 4.6 & 78.5 & $75.9-79.6$ \\
\hline Zinc & 7 & $1: 10$ & 350 & 88.0 & $84.3-91.7$ \\
\hline
\end{tabular}




\section{Table 17. Trace-element analyses of procedural blanks for bed sediment}

[Abbreviation: $\mu \mathrm{g} / \mathrm{mL}$, micrograms per milliliter. Dilution ratio is the proportion of initial volume of concentrated nitric acid used as a digesting reagent to final volume of solution after addition of $0.6 \mathrm{~N}$ (normal) hydrochloric acid used for reconstituting dried residue. Symbol: <, less than]

\begin{tabular}{|c|c|c|c|c|c|c|c|c|c|c|c|}
\hline \multirow[b]{2}{*}{ Station number } & \multirow[b]{2}{*}{ Station name } & \multirow{2}{*}{$\begin{array}{l}\text { Dilution } \\
\text { ratio }\end{array}$} & \multicolumn{9}{|c|}{ Trace-element concentration, in $\mu \mathrm{g} / \mathrm{mL}$} \\
\hline & & & Arsenic & $\begin{array}{l}\text { Cad- } \\
\text { mium }\end{array}$ & $\begin{array}{l}\text { Cop- } \\
\text { per }\end{array}$ & Iron & Lead & $\begin{array}{c}\text { Manga- } \\
\text { nese }\end{array}$ & Nickel & Silver & Zinc \\
\hline 12323600 & Silver Bow Creek at Opportunity & $1: 1$ & $<0.003$ & 0.0001 & $<0.003$ & $<0.004$ & $<0.004$ & $<0.001$ & $<0.001$ & $<0.001$ & $<0.002$ \\
\hline 12323750 & Silver Bow Creek at Warm Springs & 1:1 & $<.003$ & $<.0001$ & $<.003$ & $<.004$ & $<.004$ & $<.001$ & $<.001$ & $<.001$ & $<.002$ \\
\hline 12323800 & Clark Fork near Galen & $1: 1$ & $<.003$ & $<.0001$ & $<.003$ & $<.004$ & $<.004$ & $<.001$ & $<.001$ & $<.001$ & $<.002$ \\
\hline 461415112450801 & Clark Fork below Lost Creek, near Galen & $1: 1$ & $<.003$ & $<.0001$ & $<.003$ & $<.004$ & $<.004$ & $<.001$ & $<.001$ & $<.001$ & $<.002$ \\
\hline 461559112443301 & Clark Fork near Racetrack & $1: 1$ & $<.003$ & $<.0001$ & $<.003$ & $<.004$ & $<.004$ & $<.001$ & $<.001$ & $<.001$ & $<.002$ \\
\hline 461903112440701 & $\begin{array}{l}\text { Clark Fork at Dempsey Creek diversion, near } \\
\text { Racetrack }\end{array}$ & $1: 1$ & $<.003$ & $<.0001$ & $<.003$ & $<.004$ & $<.004$ & $<.001$ & $<.001$ & $<.001$ & $<.002$ \\
\hline 12324200 & Clark Fork at Deer Lodge & $1: 1$ & $<.003$ & $<.0001$ & $<.003$ & $<.004$ & $<.004$ & $<.001$ & $<.001$ & $<.001$ & $<.002$ \\
\hline 12324680 & Clark Fork at Goldcreek & $1: 1$ & $<.003$ & $<.0001$ & $<.003$ & $<.004$ & $<.004$ & $<.001$ & $<.001$ & $<.001$ & $<.002$ \\
\hline 12331500 & Flint Creek near Drummond & $1: 1$ & $<.003$ & $<.0001$ & $<.003$ & $<.004$ & $<.004$ & $<.001$ & $<.001$ & $<.001$ & $<.002$ \\
\hline 12331800 & Clark Fork near Drummond & $1: 1$ & $<.003$ & $<.0001$ & $<.003$ & $<.004$ & $<.004$ & $<.001$ & $<.001$ & $<.001$ & $<.002$ \\
\hline 12334510 & Rock Creek near Clinton & $1: 1$ & $<.003$ & $<.0001$ & $<.003$ & $<.004$ & $<.004$ & $<.001$ & $<.001$ & $<.001$ & $<.002$ \\
\hline 12334550 & Clark Fork at Turah Bridge, near Bonner & $1: 1$ & $<.003$ & $<.0001$ & $<.003$ & $<.004$ & $<.004$ & $<.001$ & $<.001$ & $<.001$ & $<.002$ \\
\hline 12340000 & Blackfoot River near Bonner & $1: 1$ & $<.003$ & $<.0001$ & $<.003$ & $<.004$ & $<.004$ & $<.001$ & $<.001$ & $<.001$ & $<.002$ \\
\hline 12340500 & Clark Fork above Missoula & $1: 1$ & $<.003$ & $<.0001$ & $<.003$ & $<.004$ & $<.004$ & $<.001$ & $<.001$ & $<.001$ & $<.002$ \\
\hline 12353000 & Clark Fork below Missoula & $1: 1$ & $<.003$ & $<.0001$ & $<.003$ & $<.004$ & $<.004$ & $<.001$ & $<.001$ & $<.001$ & $<.002$ \\
\hline
\end{tabular}


Table 18. Trace-element analyses of biota, upper Clark Fork basin, Montana, September 2003

[Analyses are of whole-body tissue of aquatic insects. Composite samples made by combining similar-sized insects of the same species into a sample of sufficient mass for analysis. Concentrations for biota samples composed of two or more composite samples are the means of all analyses. Abbreviations: $\mu \mathrm{g} / \mathrm{g}$, micrograms per gram of dry sample weight; spp., species. Symbol: <, less than analytical detection limit]

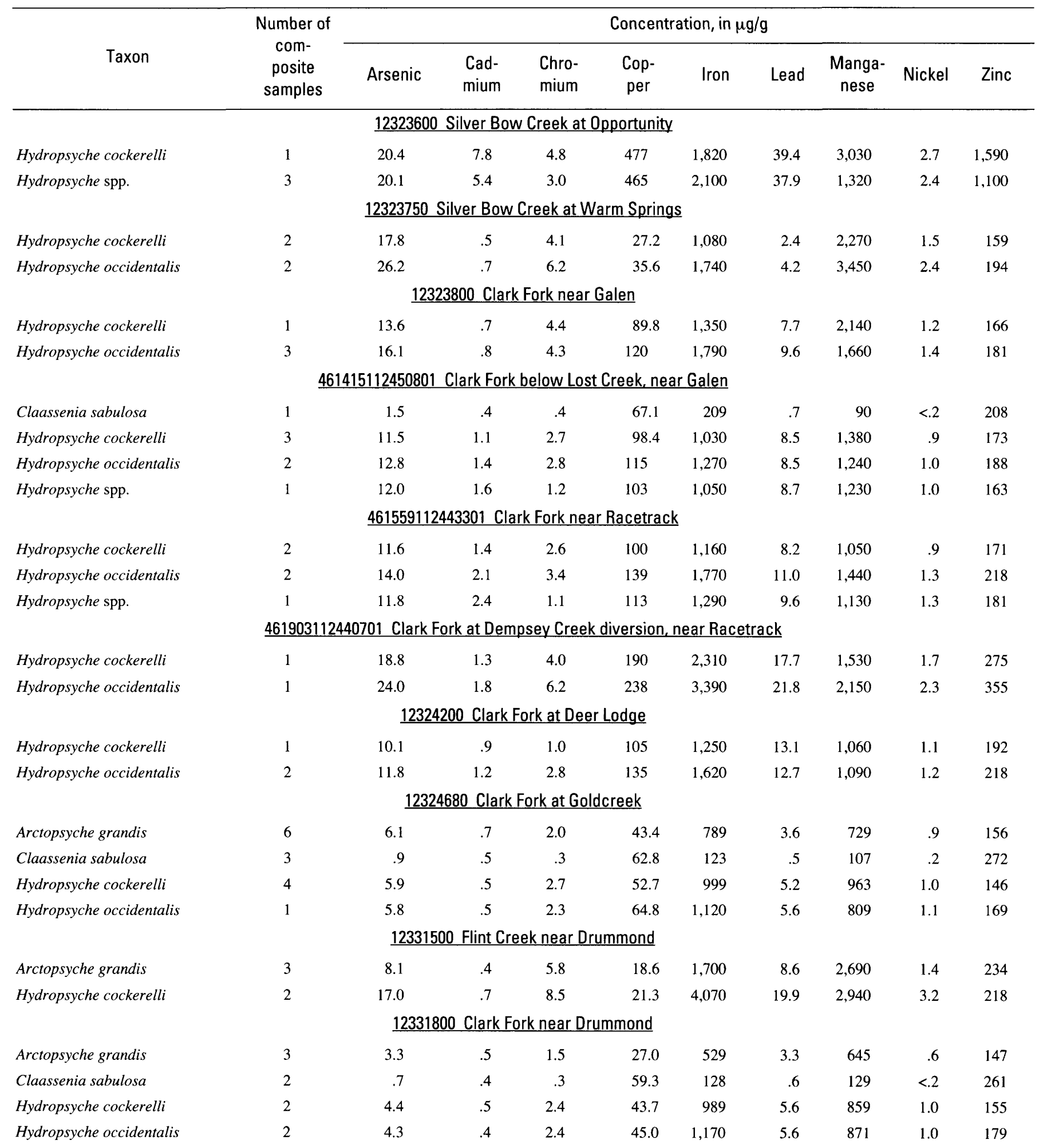


60 Water-Quality, Bed-Sediment, and Biological Data (October 2002 through September 2003) and Statistical Summaries of Data for Streams in the Upper Clark Fork Basin, Montana

Table 18. Trace-element analyses of biota, upper Clark Fork basin, Montana, September 2003-Continued

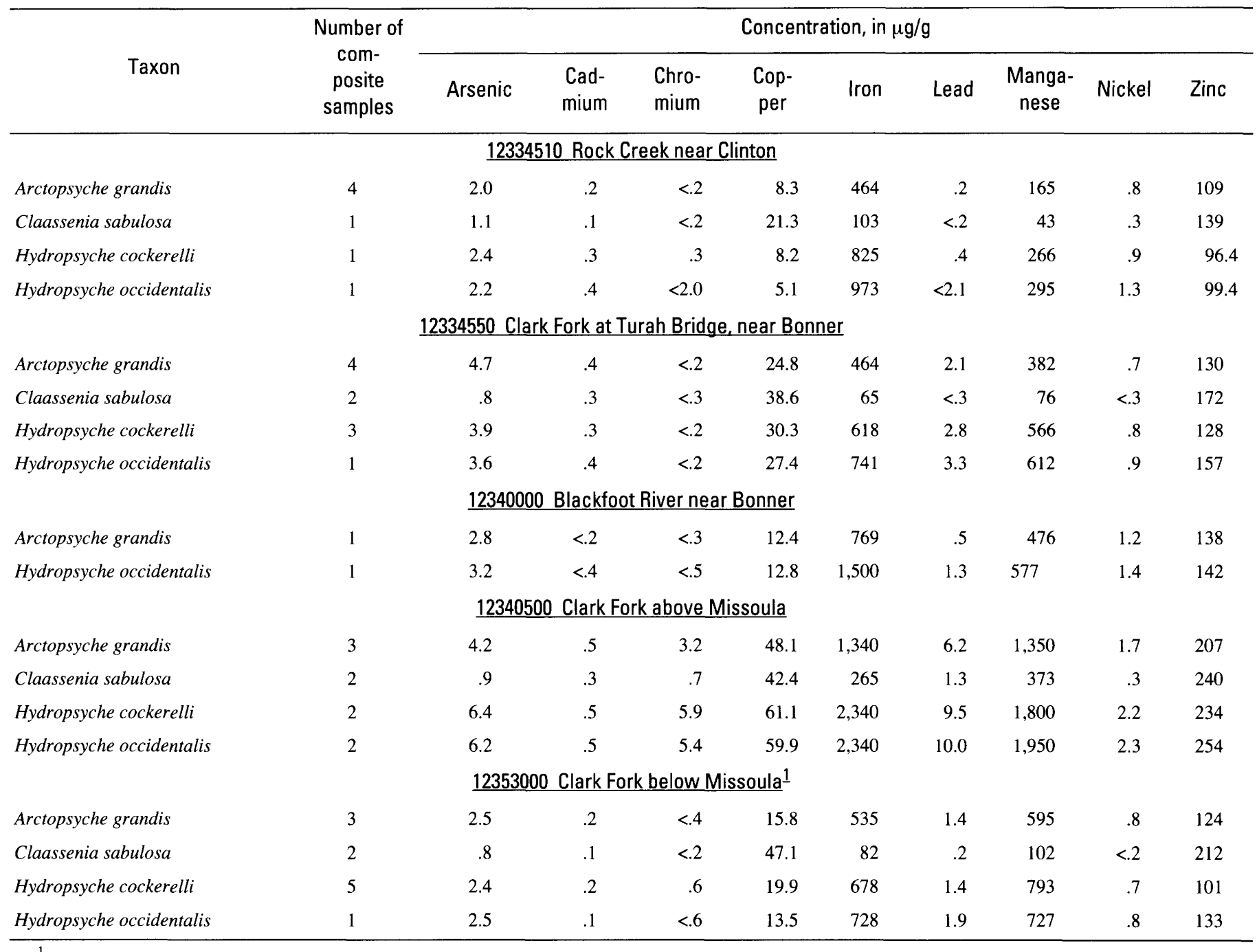

${ }^{1}$ Samples collected about 30 miles downstream from streamflow-gaging station to conform to previous sampling location. 
Table 19. Recovery efficiency for trace-element analyses of standard reference material for biota [Abbreviations: $\mu \mathrm{g} / \mathrm{g}$, micrograms per gram of dry sample weight; $\mu \mathrm{g} / \mathrm{mL}$, micrograms per milliliter; SRM, standard reference material]

\begin{tabular}{|c|c|c|c|c|}
\hline Constituent & $\begin{array}{c}\text { Number of } \\
\text { measurements }\end{array}$ & $\begin{array}{c}\text { Certified } \\
\text { concentration } \\
(\mu \mathrm{g} / \mathrm{g})\end{array}$ & $\begin{array}{c}\text { Mean SRM } \\
\text { recovery } \\
\text { (percent) }\end{array}$ & $\begin{array}{l}\text { 95-percent confidence } \\
\text { interval for SRM recovery } \\
\text { (percent) }\end{array}$ \\
\hline \multicolumn{5}{|c|}{ SRM sample TORT-2 } \\
\hline Arsenic & 12 & 21.6 & 101 & $98.8-104$ \\
\hline Cadmium & 12 & 26.7 & 88.8 & $85.2-92.4$ \\
\hline Chromium & ${ }^{1} 12$ & .77 & 74.3 & $19.1-129$ \\
\hline Copper & 12 & 106 & 92.5 & $90.4-94.7$ \\
\hline Iron & 12 & 105 & 94.9 & $90.2-100$ \\
\hline Lead & ${ }^{2} 12$ & .35 & 48.4 & $17.2-79.7$ \\
\hline Manganese & 12 & 13.6 & 88.3 & $83.7-92.8$ \\
\hline Nickel & 12 & 2.5 & 80.4 & $73.5-87.4$ \\
\hline Zinc & 12 & 180 & 93.3 & $89.4-97.2$ \\
\hline
\end{tabular}

${ }^{1}$ Chromium concentrations in six samples were less than the analytical detection limit $(0.03 \mu \mathrm{g} / \mathrm{mL})$.

${ }^{2} \mathrm{Lead}$ concentrations in seven samples were less than the analytical detection limit $(0.01 \mu \mathrm{g} / \mathrm{mL})$. 
62 Water-Quality, Bed-Sediment, and Biological Data (October 2002 through September 2003) and Statistical Summaries of Data for Streams in the Upper Clark Fork Basin, Montana

Table 20. Trace-element analyses of procedural blanks for biota

[Procedural blanks were not diluted prior to analysis. Abbreviation: $\mu \mathrm{g} / \mathrm{mL}$, micrograms per milliliter. Symbol: <, less than]

\begin{tabular}{|c|c|c|c|c|c|c|c|c|c|c|c|}
\hline \multirow{2}{*}{$\begin{array}{l}\text { Station } \\
\text { number }\end{array}$} & \multirow{2}{*}{$\begin{array}{l}\text { Station } \\
\text { name }\end{array}$} & \multirow{2}{*}{$\begin{array}{l}\text { Dilution } \\
\text { ratio }\end{array}$} & \multicolumn{9}{|c|}{ Trace-element concentration, in $\mu \mathrm{g} / \mathrm{mL}$} \\
\hline & & & Arsenic & $\begin{array}{l}\text { Cad- } \\
\text { mium }\end{array}$ & $\begin{array}{l}\text { Chro- } \\
\text { mium }\end{array}$ & Copper & Iron & Lead & $\begin{array}{c}\text { Manga- } \\
\text { nese }\end{array}$ & Nickel & Zinc \\
\hline 12323600 & Silver Bow Creek at Opportunity & $1: 1$ & $<0.01$ & $<0.002$ & $<0.03$ & $<0.01$ & $<0.06$ & $<0.01$ & $<0.01$ & $<0.01$ & $<0.01$ \\
\hline 12323750 & Silver Bow Creek at Warm Springs & $1: 1$ & $<.01$ & $<.002$ & $<.03$ & $<.01$ & $<.06$ & $<.01$ & $<.01$ & $<.01$ & $<.01$ \\
\hline 12323800 & Clark Fork near Galen & $1: 1$ & $<.01$ & $<.002$ & $<.03$ & $<.01$ & $<.06$ & $<.01$ & $<.01$ & $<.01$ & $<.01$ \\
\hline 461415112450801 & Clark Fork below Lost Creek, near Galen & $1: 1$ & $<.01$ & $<.002$ & $<.03$ & $<.01$ & $<.06$ & $<.01$ & $<.01$ & $<.01$ & $<.01$ \\
\hline 461559112443301 & Clark Fork near Racetrack & $1: 1$ & $<.01$ & $<.002$ & $<.03$ & $<.01$ & $<.06$ & $<.01$ & $<.01$ & $<.01$ & $<.01$ \\
\hline 461903112440701 & $\begin{array}{l}\text { Clark Fork at Dempsey Creek diversion, } \\
\text { near Racetrack }\end{array}$ & $1: 1$ & $<.01$ & $<.002$ & $<.03$ & $<.01$ & $<.06$ & $<.01$ & $<.01$ & $<.01$ & $<.01$ \\
\hline 12324200 & Clark Fork at Deer Lodge & $1: 1$ & $<.01$ & $<.002$ & $<.03$ & $<.01$ & $<.06$ & $<.01$ & $<.01$ & $<.01$ & $<.01$ \\
\hline 12324680 & Clark Fork at Goldcreek & $1: 1$ & $<.01$ & $<.002$ & $<.03$ & $<.01$ & $<.06$ & $<.01$ & $<.01$ & $<.01$ & $<.01$ \\
\hline 12331500 & Flint Creek near Drummond & $1: 1$ & $<.01$ & $<.002$ & $<.03$ & $<.01$ & $<.06$ & $<.01$ & $<.01$ & $<.01$ & $<.01$ \\
\hline 12331800 & Clark Fork near Drummond & $1: 1$ & $<.01$ & $<.002$ & $<.03$ & $<.01$ & $<.06$ & $<.01$ & $<.01$ & $<.01$ & $<.01$ \\
\hline 12334510 & Rock Creek near Clinton & $1: 1$ & $<.01$ & $<.002$ & $<.03$ & $<.01$ & $<.06$ & $<.01$ & $<.01$ & $<.01$ & $<.01$ \\
\hline 12334550 & Clark Fork at Turah Bridge, near Bonner & $1: 1$ & $<.01$ & $<.002$ & $<.03$ & $<.01$ & $<.06$ & $<.01$ & $<.01$ & $<.01$ & $<.01$ \\
\hline 12340000 & Blackfoot River near Bonner & $1: 1$ & $<.01$ & $<.002$ & $<.03$ & $<.01$ & $<.06$ & $<.01$ & $<.01$ & $<.01$ & $<.01$ \\
\hline 12340500 & Clark Fork above Missoula & $1: 1$ & $<.01$ & $<.002$ & $<.03$ & $<.01$ & $<.06$ & $<.01$ & $<.01$ & $<.01$ & $<.01$ \\
\hline 12353000 & Clark Fork below Missoula & $1: 1$ & $<.01$ & $<.002$ & $<.03$ & $<.01$ & $<.06$ & $<.01$ & $<.01$ & $<.01$ & $<.01$ \\
\hline
\end{tabular}


Table 21. Statistical summary of water-quality data for the upper Clark Fork basin, Montana, March 1985 through September 2003

[The terms "filtered" and "unfiltered recoverable" replace the former terms "dissolved" and "total recoverable," respectively. Abbreviations: $\mathrm{ft}^{3} / \mathrm{s}$, cubic feet per second; ${ }^{\circ} \mathrm{C}$, degrees Celsius; E, estimated; $\mu \mathrm{g} / \mathrm{L}$, micrograms per liter; $\mu \mathrm{S} / \mathrm{cm}$, microsiemens per centimeter at $25^{\circ} \mathrm{C} ; \mathrm{mg} / \mathrm{L}$, milligrams per liter; $\mathrm{mm}$, millimeter; ton/d, tons per day. Symbols: <, less than analytical detection limit ${ }^{1}$; --, indicates insufficient data greater than the analytical detection limit to compute statistiC]

\begin{tabular}{|c|c|c|c|c|c|}
\hline Property or constituent and reporting unit & $\begin{array}{c}\text { Number of } \\
\text { samples }\end{array}$ & Maximum & Minimum & Mean & Median \\
\hline \multicolumn{6}{|c|}{ 12323230--BLACKTAIL CREEK AT HARRISON AVENUE, AT BUTTE, MONT. } \\
\hline \multicolumn{6}{|c|}{ Period of record for water-quality data: March 1993-August 1995, December 1996-August 2003} \\
\hline Streamflow, instantaneous $\left(\mathrm{ft}^{3} / \mathrm{s}\right)$ & 83 & 156 & 1.9 & 14 & 7.9 \\
\hline Specific conductance, onsite $(\mu \mathrm{S} / \mathrm{cm})$ & 83 & 412 & 116 & 267 & 271 \\
\hline Temperature, water $\left({ }^{\circ} \mathrm{C}\right)$ & 83 & 17.5 & 1.5 & 8.2 & 8.0 \\
\hline $\mathrm{pH}$, onsite (standard units) & 83 & 8.4 & 7.3 & 7.8 & 7.8 \\
\hline Hardness, filtered $\left(\mathrm{mg} / \mathrm{L}\right.$ as $\left.\mathrm{CaCO}_{3}\right)$ & 83 & 150 & 38 & 105 & 110 \\
\hline Calcium, filtered (mg/L) & 83 & 41.8 & 10.6 & 30 & 31 \\
\hline Magnesium, filtered (mg/L) & 83 & 11.0 & 2.71 & 7.3 & 7.4 \\
\hline Arsenic, unfiltered recoverable $(\mu \mathrm{g} / \mathrm{L})$ & 83 & 18 & $<2$ & 25 & 4 \\
\hline Arsenic, filtered $(\mu \mathrm{g} / \mathrm{L})$ & 83 & 13 & 1 & 4 & 3 \\
\hline Cadmium, unfiltered recoverable $(\mu \mathrm{g} / \mathrm{L})$ & 83 & .11 & $<.04$ & ${ }^{2}<1$ & $<1$ \\
\hline Cadmium, filtered $(\mu \mathrm{g} / \mathrm{L})$ & 83 & .5 & $<.1$ & ${ }^{2}<.1$ & $<.1$ \\
\hline Copper, unfiltered recoverable $(\mu \mathrm{g} / \mathrm{L})$ & 83 & 52 & 1.5 & 7 & 6 \\
\hline Copper, filtered $(\mu \mathrm{g} / \mathrm{L})$ & 83 & 10 & E. 8 & ${ }^{2} 4$ & 3 \\
\hline Iron, unfiltered recoverable $(\mu \mathrm{g} / \mathrm{L})$ & 83 & 4,220 & 140 & 700 & 550 \\
\hline Iron, filtered $(\mu \mathrm{g} / \mathrm{L})$ & 83 & 478 & 15 & 163 & 157 \\
\hline Lead, unfiltered recoverable $(\mu \mathrm{g} / \mathrm{L})$ & 83 & 47 & $<1$ & ${ }^{2} 2$ & 1 \\
\hline Lead, filtered $(\mu \mathrm{g} / \mathrm{L})$ & 83 & 1 & $<.08$ & ${ }^{2} .2$ & $<.5$ \\
\hline Manganese, unfiltered recoverable $(\mu \mathrm{g} / \mathrm{L})$ & 83 & 240 & 24 & 62 & 53 \\
\hline Manganese, filtered $(\mu \mathrm{g} / \mathrm{L})$ & 83 & 144 & 17 & 44 & 39 \\
\hline Zinc, unfiltered recoverable $(\mu \mathrm{g} / \mathrm{L})$ & 83 & 130 & $<10$ & ${ }^{2} 11$ & 3 \\
\hline Zinc, filtered $(\mu \mathrm{g} / \mathrm{L})$ & 83 & 11 & $<1$ & 24 & 3 \\
\hline Sediment, suspended concentration $(\mathrm{mg} / \mathrm{L})$ & 83 & 139 & 2 & 15 & 7 \\
\hline Sediment, suspended discharge (ton/d) & 83 & 59 & .02 & 1.4 & .14 \\
\hline Sediment, suspended (percent finer than $0.062 \mathrm{~mm}$ ) & 83 & 97 & 50 & 83 & 84 \\
\hline
\end{tabular}


64 Water-Quality, Bed-Sediment, and Biological Data (October 2002 through September 2003) and Statistical Summaries of Data for Streams in the Upper Clark Fork Basin, Montana

Table 21. Statistical summary of water-quality data for the upper Clark Fork basin, Montana, March 1985 through September 2003-Continued

\begin{tabular}{|c|c|c|c|c|c|}
\hline Property or constituent and reporting unit & $\begin{array}{l}\text { Number of } \\
\text { samples }\end{array}$ & Maximum & Minimum & Mean & Median \\
\hline \multicolumn{6}{|c|}{ 12323250--SILVER BOW CREEK BELOW BLACKTAIL CREEK, AT BUTTE, MONT. } \\
\hline \multicolumn{6}{|c|}{ Period of record for water-quality data: March 1993-August 1995, December 1996-September 2003} \\
\hline Streamflow, instantaneous $\left(\mathrm{ft}^{3} / \mathrm{s}\right)$ & 83 & 134 & 13 & 30 & 24 \\
\hline Specific conductance, onsite $(\mu \mathrm{S} / \mathrm{cm})$ & 83 & 691 & 226 & 469 & 478 \\
\hline Temperature, water $\left({ }^{\circ} \mathrm{C}\right)$ & 83 & 20.0 & 1.0 & 10.3 & 9.5 \\
\hline pH, onsite (standard units) & 83 & 8.1 & 7.2 & 7.6 & 7.6 \\
\hline Hardness, filtered (mg/L as $\mathrm{CaCO}_{3}$ ) & 83 & 180 & 66 & 148 & 150 \\
\hline Calcium, filtered (mg/L) & 83 & 51.6 & 19.0 & 42 & 43 \\
\hline Magnesium, filtered (mg/L) & 83 & 13.0 & 4.51 & 10 & 11 \\
\hline Arsenic, unfiltered recoverable $(\mu \mathrm{g} / \mathrm{L})$ & 83 & 45 & 4 & 13 & 11 \\
\hline Arsenic, filtered $(\mu \mathrm{g} / \mathrm{L})$ & 83 & 13 & 4 & 7 & 7 \\
\hline Cadmium, unfiltered recoverable $(\mu \mathrm{g} / \mathrm{L})$ & 83 & 6 & .11 & 2.0 & 1.8 \\
\hline Cadmium, filtered $(\mu \mathrm{g} / \mathrm{L})$ & 83 & 6.2 & .09 & 1.5 & 1.2 \\
\hline Copper, unfiltered recoverable $(\mu \mathrm{g} / \mathrm{L})$ & 83 & 550 & 13.5 & 116 & 82 \\
\hline Copper, filtered $(\mu \mathrm{g} / \mathrm{L})$ & 83 & 303 & 4.5 & 50 & 32 \\
\hline Iron, unfiltered recoverable $(\mu \mathrm{g} / \mathrm{L})$ & 83 & 7,400 & 90 & 1,100 & 700 \\
\hline Iron, filtered $(\mu \mathrm{g} / \mathrm{L})$ & 83 & 270 & E10 & ${ }^{2} 86$ & 74 \\
\hline Lead, unfiltered recoverable $(\mu \mathrm{g} / \mathrm{L})$ & 83 & 250 & .65 & 18 & 7 \\
\hline Lead, filtered $(\mu \mathrm{g} / \mathrm{L})$ & 83 & 2.4 & $<.5$ & ${ }^{2} .6$ & .12 \\
\hline Manganese, unfiltered recoverable $(\mu \mathrm{g} / \mathrm{L})$ & 83 & 1,600 & 26 & 514 & 470 \\
\hline Manganese, filtered ( $\mu \mathrm{g} / \mathrm{L})$ & 83 & 1,700 & 21.4 & 463 & 394 \\
\hline Zinc, unfiltered recoverable $(\mu \mathrm{g} / \mathrm{L})$ & 83 & 2,200 & 45 & 602 & 460 \\
\hline Zinc, filtered $(\mu \mathrm{g} / \mathrm{L})$ & 83 & 2,200 & 34 & 496 & 358 \\
\hline Sediment, suspended concentration $(\mathrm{mg} / \mathrm{L})$ & 82 & 405 & 2 & 29 & 12 \\
\hline Sediment, suspended discharge (ton/d) & 82 & 70 & .09 & 3.5 & .92 \\
\hline Sediment, suspended (percent finer than $0.062 \mathrm{~mm}$ ) & 82 & 98 & 42 & 84 & 86 \\
\hline
\end{tabular}


Table 21. Statistical summary of water-quality data for the upper Clark Fork basin, Montana, March 1985 through September 2003-Continued

\begin{tabular}{|c|c|c|c|c|c|}
\hline Property or constituent and reporting unit & $\begin{array}{c}\text { Number of } \\
\text { samples }\end{array}$ & Maximum & Minimum & Mean & Median \\
\hline \multicolumn{6}{|c|}{ 12323600-SILVER BOW CREEK AT OPPORTUNITY, MONT. } \\
\hline Streamflow, instantaneous $\left(\mathrm{ft}^{3} / \mathrm{s}\right)$ & 86 & 361 & 13 & 77 & 52 \\
\hline Specific conductance, onsite $(\mu \mathrm{S} / \mathrm{cm})$ & 85 & 616 & 202 & 400 & 388 \\
\hline Temperature, water $\left({ }^{\circ} \mathrm{C}\right)$ & 85 & 22.5 & 0.0 & 9.3 & 9.5 \\
\hline $\mathrm{pH}$, onsite (standard units) & 85 & 9.5 & 7.2 & 8.4 & 8.4 \\
\hline Hardness, filtered (mg/L as $\mathrm{CaCO}_{3}$ ) & 85 & 220 & 60 & 143 & 140 \\
\hline Calcium, filtered $(\mathrm{mg} / \mathrm{L})$ & 85 & 62.7 & 18.5 & 42 & 41 \\
\hline Magnesium, filtered $(\mathrm{mg} / \mathrm{L})$ & 85 & 15.0 & 3.42 & 9.1 & 8.7 \\
\hline Arsenic, unfiltered recoverable $(\mu \mathrm{g} / \mathrm{L})$ & 85 & 235 & 11 & 30 & 18 \\
\hline Arsenic, filtered $(\mu \mathrm{g} / \mathrm{L})$ & 85 & 34 & 1 & 11 & 10 \\
\hline Cadmium, unfiltered recoverable $(\mu \mathrm{g} / \mathrm{L})$ & 85 & 49 & .52 & ${ }^{2} 2.6$ & 1.8 \\
\hline Cadmium, filtered $(\mu \mathrm{g} / \mathrm{L})$ & 85 & 41 & .1 & 1.5 & .9 \\
\hline Copper, unfiltered recoverable $(\mu \mathrm{g} / \mathrm{L})$ & 85 & 3,900 & 54.3 & 258 & 140 \\
\hline Copper, filtered $(\mu \mathrm{g} / \mathrm{L})$ & 85 & 450 & 19.4 & 55 & 43 \\
\hline Iron, unfiltered recoverable $(\mu \mathrm{g} / \mathrm{L})$ & 85 & 24,100 & 260 & 1,770 & 830 \\
\hline Iron, filtered $(\mu \mathrm{g} / \mathrm{L})$ & 85 & 307 & 3 & ${ }^{2} 48$ & 26 \\
\hline Lead, unfiltered recoverable $(\mu \mathrm{g} / \mathrm{L})$ & 85 & 650 & 5.38 & 47 & 16 \\
\hline Lead, filtered $(\mu \mathrm{g} / \mathrm{L})$ & 85 & 5.1 & $<.5$ & ${ }^{2} .8$ & $<1$ \\
\hline Manganese, unfiltered recoverable $(\mu \mathrm{g} / \mathrm{L})$ & 85 & 10,000 & 117 & 672 & 490 \\
\hline Manganese, filtered $(\mu \mathrm{g} / \mathrm{L})$ & 85 & 9,300 & 68 & 545 & 421 \\
\hline Zinc, unfiltered recoverable $(\mu \mathrm{g} / \mathrm{L})$ & 85 & 15,000 & 97 & 671 & 460 \\
\hline Zinc, filtered $(\mu \mathrm{g} / \mathrm{L})$ & 85 & 13,000 & 27 & 396 & 220 \\
\hline Sediment, suspended concentration $(\mathrm{mg} / \mathrm{L}$ ) & 86 & 801 & 5 & 58 & 18 \\
\hline Sediment, suspended discharge (ton/d) & 86 & 781 & .18 & 26 & 3.0 \\
\hline Sediment, suspended (percent finer than $0.062 \mathrm{~mm}$ ) & 86 & 95 & 37 & 78 & 82 \\
\hline
\end{tabular}


66 Water-Quality, Bed-Sediment, and Biological Data (October 2002 through September 2003) and Statistical Summaries of Data for Streams in the Upper Clark Fork Basin, Montana

Table 21. Statistical summary of water-quality data for the upper Clark Fork basin, Montana, March 1985 through September 2003 - Continued

\begin{tabular}{|c|c|c|c|c|c|}
\hline Property or constituent and reporting unit & $\begin{array}{c}\text { Number of } \\
\text { samples }\end{array}$ & Maximum & Minimum & Mean & Median \\
\hline \multicolumn{6}{|c|}{12323700 --MILL CREEK AT OPPORTUNITY, MONT. } \\
\hline \multicolumn{6}{|c|}{ Period of record for water-quality data: March 2003-September 2003} \\
\hline Streamflow, instantaneous $\left(\mathrm{ft}^{3} / \mathrm{s}\right)$ & 8 & 261 & 2.1 & 63 & 18 \\
\hline Specific conductance, onsite $(\mu \mathrm{S} / \mathrm{cm})$ & 8 & 207 & 59 & 139 & 149 \\
\hline Temperature, water $\left({ }^{\circ} \mathrm{C}\right)$ & 8 & 18.5 & 3.0 & 9.5 & 9.2 \\
\hline $\mathrm{pH}$, onsite (standard units) & 8 & 8.2 & 7.8 & 8.0 & 8.0 \\
\hline Hardness, filtered $\left(\mathrm{mg} / \mathrm{L}\right.$ as $\left.\mathrm{CaCO}_{3}\right)$ & 8 & 85 & 24 & 58 & 62 \\
\hline Calcium, filtered $(\mathrm{mg} / \mathrm{L})$ & 8 & 23.5 & 7.01 & 16.1 & 17.2 \\
\hline Magnesium, filtered (mg/L) & 8 & 6.57 & 1.57 & 4.25 & 4.55 \\
\hline Arsenic, unfiltered recoverable $(\mu \mathrm{g} / \mathrm{L})$ & 8 & 50 & 22 & 36 & 36 \\
\hline Arsenic, filtered $(\mu \mathrm{g} / \mathrm{L})$ & 8 & 37.0 & 20.5 & 29.8 & 30.7 \\
\hline Cadmium, unfiltered recoverable $(\mu \mathrm{g} / \mathrm{L})$ & 8 & .85 & .06 & .25 & .12 \\
\hline Cadmium, filtered $(\mu \mathrm{g} / \mathrm{L})$ & 8 & .10 & .05 & .07 & .08 \\
\hline Copper, unfiltered recoverable $(\mu \mathrm{g} / \mathrm{L})$ & 8 & 38.8 & 3.2 & 12.2 & 7.2 \\
\hline Copper, filtered $(\mu \mathrm{g} / \mathrm{L})$ & 8 & 6.0 & 2.2 & 4.2 & 4.2 \\
\hline Iron, unfiltered recoverable $(\mu \mathrm{g} / \mathrm{L})$ & 8 & 1,960 & 100 & 539 & 205 \\
\hline Iron, filtered $(\mu \mathrm{g} / \mathrm{L})$ & 8 & 60 & 37 & 47 & 48 \\
\hline Lead, unfiltered recoverable $(\mu \mathrm{g} / \mathrm{L})$ & 8 & 12.7 & .17 & 3.21 & 1.14 \\
\hline Lead, filtered $(\mu \mathrm{g} / \mathrm{L})$ & 8 & .32 & .11 & .20 & .20 \\
\hline Manganese, unfiltered recoverable $(\mu \mathrm{g} / \mathrm{L})$ & 8 & 113 & 6 & 31 & 13 \\
\hline Manganese, filtered $(\mu \mathrm{g} / \mathrm{L})$ & 8 & 15.7 & 3.6 & 7.8 & 6.7 \\
\hline Zinc, unfiltered recoverable $(\mu \mathrm{g} / \mathrm{L})$ & 8 & 41 & 2 & 13 & 8 \\
\hline Zinc, filtered $(\mu \mathrm{g} / \mathrm{L})$ & 8 & 6 & 2 & 3 & 3 \\
\hline Sediment, suspended concentration (mg/L) & 8 & 107 & 1 & 26 & 6 \\
\hline Sediment, suspended discharge (ton/d) & 8 & 55 & .01 & 11 & .26 \\
\hline Sediment, suspended (percent finer than $0.062 \mathrm{~mm}$ ) & 8 & 88 & 49 & 70 & 78 \\
\hline
\end{tabular}


Table 21. Statistical summary of water-quality data for the upper Clark Fork basin, Montana, March 1985 through September 2003-Continued

\begin{tabular}{|c|c|c|c|c|c|}
\hline Property or constituent and reporting unit & $\begin{array}{c}\text { Number of } \\
\text { samples }\end{array}$ & Maximum & Minimum & Mean & Median \\
\hline \multicolumn{6}{|c|}{$\begin{array}{l}\text { 12323720--WILLOW CREEK AT OPPORTUNITY, MONT. } \\
\text { Period of record for water quality data: March 2003-September } 2003\end{array}$} \\
\hline Streamflow, instantaneous $\left(\mathrm{ft}^{3} / \mathrm{s}\right)$ & 8 & 46 & 6.3 & 19 & 14 \\
\hline Specific conductance, onsite $(\mu \mathrm{S} / \mathrm{cm})$ & 8 & 333 & 181 & 270 & 284 \\
\hline Temperature, water $\left({ }^{\circ} \mathrm{C}\right)$ & 8 & 17.0 & 3.5 & 10.4 & 11.5 \\
\hline $\mathrm{pH}$, onsite (standard units) & 8 & 8.3 & 7.9 & 8.0 & 8.0 \\
\hline Hardness, filtered $\left(\mathrm{mg} / \mathrm{L}\right.$ as $\left.\mathrm{CaCO}_{3}\right)$ & 8 & 150 & 73 & 116 & 120 \\
\hline Calcium, filtered $(\mathrm{mg} / \mathrm{L})$ & 8 & 42.6 & 22.0 & 33.3 & 34.4 \\
\hline Magnesium, filtered (mg/L) & 8 & 9.89 & 4.37 & 7.88 & 8.54 \\
\hline Arsenic, unfiltered recoverable $(\mu \mathrm{g} / \mathrm{L})$ & 8 & 104 & 16 & 48 & 40 \\
\hline Arsenic, filtered $(\mu \mathrm{g} / \mathrm{L})$ & 8 & 101 & 13.2 & 45.8 & 37.8 \\
\hline Cadmium, unfiltered recoverable $(\mu \mathrm{g} / \mathrm{L})$ & 8 & .30 & .04 & .11 & .10 \\
\hline Cadmium, filtered $(\mu \mathrm{g} / \mathrm{L})$ & 8 & .08 & E. 03 & .05 & .05 \\
\hline Copper, unfiltered recoverable $(\mu \mathrm{g} / \mathrm{L})$ & 8 & 36.9 & 4.4 & 14.0 & 11.2 \\
\hline Copper, filtered $(\mu \mathrm{g} / \mathrm{L})$ & 8 & 14.1 & 2.4 & 6.3 & 5.0 \\
\hline Iron, unfiltered recoverable $(\mu \mathrm{g} / \mathrm{L})$ & 8 & 840 & 80 & 300 & 235 \\
\hline Iron, filtered $(\mu \mathrm{g} / \mathrm{L})$ & 8 & 87 & 8 & 45 & 48 \\
\hline Lead, unfiltered recoverable $(\mu \mathrm{g} / \mathrm{L})$ & 8 & 8.41 & .71 & 2.62 & 2.05 \\
\hline Lead, filtered $(\mu \mathrm{g} / \mathrm{L})$ & 8 & .50 & .06 & .26 & .26 \\
\hline Manganese, unfiltered recoverable $(\mu \mathrm{g} / \mathrm{L})$ & 8 & 70 & 14 & 38 & 34 \\
\hline Manganese, filtered $(\mu \mathrm{g} / \mathrm{L})$ & 8 & 49.3 & 10.6 & 25.7 & 21.6 \\
\hline Zinc, unfiltered recoverable $(\mu \mathrm{g} / \mathrm{L})$ & 8 & 41 & 4 & 15 & 14 \\
\hline Zinc, filtered $(\mu \mathrm{g} / \mathrm{L})$ & 8 & 10 & 2 & 6 & 6 \\
\hline Sediment, suspended concentration $(\mathrm{mg} / \mathrm{L})$ & 8 & 51 & 2 & 14 & 8 \\
\hline Sediment, suspended discharge (ton/d) & 8 & 6.3 & .04 & 1.2 & .28 \\
\hline Sediment, suspended (percent finer than $0.062 \mathrm{~mm}$ ) & 8 & 96 & 76 & 88 & 90 \\
\hline
\end{tabular}


Table 21. Statistical summary of water-quality data for the upper Clark Fork basin, Montana, March 1985 through September 2003-Continued

\begin{tabular}{|c|c|c|c|c|c|}
\hline Property or constituent and reporting unit & $\begin{array}{l}\text { Number of } \\
\text { samples }\end{array}$ & Maximum & Minimum & Mean & Median \\
\hline \multicolumn{6}{|c|}{$\begin{array}{l}\text { 12323750--SILVER BOW CREEK AT WARM SPRINGS, MONT. } \\
\text { Period of record for water-quality data: March 1993-September } 2003\end{array}$} \\
\hline Streamflow, instantaneous $\left(\mathrm{ft}^{3} / \mathrm{s}\right)$ & 92 & 662 & 16 & 148 & 105 \\
\hline Specific conductance, onsite $(\mu \mathrm{S} / \mathrm{cm})$ & 90 & 783 & 249 & 460 & 466 \\
\hline Temperature, water $\left({ }^{\circ} \mathrm{C}\right)$ & 91 & 25.0 & .5 & 11.2 & 11.5 \\
\hline $\mathrm{pH}$, onsite (standard units) & 90 & 9.3 & 8.0 & 8.8 & 8.8 \\
\hline Hardness, filtered (mg/L as $\mathrm{CaCO}_{3}$ ) & 90 & 310 & 97 & 190 & 190 \\
\hline Calcium, filtered (mg/L) & 90 & 90.4 & 27.9 & 55 & 56 \\
\hline Magnesium, filtered (mg/L) & 90 & 21.4 & 5.94 & 13 & 12 \\
\hline Arsenic, unfiltered recoverable $(\mu \mathrm{g} / \mathrm{L})$ & 90 & 94 & 10 & 26 & 24 \\
\hline Arsenic, filtered $(\mu \mathrm{g} / \mathrm{L})$ & 90 & 60 & 6.8 & 22 & 21 \\
\hline Cadmium, unfiltered recoverable $(\mu \mathrm{g} / \mathrm{L})$ & 90 & .56 & $<.1$ & ${ }^{2}<.12$ & $<1$ \\
\hline Cadmium, filtered $(\mu \mathrm{g} / \mathrm{L})$ & 90 & .3 & $<.1$ & ${ }^{2} .08$ & $<.1$ \\
\hline Copper, unfiltered recoverable $(\mu \mathrm{g} / \mathrm{L})$ & 90 & 96.8 & 3.4 & 20 & 14 \\
\hline Copper, filtered $(\mu \mathrm{g} / \mathrm{L})$ & 90 & 40 & 1.9 & 10 & 8 \\
\hline Iron, unfiltered recoverable $(\mu \mathrm{g} / \mathrm{L})$ & 90 & 3,000 & 70 & 376 & 300 \\
\hline Iron, filtered $(\mu \mathrm{g} / \mathrm{L})$ & 90 & 93 & $<5$ & ${ }^{2} 17$ & 15 \\
\hline Lead, unfiltered recoverable $(\mu \mathrm{g} / \mathrm{L})$ & 90 & 41.8 & $<1$ & $2_{3}$ & 2 \\
\hline Lead, filtered $(\mu \mathrm{g} / \mathrm{L})$ & 90 & 1.0 & $<.08$ & 2.1 & $<.5$ \\
\hline Manganese, unfiltered recoverable $(\mu \mathrm{g} / \mathrm{L})$ & 90 & 899 & 55 & 194 & 150 \\
\hline Manganese, filtered $(\mu \mathrm{g} / \mathrm{L})$ & 90 & 875 & 11.8 & 126 & 80 \\
\hline Zinc, unfiltered recoverable $(\mu \mathrm{g} / \mathrm{L})$ & 90 & 180 & $<10$ & 242 & 30 \\
\hline Zinc, filtered $(\mu \mathrm{g} / \mathrm{L})$ & 90 & 73 & $<1$ & ${ }^{2} 10$ & 6 \\
\hline Sediment, suspended concentration $(\mathrm{mg} / \mathrm{L})$ & 92 & 229 & 1 & 13 & 7 \\
\hline Sediment, suspended discharge (ton/d) & 92 & 279 & .11 & 8.7 & 1.8 \\
\hline Sediment, suspended (percent finer than $0.062 \mathrm{~mm}$ ) & 91 & 97 & 43 & 82 & 85 \\
\hline
\end{tabular}


Table 21. Statistical summary of water-quality data for the upper Clark Fork basin, Montana, March 1985 through September 2003-Continued

\begin{tabular}{|c|c|c|c|c|c|}
\hline Property or constituent and reporting unit & $\begin{array}{c}\text { Number of } \\
\text { samples }\end{array}$ & Maximum & Minimum & Mean & Median \\
\hline \multicolumn{6}{|c|}{ 12323770--WARM SPRINGS CREEK AT WARM SPRINGS, MONT. } \\
\hline Streamflow, instantaneous $\left(\mathrm{ft}^{3} / \mathrm{s}\right)$ & 66 & 420 & 2.8 & 101 & 57 \\
\hline Specific conductance, onsite $(\mu \mathrm{S} / \mathrm{cm})$ & 65 & 795 & 139 & 297 & 283 \\
\hline Temperature, water $\left({ }^{\circ} \mathrm{C}\right)$ & 66 & 20.0 & .5 & 9.3 & 9.2 \\
\hline $\mathrm{pH}$, onsite (standard units) & 65 & 8.7 & 7.4 & 8.3 & 8.3 \\
\hline Hardness, filtered $\left(\mathrm{mg} / \mathrm{L}\right.$ as $\left.\mathrm{CaCO}_{3}\right)$ & 65 & 420 & 40 & 143 & 130 \\
\hline Calcium, filtered (mg/L) & 65 & 130 & 10.5 & 44 & 40 \\
\hline Magnesium, filtered (mg/L) & 65 & 22.0 & 3.29 & 8.3 & 7.5 \\
\hline Arsenic, unfiltered recoverable $(\mu \mathrm{g} / \mathrm{L})$ & 65 & 27 & 3 & 8 & 6 \\
\hline Arsenic, filtered $(\mu \mathrm{g} / \mathrm{L})$ & 65 & 14 & 2 & 5 & 5 \\
\hline Cadmium, unfiltered recoverable $(\mu \mathrm{g} / \mathrm{L})$ & 65 & .36 & $<.1$ & ${ }^{2} .08$ & $<1$ \\
\hline Cadmium, filtered $(\mu \mathrm{g} / \mathrm{L})$ & 65 & E.1 & $<.1$ & ${ }^{2} .04$ & $<.1$ \\
\hline Copper, unfiltered recoverable $(\mu \mathrm{g} / \mathrm{L})$ & 65 & 96.7 & 2.3 & 21 & 10 \\
\hline Copper, filtered $(\mu \mathrm{g} / \mathrm{L})$ & 65 & 16 & 1 & 4 & 3 \\
\hline Iron, unfiltered recoverable $(\mu \mathrm{g} / \mathrm{L})$ & 65 & 1,670 & 40 & 326 & 120 \\
\hline Iron, filtered $(\mu \mathrm{g} / \mathrm{L})$ & 65 & 30 & $<5$ & ${ }^{2} 11$ & 9 \\
\hline Lead, unfiltered recoverable $(\mu \mathrm{g} / \mathrm{L})$ & 65 & 14 & $<1$ & ${ }^{2} 2$ & .4 \\
\hline Lead, filtered $(\mu \mathrm{g} / \mathrm{L})$ & 65 & 1.8 & $<.08$ & 2.1 & $<.5$ \\
\hline Manganese, unfiltered recoverable $(\mu \mathrm{g} / \mathrm{L})$ & 65 & 1,400 & 57 & 228 & 190 \\
\hline Manganese, filtered $(\mu \mathrm{g} / \mathrm{L})$ & 65 & 570 & 22.6 & 135 & 110 \\
\hline Zinc, unfiltered recoverable $(\mu \mathrm{g} / \mathrm{L})$ & 65 & 60 & $<10$ & ${ }^{2} 11$ & 3 \\
\hline Zinc, filtered $(\mu \mathrm{g} / \mathrm{L})$ & 65 & 10 & $<1$ & ${ }^{2} 2$ & $<20$ \\
\hline Sediment, suspended concentration $(\mathrm{mg} / \mathrm{L})$ & 66 & 100 & 2 & 19 & 8 \\
\hline Sediment, suspended discharge (ton/d) & 66 & 87 & .05 & 10 & 1.2 \\
\hline Sediment, suspended (percent finer than $0.062 \mathrm{~mm}$ ) & 66 & 88 & 55 & 73 & 74 \\
\hline
\end{tabular}


70 Water-Quality, Bed-Sediment, and Biological Data (October 2002 through September 2003) and Statistical Summaries of Data for Streams in the Upper Clark Fork Basin, Montana

Table 21. Statistical summary of water-quality data for the upper Clark Fork basin, Montana, March 1985 through September 2003-Continued

\begin{tabular}{|c|c|c|c|c|c|}
\hline Property or constituent and reporting unit & $\begin{array}{c}\text { Number of } \\
\text { samples }\end{array}$ & Maximum & Minimum & Mean & Median \\
\hline \multicolumn{6}{|c|}{ 12323800--CLARK FORK NEAR GALEN, MONT. } \\
\hline \multicolumn{6}{|c|}{ Period of record for water-quality data: July 1988-September 2003} \\
\hline Streamflow, instantaneous $\left(\mathrm{ft}^{3} / \mathrm{s}\right)$ & 133 & 1,050 & 14 & 215 & 129 \\
\hline Specific conductance, onsite $(\mu \mathrm{S} / \mathrm{cm})$ & 121 & 720 & 197 & 425 & 435 \\
\hline Temperature, water $\left({ }^{\circ} \mathrm{C}\right)$ & 132 & 22.5 & 0.0 & 10.0 & 10.0 \\
\hline pH, onsite (standard units) & 120 & 9.0 & 7.5 & 8.5 & 8.5 \\
\hline Hardness, filtered $\left(\mathrm{mg} / \mathrm{L}\right.$ as $\left.\mathrm{CaCO}_{3}\right)$ & 119 & 370 & 81 & 185 & 190 \\
\hline Calcium, filtered (mg/L) & 119 & 110 & 24.2 & 55 & 57 \\
\hline Magnesium, filtered (mg/L) & 119 & 22.0 & 5.08 & 12 & 12 \\
\hline Arsenic, unfiltered recoverable $(\mu \mathrm{g} / \mathrm{L})$ & 119 & 78 & 3 & 20 & 17 \\
\hline Arsenic, filtered $(\mu \mathrm{g} / \mathrm{L})$ & 119 & 53 & 4 & 15 & 13 \\
\hline Cadmium, unfiltered recoverable $(\mu \mathrm{g} / \mathrm{L})$ & 119 & 3 & $<.1$ & ${ }^{2} .2$ & $<1$ \\
\hline Cadmium, filtered $(\mu \mathrm{g} / \mathrm{L})$ & 119 & 1 & $<.1$ & ${ }^{2} .07$ & $<.1$ \\
\hline Copper, unfiltered recoverable $(\mu \mathrm{g} / \mathrm{L})$ & 118 & 240 & 4.8 & 32 & 19 \\
\hline Copper, filtered $(\mu \mathrm{g} / \mathrm{L})$ & 119 & 50 & 2.3 & 10 & 8 \\
\hline Iron, unfiltered recoverable $(\mu \mathrm{g} / \mathrm{L})$ & 119 & 9,200 & 60 & 548 & 290 \\
\hline Iron, filtered $(\mu \mathrm{g} / \mathrm{L})$ & 119 & 110 & $<3$ & ${ }^{2} 16$ & 11 \\
\hline Lead, unfiltered recoverable $(\mu \mathrm{g} / \mathrm{L})$ & 119 & 31.0 & $<1$ & 24 & 2 \\
\hline Lead, filtered $(\mu \mathrm{g} / \mathrm{L})$ & 119 & 3 & $<.08$ & ${ }^{2} .2$ & $<1$ \\
\hline Manganese, unfiltered recoverable $(\mu \mathrm{g} / \mathrm{L})$ & 119 & 1,400 & 47 & 253 & 200 \\
\hline Manganese, filtered $(\mu \mathrm{g} / \mathrm{L})$ & 119 & 460 & 25.2 & 116 & 82 \\
\hline Zinc, unfiltered recoverable $(\mu \mathrm{g} / \mathrm{L})$ & 119 & 360 & $<10$ & 248 & 30 \\
\hline Zinc, filtered $(\mu \mathrm{g} / \mathrm{L})$ & 119 & 110 & $<1$ & ${ }^{2} 12$ & 7 \\
\hline Sediment, suspended concentration $(\mathrm{mg} / \mathrm{L}$ ) & 133 & 338 & 2 & 20 & 9 \\
\hline Sediment, suspended discharge (ton/d) & 133 & 459 & .12 & 23 & 2.8 \\
\hline Sediment, suspended (percent finer than $0.062 \mathrm{~mm}$ ) & 132 & 97 & 41 & 78 & 78 \\
\hline
\end{tabular}


TABLE 21

Table 21. Statistical summary of water-quality data for the upper Clark Fork basin, Montana, March 1985 through September 2003-Continued

\begin{tabular}{|c|c|c|c|c|c|}
\hline Property or constituent and reporting unit & $\begin{array}{c}\text { Number of } \\
\text { samples }\end{array}$ & Maximum & Minimum & Mean & Median \\
\hline \multicolumn{6}{|c|}{ 12323850--LOST CREEK NEAR GALEN, MONT. } \\
\hline \multicolumn{6}{|c|}{ Period of record for water-quality data: March 2003-September 2003} \\
\hline Streamflow, instantaneous $\left(\mathrm{ft}^{3} / \mathrm{s}\right)$ & 8 & 59 & 1.3 & 20 & 14 \\
\hline Specific conductance, onsite $(\mu \mathrm{S} / \mathrm{cm})$ & 8 & 861 & 540 & 633 & 626 \\
\hline Temperature, water $\left({ }^{\circ} \mathrm{C}\right)$ & 8 & 26.5 & 4.5 & 15.6 & 16.2 \\
\hline $\mathrm{pH}$, onsite (standard units) & 8 & 8.7 & 8.4 & 8.6 & 8.6 \\
\hline Hardness, filtered (mg/L as $\left.\mathrm{CaCO}_{3}\right)$ & 8 & 420 & 200 & 296 & 290 \\
\hline Calcium, filtered (mg/L) & 8 & 117 & 48.5 & 83.2 & 82.0 \\
\hline Magnesium, filtered (mg/L) & 8 & 32.3 & 18.0 & 21.7 & 20.6 \\
\hline Arsenic, unfiltered recoverable $(\mu \mathrm{g} / \mathrm{L})$ & 8 & 43 & 9 & 18 & 15 \\
\hline Arsenic, filtered $(\mu \mathrm{g} / \mathrm{L})$ & 8 & 41.8 & 10.3 & 17.2 & 14.0 \\
\hline Cadmium, unfiltered recoverable $(\mu \mathrm{g} / \mathrm{L})$ & 8 & .11 & E.02 & .05 & .04 \\
\hline Cadmium, filtered $(\mu \mathrm{g} / \mathrm{L})$ & 8 & .05 & E.02 & .03 & .03 \\
\hline Copper, unfiltered recoverable $(\mu \mathrm{g} / \mathrm{L})$ & 8 & 22.5 & 4.4 & 8.6 & 6.3 \\
\hline Copper, filtered $(\mu \mathrm{g} / \mathrm{L})$ & 8 & 6.7 & 2.5 & 3.5 & 3.1 \\
\hline Iron, unfiltered recoverable $(\mu \mathrm{g} / \mathrm{L})$ & 8 & 280 & 10 & 105 & 70 \\
\hline Iron, filtered $(\mu \mathrm{g} / \mathrm{L})$ & 8 & 18 & $<8$ & ${ }^{2} 7$ & 6 \\
\hline Lead, unfiltered recoverable $(\mu \mathrm{g} / \mathrm{L})$ & 8 & 1.30 & E.04 & .46 & .31 \\
\hline Lead, filtered $(\mu \mathrm{g} / \mathrm{L})$ & 8 & .33 & $<.08$ & -- & $<.08$ \\
\hline Manganese, unfiltered recoverable $(\mu \mathrm{g} / \mathrm{L})$ & 8 & 33 & 2 & 15 & 13 \\
\hline Manganese, filtered $(\mu \mathrm{g} / \mathrm{L})$ & 8 & 23.6 & 1.9 & 10.4 & 8.6 \\
\hline Zinc, unfiltered recoverable $(\mu \mathrm{g} / \mathrm{L})$ & 8 & 9 & $<2$ & 24 & 3 \\
\hline Zinc, filtered $(\mu \mathrm{g} / \mathrm{L})$ & 8 & 2 & $<1$ & -- & $<1$ \\
\hline Sediment, suspended concentration (mg/L) & 8 & 24 & 2 & 11 & 9 \\
\hline Sediment, suspended discharge (ton/d) & 8 & 3.8 & .01 & .89 & .28 \\
\hline Sediment, suspended (percent finer than $0.062 \mathrm{~mm}$ ) & 8 & 77 & 18 & 53 & 55 \\
\hline
\end{tabular}


72 Water-Quality, Bed-Sediment, and Biological Data (October 2002 through September 2003) and Statistical Summaries of Data for Streams in the Upper Clark Fork Basin, Montana

Table 21. Statistical summary of water-quality data for the upper Clark Fork basin, Montana, March 1985 through September 2003-Continued

\begin{tabular}{|c|c|c|c|c|c|}
\hline Property or constituent and reporting unit & $\begin{array}{l}\text { Number of } \\
\text { samples }\end{array}$ & Maximum & Minimum & Mean & Median \\
\hline \multicolumn{6}{|c|}{$\begin{array}{l}\text { 12324200--CLARK FORK AT DEER LODGE, MONT. } \\
\text { Period of record for water-quality data: March 1985-September } 2003\end{array}$} \\
\hline Streamflow, instantaneous $\left(\mathrm{ft}^{3} / \mathrm{s}\right)$ & 185 & 1,920 & 23 & 296 & 225 \\
\hline Specific conductance, onsite $(\mu \mathrm{S} / \mathrm{cm})$ & 168 & 642 & 234 & 485 & 506 \\
\hline Temperature, water $\left({ }^{\circ} \mathrm{C}\right)$ & 184 & 23.0 & 0.0 & 9.8 & 10.0 \\
\hline pH, onsite (standard units) & 133 & 8.9 & 7.4 & 8.3 & 8.3 \\
\hline Hardness, filtered $\left(\mathrm{mg} / \mathrm{L}\right.$ as $\left.\mathrm{CaCO}_{3}\right)$ & 125 & 270 & 95 & 204 & 210 \\
\hline Calcium, filtered $(\mathrm{mg} / \mathrm{L})$ & 125 & 81.0 & 28.2 & 60 & 63 \\
\hline Magnesium, filtered (mg/L) & 125 & 18 & 5.9 & 13 & 14 \\
\hline Arsenic, unfiltered recoverable $(\mu \mathrm{g} / \mathrm{L})$ & 135 & 215 & 8 & 25 & 18 \\
\hline Arsenic, filtered $(\mu \mathrm{g} / \mathrm{L})$ & 135 & 39 & 6 & 14 & 13 \\
\hline Cadmium, unfiltered recoverable $(\mu \mathrm{g} / \mathrm{L})$ & 135 & 5 & $<.1$ & 2.5 & $<1$ \\
\hline Cadmium, filtered $(\mu \mathrm{g} / \mathrm{L})$ & 135 & 2 & $<.1$ & ${ }^{2} .09$ & $<1$ \\
\hline Copper, unfiltered recoverable $(\mu \mathrm{g} / \mathrm{L})$ & 134 & 1,500 & 8.2 & 94 & 41 \\
\hline Copper, filtered $(\mu \mathrm{g} / \mathrm{L})$ & 135 & 120 & 3.2 & 12 & 9 \\
\hline Iron, unfiltered recoverable $(\mu \mathrm{g} / \mathrm{L})$ & 135 & 29,000 & 30 & 1,770 & 660 \\
\hline Iron, filtered $(\mu \mathrm{g} / \mathrm{L})$ & 135 & 190 & $<3$ & ${ }^{2} 15$ & 9 \\
\hline Lead, unfiltered recoverable $(\mu \mathrm{g} / \mathrm{L})$ & 135 & 200 & $<1$ & 212 & 5 \\
\hline Lead, filtered $(\mu \mathrm{g} / \mathrm{L})$ & 135 & 6 & $<.08$ & 2.4 & $<1$ \\
\hline Manganese, unfiltered recoverable $(\mu \mathrm{g} / \mathrm{L})$ & 135 & 4,600 & 12 & 277 & 160 \\
\hline Manganese, filtered $(\mu \mathrm{g} / \mathrm{L})$ & 135 & 400 & 1 & 44 & 33 \\
\hline Zinc, unfiltered recoverable $(\mu \mathrm{g} / \mathrm{L})$ & 135 & 1,700 & 4 & 104 & 53 \\
\hline Zinc, filtered $(\mu \mathrm{g} / \mathrm{L})$ & 135 & 230 & $<10$ & 214 & 10 \\
\hline Sediment, suspended concentration $(\mathrm{mg} / \mathrm{L})$ & 185 & 2,250 & 2 & 79 & 23 \\
\hline Sediment, suspended discharge (ton/d) & 185 & 8,690 & .29 & 175 & 12 \\
\hline Sediment, suspended (percent finer than $0.062 \mathrm{~mm}$ ) & 176 & 99 & 40 & 71 & 72 \\
\hline
\end{tabular}


Table 21. Statistical summary of water-quality data for the upper Clark Fork basin, Montana, March 1985 through September 2003-Continued

\begin{tabular}{|c|c|c|c|c|c|}
\hline Property or constituent and reporting unit & $\begin{array}{c}\text { Number of } \\
\text { samples }\end{array}$ & Maximum & Minimum & Mean & Median \\
\hline \multicolumn{6}{|c|}{ 12324590--LITTLE BLACKFOOT RIVER NEAR GARRISON, MONT. } \\
\hline \multicolumn{6}{|c|}{ Period of record for water-quality data: March 1985-September 2003} \\
\hline Streamflow, instantaneous $\left(\mathrm{ft}^{3} / \mathrm{s}\right)$ & 104 & 2,080 & 15 & 283 & 178 \\
\hline Specific conductance, onsite $(\mu \mathrm{S} / \mathrm{cm})$ & 92 & 347 & 120 & 231 & 222 \\
\hline Temperature, water $\left({ }^{\circ} \mathrm{C}\right)$ & 103 & 22 & 0.0 & 8.5 & 9.0 \\
\hline pH, onsite (standard units) & 91 & 8.6 & 7.0 & 8.1 & 8.1 \\
\hline Hardness, filtered (mg/L as $\left.\mathrm{CaCO}_{3}\right)$ & 86 & 170 & 51 & 106 & 100 \\
\hline Calcium, filtered (mg/L) & 86 & 49.3 & 14.0 & 31 & 30 \\
\hline Magnesium, filtered (mg/L) & 86 & 11.9 & 3.30 & 7.1 & 7.0 \\
\hline Arsenic, unfiltered recoverable $(\mu \mathrm{g} / \mathrm{L})$ & 91 & 17 & 4 & 7 & 6 \\
\hline Arsenic, filtered $(\mu \mathrm{g} / \mathrm{L})$ & 91 & 7 & 3 & 5 & 5 \\
\hline Cadmium, unfiltered recoverable $(\mu \mathrm{g} / \mathrm{L})$ & 91 & 2 & $<.04$ & 2.1 & $<1$ \\
\hline Cadmium, filtered $(\mu \mathrm{g} / \mathrm{L})$ & 91 & .2 & $<.04$ & -- & $<.1$ \\
\hline Copper, unfiltered recoverable $(\mu \mathrm{g} / \mathrm{L})$ & 90 & 45 & $<1$ & ${ }^{2} 4$ & 2 \\
\hline Copper, filtered $(\mu \mathrm{g} / \mathrm{L})$ & 91 & 7 & $<1$ & ${ }^{2} 2$ & 1 \\
\hline Iron, unfiltered recoverable $(\mu \mathrm{g} / \mathrm{L})$ & 91 & 25,000 & 20 & 1,090 & 260 \\
\hline Iron, filtered $(\mu \mathrm{g} / \mathrm{L})$ & 91 & 120 & $<3$ & 235 & 23 \\
\hline Lead, unfiltered recoverable $(\mu \mathrm{g} / \mathrm{L})$ & 91 & 25 & $<1$ & ${ }^{2} 2$ & $<5$ \\
\hline Lead, filtered $(\mu \mathrm{g} / \mathrm{L})$ & 90 & 6 & $<.08$ & ${ }^{2} .4$ & $<1$ \\
\hline Manganese, unfiltered recoverable $(\mu \mathrm{g} / \mathrm{L})$ & 91 & 1,100 & $<10$ & ${ }^{2} 67$ & 30 \\
\hline Manganese, filtered $(\mu \mathrm{g} / \mathrm{L})$ & 91 & 45.2 & 1.0 & 9 & 8 \\
\hline Zinc, unfiltered recoverable $(\mu \mathrm{g} / \mathrm{L})$ & 91 & 140 & $<1$ & ${ }^{2} 12$ & $<40$ \\
\hline Zinc, filtered $(\mu \mathrm{g} / \mathrm{L})$ & 91 & 24 & $<1$ & 23 & $<20$ \\
\hline Sediment, suspended concentration (mg/L) & 104 & 1,410 & 1 & 49 & 9 \\
\hline Sediment, suspended discharge (ton/d) & 104 & 7,920 & .08 & 134 & 4.4 \\
\hline Sediment, suspended (percent finer than $0.062 \mathrm{~mm}$ ) & 104 & 97 & 32 & 75 & 80 \\
\hline
\end{tabular}


74 Water-Quality, Bed-Sediment, and Biological Data (October 2002 through September 2003) and Statistical Summaries of Data for Streams in the Upper Clark Fork Basin, Montana

Table 21. Statistical summary of water-quality data for the upper Clark Fork basin, Montana, March 1985 through September 2003-Continued

\begin{tabular}{|c|c|c|c|c|c|}
\hline Property or constituent and reporting unit & $\begin{array}{c}\text { Number of } \\
\text { samples }\end{array}$ & Maximum & Minimum & Mean & Median \\
\hline \multirow{2}{*}{\multicolumn{6}{|c|}{ 12324680--CLARK FORK AT GOLDCREEK, MONT. }} \\
\hline \multicolumn{5}{|c|}{ Period of record for water-quality data: March 1993-September 2003} & \\
\hline Streamflow, instantaneous $\left(\mathrm{ft}^{3} / \mathrm{s}\right)$ & 91 & 3,920 & 87 & 774 & 529 \\
\hline Specific conductance, onsite $(\mu \mathrm{S} / \mathrm{cm})$ & 90 & 510 & 206 & 370 & 386 \\
\hline Temperature, water $\left({ }^{\circ} \mathrm{C}\right)$ & 91 & 21.5 & 0.0 & 9.7 & 10.0 \\
\hline $\mathrm{pH}$, onsite (standard units) & 90 & 8.8 & 7.9 & 8.3 & 8.3 \\
\hline Hardness, filtered (mg/L as $\left.\mathrm{CaCO}_{3}\right)$ & 90 & 230 & 86 & 162 & 170 \\
\hline Calcium, filtered $(\mathrm{mg} / \mathrm{L})$ & 90 & 68.0 & 25.9 & 48 & 50 \\
\hline Magnesium, filtered (mg/L) & 90 & 15.0 & 5.15 & 10 & 11 \\
\hline Arsenic, unfiltered recoverable $(\mu \mathrm{g} / \mathrm{L})$ & 90 & 75 & 7 & 16 & 13 \\
\hline Arsenic, filtered $(\mu \mathrm{g} / \mathrm{L})$ & 90 & 20 & 5.8 & 10 & 10 \\
\hline Cadmium, unfiltered recoverable $(\mu \mathrm{g} / \mathrm{L})$ & 90 & 2 & $<.1$ & ${ }^{2} .2$ & $<1$ \\
\hline Cadmium, filtered $(\mu \mathrm{g} / \mathrm{L})$ & 90 & .2 & $<.1$ & ${ }^{2} .05$ & $<.1$ \\
\hline Copper, unfiltered recoverable $(\mu \mathrm{g} / \mathrm{L})$ & 89 & 440 & 5.2 & 46 & 29 \\
\hline Copper, filtered $(\mu \mathrm{g} / \mathrm{L})$ & 89 & 36 & 2.1 & 7 & 6 \\
\hline Iron, unfiltered recoverable $(\mu \mathrm{g} / \mathrm{L})$ & 90 & 12,000 & 30 & 999 & 530 \\
\hline Iron, filtered $(\mu \mathrm{g} / \mathrm{L})$ & 90 & 100 & $<3$ & ${ }^{2} 20$ & 13 \\
\hline Lead, unfiltered recoverable $(\mu \mathrm{g} / \mathrm{L})$ & 89 & 73 & $<1$ & 27 & 4 \\
\hline Lead, filtered $(\mu \mathrm{g} / \mathrm{L})$ & 89 & .8 & $<.08$ & ${ }^{2} .1$ & $<.5$ \\
\hline Manganese, unfiltered recoverable $(\mu \mathrm{g} / \mathrm{L})$ & 90 & 1,100 & 10 & 140 & 100 \\
\hline Manganese, filtered $(\mu \mathrm{g} / \mathrm{L})$ & 90 & 57.3 & 4.0 & 20 & 19 \\
\hline Zinc, unfiltered recoverable $(\mu \mathrm{g} / \mathrm{L})$ & 90 & 510 & 2 & 54 & 35 \\
\hline Zinc, filtered $(\mu \mathrm{g} / \mathrm{L})$ & 90 & 26 & $<1$ & 27 & 4 \\
\hline Sediment, suspended concentration $(\mathrm{mg} / \mathrm{L})$ & 91 & 752 & 2 & 58 & 24 \\
\hline Sediment, suspended discharge (ton/d) & 91 & 7,960 & .94 & 261 & 37 \\
\hline Sediment, suspended (percent finer than $0.062 \mathrm{~mm}$ ) & 91 & 94 & 43 & 75 & 78 \\
\hline
\end{tabular}


Table 21. Statistical summary of water-quality data for the upper Clark Fork basin, Montana, March 1985 through September 2003-Continued

\begin{tabular}{|c|c|c|c|c|c|}
\hline Property or constituent and reporting unit & $\begin{array}{c}\text { Number of } \\
\text { samples }\end{array}$ & Maximum & Minimum & Mean & Median \\
\hline \multicolumn{6}{|c|}{ 12331500--FLINT CREEK NEAR DRUMMOND, MONT. } \\
\hline \multicolumn{6}{|c|}{ Period of record for water-quality data: March 1985-September 2003} \\
\hline Streamflow, instantaneous $\left(\mathrm{ft}^{3} / \mathrm{s}\right)$ & 133 & 892 & 2.8 & 179 & 119 \\
\hline Specific conductance, onsite $(\mu \mathrm{S} / \mathrm{cm})$ & 122 & 529 & 134 & 307 & 304 \\
\hline Temperature, water $\left({ }^{\circ} \mathrm{C}\right)$ & 131 & 21.0 & 0.0 & 9.1 & 9.5 \\
\hline $\mathrm{pH}$, onsite (standard units) & 119 & 8.8 & 7.5 & 8.3 & 8.3 \\
\hline Hardness, filtered $\left(\mathrm{mg} / \mathrm{L}\right.$ as $\left.\mathrm{CaCO}_{3}\right)$ & 112 & 260 & 59 & 144 & 140 \\
\hline Calcium, filtered (mg/L) & 112 & 73.0 & 16.4 & 39 & 38 \\
\hline Magnesium, filtered (mg/L) & 112 & 20 & 4.3 & 11 & 12 \\
\hline Arsenic, unfiltered recoverable $(\mu \mathrm{g} / \mathrm{L})$ & 119 & 57 & 7 & 17 & 13 \\
\hline Arsenic, filtered $(\mu \mathrm{g} / \mathrm{L})$ & 119 & 20 & 5 & 9 & 9 \\
\hline Cadmium, unfiltered recoverable $(\mu \mathrm{g} / \mathrm{L})$ & 119 & 3 & $<.04$ & 2.2 & $<1$ \\
\hline Cadmium, filtered $(\mu \mathrm{g} / \mathrm{L})$ & 119 & .1 & $<.04$ & -- & $<.1$ \\
\hline Copper, unfiltered recoverable $(\mu \mathrm{g} / \mathrm{L})$ & 118 & 32 & 1 & 6 & 4 \\
\hline Copper, filtered $(\mu \mathrm{g} / \mathrm{L})$ & 119 & 7 & $<1$ & ${ }^{2} 2$ & 2 \\
\hline Iron, unfiltered recoverable $(\mu \mathrm{g} / \mathrm{L})$ & 119 & 7,200 & 60 & 872 & 450 \\
\hline Iron, filtered $(\mu \mathrm{g} / \mathrm{L})$ & 119 & 240 & $<3$ & 238 & 21 \\
\hline Lead, unfiltered recoverable $(\mu \mathrm{g} / \mathrm{L})$ & 119 & 87 & $<1$ & ${ }^{2} 11$ & 7 \\
\hline Lead, filtered $(\mu \mathrm{g} / \mathrm{L})$ & 119 & 7 & $<.5$ & 2.6 & $<1$ \\
\hline Manganese, unfiltered recoverable $(\mu \mathrm{g} / \mathrm{L})$ & 119 & 1,600 & 50 & 207 & 130 \\
\hline Manganese, filtered $(\mu \mathrm{g} / \mathrm{L})$ & 119 & 139 & 14 & 42 & 37 \\
\hline Zinc, unfiltered recoverable $(\mu \mathrm{g} / \mathrm{L})$ & 119 & 290 & $<10$ & 237 & 20 \\
\hline Zinc, filtered $(\mu \mathrm{g} / \mathrm{L})$ & 119 & 27 & $<1$ & ${ }^{2} 5$ & 3 \\
\hline Sediment, suspended concentration $(\mathrm{mg} / \mathrm{L})$ & 133 & 556 & 3 & 50 & 26 \\
\hline Sediment, suspended discharge (ton/d) & 133 & 904 & .02 & 44 & 7.8 \\
\hline Sediment, suspended (percent finer than $0.062 \mathrm{~mm}$ ) & 133 & 98 & 28 & 80 & 84 \\
\hline
\end{tabular}


76 Water-Quality, Bed-Sediment, and Biological Data (October 2002 through September 2003) and Statistical Summaries of Data for Streams in the Upper Clark Fork Basin, Montana

Table 21. Statistical summary of water-quality data for the upper Clark Fork basin, Montana, March 1985 through September 2003 - Continued

\begin{tabular}{|c|c|c|c|c|c|}
\hline Property or constituent and reporting unit & $\begin{array}{l}\text { Number of } \\
\text { samples }\end{array}$ & Maximum & Minimum & Mean & Median \\
\hline \multicolumn{6}{|c|}{$\begin{array}{l}\text { 12331800--CLARK FORK NEAR DRUMMOND, MONT. } \\
\text { Period of record for water-quality data: March 1993-September } 2003\end{array}$} \\
\hline Streamflow, instantaneous $\left(\mathrm{ft}^{3} / \mathrm{s}\right)$ & 91 & 3,860 & 149 & 1,080 & 817 \\
\hline Specific conductance, onsite $(\mu \mathrm{S} / \mathrm{cm})$ & 90 & 630 & 189 & 406 & 420 \\
\hline Temperature, water $\left({ }^{\circ} \mathrm{C}\right)$ & 91 & 22.5 & .5 & 10.6 & 11.0 \\
\hline $\mathrm{pH}$, onsite (standard units) & 90 & 8.5 & 7.8 & 8.3 & 8.3 \\
\hline Hardness, filtered $\left(\mathrm{mg} / \mathrm{L}\right.$ as $\left.\mathrm{CaCO}_{3}\right)$ & 90 & 300 & 74 & 182 & 185 \\
\hline Calcium, filtered (mg/L) & 90 & 83 & 21 & 52 & 54 \\
\hline Magnesium, filtered $(\mathrm{mg} / \mathrm{L})$ & 90 & 22 & 5.2 & 12 & 13 \\
\hline Arsenic, unfiltered recoverable $(\mu \mathrm{g} / \mathrm{L})$ & 90 & 62 & 8 & 17 & 14 \\
\hline Arsenic, filtered $(\mu \mathrm{g} / \mathrm{L})$ & 90 & 20 & 6.6 & 11 & 10 \\
\hline Cadmium, unfiltered recoverable $(\mu \mathrm{g} / \mathrm{L})$ & 90 & 2 & $<.1$ & ${ }^{2} .2$ & $<1$ \\
\hline Cadmium, filtered $(\mu \mathrm{g} / \mathrm{L})$ & 90 & .2 & $<.1$ & ${ }^{2}<.1$ & $<.1$ \\
\hline Copper, unfiltered recoverable $(\mu \mathrm{g} / \mathrm{L})$ & 88 & 360 & 4.6 & 50 & 26 \\
\hline Copper, filtered $(\mu \mathrm{g} / \mathrm{L})$ & 88 & 21 & 1 & 7 & 6 \\
\hline Iron, unfiltered recoverable $(\mu \mathrm{g} / \mathrm{L})$ & 89 & 8,800 & 20 & 1,150 & 560 \\
\hline Iron, filtered $(\mu \mathrm{g} / \mathrm{L})$ & 90 & 150 & $<3$ & ${ }^{2} 20$ & 9 \\
\hline Lead, unfiltered recoverable $(\mu \mathrm{g} / \mathrm{L})$ & 86 & 56 & $<1$ & ${ }^{2} 9$ & 4 \\
\hline Lead, filtered $(\mu \mathrm{g} / \mathrm{L})$ & 86 & 1.2 & $<.08$ & ${ }^{2} .2$ & $<.5$ \\
\hline Manganese, unfiltered recoverable $(\mu \mathrm{g} / \mathrm{L})$ & 90 & 880 & 8 & 164 & 105 \\
\hline Manganese, filtered $(\mu \mathrm{g} / \mathrm{L})$ & 90 & 60.7 & 4.5 & 18 & 15 \\
\hline Zinc, unfiltered recoverable $(\mu \mathrm{g} / \mathrm{L})$ & 90 & 490 & 3 & 73 & 40 \\
\hline Zinc, filtered $(\mu \mathrm{g} / \mathrm{L})$ & 90 & 21 & $<3$ & 27 & 5 \\
\hline Sediment, suspended concentration $(\mathrm{mg} / \mathrm{L})$ & 91 & 530 & 2 & 74 & 30 \\
\hline Sediment, suspended discharge (ton/d) & 91 & 4,720 & 1.7 & 380 & 66 \\
\hline Sediment, suspended (percent finer than $0.062 \mathrm{~mm}$ ) & 91 & 92 & 38 & 73 & 74 \\
\hline
\end{tabular}


TABLE 21

Table 21. Statistical summary of water-quality data for the upper Clark Fork basin, Montana, March 1985 through September 2003-Continued

\begin{tabular}{|c|c|c|c|c|c|}
\hline Property or constituent and reporting unit & $\begin{array}{l}\text { Number of } \\
\text { samples }\end{array}$ & Maximum & Minimum & Mean & Median \\
\hline \multicolumn{6}{|c|}{$\begin{array}{l}\text { 12334510--ROCK CREEK NEAR CLINTON, MONT. } \\
\text { Period of record for water-quality data: March 1985-September } 2003\end{array}$} \\
\hline Streamflow, instantaneous $\left(\mathrm{ft}^{3} / \mathrm{s}\right)$ & 103 & 5,060 & 113 & 970 & 548 \\
\hline Specific conductance, onsite $(\mu \mathrm{S} / \mathrm{cm})$ & 94 & 160 & 53 & 107 & 102 \\
\hline Temperature, water $\left({ }^{\circ} \mathrm{C}\right)$ & 103 & 18 & 0.0 & 8.3 & 8.5 \\
\hline $\mathrm{pH}$, onsite (standard units) & 93 & 8.8 & 6.9 & 8.0 & 8.0 \\
\hline Hardness, filtered $\left(\mathrm{mg} / \mathrm{L}\right.$ as $\left.\mathrm{CaCO}_{3}\right)$ & 85 & 90 & 22 & 50 & 50 \\
\hline Calcium, filtered (mg/L) & 85 & 23.0 & 5.92 & 13 & 13 \\
\hline Magnesium, filtered (mg/L) & 85 & 8.00 & 1.86 & 4.3 & 4.3 \\
\hline Arsenic, unfiltered recoverable $(\mu \mathrm{g} / \mathrm{L})$ & 91 & 5 & $<1$ & 2.9 & $<1$ \\
\hline Arsenic, filtered $(\mu \mathrm{g} / \mathrm{L})$ & 91 & 1 & $<1$ & 2.6 & $<1$ \\
\hline Cadmium, unfiltered recoverable $(\mu \mathrm{g} / \mathrm{L})$ & 91 & 3 & $<.04$ & 2.1 & $<1$ \\
\hline Cadmium, filtered $(\mu \mathrm{g} / \mathrm{L})$ & 91 & 1 & $<.04$ & -- & $<.1$ \\
\hline Copper, unfiltered recoverable $(\mu \mathrm{g} / \mathrm{L})$ & 89 & 41 & $<.6$ & ${ }^{2} 4$ & 2 \\
\hline Copper, filtered $(\mu \mathrm{g} / \mathrm{L})$ & 90 & 6 & $<1$ & ${ }^{2} 1$ & $<1$ \\
\hline Iron, unfiltered recoverable $(\mu \mathrm{g} / \mathrm{L})$ & 91 & 2,100 & 20 & 298 & 130 \\
\hline Iron, filtered $(\mu \mathrm{g} / \mathrm{L})$ & 91 & 163 & 5 & ${ }^{2} 34$ & 28 \\
\hline Lead, unfiltered recoverable $(\mu \mathrm{g} / \mathrm{L})$ & 89 & 19 & $<.06$ & $2_{2}$ & $<1$ \\
\hline Lead, filtered $(\mu \mathrm{g} / \mathrm{L})$ & 89 & 5 & $<.08$ & ${ }^{2} .4$ & $<1$ \\
\hline Manganese, unfiltered recoverable $(\mu \mathrm{g} / \mathrm{L})$ & 91 & 90 & $<10$ & ${ }^{2} 15$ & 7 \\
\hline Manganese, filtered $(\mu \mathrm{g} / \mathrm{L})$ & 91 & 8 & $<1$ & ${ }^{2} 2$ & 2 \\
\hline Zinc, unfiltered recoverable $(\mu \mathrm{g} / \mathrm{L})$ & 91 & 60 & $<1$ & ${ }^{2} 6$ & $<10$ \\
\hline Zinc, filtered $(\mu \mathrm{g} / \mathrm{L})$ & 91 & 15 & $<1$ & ${ }^{2} 2$ & $<3$ \\
\hline Sediment, suspended concentration $(\mathrm{mg} / \mathrm{L})$ & 103 & 223 & 1 & 21 & 5 \\
\hline Sediment, suspended discharge (ton/d) & 103 & 3,050 & .31 & 142 & 9.8 \\
\hline Sediment, suspended (percent finer than $0.062 \mathrm{~mm}$ ) & 103 & 95 & 35 & 70 & 71 \\
\hline
\end{tabular}


78 Water-Quality, Bed-Sediment, and Biological Data (October 2002 through September 2003) and Statistical Summaries of Data for Streams in the Upper Clark Fork Basin, Montana

Table 21. Statistical summary of water-quality data for the upper Clark Fork basin, Montana, March 1985 through September 2003-Continued

\begin{tabular}{|c|c|c|c|c|c|}
\hline Property or constituent and reporting unit & $\begin{array}{c}\text { Number of } \\
\text { samples }\end{array}$ & Maximum & Minimum & Mean & Median \\
\hline \multicolumn{6}{|c|}{ 12334550-CLARK FORK AT TURAH BRIDGE, NEAR BONNER, MONT. } \\
\hline Streamflow, instantaneous $\left(\mathrm{ft}^{3} / \mathrm{s}\right)$ & 188 & 9,560 & 296 & 1,890 & 1,140 \\
\hline Specific conductance, onsite $(\mu \mathrm{S} / \mathrm{cm})$ & 163 & 483 & 139 & 304 & 318 \\
\hline Temperature, water $\left({ }^{\circ} \mathrm{C}\right)$ & 187 & 22.0 & 0.0 & 9.3 & 9.5 \\
\hline $\mathrm{pH}$, onsite (standard units) & 134 & 8.8 & 7.4 & 8.2 & 8.3 \\
\hline Hardness, filtered $\left(\mathrm{mg} / \mathrm{L}\right.$ as $\left.\mathrm{CaCO}_{3}\right)$ & 124 & 210 & 54 & 132 & 130 \\
\hline Calcium, filtered $(\mathrm{mg} / \mathrm{L})$ & 124 & 59.0 & 14.9 & 37 & 38 \\
\hline Magnesium, filtered (mg/L) & 124 & 14.0 & 3.94 & 9.4 & 9.4 \\
\hline Arsenic, unfiltered recoverable $(\mu \mathrm{g} / \mathrm{L})$ & 133 & 110 & 3 & 11 & 7 \\
\hline Arsenic, filtered $(\mu \mathrm{g} / \mathrm{L})$ & 133 & 17 & 2.7 & 6 & 5 \\
\hline Cadmium, unfiltered recoverable $(\mu \mathrm{g} / \mathrm{L})$ & 133 & 4 & $<.1$ & 2.3 & $<1$ \\
\hline Cadmium, filtered $(\mu g / L)$ & 133 & .11 & $<.04$ & ${ }^{2} .04$ & $<.1$ \\
\hline Copper, unfiltered recoverable $(\mu \mathrm{g} / \mathrm{L})$ & 131 & 500 & 3 & 41 & 19 \\
\hline Copper, filtered $(\mu \mathrm{g} / \mathrm{L})$ & 132 & 25 & E1.1 & 5 & 4 \\
\hline Iron, unfiltered recoverable $(\mu \mathrm{g} / \mathrm{L})$ & 133 & 19,000 & 40 & 1,210 & 480 \\
\hline Iron, filtered $(\mu \mathrm{g} / \mathrm{L})$ & 133 & 190 & $<3$ & ${ }^{2} 26$ & 15 \\
\hline Lead, unfiltered recoverable $(\mu \mathrm{g} / \mathrm{L})$ & 129 & 100 & $<1$ & 29 & 4 \\
\hline Lead, filtered $(\mu \mathrm{g} / \mathrm{L})$ & 129 & 7 & $<.08$ & ${ }^{2} .4$ & $<1$ \\
\hline Manganese, unfiltered recoverable $(\mu \mathrm{g} / \mathrm{L})$ & 133 & 2,000 & 10 & 143 & 71 \\
\hline Manganese, filtered $(\mu \mathrm{g} / \mathrm{L})$ & 133 & 37.4 & 1.0 & 8 & 7 \\
\hline Zinc, unfiltered recoverable $(\mu \mathrm{g} / \mathrm{L})$ & 133 & 1,100 & $<10$ & ${ }^{2} 71$ & 36 \\
\hline Zinc, filtered $(\mu \mathrm{g} / \mathrm{L})$ & 132 & 39 & $<3$ & 27 & 5 \\
\hline Sediment, suspended concentration $(\mathrm{mg} / \mathrm{L})$ & 188 & 1,370 & 2 & 63 & 22 \\
\hline Sediment, suspended discharge (ton/d) & 188 & 34,700 & 3.5 & 704 & 64 \\
\hline Sediment, suspended (percent finer than $0.062 \mathrm{~mm}$ ) & 177 & 98 & 27 & 73 & 74 \\
\hline
\end{tabular}


Table 21. Statistical summary of water-quality data for the upper Clark Fork basin, Montana, March 1985 through September 2003-Continued

\begin{tabular}{|c|c|c|c|c|c|}
\hline Property or constituent and reporting unit & $\begin{array}{l}\text { Number of } \\
\text { samples }\end{array}$ & Maximum & Minimum & Mean & Median \\
\hline \multicolumn{6}{|c|}{ 12340000--BLACKFOOT RIVER NEAR BONNER, MONT. } \\
\hline \multicolumn{6}{|c|}{ Period of record for water-quality data: March 1985-September 2003} \\
\hline Streamflow, instantaneous $\left(\mathrm{ft}^{3} / \mathrm{s}\right)$ & 134 & 13,400 & 344 & 2,720 & 1,310 \\
\hline Specific conductance, onsite $(\mu \mathrm{S} / \mathrm{cm})$ & 111 & 294 & 131 & 207 & 205 \\
\hline Temperature, water $\left({ }^{\circ} \mathrm{C}\right)$ & 134 & 21.0 & 0.0 & 9.2 & 9.5 \\
\hline $\mathrm{pH}$, onsite (standard units) & 94 & 8.7 & 7.5 & 8.3 & 8.3 \\
\hline Hardness, filtered $\left(\mathrm{mg} / \mathrm{L}\right.$ as $\left.\mathrm{CaCO}_{3}\right)$ & 87 & 140 & 55 & 102 & 97 \\
\hline Calcium, filtered (mg/L) & 87 & 37 & 14 & 26 & 25 \\
\hline Magnesium, filtered (mg/L) & 87 & 13 & 4.9 & 9.0 & 8.6 \\
\hline Arsenic, unfiltered recoverable $(\mu \mathrm{g} / \mathrm{L})$ & 94 & 4 & $<1$ & ${ }^{2} 1$ & 1 \\
\hline Arsenic, filtered $(\mu \mathrm{g} / \mathrm{L})$ & 94 & 2 & $<1$ & ${ }^{2} .9$ & 1 \\
\hline Cadmium, unfiltered recoverable $(\mu \mathrm{g} / \mathrm{L})$ & 94 & 2 & $<.04$ & ${ }^{2} .2$ & $<1$ \\
\hline Cadmium, filtered $(\mu \mathrm{g} / \mathrm{L})$ & 94 & 1 & $<.04$ & -- & $<.1$ \\
\hline Copper, unfiltered recoverable $(\mu \mathrm{g} / \mathrm{L})$ & 91 & 34 & $<1$ & ${ }^{2} 6$ & 3 \\
\hline Copper, filtered $(\mu \mathrm{g} / \mathrm{L})$ & 92 & 7 & $<1$ & ${ }^{2} 2$ & 1 \\
\hline Iron, unfiltered recoverable $(\mu \mathrm{g} / \mathrm{L})$ & 94 & 3,600 & 10 & 503 & 230 \\
\hline Iron, filtered $(\mu \mathrm{g} / \mathrm{L})$ & 94 & 100 & $<3$ & ${ }^{2} 19$ & 12 \\
\hline Lead, unfiltered recoverable $(\mu \mathrm{g} / \mathrm{L})$ & 90 & 25 & $<.06$ & 23 & $<1$ \\
\hline Lead, filtered $(\mu \mathrm{g} / \mathrm{L})$ & 90 & 8 & $<.08$ & 2.5 & $<1$ \\
\hline Manganese, unfiltered recoverable $(\mu \mathrm{g} / \mathrm{L})$ & 94 & 180 & $<10$ & ${ }^{2} 33$ & 20 \\
\hline Manganese, filtered $(\mu \mathrm{g} / \mathrm{L})$ & 94 & 11 & $<1$ & ${ }^{2} 3$ & 2 \\
\hline Zinc, unfiltered recoverable $(\mu \mathrm{g} / \mathrm{L})$ & 94 & 60 & $<1$ & ${ }^{2} 7$ & $<10$ \\
\hline Zinc, filtered $(\mu \mathrm{g} / \mathrm{L})$ & 94 & 15 & $<1$ & ${ }^{2} 3$ & $<3$ \\
\hline Sediment, suspended concentration $(\mathrm{mg} / \mathrm{L})$ & 134 & 271 & 1 & 32 & 9 \\
\hline Sediment, suspended discharge (ton/d) & 134 & 7,670 & 1.1 & 575 & 31 \\
\hline Sediment, suspended (percent finer than $0.062 \mathrm{~mm}$ ) & 132 & 98 & 42 & 80 & 82 \\
\hline
\end{tabular}



in the Upper Clark Fork Basin, Montana

Table 21. Statistical summary of water-quality data for the upper Clark Fork basin, Montana, March 1985 through September 2003-Continued

\begin{tabular}{|c|c|c|c|c|c|}
\hline Property or constituent and reporting unit & $\begin{array}{c}\text { Number of } \\
\text { samples }\end{array}$ & Maximum & Minimum & Mean & Median \\
\hline \multicolumn{6}{|c|}{ 12340500-CLARK FORK ABOVE MISSOULA, MONT. } \\
\hline \multicolumn{6}{|c|}{ Period of record for water-quality data: July 1986-September 2003} \\
\hline Streamflow, instantaneous $\left(\mathrm{ft}^{3} / \mathrm{s}\right)$ & 154 & 21,600 & 720 & 4,510 & 2,400 \\
\hline Specific conductance, onsite $(\mu \mathrm{S} / \mathrm{cm})$ & 131 & 399 & 142 & 253 & 261 \\
\hline Temperature, water $\left({ }^{\circ} \mathrm{C}\right)$ & 151 & 21.0 & 0.0 & 9.3 & 8.5 \\
\hline $\mathrm{pH}$, onsite (standard units) & 111 & 8.7 & 7.9 & 8.3 & 8.3 \\
\hline Hardness, filtered (mg/L as $\left.\mathrm{CaCO}_{3}\right)$ & 111 & 170 & 61 & 116 & 120 \\
\hline Calcium, filtered (mg/L) & 111 & 46 & 14 & 31 & 32 \\
\hline Magnesium, filtered (mg/L) & 111 & 13.0 & 5.28 & 9.2 & 9.2 \\
\hline Arsenic, unfiltered recoverable $(\mu \mathrm{g} / \mathrm{L})$ & 111 & 69 & 1 & 5 & 4 \\
\hline Arsenic, filtered $(\mu \mathrm{g} / \mathrm{L})$ & 111 & 9 & 1 & 3 & 3 \\
\hline Cadmium, unfiltered recoverable $(\mu \mathrm{g} / \mathrm{L})$ & 111 & 5 & $<.1$ & 2.2 & $<1$ \\
\hline Cadmium, filtered $(\mu \mathrm{g} / \mathrm{L})$ & 111 & .2 & $<.04$ & ${ }^{2} .03$ & $<.1$ \\
\hline Copper, unfiltered recoverable $(\mu \mathrm{g} / \mathrm{L})$ & 109 & 400 & 2 & 17 & 8 \\
\hline Copper, filtered $(\mu \mathrm{g} / \mathrm{L})$ & 110 & 12.6 & .7 & 3 & 2 \\
\hline Iron, unfiltered recoverable $(\mu \mathrm{g} / \mathrm{L})$ & 111 & 13,000 & 40 & 643 & 250 \\
\hline Iron, filtered $(\mu \mathrm{g} / \mathrm{L})$ & 111 & 200 & $<3$ & ${ }^{2} 23$ & 16 \\
\hline Lead, unfiltered recoverable ( $\mu \mathrm{g} / \mathrm{L})$ & 106 & 78 & $<1$ & $2_{3}$ & 2 \\
\hline Lead, filtered $(\mu \mathrm{g} / \mathrm{L})$ & 106 & 1.2 & $<.08$ & 2.2 & $<.6$ \\
\hline Manganese, unfiltered recoverable $(\mu \mathrm{g} / \mathrm{L})$ & 111 & 1,100 & 10 & 68 & 40 \\
\hline Manganese, filtered $(\mu \mathrm{g} / \mathrm{L})$ & 111 & 230 & 6.2 & 18 & 14 \\
\hline Zinc, unfiltered recoverable $(\mu \mathrm{g} / \mathrm{L})$ & 111 & 1,100 & $<10$ & 233 & 13 \\
\hline Zinc, filtered $(\mu \mathrm{g} / \mathrm{L})$ & 111 & 16 & $<1$ & 24 & 2 \\
\hline Sediment, suspended concentration $(\mathrm{mg} / \mathrm{L})$ & 154 & 824 & 2 & 39 & 12 \\
\hline Sediment, suspended discharge (ton/d) & 154 & 21,900 & 5.8 & 1,010 & 81 \\
\hline Sediment, suspended (percent finer than $0.062 \mathrm{~mm}$ ) & 149 & 99 & 44 & 87 & 90 \\
\hline
\end{tabular}

${ }^{1}$ Differing less-than $(<)$ values for an individual constituent are the result of changes in the analytical detection limit during the period of record.

${ }^{2}$ Value is estimated by using a log-probability regression to predict the values of data less than the laboratory reporting level (Helsel and Cohn, 1988). 
Table 22. Statistical summary of fine-grained bed-sediment data for the upper Clark Fork basin, Montana, August 1986 through September 2003

[Fine-grained bed sediment is material less than 0.064 millimeter in diameter. Reported concentrations are in micrograms per gram dry weight. Number of samples represents the number of years that the constituent was analyzed, with each year represented by a single mean concentration of composite samples. Symbols: <, less than the analytical detection limit; --, indicates insufficient data to compute statistic]

\begin{tabular}{llllll}
\hline Constituent & $\begin{array}{c}\text { Number } \\
\text { of } \\
\text { samples }\end{array}$ & Maximum & Minimum & Mean & Median \\
\hline
\end{tabular}

2323600--SILVER BOW CREEK AT OPPORTUNITY, MONT.

Period of record for fine-grained bed-sediment data: 1992-2003

Arsenic

Cadmium

Chromium

Copper

Iron

Lead

Manganese

Nickel

Silver

Zinc

$\begin{array}{cc}42.0 & -- \\ 32.4 & 23.7 \\ 9,020 & 36.8 \\ 41,200 & 28,200 \\ 1,030 & 381 \\ 9,220 & 1,690 \\ 21.4 & 12.7 \\ 20.0 & 8.3 \\ 13,400 & 5,620\end{array}$

\section{6}

$\begin{array}{cc}33.1 & 32.4 \\ 25.7 & 26.2 \\ 5,090 & 4,740 \\ 36,300 & 37,500 \\ 762 & 815 \\ 3,430 & 2,680 \\ 15.7 & 15.5 \\ 15.5 & 15.8 \\ 8,460 & 7,820\end{array}$

\section{0--SILVER BOW CREEK AT WARM SPRINGS, MONT.}

Arsenic

Cadmium

Chromium

Copper

Iron

Lead

Manganese

Nickel

Silver

Zinc

Period of record for fine-grained bed-sediment data: 1992-2003

$\begin{array}{ccccc}1 & - & - & 103 & -- \\ 12 & 12.2 & 4.2 & 7.5 & 7.1 \\ 10 & 34.1 & 12.3 & 20.8 & 21.2 \\ 12 & 769 & 169 & 366 & 279 \\ 12 & 27,200 & 15,400 & 20,900 & 20,600 \\ 12 & 100 & 50 & 72 & 70 \\ 12 & 17,700 & 1,470 & 7,890 & 7,690 \\ 11 & 19.1 & 9.2 & 14.6 & 14.6 \\ 12 & 4.4 & .3 & 11.9 & 1.8 \\ 12 & 2,220 & 620 & 1,060 & 830\end{array}$

12323770--WARM SPRINGS CREEK AT WARM SPRINGS, MONT.

Period of record for fine-grained bed-sediment data: 1995, 1997, 1999, 2002

Cadmium
Chromium
Copper
Iron
Lead
Manganese
Nickel
Silver
Zinc

1.3
27.5
779
16,800
67
2,020
17.6
3.1
372

$\begin{array}{cc}3.4 & 3.2 \\ 30.8 & 31.1 \\ 850 & 864 \\ 20,400 & 21,200 \\ 81 & 85 \\ 6,950 & 7,410 \\ 19.2 & 18.6 \\ 3.8 & 3.5 \\ 391 & 385\end{array}$

12323800--CLARK FORK NEAR GALEN, MONT.

Period of record for fine-grained bed-sediment data: 1987, 1991-2003

Arsenic
Cadmium
Chromium
Copper
Iron
Lead
Manganese
Nickel
Silver
Zinc

$\begin{array}{cc}-- & -- \\ 20.1 & 4.0 \\ 33.9 & 19.1 \\ 2,300 & 991 \\ 39,800 & 22,600 \\ 235 & 113 \\ 15,600 & 2,780 \\ 23.2 & 15.4 \\ 7.3 & <3.2 \\ 3,560 & 1,090\end{array}$

1,090

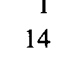

10

14

14

14

14

11

14

$\begin{array}{cc}1 & -- \\ 4 & 20.1 \\ 0 & 33.9 \\ 4 & 2,300 \\ 4 & 39,800 \\ 4 & 235 \\ 4 & 15,600 \\ 1 & 23.2 \\ 4 & 7.3 \\ 4 & 3,560\end{array}$

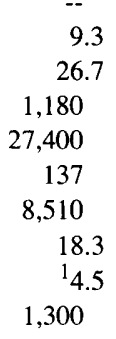

$\begin{array}{cc}111 & -- \\ 10.0 & 9.3 \\ 27.0 & 26.7 \\ 1,280 & 1,180 \\ 28,200 & 27,400 \\ 145 & 137 \\ 8,840 & 8,510 \\ 18.7 & 18.3 \\ 14.4 & 14.5 \\ 1,690 & 1,300\end{array}$


82 Water-Quality, Bed-Sediment, and Biological Data (October 2002 through September 2003) and Statistical Summaries of Data for Streams in the Upper Clark Fork Basin, Montana

Table 22. Statistical summary of fine-grained bed-sediment data for the upper Clark Fork basin, Montana, August 1986 through September 2003-Continued

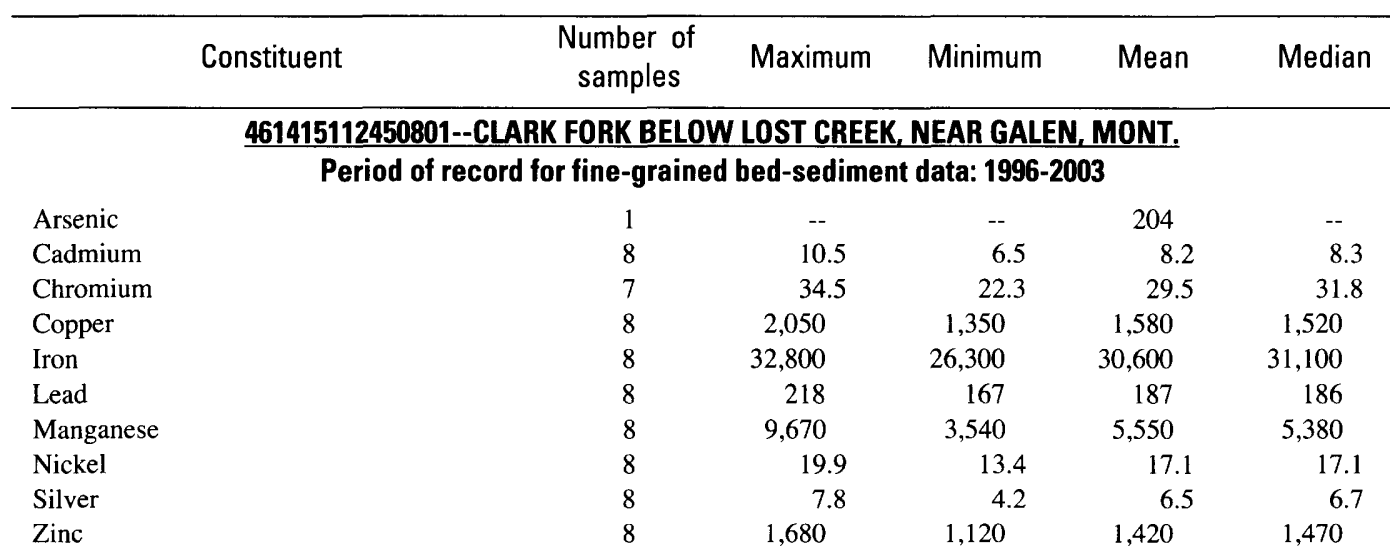

461559112443301--CLARK FORK NEAR RACETRACK, MONT.

Period of record for fine-grained bed-sediment data: 1996-2003

$\begin{array}{llcccc}\text { Arsenic } & 1 & - & -- & 101 & -- \\ \text { Cadmium } & 8 & 8.7 & 5.0 & 7.3 & 7.8 \\ \text { Chromium } & 7 & 33.3 & 19.1 & 26.7 & 29.2 \\ \text { Copper } & 8 & 1,610 & 933 & 1,240 & 1,280 \\ \text { Iron } & 8 & 31,700 & 23,100 & 27,300 & 28,400 \\ \text { Lead } & 8 & 186 & 128 & 151 & 148 \\ \text { Manganese } & 8 & 6,310 & 2,100 & 3,460 & 3,020 \\ \text { Nickel } & 8 & 18.4 & 10.5 & 14.7 & 16.0 \\ \text { Silver } & 8 & 6.1 & <3.3 & 15.0 & 15.4 \\ \text { Zinc } & 8 & 1,550 & 1,030 & 1,250 & 1,180\end{array}$

461903112440701--CLARK FORK AT DEMPSEY CREEK DIVERSION, NEAR RACETRACK, MONT.

Period of record for fine-grained bed-sediment data: 1996-2003

Arsenic

Cadmium

Chromium

Copper

Iron

Lead

Manganese

Nickel

Silver

Zinc

-
10.3
34.1
1,550
33,700
155
8,370
16.9
6.2
1,570

$\begin{array}{cc}80 & -- \\ 7.2 & 7.5 \\ 26.3 & 26.4 \\ 1,070 & 1,060 \\ 27,100 & 26,800 \\ 135 & 134 \\ 3,870 & 2,660 \\ 13.5 & 14.0 \\ 4.9 & 5.0 \\ 1,160 & 1,150\end{array}$

12324200--CLARK FORK AT DEER LODGE, MONT.

Arsenic
Cadmium
Chromium
Copper
Iron
Lead
Manganese
Nickel
Silver
Zinc

\section{Period of record for fine-grained bed-sediment data: 1986-87, 1990-2003}

$\begin{array}{cccc}-- & - & 77 & - \\ 10.0 & 4.4 & 6.9 & 6.8 \\ 43.9 & 19.5 & 32.0 & 33.9 \\ 4,180 & 837 & 1,380 & 1,070 \\ 35,300 & 22,600 & 27,900 & 27,600 \\ 242 & 121 & 155 & 150 \\ 6,020 & 1,460 & 2,850 & 2,420 \\ 21.1 & 12.2 & 16.1 & 15.3 \\ 7.9 & 2.4 & 4.7 & 4.5 \\ 1,730 & 940 & 1,290 & 1,290\end{array}$


Table 22. Statistical summary of fine-grained bed-sediment data for the upper Clark Fork basin, Montana, August 1986 through September 2003-Continued

\begin{tabular}{|c|c|c|c|c|c|}
\hline & $\begin{array}{c}\text { Number of } \\
\text { samples }\end{array}$ & Maximum & Minimum & Mean & Median \\
\hline \multicolumn{6}{|c|}{ 12324590--LITTLE BLACKFOOT RIVER NEAR GARRISON, MONT. } \\
\hline \multicolumn{6}{|c|}{ Period of record for fine-grained bed-sediment data: 1986-87, 1994, 1998, 2001} \\
\hline Cadmium & 5 & 2.3 & .2 & 1.1 & .9 \\
\hline Chromium & 3 & 54.4 & 22.1 & 43.1 & 52.9 \\
\hline Copper & 5 & 85 & 38 & 56 & 40 \\
\hline Iron & 5 & 30,700 & 16,100 & 24,200 & 24,200 \\
\hline Lead & 5 & 53 & 36 & 40 & 37 \\
\hline Manganese & 5 & 2,700 & 905 & 1,420 & 1,040 \\
\hline Nickel & 3 & 21.9 & 13.6 & 17.7 & 17.6 \\
\hline Silver & 5 & .9 & $<.5$ & ${ }^{1} .6$ & ${ }^{1} .8$ \\
\hline Zinc & 5 & 204 & 161 & 176 & 170 \\
\hline
\end{tabular}

12324680--CLARK FORK AT GOLDCREEK, MONT.

Period of record for fine-grained bed-sediment data: 1992-2003

$\begin{array}{lccccc}\text { Arsenic } & 1 & -- & - & 32 & -- \\ \text { Cadmium } & 12 & 8.1 & 3.5 & 5.4 & 5.7 \\ \text { Chromium } & 10 & 48.9 & 24.9 & 33.7 & 32.6 \\ \text { Copper } & 12 & 1,080 & 393 & 726 & 757 \\ \text { Iron } & 12 & 30,600 & 19,500 & 24,200 & 24,400 \\ \text { Lead } & 12 & 152 & 61 & 101 & 106 \\ \text { Manganese } & 12 & 2,610 & 1,160 & 1,840 & 1,810 \\ \text { Nickel } & 11 & 18.6 & 10.9 & 15.2 & 15.9 \\ \text { Silver } & 12 & 4.8 & 2.3 & 3.3 & 3.2 \\ \text { Zinc } & 12 & 1,320 & 590 & 1,020 & 1,100\end{array}$

12331500--FLINT CREEK NEAR DRUMMOND, MONT.

Period of record for fine-grained bed-sediment data: 1986, 1989, 1992-2003

Arsenic

Chromium

Copper

Iron

Lead

Manganese

Nickel

Silver

Zinc
Cadmium

1
14
10
14
14
14
14
11
13
14

--
$<.2$
20.4
47
19,800
126
2,370
10.4
5.0
503

$\begin{array}{cc}113 & -- \\ 1_{3.5} & 1_{3.2} \\ 25.0 & 25.2 \\ 60 & 61 \\ 23,400 & 23,300 \\ 173 & 171 \\ 3,930 & 4,040 \\ 12.4 & 11.7 \\ 6.4 & 6.4 \\ 635 & 646\end{array}$

12331800--CLARK FORK NEAR DRUMMOND, MONT.

Period of record for fine-grained bed-sediment data: 1986-87, 1991-2003

Arsenic
Cadmium
Chromium
Copper
Iron
Lead
Manganese
Nickel
Silver
Zinc

Arsenic

Chromium

Copper

Lead

Zinc

$\begin{array}{cc}-- & -- \\ 7.7 & 2.6 \\ 35.4 & 17.0 \\ 747 & 321 \\ 27,000 & 16,500 \\ 135 & 68 \\ 3,090 & 1,150 \\ 16.8 & 10.8 \\ 4.7 & <3.2 \\ 1,230 & 761\end{array}$

$\begin{array}{cc}33 & -- \\ 4.9 & 4.8 \\ 30.1 & 32.1 \\ 512 & 499 \\ 21,800 & 23,200 \\ 94 & 94 \\ 1,860 & 1,740 \\ 14.2 & 15.0 \\ 13.0 & 1^{2.9} \\ 1,020 & 1,030\end{array}$


84 Water-Quality, Bed-Sediment, and Biological Data (October 2002 through September 2003) and Statistical Summaries of Data for Streams in the Upper Clark Fork Basin, Montana

Table 22. Statistical summary of fine-grained bed-sediment data for the upper Clark Fork basin, Montana, August 1986 through September 2003-Continued

\begin{tabular}{|c|c|c|c|c|c|c|}
\hline & Constituent & $\begin{array}{c}\text { Number of } \\
\text { samples }\end{array}$ & Maximum & Minimum & Mean & Median \\
\hline \multicolumn{7}{|c|}{ 12334510--ROCK CREEK NEAR CLINTON, MONT. } \\
\hline \multicolumn{7}{|c|}{ Period of record for fine-grained bed-sediment data: 1986-87, 1989, 1991-99, 2001-03 } \\
\hline Arsenic & & 1 & -- & -. & 5 & -- \\
\hline Cadmium & & 15 & 3.7 & $<.2$ & ${ }^{1} .9$ & ${ }^{1}<.8$ \\
\hline Chromium & & 9 & 27.9 & 16.5 & 22.2 & 21.3 \\
\hline Copper & & 15 & 16 & 3 & 12 & 13 \\
\hline Iron & & 15 & 21,400 & 13,100 & 17,600 & 17,600 \\
\hline Lead & & 15 & 16 & $<3$ & ${ }^{1} 9$ & ${ }^{1} 10$ \\
\hline Manganese & & 15 & 724 & 126 & 382 & 382 \\
\hline Nickel & & 10 & 14.8 & 9.5 & 12.1 & 12.1 \\
\hline Silver & & 14 & 1.9 & $<.3$ & ${ }^{1} .5$ & ${ }^{1}<.5$ \\
\hline Zinc & & 15 & 58 & 23 & 44 & 47 \\
\hline
\end{tabular}

12334550--CLARK FORK AT TURAH BRIDGE, NEAR BONNER, MONT.

Period of record for fine-grained bed-sediment data: 1986, 1991-2003

Arsenic

Cadmium

Chromium

Copper

Iron

Lead

Manganese

Nickel

Silver

Zinc

$\begin{array}{cccc}- & -- & 19 & -- \\ 7.3 & 3.1 & 4.2 & 3.9 \\ 34.7 & 15.3 & 26.0 & 28.4 \\ 635 & 211 & 376 & 338 \\ 24,400 & 12,600 & 19,000 & 17,400 \\ 115 & 47 & 73 & 68 \\ 2,270 & 671 & 1,200 & 1,110 \\ 19.1 & 8.7 & 13.3 & 12.0 \\ 3.9 & <1.9 & 12.1 & 11.9 \\ 1,160 & 586 & 874 & 861\end{array}$

12340000--BLACKFOOT RIVER NEAR BONNER, MONT.

Period of record for fine-grained bed-sediment data: 1986-87, 1991, 1993-96, 1998-2001, 2003

$\begin{array}{lccccc}\text { Arsenic } & 1 & -- & -- & 2 & -- \\ \text { Cadmium } & 12 & 2.0 & <.2 & 1.7 & 1<1.2 \\ \text { Chromium } & 8 & 25.8 & 15.1 & 20.5 & 21.8 \\ \text { Copper } & 12 & 27 & 11 & 20 & 21 \\ \text { Iron } & 12 & 20,200 & 12,400 & 16,800 & 16,800 \\ \text { Lead } & 12 & 20 & <13 & 1.2 & 112 \\ \text { Manganese } & 12 & 683 & 298 & 531 & 538 \\ \text { Nickel } & 9 & 14.3 & 9.4 & 11.9 & 12.5 \\ \text { Silver } & 12 & 1.0 & <.3 & 1.5 & 1.3 \\ \text { Zinc } & 12 & 73 & 35 & 58 & 61\end{array}$

12340500--CLARK FORK ABOVE MISSOULA, MONT.

Period of record for fine-grained bed-sediment data: 1997-2003

Arsenic
Cadmium
Chromium
Copper
Iron
Lead
Manganese
Nickel
Silver
Zinc

5.8
30.6
543
24,300
78
1,420
15.8
2.9
1,090

--
1.5
19.0
166
18,100
37
480
11.8
.8
438

23
3.5
26.6
326
20,600
55
1,040
13.5
12.0
703

29.3

282

20,400

54

1,160

13.3

${ }^{1} 2.1$

696 
Table 22. Statistical summary of fine-grained bed-sediment data for the upper Clark Fork basin, Montana, August 1986 through September 2003-Continued

\begin{tabular}{|c|c|c|c|c|c|c|}
\hline & Constituent & $\begin{array}{c}\text { Number of } \\
\text { samples }\end{array}$ & Maximum & Minimum & Mean & Median \\
\hline \multicolumn{7}{|c|}{ 12353000--CLARK FORK BELOW MISSOULA, MONT. ${ }^{2}$} \\
\hline \multicolumn{7}{|c|}{ Period of record for fine-grained bed-sediment data: 1986, 1990-2003 } \\
\hline Arsenic & & 1 & -- & -- & 6 & -- \\
\hline Cadmium & & 15 & 6.0 & 1.1 & 2.4 & 1.9 \\
\hline Chromium & & 10 & 27.6 & 12.3 & 21.9 & 22.6 \\
\hline Copper & & 15 & 293 & 69 & 148 & 138 \\
\hline Iron & & 15 & 21,100 & 13,100 & 18,000 & 18,600 \\
\hline Lead & & 15 & 58 & 12 & 36 & 36 \\
\hline Manganese & & 15 & 2,530 & 446 & 1,320 & 1,260 \\
\hline Nickel & & 11 & 14.1 & 8.4 & 12.0 & 12.5 \\
\hline Silver & & 15 & 3.0 & .4 & ${ }^{1} 1.3$ & ${ }^{1} 1.3$ \\
\hline Zinc & & 15 & 675 & 239 & 391 & 380 \\
\hline
\end{tabular}

${ }^{1}$ Value determined by arbitrarily substituting one-half of the analytical detection limit for censored $(<)$ values when both uncensored and censored values were used in determining the mean and (or) median. When all data were less than the analytical detection limit, the median was determined by ranking the censored values in order of magnitude.

${ }^{2}$ Samples collected about 30 miles downstream from streamflow-gaging station to conform to previous sampling location. 
86 Water-Quality, Bed-Sediment, and Biological Data (October 2002 through September 2003) and Statistical Summaries of Data for Streams in the Upper Clark Fork Basin, Montana

Table 23. Statistical summary of bulk bed-sediment data for the upper Clark Fork basin, Montana, August 1993 through September 2003

[Bulk bed sediment is material smaller than about 10 millimeters in diameter. Reported concentrations are in micrograms per gram dry weight. Number of samples represents the number of years that the constituent was analyzed, with each year represented by a single mean concentration of composite samples. Symbols: <, less than the analytical detection limit; ,-- indicates insufficient data to compute statistic]

\begin{tabular}{|c|c|c|c|c|c|c|}
\hline & Constituent & $\begin{array}{c}\text { Number of } \\
\text { samples }\end{array}$ & Maximum & Minimum & Mean & Median \\
\hline \multicolumn{7}{|c|}{ 12323600--SILVER BOW CREEK AT OPPORTUNITY, MONT. } \\
\hline \multicolumn{7}{|c|}{ Period of record for bulk bed-sediment data: 1993-95, 1997-2003 } \\
\hline Arsenic & & 1 & -- & -- & 148 & -- \\
\hline Cadmium & & 10 & 30.2 & 4.2 & 13.7 & 10.6 \\
\hline Chromium & & 9 & 18.1 & 9.6 & 13.5 & 12.7 \\
\hline Copper & & 10 & 3,800 & 670 & 1,580 & 1,260 \\
\hline Iron & & 10 & 29,300 & 18,300 & 23,500 & 23,300 \\
\hline Lead & & 10 & 398 & 198 & 289 & 270 \\
\hline Manganese & & 10 & 5,480 & 504 & 1,870 & 1,210 \\
\hline Nickel & & 10 & 12.7 & 6.0 & 8.1 & 7.7 \\
\hline Silver & & 10 & 8.0 & 3.2 & 5.1 & 4.4 \\
\hline Zinc & & 10 & 5,930 & 1,720 & 3,310 & 2,840 \\
\hline
\end{tabular}

12323750--SILVER BOW CREEK AT WARM SPRINGS, MONT.

Period of record for bulk bed-sediment data: 1993, 1995-2003

Arsenic

Cadmium

Chromium

Copper

Iron

Lead

Manganese

Nickel

Silver

Zinc

Cadmium

Chromium

Copper

Iron

Lead

Manganese

Nickel

Silver

Zinc

\begin{tabular}{lccccr}
\multicolumn{7}{c}{ Period of record for bulk bed-sediment data: $\mathbf{1 9 9 3 - 2 0 0 3}$} \\
Arsenic & 1 & -- & -- & 97 & - \\
Cadmium & 11 & 8.2 & $<.9$ & 14.0 & 14.0 \\
Chromium & 10 & 23.7 & 4.2 & 15.5 & 15.4 \\
Copper & 11 & 902 & 223 & 500 & 542 \\
Iron & 11 & 31,300 & 9,930 & 19,900 & 19,500 \\
Lead & 11 & 158 & 41 & 82 & 79 \\
Manganese & 11 & 9,490 & 900 & 3,450 & 1,900 \\
Nickel & 11 & 15.2 & 4.9 & 8.8 & 8.8 \\
Silver & 11 & 5.2 & .7 & 12.1 & 1.6 \\
Zinc & 11 & 1,280 & 417 & 706 & 653
\end{tabular}

--
$<.9$
5.2
9
6,100
$<10$
209
4.8
$<.3$
93

36
${ }^{1} 1.3$
8.7
48
9,700
${ }^{1} 15$
1,000
6.4
${ }^{1} .7$
160

12323770--WARM SPRINGS CREEK AT WARM SPRINGS, MONT.

Period of record for bulk bed-sediment data: 1995, 1997, 1999, 2002

$\begin{array}{rrrr}1.5 & <.8 & 1.6 & 1<.9 \\ 12.0 & 7.4 & 10.2 & 10.7 \\ 238 & 127 & 193 & 204 \\ 12,700 & 8,010 & 10,200 & 10,000 \\ 38 & 18 & 29 & 29 \\ 4,240 & 1,220 & 2,640 & 2,540 \\ 8.5 & 5.7 & 7.2 & 7.4 \\ 1.3 & <.8 & 1.9 & 1.0 \\ 275 & 146 & 183 & 155\end{array}$

12323800--CLARK FORK NEAR GALEN, MONT. Period of record for bulk bed-sediment data: 1993-2003 
Table 23. Statistical summary of bulk bed-sediment data for the upper Clark Fork basin, Montana, August 1993 through September 2003-Continued

\begin{tabular}{|c|c|c|c|c|c|c|}
\hline & Constituent & $\begin{array}{c}\text { Number of } \\
\text { samples }\end{array}$ & Maximum & Minimum & Mean & Median \\
\hline \multicolumn{7}{|c|}{ 461415112450801--CLARK FORK BELOW LOST CREEK, NEAR GALEN, MONT } \\
\hline \multicolumn{7}{|c|}{ Period of record for bulk bed-sediment data: $1996-2003$} \\
\hline Arsenic & & 1 & -- & -- & 82 & -- \\
\hline Cadmium & & 8 & 5.2 & $<.9$ & ${ }^{1} 2.7$ & ${ }^{1} 2.8$ \\
\hline Chromium & & 7 & 17.5 & 6.8 & 11.7 & 12.0 \\
\hline Copper & & 8 & 763 & 238 & 427 & 411 \\
\hline Iron & & 8 & 21,000 & 12,300 & 16,600 & 16,700 \\
\hline Lead & & 8 & 104 & 41 & 70 & 71 \\
\hline Manganese & & 8 & 3,720 & 1,260 & 1,750 & 1,420 \\
\hline Nickel & & 8 & 8.4 & 4.2 & 6.5 & 6.8 \\
\hline Silver & & 8 & 2.8 & .8 & ${ }^{1} 1.7$ & ${ }^{1} 1.6$ \\
\hline Zinc & & 8 & 787 & 339 & 527 & 539 \\
\hline
\end{tabular}

461559112443301--CLARK FORK NEAR RACETRACK, MONT.

Period of record for bulk bed-sediment data: 1996-2003

Arsenic

Cadmium

Chromium

Copper

Iron

Lead

Manganese

Nickel

Silver

Zinc

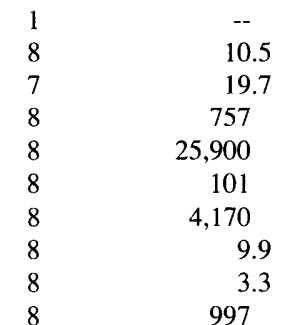

90
$1_{4.2}$
15.0
526
19,000
80
1,680
7.8
12.4
678

678

$$
\begin{gathered}
-- \\
13.0 \\
14.7 \\
535 \\
18,200 \\
81 \\
1,430 \\
8.2 \\
12.6
\end{gathered}
$$$$
679
$$

461903112440701--CLARK FORK AT DEMPSEY CREEK DIVERSION, NEAR RACETRACK, MONT.

Period of record for bulk bed-sediment data: 1996-2003

Arsenic
Cadmium
Chromium
Copper
Iron
Lead
Manganese
Nickel
Silver
Zinc

Arsenic

Cadmium

Chromium

Copper

Iron

Lead

Manganese

Nickel

Silver

Zinc

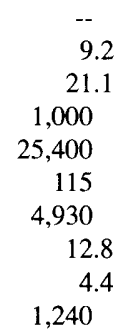
1.5
13.0
244
16,400
47
825
5.5
$<.8$

$\begin{array}{cc}82 & -- \\ 4.3 & 3.4 \\ 17.2 & 17.3 \\ 553 & 592 \\ 21,500 & 22,200 \\ 81 & 88 \\ 2,030 & 1,740 \\ 8.5 & 8.4 \\ 12.4 & 12.5 \\ 658 & 608\end{array}$

12324200--CLARK FORK AT DEER LODGE, MONT.

Period of record for bulk bed-sediment data: 1993-2003

$\begin{array}{rcccc}1 & -- & -- & 84 & -- \\ 11 & 8.3 & 1.0 & 1_{3.6} & 1_{2.4} \\ 10 & 29.2 & 12.1 & 19.1 & 19.6 \\ 11 & 906 & 281 & 525 & 440 \\ 11 & 25,000 & 13,200 & 19,800 & 20,200 \\ 11 & 122 & 45 & 81 & 82 \\ 11 & 3,560 & 607 & 1,430 & 1,050 \\ 11 & 12.3 & 7.7 & 10.2 & 10.2 \\ 11 & 3.9 & <.7 & 12.0 & { }^{1} 1.6 \\ 11 & 1,060 & 456 & 665 & 599\end{array}$


88 Water-Quality, Bed-Sediment, and Biological Data (October 2002 through September 2003) and Statistical Summaries of Data for Streams in the Upper Clark Fork Basin, Montana

Table 23. Statistical summary of bulk bed-sediment data for the upper Clark Fork basin, Montana,

August 1993 through September 2003-Continued

\begin{tabular}{|c|c|c|c|c|c|c|}
\hline & Constituent & $\begin{array}{c}\text { Number of } \\
\text { samples }\end{array}$ & Maximum & Minimum & Mean & Median \\
\hline \multicolumn{7}{|c|}{ 12324590--LITTLE BLACKFOOT RIVER NEAR GARRISON, MONT. } \\
\hline \multicolumn{7}{|c|}{ Period of record for bulk bed-sediment data: 1994, 1998, 2001} \\
\hline Cadmium & & 3 & $<1.5$ & $<1.2$ & ${ }^{1} .7$ & 1.7 \\
\hline Chromium & & 3 & 33.2 & 14.7 & 26.7 & 32.1 \\
\hline Copper & & 3 & 20 & 12 & 17 & 19 \\
\hline Iron & & 3 & 21,000 & 15,600 & 18,000 & 17,300 \\
\hline Lead & & 3 & 18 & 12 & 15 & 14 \\
\hline Manganese & & 3 & 420 & 308 & 354 & 333 \\
\hline Nickel & & 3 & 15.2 & 8.6 & 11.8 & 11.7 \\
\hline Silver & & 3 & $<1.6$ & $<.7$ & ${ }^{1} .6$ & ${ }^{1} .8$ \\
\hline Zinc & & 3 & 86 & 65 & 75 & 73 \\
\hline \multicolumn{7}{|c|}{ 12324680--CLARK FORK AT GOLDCREEK, MONT. } \\
\hline \multicolumn{7}{|c|}{ Period of record for bulk bed-sediment data: $1993-2003$} \\
\hline Arsenic & & 1 & -. & -- & 15 & -- \\
\hline Cadmium & & 11 & 7.6 & 1.1 & $1_{3.3}$ & $1_{3.2}$ \\
\hline Chromium & & 10 & 33.2 & 15.0 & 22.1 & 21.3 \\
\hline Copper & & 11 & 858 & 175 & 419 & 282 \\
\hline Iron & & 11 & 24,900 & 11,900 & 18,400 & 18,300 \\
\hline Lead & & 11 & 92 & 37 & 62 & 56 \\
\hline Manganese & & 11 & 2,930 & 377 & 1,230 & 930 \\
\hline Nickel & & 11 & 15.9 & 8.3 & 11.3 & 10.5 \\
\hline Silver & & 11 & 3.7 & $<.7$ & ${ }^{1} 1.8$ & ${ }^{1} 1.5$ \\
\hline Zinc & & 11 & 1,020 & 305 & 648 & 623 \\
\hline
\end{tabular}

12331500--FLINT CREEK NEAR DRUMMOND, MONT.

Period of record for bulk bed-sediment data: 1993-2003

Arsenic

Cadmium

Chromium

Copper

Iron

Lead

Manganese

Nickel

Silver

Zinc

$\begin{array}{rcc}1 & -- & - \\ 11 & 3.8 & <.2 \\ 10 & 13.9 & 4.9 \\ 11 & 40 & 16 \\ 11 & 15,700 & 8,630 \\ 11 & 120 & 51 \\ 11 & 3,200 & 1,150 \\ 11 & 8.0 & 4.5 \\ 11 & 5.8 & 2.5 \\ 11 & 429 & 178\end{array}$

$\begin{array}{cc}80 & -- \\ 1_{1.9} & { }_{1} 1.7 \\ 9.8 & 10.9 \\ 24 & 23 \\ 13,000 & 13,400 \\ 80 & 80 \\ 2,220 & 2,250 \\ 6.0 & 5.9 \\ 4.3 & 4.3 \\ 285 & 284\end{array}$

12331800--CLARK FORK NEAR DRUMMOND, MONT.

Period of record for bulk bed-sediment data: 1993-2003

Arsenic

Cadmium

Chromium

Copper

Iron

Lead

Manganese

Nickel

Silver

Zinc

$\begin{array}{cc}1 & -- \\ 11 & 5.1 \\ 10 & 29.5 \\ 11 & 605 \\ 11 & 21,800 \\ 11 & 78 \\ 11 & 3,280 \\ 11 & 14.2 \\ 11 & 3.5 \\ 11 & 939\end{array}$

--
$<1.6$
6.9
114
12,100
31
409
7.7
.5
381

31
$1_{2.7}$
18.4
265
16,000
49
1,190
10.3
1.7
586


Table 23. Statistical summary of bulk bed-sediment data for the upper Clark Fork basin, Montana,

August 1993 through September 2003-Continued

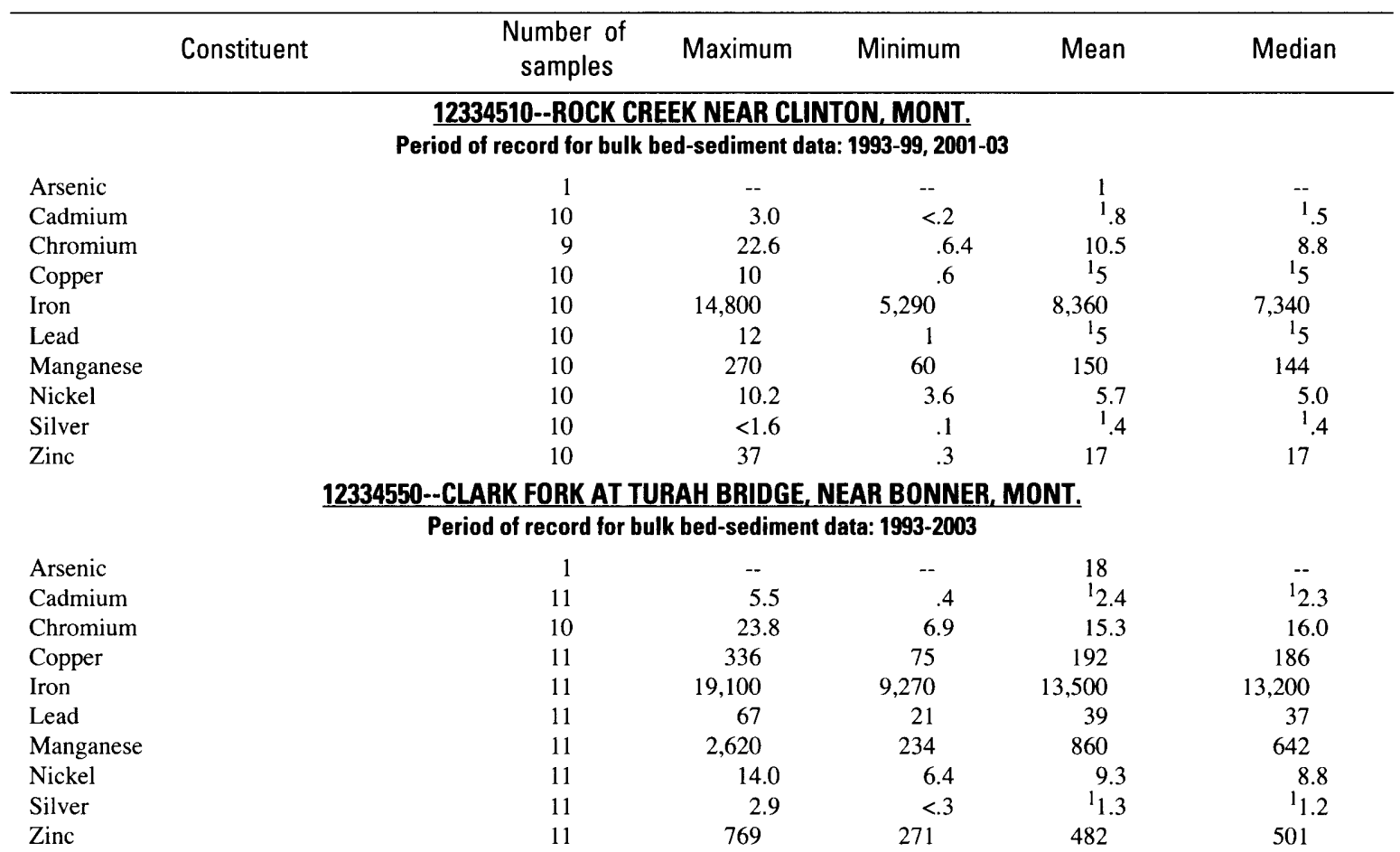

12340000--BLACKFOOT RIVER NEAR BONNER, MONT.

Period of record for bulk bed-sediment data: 1993-94, 1999-2001, 2003

Arsenic

Cadmium

Chromium

Copper

Iron

Lead

Manganese

Nickel

Silver

Zinc

Arsenic

Cadmium

Chromium

Copper

Iron

Lead

Manganese

Nickel

Silver

Zinc

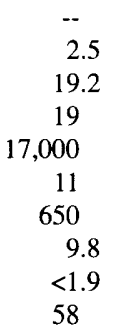
$<.2$
6.7
6
10,300
5
179
7.5
$<.4$
23

$$
\begin{gathered}
1-- \\
<1.5 \\
12.5 \\
15 \\
13,800 \\
9 \\
316 \\
9.0 \\
1.6
\end{gathered}
$$$$
35
$$

12340500--CLARK FORK ABOVE MISSOULA, MONT.

Period of record for bulk bed-sediment data: 1997-2003

$\begin{array}{cc}-- & -- \\ 5.2 & <.8 \\ 31.5 & 9.7 \\ 630 & 43 \\ 21,500 & 11,100 \\ 84 & 7 \\ 888 & 228 \\ 14.4 & 8.1 \\ 3.4 & <.4 \\ 1,210 & 145\end{array}$

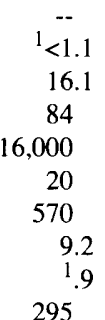


90 Water-Quality, Bed-Sediment, and Biological Data (October 2002 through September 2003) and Statistical Summaries of Data for Streams in the Upper Clark Fork Basin, Montana

Table 23. Statistical summary of bulk bed-sediment data for the upper Clark Fork basin, Montana, August 1993 through September 2003-Continued

\begin{tabular}{|c|c|c|c|c|c|}
\hline Constituent & $\begin{array}{c}\text { Number of } \\
\text { samples }\end{array}$ & Maximum & Minimum & Mean & Median \\
\hline \multicolumn{6}{|c|}{ 12353000--CLARK FORK BELOW MISSOULA, MONT ${ }^{2}$} \\
\hline \multicolumn{6}{|c|}{ Period of record for bulk bed-sediment data: 1993-2003 } \\
\hline Arsenic & 1 & -- & -- & 1.1 & -- \\
\hline Cadmium & 11 & 3.0 & $<.2$ & ${ }^{1} 1.0$ & ${ }^{1}<1.1$ \\
\hline Chromium & 10 & 12.7 & 4.4 & 7.7 & 7.1 \\
\hline Copper & 11 & 77 & 10 & 38 & 30 \\
\hline Iron & 11 & 13,300 & 5,830 & 8,910 & 8,830 \\
\hline Lead & 11 & 23 & 5 & ${ }^{1} 10$ & ${ }^{18}$ \\
\hline Manganese & 11 & 560 & 150 & 338 & 364 \\
\hline Nickel & 11 & 8.1 & 3.5 & 5.4 & 4.9 \\
\hline Silver & 11 & $<1.9$ & $<.3$ & ${ }^{1} .5$ & 1.5 \\
\hline Zinc & 11 & 183 & 39 & 110 & 101 \\
\hline
\end{tabular}

${ }^{1}$ Value determined by arbitrarily substituting one-half of the analytical detection limit for censored $(<)$ values when both uncensored and censored values were used in determining the mean and (or) median. When all data were less than the analytical detection level, the median was determined by ranking the censored values in order of magnitude.

${ }^{2}$ Samples collected about 30 miles downstream from streamflow-gaging station to conform to previous sampling location. 
Table 24. Statistical summary of biological data for the upper Clark Fork basin, Montana, August 1986 through September 2003

[Concentrations are in micrograms per gram dry weight. Number of composite samples represents the total of all individual composite samples collected for every year that the constituent was analyzed. Values for single samples are arbitrarily listed in the "Mean" column. Because Hydropsyche insects were not sorted to the species level during 1986-89, Hydropsyche species statistics for stations sampled during those years are based on the results of all Hydropsyche species combined. At some sites, statistics for the Hydropsyche morosa group are based on the combined results for two or more species. Insects collected during 1986-98 were depurated prior to analysis; depuration was discontinued in 1999. Abbreviation: spp., species. Symbols: <, less than analytical detection limit; --, indicates either too few samples (less than three) or insufficient data to compute statistic, or element not analyzed]

\begin{tabular}{|c|c|c|c|c|c|}
\hline Constituent & $\begin{array}{c}\text { Number of } \\
\text { composite } \\
\text { samples }\end{array}$ & Maximum & Minimum & Mean & Median \\
\hline \multicolumn{6}{|c|}{ 12323600--SILVER BOW CREEK AT OPPORTUNITY, MONT. } \\
\hline \multicolumn{6}{|c|}{ Period of record for biological data: 1992, 1994-95, 1997-2003 } \\
\hline \multicolumn{6}{|c|}{ Brachycentrus spp. } \\
\hline Cadmium & 5 & 12.5 & 5.8 & 10.1 & 11.6 \\
\hline Chromium & 5 & 5.9 & .7 & 2.1 & .9 \\
\hline Copper & 5 & 846 & 235 & 587 & 592 \\
\hline Iron & 5 & 1,190 & 335 & 617 & 469 \\
\hline Lead & 5 & 21.5 & 7.4 & 13.7 & 13.8 \\
\hline Manganese & 5 & 817 & 231 & 515 & 503 \\
\hline Nickel & 5 & 2.1 & $<.1$ & ${ }^{1} 1.3$ & ${ }^{1} 1.6$ \\
\hline Zinc & 5 & 995 & 629 & 803 & 815 \\
\hline \multicolumn{6}{|c|}{ Hydropsyche cockerelli } \\
\hline Arsenic & 1 & -- & -- & 20.4 & -- \\
\hline Cadmium & 7 & 9.7 & 4.1 & 6.0 & 5.2 \\
\hline Chromium & 7 & 8.0 & 1.0 & 3.6 & 3.1 \\
\hline Copper & 7 & 1,090 & 269 & 485 & 439 \\
\hline Iron & 7 & 2,660 & 689 & 1,300 & 1,000 \\
\hline Lead & 7 & 47.2 & 19.0 & 26.9 & 21.0 \\
\hline Manganese & 7 & 3,030 & 180 & 928 & 591 \\
\hline Nickel & 7 & 3.6 & .7 & 2.1 & 2.1 \\
\hline Zinc & 7 & 1,590 & 749 & 1,010 & 871 \\
\hline \multicolumn{6}{|c|}{ Hydropsyche spp. } \\
\hline Arsenic & 3 & 23.1 & 18.4 & 20.1 & 18.9 \\
\hline Cadmium & 8 & 10.6 & 5.0 & 7.5 & 7.1 \\
\hline Chromium & 8 & 4.7 & .6 & 2.2 & 2.2 \\
\hline Copper & 8 & 930 & 352 & 655 & 652 \\
\hline Iron & 8 & 2,290 & 1,270 & 1,870 & 1,990 \\
\hline Lead & 8 & 50.8 & 34.7 & 39.5 & 38.2 \\
\hline Manganese & 8 & 1,340 & 712 & 1,080 & 1,060 \\
\hline Nickel & 8 & 2.5 & 1.6 & 2.3 & 2.4 \\
\hline Zinc & 8 & 1,290 & 1,070 & 1,120 & 1,100 \\
\hline \multicolumn{6}{|c|}{ Hydropsyche tana } \\
\hline Cadmium & 6 & 9.2 & 4.8 & 6.8 & 6.9 \\
\hline Chromium & 6 & 11.5 & .9 & 4.5 & 1.8 \\
\hline Copper & 6 & 456 & 10.5 & 236 & 298 \\
\hline Iron & 6 & 1,520 & 857 & 1,100 & 1,050 \\
\hline Lead & 6 & 21.0 & 15.6 & 18.6 & 18.3 \\
\hline Manganese & 6 & 969 & 307 & 634 & 675 \\
\hline Nickel & 6 & 1.8 & .7 & 1.4 & 1.6 \\
\hline Zinc & 6 & 1,070 & 760 & 961 & 1,020 \\
\hline
\end{tabular}


92 Water-Quality, Bed-Sediment, and Biological Data (October 2002 through September 2003) and Statistical Summaries of Data for Streams in the Upper Clark Fork Basin, Montana

Table 24. Statistical summary of biological data for the upper Clark Fork basin, Montana, August 1986 through September 2003-Continued

\begin{tabular}{cccccc}
\hline Constituent & $\begin{array}{c}\text { Number of } \\
\text { composite } \\
\text { samples }\end{array}$ & Maximum & Minimum & Mean & Median \\
\hline
\end{tabular}

12323750--SILVER BOW CREEK AT WARM SPRINGS, MONT. Period of record for biological data: $1992-2003$

Arsenic

Cadmium

Chromium

Copper

Iron

Lead

Manganese

Nickel

Zinc

Arsenic

Cadmium

Chromium

Copper

Iron

Lead

Manganese

Nickel

Zinc

Cadmium

Chromium

Copper

Iron

Lead

Manganese

Nickel

Zinc

\section{Hydropsyche cockerelli}

2

28

28

28

28

28

28

28

28

\section{Hydropsyche occidentalis}

17

17

17

17

17

17

17

17

26.8
1.6
6.8
48.9
2,960
8.2
3,540
2.7
211

Hydropsyche spp.

$\begin{array}{lcccc}4 & 2.3 & .4 & 1.1 & .9 \\ 4 & 1.4 & .5 & .8 & 1.2 \\ 4 & 47.6 & 34.9 & 40.9 & 40.6 \\ 4 & 773 & 561 & 680 & 693 \\ 4 & 5.1 & 1.9 & 2.9 & 4.7 \\ 4 & 1,100 & 443 & 725 & 678 \\ 4 & 1.9 & <.4 & 1.8 & 1.5 \\ 4 & 285 & 141 & 195 & 177\end{array}$

12323770--WARM SPRINGS CREEK AT WARM SPRINGS, MONT.

Period of record for biological data: 1995, 1997, 1999, 2002

Cadmium

Chromium

Copper

Iron

Lead

Manganese

Nickel

Zinc

\section{Arctopsyche grandis}

$\begin{array}{cc}3.0 & 1.9 \\ 2.9 & .8 \\ 102 & 78.3 \\ 1,040 & 684 \\ 5.6 & 3.0 \\ 3,560 & 1,340 \\ 2.3 & 1.8 \\ 222 & 181\end{array}$

2.4
1.7
93.7
839
$1_{4.3}$
2,250
$1_{2.1}$
196

2.2

97.2

815

4.3

2,040

2.2

190 
Table 24. Statistical summary of biological data for the upper Clark Fork basin, Montana, August 1986 through September 2003-Continued

\begin{tabular}{|c|c|c|c|c|c|c|}
\hline & Constituent & $\begin{array}{c}\text { Number of } \\
\text { composite } \\
\text { samples }\end{array}$ & Maximum & Minimum & Mean & Median \\
\hline \multicolumn{7}{|c|}{ 12323770--WARM SPRINGS CREEK AT WARM SPRINGS, MONT,-Continued } \\
\hline \multicolumn{7}{|c|}{ Period of record for biological data: 1995, 1997, 1999, 2002} \\
\hline \multicolumn{7}{|c|}{ Hydropsyche occidentalis } \\
\hline Cadmium & & 2 & .8 & .7 & .8 & -- \\
\hline Chromium & & 2 & 3.2 & 3.2 & 3.2 & -- \\
\hline Copper & & 2 & 183 & 181 & 182 & -- \\
\hline Iron & & 2 & 2,070 & 1,950 & 2,010 & -- \\
\hline Lead & & 2 & 8.2 & 6.7 & 7.4 & -- \\
\hline Manganese & & 2 & 2,480 & 2,400 & 2,440 & -- \\
\hline Nickel & & 2 & 3.3 & 3.0 & 3.2 & -- \\
\hline Zinc & & 2 & 172 & 166 & 169 & -- \\
\hline \multicolumn{7}{|c|}{ Hydropsyche spp. } \\
\hline Cadmium & & 2 & 1.1 & .6 & .9 & -- \\
\hline Chromium & & 2 & 1.6 & 1.4 & 1.5 & -- \\
\hline Copper & & 2 & 95.9 & 94.8 & 95.3 & -- \\
\hline Iron & & 2 & 1,220 & 1,150 & 1,190 & -- \\
\hline Lead & & 2 & 5.9 & 5.2 & 5.6 & -- \\
\hline Manganese & & 2 & 3,390 & 956 & 2,170 & -- \\
\hline Nickel & & 2 & 2.0 & 1.8 & 1.9 & -- \\
\hline Zinc & & 2 & 129 & 125 & 127 & -- \\
\hline
\end{tabular}

12323800--CLARK FORK NEAR GALEN, MONT.

Period of record for biological data: 1987, 1991-2003

Arsenic

Cadmium

Chromium

Copper

Iron

Lead

Manganese

Nickel

Zinc

Cadmium

Chromium

Copper

Iron

Lead

Manganese

Nickel

Zinc

Arsenic

Cadmium

Chromium

Copper

Iron

Lead

Manganese

Nickel

Zinc

Hydropsyche cockerelli

$\begin{array}{lcccc}1 & - & - & 13.6 & -- \\ 26 & 2.7 & .7 & 1.5 & 1.5 \\ 26 & 4.4 & .8 & 1.8 & 1.6 \\ 26 & 181 & 48.7 & 98.0 & 95.0 \\ 26 & 2,460 & 816 & 1,380 & 1,340 \\ 26 & 11.7 & 1.2 & 7.5 & 7.6 \\ 26 & 3,620 & 1,070 & 2,240 & 2,250 \\ 26 & 3.1 & .9 & 1.6 & 1.4 \\ 26 & 299 & 136 & 210 & 208 \\ \text { Hydropsyche morosa group } & & & \\ 5 & 3.2 & 2.4 & 2.5 & 2.4 \\ 5 & 4.6 & 1.8 & 2.6 & 2.2 \\ 5 & 185 & 156 & 173 & 175 \\ 5 & 1,890 & 1,360 & 1,510 & 1,430 \\ 5 & 12.4 & 7.1 & 8.5 & 7.9 \\ 5 & 3,960 & 2,360 & 3,500 & 3,860 \\ 5 & 3.6 & 1.9 & 2.3 & 2.1 \\ 5 & 349 & 292 & 309 & 303\end{array}$

\section{Hydropsyche occidentalis}

$\begin{array}{rcccc}3 & 16.5 & 15.9 & 16.1 & 16.1 \\ 35 & 1.7 & .6 & 1.1 & 1.0 \\ 35 & 6.6 & .4 & 2.0 & 1.5 \\ 35 & 121 & 49.2 & 82.0 & 80.8 \\ 35 & 1,920 & 642 & 1,260 & 1,200 \\ 35 & 13.5 & 1.6 & 7.0 & 6.5 \\ 35 & 6,170 & 1,220 & 2,560 & 2,240 \\ 35 & 3.5 & .8 & 1.6 & 1.5 \\ 35 & 286 & 168 & 199 & 191\end{array}$


94 Water-Quality, Bed-Sediment, and Biological Data (October 2002 through September 2003) and Statistical Summaries of Data for Streams in the Upper Clark Fork Basin, Montana

Table 24. Statistical summary of biological data for the upper Clark Fork basin, Montana, August 1986 through September 2003-Continued

\begin{tabular}{|c|c|c|c|c|c|}
\hline Constituent & $\begin{array}{c}\text { Number of } \\
\text { composite } \\
\text { samples }\end{array}$ & Maximum & Minimum & Mean & Median \\
\hline \multicolumn{6}{|c|}{ 12323800--CLARK FORK NEAR GALEN, MONT_-Continued } \\
\hline \multicolumn{6}{|c|}{ Hydropsyche tana } \\
\hline Cadmium & 1 & -- & -- & 1.5 & -- \\
\hline Chromium & 1 & -- & -- & 1.4 & -- \\
\hline Copper & 1 & -- & -- & 92.9 & -- \\
\hline Iron & 1 & -- & -- & 1,340 & -- \\
\hline Lead & 1 & -- & -- & 9.0 & -- \\
\hline Manganese & 1 & -- & -- & 2,160 & -- \\
\hline Nickel & 1 & -- & -- & 2.1 & -- \\
\hline Zinc & 1 & -- & -- & 206 & -- \\
\hline \multicolumn{6}{|c|}{ Hydropsyche spp. } \\
\hline Cadmium & 4 & 3.5 & 2.6 & 3.0 & 3.0 \\
\hline Chromium & 0 & -- & -- & -- & -- \\
\hline Copper & 4 & 154 & 135 & 148 & 152 \\
\hline Iron & 4 & 1,540 & 1,190 & 1,400 & 1,450 \\
\hline Lead & 4 & 13.5 & 10.5 & 12.2 & 12.4 \\
\hline Manganese & 0 & -- & -. & -- & -- \\
\hline Nickel & 0 & -- & -- & -- & -- \\
\hline Zinc & 4 & 329 & 279 & 308 & 313 \\
\hline
\end{tabular}

461415112450801--CLARK FORK BELOW LOST CREEK, NEAR GALEN, MONT.

Period of record for biological data: 1996-2003

Arsenic

Cadmium

Chromium

Copper

Iron

Lead

Manganese

Nickel

Zinc

Arsenic

Cadmium

Chromium

Copper

Iron

Lead

Manganese

Nickel

Zinc

Arsenic

Cadmium

Chromium

Copper

Iron

Lead
Claassenia sabulosa

\begin{tabular}{|c|c|c|c|c|}
\hline 1 & -- & -- & 1.5 & -- \\
\hline 2 & .4 & .3 & .4 & -- \\
\hline 2 & 1.9 & .4 & 1.2 & -- \\
\hline 2 & 70.1 & 67.1 & 68.6 & -- \\
\hline 2 & 209 & 189 & 199 & -- \\
\hline 2 & 1.2 & .7 & 1.0 & -- \\
\hline 2 & 238 & 90.4 & 164 & -- \\
\hline 2 & 1.9 & $<.2$ & ${ }^{1} 1.0$ & -- \\
\hline 2 & 245 & 208 & 226 & -- \\
\hline \multicolumn{5}{|c|}{ Hydropsyche cockerelli } \\
\hline 3 & 11.8 & 11.2 & 11.5 & 11.6 \\
\hline 14 & 2.8 & 1.1 & 1.8 & 1.9 \\
\hline 14 & 2.7 & .8 & 2.0 & 2.2 \\
\hline 14 & 147 & 48.8 & 104 & 97.0 \\
\hline 14 & 2,570 & 691 & 1,330 & 1,180 \\
\hline 14 & 15.2 & 4.5 & 10.3 & 9.8 \\
\hline 14 & 3,160 & 1,230 & 1,890 & 1,700 \\
\hline 14 & 1.9 & .9 & 1.3 & 1.1 \\
\hline 14 & 321 & 151 & 214 & 226 \\
\hline \multicolumn{5}{|c|}{ Hydropsyche occidentalis } \\
\hline 2 & 12.9 & 12.7 & 12.8 & -. \\
\hline 16 & 1.8 & .9 & 1.3 & 1.5 \\
\hline 16 & 3.3 & 1.3 & 2.0 & 2.0 \\
\hline 16 & 157 & 52.1 & 104 & 98.2 \\
\hline 16 & 1,920 & 963 & 1,380 & 1,270 \\
\hline 6 & 12.4 & 6.6 & 9.6 & 9.8 \\
\hline
\end{tabular}


Table 24. Statistical summary of biological data for the upper Clark Fork basin, Montana, August 1986 through September 2003-Continued

\begin{tabular}{|c|c|c|c|c|c|c|}
\hline & Constituent & $\begin{array}{c}\text { Number of } \\
\text { composite } \\
\text { samples }\end{array}$ & Maximum & Minimum & Mean & Median \\
\hline \multicolumn{7}{|c|}{ 461415112450801--CLARK FORK BELOW LOST CREEK, NEAR GALEN, MONT.-Continued } \\
\hline \multicolumn{7}{|c|}{ Period of record for biological data: 1996-2003 } \\
\hline \multicolumn{7}{|c|}{ Hydropsyche occidentalis - Continued } \\
\hline Manganese & & 16 & 3,440 & 1,220 & 2,150 & 1,850 \\
\hline Nickel & & 16 & 1.7 & .9 & 1.3 & 1.3 \\
\hline Zinc & & 16 & 283 & 174 & 224 & 226 \\
\hline \multicolumn{7}{|c|}{ Hydropsyche spp. } \\
\hline Arsenic & & 1 & -- & -- & 12.0 & -- \\
\hline Cadmium & & 5 & 1.8 & 1.2 & 1.5 & 1.4 \\
\hline Chromium & & 5 & 2.4 & .9 & 1.5 & 1.5 \\
\hline Copper & & 5 & 122 & 45.1 & 91.8 & 103 \\
\hline Iron & & 5 & 1,410 & 533 & 1,110 & 1,200 \\
\hline Lead & & 5 & 20.5 & 4.1 & 10.0 & 8.7 \\
\hline Manganese & & 5 & 1,980 & 799 & 1,440 & 1,230 \\
\hline Nickel & & 5 & 2.8 & 1.0 & 1.6 & 1.4 \\
\hline Zinc & & 5 & 225 & 143 & 179 & 179 \\
\hline
\end{tabular}

461559112443301--CLARK FORK NEAR RACETRACK, MONT.

Period of record for biological data: 1996-2003

Cadmium

Chromium

Copper

Iron

Lead

Manganese

Nickel

Zinc

Arsenic

Cadmium

Chromium

Copper

Iron

Lead

Manganese

Nickel

Zinc

Arsenic

Cadmium

Chromium

Copper

Iron

Lead

Manganese

Nickel

Zinc

\section{Claassenia sabulosa}

\begin{tabular}{|c|c|c|c|c|}
\hline 1 & -- & -- & .4 & -- \\
\hline 1 & -- & -- & .3 & -- \\
\hline 1 & -- & -- & 40.3 & -- \\
\hline 1 & -- & -. & 113 & -- \\
\hline 1 & -- & -- & .8 & -- \\
\hline 1 & -- & -- & 172 & -- \\
\hline 1 & -- & -. & .2 & -- \\
\hline 1 & -- & -- & 213 & -- \\
\hline \multicolumn{5}{|c|}{ Hydropsyche cockerelli } \\
\hline 2 & 11.7 & 11.5 & 11.6 & -- \\
\hline 3 & 1.9 & 1.0 & 1.4 & 1.4 \\
\hline & 2.7 & .6 & 1.6 & 1.4 \\
\hline 3 & 109 & 50.0 & 78.2 & 74.1 \\
\hline 3 & 1,370 & 657 & 955 & 981 \\
\hline 3 & 10.5 & 3.7 & 6.7 & 6.4 \\
\hline & 2,010 & 646 & 1,390 & 1,530 \\
\hline & 1.4 & .7 & 1.0 & 1.0 \\
\hline & 199 & 139 & 172 & 171 \\
\hline \multicolumn{5}{|c|}{ Hydropsyche occidentalis } \\
\hline 2 & 14.3 & 13.7 & 14.0 & -- \\
\hline 5 & 2.2 & .7 & 1.4 & 1.4 \\
\hline 5 & 3.7 & 1.1 & 2.1 & 2.0 \\
\hline 5 & 160 & 59.5 & 107 & 107 \\
\hline 5 & 1,880 & 1,030 & 1,510 & 1,520 \\
\hline & 11.7 & 4.3 & 9.6 & 10.1 \\
\hline$J$ & 3,770 & 1,090 & 2,070 & 2,050 \\
\hline & 1.9 & 1.1 & 1.3 & 1.3 \\
\hline & 255 & 181 & 225 & 220 \\
\hline
\end{tabular}


96 Water-Quality, Bed-Sediment, and Biological Data (October 2002 through September 2003) and Statistical Summaries of Data for Streams in the Upper Clark Fork Basin, Montana

Table 24. Statistical summary of biological data for the upper Clark Fork basin, Montana, August 1986 through September 2003-Continued

\begin{tabular}{|c|c|c|c|c|c|c|}
\hline & Constituent & $\begin{array}{c}\text { Number of } \\
\text { composite } \\
\text { samples }\end{array}$ & Maximum & Minimum & Mean & Median \\
\hline \multicolumn{7}{|c|}{$\frac{\text { 461559112443301--CLARK FORK NEAR RACETRACK, MONT.-Continued }}{\text { Period of record for biological data: } 1996-2003}$} \\
\hline \multicolumn{7}{|c|}{ Hydropsyche spp. } \\
\hline Arsenic & & 1 & -- & -- & 11.9 & -- \\
\hline Cadmium & & 3 & 2.4 & 1.0 & 1.6 & 1.5 \\
\hline Chromium & & 3 & 1.7 & .7 & 1.2 & 1.1 \\
\hline Copper & & 3 & 113 & 82.9 & 93.7 & 85.2 \\
\hline Iron & & 3 & 1,290 & 1,140 & 1,210 & 1,200 \\
\hline Lead & & 3 & 9.6 & 5.7 & 7.5 & 7.4 \\
\hline Manganese & & 3 & 1,600 & 910 & 1,210 & 1,130 \\
\hline Nickel & & 3 & 1.4 & 1.1 & 1.3 & 1.3 \\
\hline Zinc & & 3 & 208 & 151 & 180 & 181 \\
\hline
\end{tabular}

461903112440701--CLARK FORK AT DEMPSEY CREEK DIVERSION, NEAR RACETRACK, MONT. Period of record for biological data: 1996-2003

Cadmium
Chromium
Copper
Iron
Lead
Manganese
Nickel
Zinc

Arsenic
Cadmium
Chromium
Copper
Iron
Lead
Manganese
Nickel
Zinc

Arsenic
Cadmium
Chromium
Copper
Iron
Lead
Manganese
Nickel
Zinc

\section{Arctopsyche grandis}

$\begin{array}{ll}1 & -- \\ 1 & -- \\ 1 & -- \\ 1 & -- \\ 1 & -- \\ 1 & -- \\ 1 & -- \\ 1 & --\end{array}$

$\begin{array}{lc}-- & 1.7 \\ -- & <2.4 \\ -- & 30.8 \\ -- & 340 \\ -- & <14.5 \\ -- & 510 \\ -- & 1.0 \\ -- & 87\end{array}$

\section{Hydropsyche cockerelli}

$\begin{array}{lcccc}1 & -- & - & 18.8 & -- \\ 0 & 1.6 & .7 & 1.2 & 1.3 \\ 0 & 4.0 & .4 & 1.3 & 1.0 \\ 0 & 190 & 60.7 & 91.3 & 76.7 \\ 0 & 2,310 & 552 & 973 & 841 \\ 0 & 17.7 & 3.5 & 7.1 & 5.9 \\ 0 & 1,530 & 487 & 1,010 & 1,140 \\ 0 & 1.9 & .5 & 1.0 & .8 \\ 0 & 275 & 162 & 187 & 179 \\ \text { Hydropsyche occidentalis } & & & \\ 1 & -- & -- & 24.0 & -- \\ 8 & 1.8 & .7 & 1.2 & 1.1 \\ 8 & 6.2 & .8 & 2.1 & 1.8 \\ 8 & 238 & 74.9 & 103 & 88.3 \\ 8 & 3,390 & 940 & 1,520 & 1,500 \\ 8 & 21.8 & 6.1 & 11.6 & 11.4 \\ 8 & 3,990 & 826 & 2,420 & 2,280 \\ 8 & 2.4 & 1.2 & 1.5 & 1.4 \\ 8 & 355 & 222 & 252 & 236\end{array}$


Table 24. Statistical summary of biological data for the upper Clark Fork basin, Montana, August 1986 through September 2003-Continued

\begin{tabular}{cccccc}
\hline Constituent & $\begin{array}{c}\text { Number of } \\
\text { composite } \\
\text { samples }\end{array}$ & Maximum & Minimum & Mean & Median \\
\hline
\end{tabular}

\begin{tabular}{|c|c|c|c|c|c|}
\hline \multicolumn{6}{|c|}{$\begin{array}{l}\text { 461903112440701--CLARK FORK AT DEMPSEY CREEK DIVERSION, NEAR RACETRACK, MONT.-Continued } \\
\text { Period of record for biological data: 1996-2003 }\end{array}$} \\
\hline \multicolumn{6}{|c|}{ Hydropsyche spp. } \\
\hline Cadmium & 2 & 1.7 & 1.6 & 1.6 & -- \\
\hline Chromium & 2 & 2.1 & 1.4 & 1.8 & -- \\
\hline Copper & 2 & 140 & 104 & 122 & -- \\
\hline Iron & 2 & 1,610 & 1,070 & 1,340 & -- \\
\hline Lead & 2 & 13.2 & 10.5 & 11.8 & -- \\
\hline Manganese & 2 & 1,150 & 638 & 892 & -- \\
\hline Nickel & 2 & 1.6 & 1.6 & 1.6 & -- \\
\hline Zinc & 2 & 212 & 191 & 202 & -- \\
\hline
\end{tabular}

12324200--CLARK FORK AT DEER LODGE, MONT.

Period of record for biological data: 1986-87, 1990-2003

\begin{tabular}{|c|c|c|c|c|c|}
\hline \multicolumn{6}{|c|}{ Arctopsyche grandis } \\
\hline Cadmium & 2 & 2.4 & $<4.2$ & ${ }^{1} 2.2$ & -- \\
\hline Chromium & 2 & 1.0 & $<1.3$ & ${ }^{1} .8$ & -- \\
\hline Copper & 2 & 69.1 & 34.9 & 52.0 & -- \\
\hline Iron & 2 & 676 & 537 & 606 & -- \\
\hline Lead & 2 & $<7.8$ & 3.8 & $1_{3.8}$ & -- \\
\hline Manganese & 2 & 727 & 380 & 554 & -- \\
\hline Nickel & 2 & $<1.7$ & $<1.3$ & ${ }^{1}-$ & -- \\
\hline Zinc & 2 & 178 & 140 & 159 & -- \\
\hline \multicolumn{6}{|c|}{ Hydropsyche cockerelli } \\
\hline Arsenic & 1 & -- & -- & 10.1 & -- \\
\hline Cadmium & 24 & 2.3 & .6 & 1.4 & 1.3 \\
\hline Chromium & 24 & 3.2 & .4 & 1.6 & 1.7 \\
\hline Copper & 24 & 136 & 54.7 & 94.5 & 98.0 \\
\hline Iron & 24 & 3,340 & 490 & 1,140 & 1,050 \\
\hline Lead & 24 & 18.2 & 4.3 & 9.7 & 8.9 \\
\hline Manganese & 24 & 1,490 & 396 & 817 & 710 \\
\hline Nickel & 24 & 2.4 & .3 & 1.2 & 1.0 \\
\hline Zinc & 24 & 391 & 132 & 186 & 188 \\
\hline \multicolumn{6}{|c|}{ Hydropsyche occidentalis } \\
\hline Arsenic & 2 & 12.4 & 11.3 & 11.8 & -- \\
\hline Cadmium & 39 & 2.7 & .8 & 1.3 & 1.3 \\
\hline Chromium & 39 & 3.6 & .6 & 1.9 & 1.9 \\
\hline Copper & 39 & 162 & 49.4 & 113 & 110 \\
\hline Iron & 39 & 2,060 & 557 & 1,420 & 1,430 \\
\hline Lead & 39 & 18.6 & 3.5 & 11.0 & 10.8 \\
\hline Manganese & 39 & 2,840 & 649 & 1,670 & 1,710 \\
\hline Nickel & 39 & 12.9 & 1.0 & 1.8 & 1.4 \\
\hline Zinc & 39 & 329 & 166 & 237 & 231 \\
\hline \multicolumn{6}{|c|}{ Hydropsyche spp. } \\
\hline Cadmium & 3 & 2.0 & 1.2 & 1.6 & 1.6 \\
\hline Chromium & 0 & -- & -- & -- & -- \\
\hline Copper & 3 & 222 & 103 & 145 & 111 \\
\hline Iron & 3 & 2,220 & 1,110 & 1,520 & 1,240 \\
\hline Lead & 3 & 15.0 & 5.6 & 8.8 & 5.7 \\
\hline Manganese & 0 & -- & -- & -- & -- \\
\hline
\end{tabular}


98 Water-Quality, Bed-Sediment, and Biological Data (October 2002 through September 2003) and Statistical Summaries of Data for Streams in the Upper Clark Fork Basin, Montana

Table 24. Statistical summary of biological data for the upper Clark Fork basin, Montana, August 1986 through September 2003-Continued

\begin{tabular}{|c|c|c|c|c|c|}
\hline Constituent & $\begin{array}{c}\text { Number of } \\
\text { composite } \\
\text { samples }\end{array}$ & Maximum & Minimum & Mean & Median \\
\hline \multicolumn{6}{|c|}{ 12324200--CLARK FORK AT DEER LODGE, MONT-Continued } \\
\hline \multicolumn{6}{|c|}{ Hydropsyche spp.-Continued } \\
\hline Nickel & 0 & -- & -- & -- & -- \\
\hline Zinc & 3 & 203 & 185 & 195 & 197 \\
\hline \multicolumn{6}{|c|}{ 12324590--LITTLE BLACKFOOT RIVER NEAR GARRISON, MONT. } \\
\hline \multicolumn{6}{|c|}{ Period of record for biological data: 1987, 1994, 1998, 2001} \\
\hline \multicolumn{6}{|c|}{ Arctopsyche grandis } \\
\hline Cadmium & 15 & .7 & .2 & .4 & .4 \\
\hline Chromium & 15 & 1.6 & 6 & .9 & .8 \\
\hline Copper & 15 & 14.2 & 9.0 & 12.2 & 13.0 \\
\hline Iron & 15 & 677 & 177 & 384 & 313 \\
\hline Lead & 15 & 1.3 & .5 & .8 & .8 \\
\hline Manganese & 15 & 1,140 & 318 & 678 & 551 \\
\hline Nickel & 15 & 1.4 & .4 & .7 & .6 \\
\hline Zinc & 15 & 214 & 113 & 165 & 162 \\
\hline \multicolumn{6}{|c|}{ Claassenia sabulosa } \\
\hline Cadmium & 7 & .5 & .1 & .2 & .2 \\
\hline Chromium & 7 & .9 & .3 & 6 & .7 \\
\hline Copper & 7 & 36.1 & 20.0 & 29.4 & 30.3 \\
\hline Iron & 7 & 319 & 98 & 175 & 156 \\
\hline Lead & 7 & $<.8$ & $<.1$ & ${ }^{1} .4$ & ${ }^{1} .3$ \\
\hline Manganese & 7 & 90.5 & 46.7 & 63.8 & 61.6 \\
\hline Nickel & 7 & .7 & .4 & .5 & .5 \\
\hline Zinc & 7 & 233 & 172 & 203 & 202 \\
\hline \multicolumn{6}{|c|}{ Hydropsyche cockerelli } \\
\hline Cadmium & 1 & -- & -- & .6 & -- \\
\hline Chromium & 1 & -- & -- & 1.6 & -- \\
\hline Copper & 1 & -- & -- & 28.4 & -- \\
\hline Iron & 1 & -- & -- & 478 & -- \\
\hline Lead & 1 & -- & -- & 3.6 & -- \\
\hline Manganese & 1 & -- & -- & 399 & -- \\
\hline Nickel & 1 & -- & -- & 1.2 & -- \\
\hline Zinc & 1 & -- & -- & 123 & -- \\
\hline \multicolumn{6}{|c|}{ Hydropsyche occidentalis } \\
\hline Cadmium & 2 & $<.7$ & .3 & 1.3 & -- \\
\hline Chromium & 2 & 2.3 & 1.3 & 1.8 & -- \\
\hline Copper & 2 & 15.2 & 15.1 & 15.2 & -- \\
\hline Iron & 2 & 1,340 & 426 & 883 & -- \\
\hline Lead & 2 & 2.3 & $<3.7$ & ${ }^{1} 2.1$ & -- \\
\hline Manganese & 2 & 554 & 434 & 494 & -- \\
\hline Nickel & 2 & 1.1 & .8 & 1.0 & -- \\
\hline Zinc & 2 & 137 & 110 & 124 & -- \\
\hline
\end{tabular}


Table 24. Statistical summary of biological data for the upper Clark Fork basin, Montana, August 1986 through September 2003-Continued

\begin{tabular}{|c|c|c|c|c|c|}
\hline Constituent & $\begin{array}{l}\text { Number of } \\
\text { composite } \\
\text { samples }\end{array}$ & Maximum & Minimum & Mean & Median \\
\hline Period of record for biological data: $1992-2003$ & \multicolumn{5}{|c|}{ 12324680--CLARK FORK AT GOLDCREEK, MONT. } \\
\hline \multicolumn{6}{|c|}{ Arctopsyche grandis } \\
\hline Arsenic & 6 & 6.4 & 5.8 & 6.1 & 6.1 \\
\hline Cadmium & 35 & 6.6 & .6 & 2.2 & 2.0 \\
\hline Chromium & 35 & 3.3 & .4 & 1.4 & 1.1 \\
\hline Copper & 35 & 129 & 21.4 & 48.0 & 40.8 \\
\hline Iron & 35 & 2,360 & 339 & 769 & 624 \\
\hline Lead & 35 & 10.9 & 2.0 & 4.0 & 3.6 \\
\hline Manganese & 35 & 1,580 & 592 & 851 & 803 \\
\hline Nickel & 35 & 1.8 & .2 & .8 & .7 \\
\hline Zinc & 35 & 326 & 149 & 204 & 186 \\
\hline \multicolumn{6}{|c|}{$\underline{\text { Claassenia sabulosa }}$} \\
\hline Arsenic & 3 & 1.0 & .7 & .9 & .9 \\
\hline Cadmium & 23 & 3.5 & .3 & 1.2 & .8 \\
\hline Chromium & 23 & 1.6 & .2 & .6 & .5 \\
\hline Copper & 23 & 81.7 & 33.0 & 56.7 & 56.3 \\
\hline Iron & 23 & 567 & 63.0 & 192 & 171 \\
\hline Lead & 23 & 1.8 & .4 & 1.0 & 1.0 \\
\hline Manganese & 23 & 279 & 50.6 & 123 & 106 \\
\hline Nickel & 23 & .7 & .1 & .3 & .3 \\
\hline Zinc & 23 & 351 & 166 & 263 & 261 \\
\hline \multicolumn{6}{|c|}{ Hydropsyche cockerelli } \\
\hline Arsenic & 4 & 6.1 & 5.7 & 5.9 & 6.0 \\
\hline Cadmium & 23 & 2.6 & .5 & 1.4 & 1.3 \\
\hline Chromium & 23 & 4.7 & .5 & 2.2 & 2.0 \\
\hline Copper & 23 & 188 & 17.1 & 73.5 & 58.6 \\
\hline Iron & 23 & 3,250 & 522 & 1,220 & 1,010 \\
\hline Lead & 23 & 16.2 & 2.4 & 7.2 & 5.5 \\
\hline Manganese & 23 & 1,670 & 538 & 862 & 900 \\
\hline Nickel & 23 & 2.3 & .3 & 1.2 & 1.2 \\
\hline Zinc & 23 & 249 & 106 & 183 & 186 \\
\hline \multicolumn{6}{|c|}{ Hydropsyche morosa group } \\
\hline Cadmium & 4 & 1.7 & 1.1 & 1.4 & 1.4 \\
\hline Chromium & 4 & 1.4 & 1.3 & 1.4 & 1.4 \\
\hline Copper & 4 & 72.9 & 43.8 & 60.5 & 62.7 \\
\hline Iron & 4 & 1,320 & 612 & 1,050 & 1,130 \\
\hline Lead & 4 & 6.9 & 2.4 & 4.6 & 4.6 \\
\hline Manganese & 4 & 1,030 & 538 & 804 & 822 \\
\hline Nickel & 4 & 1.4 & .9 & 1.2 & 1.2 \\
\hline Zinc & 4 & 190 & 137 & 167 & 170 \\
\hline \multicolumn{6}{|c|}{ Hydropsyche occidentalis } \\
\hline Arsenic & 1 & -- & -- & 5.8 & -- \\
\hline Cadmium & 16 & 1.7 & .5 & 1.2 & 1.3 \\
\hline Chromium & 16 & 3.9 & .4 & 1.6 & 1.7 \\
\hline Copper & 16 & 156 & 26.4 & 64.4 & 59.8 \\
\hline Iron & 16 & 2,720 & 466 & 1,140 & 1,070 \\
\hline Lead & 16 & 15.7 & 2.9 & 7.2 & 6.0 \\
\hline Manganese & 16 & 2,210 & 530 & 1,140 & 1,020 \\
\hline Nickel & 16 & 2.5 & .8 & 1.2 & 1.1 \\
\hline Zinc & 16 & 277 & 97 & 193 & 190 \\
\hline
\end{tabular}


100 Water-Quality, Bed-Sediment, and Biological Data (October 2002 through September 2003) and Statistical Summaries of Data for Streams in the Upper Clark Fork Basin, Montana

Table 24. Statistical summary of biological data for the upper Clark Fork basin, Montana, August 1986 through September 2003-Continued

\begin{tabular}{cccccc}
\hline Constituent & $\begin{array}{c}\text { Number of } \\
\text { composite } \\
\text { samples }\end{array}$ & Maximum Minimum Mean & Median \\
\hline
\end{tabular}

12331500--FLINT CREEK NEAR DRUMMOND, MONT.

Period of record for biological data: 1986, 1992-2003

Arsenic

Cadmium

Chromium

Copper

Iron

Lead

Manganese

Nickel

Zinc

Arsenic

Cadmium

Chromium

Copper

Iron

Lead

Manganese

Nickel

Zinc

Cadmium

Chromium

Copper

Iron

Lead

Manganese

Nickel

Zinc

Cadmium

Chromium

Copper

Iron

Lead

Manganese

Nickel

Zinc

Cadmium

Chromium

Copper

Iron

Lead

Manganese

Nickel

Zinc

Arctopsyche grandis

$\begin{array}{rcccc}3 & 9.0 & 7.4 & 8.1 & 7.8 \\ 42 & .8 & .1 & .4 & .4 \\ 42 & 7.1 & .3 & 2.1 & 1.7 \\ 42 & 22.2 & 8.9 & 15.2 & 15.3 \\ 42 & 2,460 & 412 & 1,360 & 1,360 \\ 42 & 17.5 & 3.7 & 8.9 & 8.2 \\ 42 & 3,160 & 424 & 1,570 & 1,400 \\ 42 & 2.7 & .6 & 1.4 & 1.3 \\ 42 & 275 & 93 & 199 & 195\end{array}$

\section{Hydropsyche cockerelli}

2

13

13

13

13

13

13

13

13

\section{Hydropsyche occidentalis}

\section{7}

7

7

7

7

7

7

7

Hydropsyche spp.

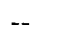

$--$

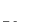

$--$

$--$

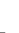

$-$

Hydropsyche tana

$<1.2$
10.3
16.0
1,320
15.3
1,400
3.1
139

$<.1$
.6
5.4
729
5.0
1,180
.5
107

16.8

.1
.9

9.5

996

3.1

401

.9

$$
17.0
$$

.5

2.9

18.2

2,440

15.5

1,590

2.2

177

195

0

1.3

$-$

.4

2.0

18.1

2,180

16.3

1,230

2.3

183

$\begin{array}{rcr}.2 & & \\ .7 & .6 & .6 \\ 15.1 & 2.5 & 2.1 \\ 912 & 1,900 & 18.6 \\ 5.8 & 19.4 & 1,870 \\ 1,400 & 2,270 & 24.0 \\ .8 & 3.0 & 1,780 \\ 128 & 185 & 2.4\end{array}$


Table 24. Statistical summary of biological data for the upper Clark Fork basin, Montana, August 1986 through September 2003-Continued

\begin{tabular}{|c|c|c|c|c|c|}
\hline Constituent & $\begin{array}{c}\text { Number of } \\
\text { composite } \\
\text { samples }\end{array}$ & Maximum & Minimum & Mean & Median \\
\hline \multicolumn{6}{|c|}{ 12331800-CLARK FORK NEAR DRUMMOND, MONT. } \\
\hline \multicolumn{6}{|c|}{ Period of record for biological data: 1986, 1991-2003 } \\
\hline \multicolumn{6}{|c|}{ Arctopsyche grandis } \\
\hline Arsenic & 3 & 3.6 & 3.2 & 3.3 & 3.3 \\
\hline Cadmium & 35 & 3.8 & .5 & 1.4 & 1.3 \\
\hline Chromium & 35 & 2.5 & .2 & 1.0 & 1.0 \\
\hline Copper & 35 & 89.2 & 16.9 & 33.8 & 28.2 \\
\hline Iron & 35 & 1,660 & 240 & 604 & 547 \\
\hline Lead & 35 & 11.8 & 2.1 & 4.6 & 4.1 \\
\hline Manganese & 35 & 2,010 & 462 & 840 & 733 \\
\hline Nickel & 35 & 1.9 & .2 & .7 & .6 \\
\hline Zinc & 35 & 308 & 140 & 190 & 189 \\
\hline \multicolumn{6}{|c|}{$\underline{\text { Claassenia sabulosa }}$} \\
\hline Arsenic & 2 & .7 & .7 & .7 & -- \\
\hline Cadmium & 38 & 2.8 & .2 & 1.1 & 1.1 \\
\hline Chromium & 38 & 3.3 & .1 & .7 & .5 \\
\hline Copper & 38 & 165 & 18.0 & 64.9 & 55.9 \\
\hline Iron & 38 & 387 & 45.4 & 159 & 135 \\
\hline Lead & 38 & 2.9 & .2 & .9 & .8 \\
\hline Manganese & 38 & 410 & 33.1 & 157 & 135 \\
\hline Nickel & 38 & 1.1 & .1 & .3 & .2 \\
\hline Zinc & 38 & 567 & 103 & 273 & 253 \\
\hline \multicolumn{6}{|c|}{ Hydropsyche cockerelli } \\
\hline Arsenic & 2 & 4.5 & 4.4 & 4.4 & -- \\
\hline Cadmium & 31 & 2.3 & .4 & 1.2 & .9 \\
\hline Chromium & 31 & 3.5 & .4 & 1.6 & 1.6 \\
\hline Copper & 31 & 156 & 30.0 & 59.9 & 50.7 \\
\hline Iron & 31 & 2,500 & 506 & 1,180 & 984 \\
\hline Lead & 31 & 15.0 & 5.1 & 8.6 & 7.7 \\
\hline Manganese & 31 & 1,680 & 549 & 946 & 901 \\
\hline Nickel & 31 & 2.0 & .5 & 1.1 & 1.1 \\
\hline Zinc & 31 & 248 & 134 & 192 & 184 \\
\hline \multicolumn{6}{|c|}{ Hydropsyche morosa group } \\
\hline Cadmium & 6 & 1.3 & 1.1 & 1.2 & 1.2 \\
\hline Chromium & 6 & 2.8 & 1.9 & 2.3 & 2.2 \\
\hline Copper & 6 & 57.4 & 50.2 & 55.2 & 55.8 \\
\hline Iron & 6 & 1,730 & 1,380 & 1,570 & 1,600 \\
\hline Lead & 6 & 10.8 & 7.0 & 8.9 & 9.0 \\
\hline Manganese & 6 & 1,940 & 1,260 & 1,610 & 1,620 \\
\hline Nickel & 6 & 1.7 & 1.3 & 1.5 & 1.5 \\
\hline Zinc & 6 & 250 & 227 & 239 & 240 \\
\hline \multicolumn{6}{|c|}{ Hydropsyche occidentalis } \\
\hline Arsenic & 2 & 4.3 & 4.3 & 4.3 & -- \\
\hline Cadmium & 18 & 2.0 & .4 & 1.1 & 1.1 \\
\hline Chromium & 18 & 8.1 & .4 & 2.4 & 2.3 \\
\hline Copper & 18 & 118 & 13.3 & 54.6 & 54.1 \\
\hline Iron & 18 & 2,060 & 424 & 1,270 & 1,190 \\
\hline Lead & 18 & 14.0 & 2.9 & 9.0 & 8.7 \\
\hline Manganese & 18 & 2,920 & 619 & 1,480 & 1,210 \\
\hline Nickel & 18 & 2.4 & .5 & 1.4 & 1.4 \\
\hline Zinc & 18 & 293 & 157 & 221 & 222 \\
\hline
\end{tabular}


102 Water-Quality, Bed-Sediment, and Biological Data (October 2002 through September 2003) and Statistical Summaries of Data for Streams in the Upper Clark Fork Basin, Montana

Table 24. Statistical summary of biological data for the upper Clark Fork basin, Montana, August 1986 through September 2003-Continued

Constituent $\quad \begin{gathered}\text { Number of } \\ \text { composite } \\ \text { samples }\end{gathered}$ Maximum Minimum Mean $\quad$ Median

12331800--CLARK FORK NEAR DRUMMOND, MONT.-Continued Period of record for biological data: 1986, 1991-2003

Cadmium

Chromium

Copper

Iron

Lead

Manganese

Nickel

Zinc

Arsenic

Cadmium

Chromium

Copper

Iron

Lead

Manganese

Nickel

Zinc

Arsenic

Cadmium

Chromium

Copper

Iron

Lead

Manganese

Nickel

Zinc

Arsenic

Cadmium

Chromium

Copper

Iron

Lead

Manganese

Nickel

Zinc

Arsenic

Cadmium

Chromium

Copper

Iron

Lead

\section{Hydropsyche spp}

$\begin{array}{llcc}1 & -- & -- & 2.6 \\ 0 & -- & -- & -- \\ 1 & -- & -- & 85.0 \\ 1 & -- & -- & 940 \\ 1 & -- & -- & 9.1 \\ 0 & -- & -- & -- \\ 0 & -- & -- & -- \\ 1 & -- & -- & 260\end{array}$

12334510--ROCK CREEK NEAR CLINTON, MONT. Period of record for biological data: 1987, 1991-99, 2001-03

Arctopsyche grandis

$\begin{array}{rcccc}4 & 2.2 & 1.9 & 2.0 & 2.0 \\ 42 & .4 & .06 & .2 & .2 \\ 42 & 2.9 & .5 & 1.2 & 1.0 \\ 42 & 15.7 & 4.7 & 8.6 & 8.5 \\ 42 & 1,090 & 191 & 582 & 506 \\ 42 & 1.1 & .05 & 1.4 & 1.4 \\ 42 & 454 & 113 & 250 & 227 \\ 42 & 1.8 & .2 & .9 & .9 \\ 42 & 189 & 84 & 127 & 130\end{array}$

Claassenia sabulosa

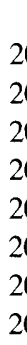

Hydropsyche cockerelli

$\begin{array}{rr}-- & - \\ .3 & <.2 \\ 1.0 & .3 \\ 13.1 & 6.0\end{array}$

825

$<1.1$

266

1.0

99

\section{Hydropsyche occidentalis}

$\begin{array}{rcc}-- & -- & 2.2 \\ .4 & <.3 & 1.2 \\ 2.4 & .9 & 1.6 \\ 17.6 & 5.1 & 10.6 \\ 973 & 520 & 709 \\ 6.0 & 1.2 & 3.0\end{array}$

0

8.5

1.4

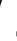

130

$--$

.2

27.9

102

.3

33.6

191

$<.2$

.9

7.4

553

$<1.1$

233

.6

91

.14

1.6

10.2

652 
Table 24. Statistical summary of biological data for the upper Clark Fork basin, Montana, August 1986 through September 2003-Continued

\begin{tabular}{|c|c|c|c|c|c|}
\hline Constituent & $\begin{array}{l}\text { Number of } \\
\text { composite } \\
\text { samples }\end{array}$ & Maximum & Minimum & Mean & Median \\
\hline \multicolumn{6}{|c|}{$\begin{array}{l}\text { 12334510--ROCK CREEK NEAR CLINTON, MONT-CContinued } \\
\text { Period of record for biological data: 1987, 1991-99, 2001-03 }\end{array}$} \\
\hline \multicolumn{6}{|c|}{ Hydropsyche occidentalis - Continued } \\
\hline Manganese & 5 & 295 & 169 & 242 & 262 \\
\hline Nickel & 5 & 1.7 & .6 & 1.2 & 1.4 \\
\hline Zinc & 5 & 144 & 99 & 116 & 117 \\
\hline \multicolumn{6}{|c|}{ Hydropsyche spp. } \\
\hline Cadmium & 3 & .3 & $<.5$ & 1.2 & .2 \\
\hline Chromium & 3 & 2.1 & 1.1 & 1.6 & 1.7 \\
\hline Copper & 3 & 16.2 & 11.6 & 14.3 & 15.0 \\
\hline Iron & 3 & 1,140 & 837 & 1,000 & 1,030 \\
\hline Lead & 3 & $<3.1$ & $<1.8$ & 1 & $<2.9$ \\
\hline Manganese & 3 & 462 & 299 & 399 & 437 \\
\hline Nickel & 3 & 1.3 & .8 & 1.1 & 1.1 \\
\hline Zinc & 3 & 135 & 117 & 126 & 126 \\
\hline
\end{tabular}

12334550--CLARK FORK AT TURAH BRIDGE, NEAR BONNER, MONT. Period of record for biological data: 1986, 1991-2003

Arsenic

Cadmium

Chromium

Copper

Iron

Lead

Manganese

Nickel

Zinc

Arctopsyche grandis

Cadmium

Chromium

Copper

Iron

Lead

Manganese

Nickel

Zinc

Arsenic

Cadmium

Chromium

Copper

Iron

Lead

Manganese

Nickel

Zinc

$\begin{array}{rc} & \text { Arctopsyche grandis } \\ 4 & 5.0 \\ 46 & 2.7 \\ 46 & 4.1 \\ 46 & 125 \\ 46 & 2,870 \\ 46 & 13.2 \\ 46 & 893 \\ 46 & 2.7 \\ 46 & 276 \\ & \text { Claassenia sabulosa }\end{array}$

4.5
.3
.6
20.1
372

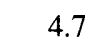

1.2

1.7

1.7
38.8

1.6

324

.4

979

4.5

615

111

1.1

196

.9
1.5
30.4
827
3.8
626
196

2
28
28
28
28
28
28
28

.8

2.5

2.0

79.2

181

1.6

139

.6

283

\section{.8}

.2

.8

1.0

.7

55.7

102

58.6

.2

37.2

.6
76.2

$144^{.1}$

.2

Hydropsyche cockerelli

$\begin{array}{rcccc}3 & 4.1 & 3.7 & 3.9 & 3.9 \\ 31 & 1.8 & .3 & .8 & .7 \\ 31 & 8.0 & .16 & 1.9 & 1.6 \\ 31 & 118 & 26.4 & 47.7 & 42.5 \\ 31 & 2,530 & 566 & 1,210 & 1,090 \\ 31 & 12.1 & 2.2 & 5.2 & 5.0 \\ 31 & 788 & 426 & 596 & 575 \\ 31 & 2.6 & .6 & 1.2 & 1.2 \\ 31 & 228 & 119 & 182 & 180\end{array}$


104 Water-Quality, Bed-Sediment, and Biological Data (October 2002 through September 2003) and Statistical Summaries of Data for Streams in the Upper Clark Fork Basin, Montana

Table 24. Statistical summary of biological data for the upper Clark Fork basin, Montana, August 1986 through September 2003 - Continued

\begin{tabular}{|c|c|c|c|c|c|c|}
\hline & Constituent & $\begin{array}{c}\text { Number of } \\
\text { composite } \\
\text { samples }\end{array}$ & Maximum & Minimum & Mean & Median \\
\hline \multicolumn{7}{|c|}{ 12334550--CLARK FORK AT TURAH BRIDGE, NEAR BONNER, MONT-Continued } \\
\hline \multicolumn{7}{|c|}{ Period of record for biological data: 1986, 1991-2003 } \\
\hline \multicolumn{7}{|c|}{ Hydropsyche morosa group } \\
\hline Cadmium & & 2 & 1.3 & 1.1 & 1.2 & -. \\
\hline Chromium & & 2 & 4.6 & 2.4 & 3.5 & -- \\
\hline Copper & & 2 & 84.1 & 26.8 & 55.4 & -- \\
\hline Iron & & 2 & 1,800 & 986 & 1,390 & -- \\
\hline Lead & & 2 & 6.6 & $<7.8$ & ${ }^{1} 5.2$ & -- \\
\hline Manganese & & 2 & 1,320 & 537 & 928 & -- \\
\hline Nickel & & 2 & 1.7 & 1.3 & 1.5 & -- \\
\hline Zinc & & 2 & 231 & 171 & 201 & -- \\
\hline \multicolumn{7}{|c|}{ Hydropsyche occidentalis } \\
\hline Arsenic & & 1 & -- & -- & 3.6 & -- \\
\hline Cadmium & & 21 & 1.8 & .3 & .9 & .8 \\
\hline Chromium & & 21 & 3.2 & .6 & 1.9 & 1.7 \\
\hline Copper & & 21 & 102 & 27.4 & 49.0 & 43.7 \\
\hline Iron & & 21 & 2,310 & 472 & 1,200 & 1,130 \\
\hline Lead & & 21 & 14.2 & 3.0 & 6.5 & 5.7 \\
\hline Manganese & & 21 & 1,600 & 454 & 807 & 712 \\
\hline Nickel & & 21 & 3.2 & .6 & 1.2 & 1.1 \\
\hline Zinc & & 21 & 416 & 145 & 205 & 194 \\
\hline \multicolumn{7}{|c|}{ Hydropsyche spp. } \\
\hline Cadmium & & 1 & -- & -- & 1.3 & -- \\
\hline Chromium & & 1 & -- & -- & 2.4 & -- \\
\hline Copper & & 1 & -- & -- & 84.1 & -- \\
\hline Iron & & 1 & -- & -- & 1,800 & -- \\
\hline Lead & & 1 & -- & -- & $<7.8$ & -- \\
\hline Manganese & & 1 & -- & -- & 537 & -- \\
\hline Nickel & & 1 & -- & -- & 1.3 & -- \\
\hline Zinc & & 1 & -- & -. & 171 & -. \\
\hline
\end{tabular}

12340000--BLACKFOOT RIVER NEAR BONNER, MONT.

Period of record for biological data: 1986-87, 1991, 1993, 1996, 1998, 2000, 2003

Arsenic
Cadmium
Chromium
Copper
Iron
Lead
Manganese
Nickel
Zinc

Cadmium
Chromium
Copper
Iron
Lead
Manganese

Arctopsyche grandis

$\begin{array}{rc}1 & -- \\ 11 & .4 \\ 5 & 1.8 \\ 11 & 13.4 \\ 11 & 1,230 \\ 11 & 2.1 \\ 5 & 517 \\ 5 & 1.2 \\ 11 & 143\end{array}$

--
$<.1$
.8
9.9
108
.5
286
123
.8

$\begin{array}{cc}2.8 & -- \\ 1.2 & 1.2 \\ 1.3 & 1.2 \\ 12.0 & 12.0 \\ 606 & 617 \\ 1.0 & .6 \\ 413 & 398 \\ 1.0 & 1.0 \\ 135 & 136\end{array}$

Claassenia sabulosa

$\begin{array}{rcrcr}11 & .2 & .1 & .1 & .1 \\ 5 & .9 & .3 & .5 & .5 \\ 11 & 88.5 & 19.0 & 45.2 & 44.0 \\ 11 & 158 & 46.2 & 100 & 99.0 \\ 11 & .6 & .4 & .3 & .4 \\ 5 & 127 & 26.3 & 57.1 & 44.7\end{array}$


Table 24. Statistical summary of biological data for the upper Clark Fork basin, Montana, August 1986 through September 2003-Continued

\begin{tabular}{cccccc}
\hline Constituent & $\begin{array}{c}\text { Number of } \\
\text { composite } \\
\text { samples }\end{array}$ & Maximum & Minimum & Mean & Median \\
\hline
\end{tabular}

12340000--BLACKFOOT RIVER NEAR BONNER, MONT.-Continued

Period of record for biological data: 1986-87, 1991, 1993, 1996, 1998, 2000, 2003

Nickel

Zinc

Arsenic

Cadmium

Chromium

Copper

Iron

Lead

Manganese

Nickel

Zinc

Cadmium

Chromium

Copper

Iron

Lead

Manganese

Nickel

Zinc

Arsenic

Cadmium

Chromium

Copper

Iron

Lead

Manganese

Nickel

Zinc

Arsenic

Cadmium

Chromium

Copper

Iron

Lead

Manganese

Nickel

Zinc

Arsenic

Cadmium
Claassenia sabulosa-Continued

$\begin{array}{rccccc}5 & .3 & .1 & .2 & .2 \\ 11 & 329 & 117^{.2} & 209 & 194^{.2}\end{array}$

Hydropsyche occidentalis

$\begin{array}{rcccc}1 & -- & -- & 3.2 & -- \\ 13 & .5 & .1 & .2 & .2 \\ 13 & 2.7 & .8 & 1.8 & 1.7 \\ 13 & 20.6 & 12.0 & 14.4 & 14.4 \\ 13 & 1,930 & 1,060 & 1,410 & 1,470 \\ 13 & 1.9 & .8 & 1.3 & 1.3 \\ 13 & 577 & 414 & 480 & 466 \\ 13 & 1.8 & .9 & 1.3 & 1.2 \\ 13 & 150 & 116 & 135 & 130\end{array}$

12340500--CLARK FORK ABOVE MISSOULA, MONT.

Period of record for biological data: 1997-2003

$\begin{array}{lccc}-- & -- & .6 & -- \\ -- & -- & 1.6 & -- \\ -- & -- & 13.9 & -- \\ -- & -- & 1,140 & -- \\ -- & -- & 2.9 & -- \\ -- & -- & 525 & -- \\ -- & -- & 2.8 & -- \\ -- & -- & 132 & --\end{array}$

Arctopsyche grandis

$\begin{array}{cc}3 & 4.5 \\ 22 & 1.8 \\ 22 & 3.4 \\ 22 & 77.6 \\ 22 & 2,340 \\ 22 & 6.8 \\ 22 & 1,400 \\ 22 & 2.0 \\ 22 & 260\end{array}$

4.0
.4
.6
19.5
476
1.2
476
.5
133

$\begin{array}{cc}4.2 & 4.2 \\ 7 & .6 \\ 1.6 & 1.5 \\ 34.4 & 28.2 \\ 1,030 & 953 \\ 3.7 & 3.3 \\ 902 & 887 \\ 1.2 & 1.0 \\ 189 & 195\end{array}$

Claassenia sabulosa

\begin{tabular}{|c|c|c|c|c|}
\hline 2 & 1.1 & .7 & .9 & -- \\
\hline 11 & 2.0 & .2 & .7 & .4 \\
\hline 11 & 1.1 & .3 & .7 & .7 \\
\hline 11 & 71.7 & 33.0 & 48.9 & 46.0 \\
\hline 11 & 402 & 95.3 & 247 & 246 \\
\hline 11 & 3.1 & .5 & ${ }_{1.3}$ & ${ }^{1} 1.2$ \\
\hline 11 & 683 & 75.2 & 261 & 270 \\
\hline 11 & $<.4$ & $<.3$ & ${ }^{1} .4$ & ${ }^{1} .4$ \\
\hline 11 & 363 & 191 & 268 & 250 \\
\hline \multicolumn{5}{|c|}{ Hydropsyche cockerelli } \\
\hline 2 & 6.5 & 6.4 & 6.4 & -- \\
\hline 11 & 1.3 & .4 & .8 & .8 \\
\hline
\end{tabular}


106 Water-Quality, Bed-Sediment, and Biological Data (October 2002 through September 2003) and Statistical Summaries of Data for Streams in the Upper Clark Fork Basin, Montana

Table 24. Statistical summary of biological data for the upper Clark Fork basin, Montana, August 1986 through September 2003-Continued

\begin{tabular}{|c|c|c|c|c|c|}
\hline Constituent & $\begin{array}{c}\text { Number of } \\
\text { composite } \\
\text { samples }\end{array}$ & Maximum & Minimum & Mean & Median \\
\hline \multicolumn{6}{|c|}{ 12340500--CLARK FORK ABOVE MISSOULA, MONT-Continued } \\
\hline \multicolumn{6}{|c|}{ Hydropsyche cockerelli-Continued } \\
\hline Chromium & 11 & 6.0 & 1.8 & 3.3 & 3.2 \\
\hline Copper & 11 & 96.1 & 29.9 & 57.2 & 46.0 \\
\hline Iron & 11 & 3,590 & 1,400 & 2,160 & 2,070 \\
\hline Lead & 11 & 10.0 & 4.2 & 6.2 & 5.7 \\
\hline Manganese & 11 & 1,890 & 781 & 1,190 & 1,130 \\
\hline Nickel & 11 & 2.4 & 1.4 & 1.9 & 1.9 \\
\hline Zinc & 11 & 237 & 156 & 197 & 194 \\
\hline \multicolumn{6}{|c|}{ Hydropsyche occidentalis } \\
\hline Arsenic & 2 & 6.2 & 6.2 & 6.2 & -- \\
\hline Cadmium & 8 & 1.1 & .4 & .7 & .6 \\
\hline Chromium & 8 & 5.5 & 2.1 & 3.4 & 3.0 \\
\hline Copper & 8 & 76.5 & 30.3 & 51.4 & 52.9 \\
\hline Iron & 8 & 2,400 & 1,450 & 2,060 & 2,220 \\
\hline Lead & 8 & 10.2 & 4.0 & 6.8 & 6.6 \\
\hline Manganese & 8 & 2,460 & 939 & 1,840 & 1,950 \\
\hline Nickel & 8 & 2.4 & 1.6 & 2.0 & 2.2 \\
\hline Zinc & 8 & 257 & 192 & 227 & 230 \\
\hline \multicolumn{6}{|c|}{ 12353000--CLARK FORK BELOW MISSOULA, MONT. ${ }^{2}$} \\
\hline \multicolumn{6}{|c|}{ Period of record for biological data: $1986,1990-2003$} \\
\hline \multicolumn{6}{|c|}{ Arctopsyche grandis } \\
\hline Arsenic & 3 & 2.6 & 2.4 & 2.5 & 2.5 \\
\hline Cadmium & 28 & 1.5 & .2 & .7 & .6 \\
\hline Chromium & 28 & 2.7 & .5 & 1.3 & 1.4 \\
\hline Copper & 28 & 38.0 & 9.4 & 21.1 & 19.8 \\
\hline Iron & 28 & 1,590 & 343 & 804 & 718 \\
\hline Lead & 28 & 3.9 & .9 & 1.9 & 1.8 \\
\hline Manganese & 28 & 1,090 & 511 & 701 & 675 \\
\hline Nickel & 28 & 1.6 & .4 & .9 & .9 \\
\hline Zinc & 28 & 217 & 106 & 152 & 148 \\
\hline \multicolumn{6}{|c|}{ Claassenia sabulosa } \\
\hline Arsenic & 2 & .8 & .8 & .8 & -- \\
\hline Cadmium & 41 & 1.3 & .1 & .5 & .4 \\
\hline Chromium & 41 & 1.2 & .05 & .5 & .5 \\
\hline Copper & 41 & 74.8 & 31.1 & 48.1 & 46.9 \\
\hline Iron & 41 & 239 & 66.6 & 111 & 103 \\
\hline Lead & 41 & 1.3 & .1 & .4 & .3 \\
\hline Manganese & 41 & 168 & 48.9 & 102 & 99.0 \\
\hline Nickel & 41 & .3 & .1 & .2 & .2 \\
\hline Zinc & 41 & 286 & 146 & 215 & 210 \\
\hline \multicolumn{6}{|c|}{ Hydropsyche cockerelli } \\
\hline Arsenic & 5 & 2.4 & 2.2 & 2.4 & 2.4 \\
\hline Cadmium & 43 & 1.1 & .2 & .5 & .5 \\
\hline Chromium & 43 & 3.4 & .3 & 1.8 & 1.8 \\
\hline Copper & 43 & 54.1 & 12.4 & 30.1 & 27.5 \\
\hline Iron & 43 & 2,220 & 584 & 1,290 & 1,320 \\
\hline Lead & 43 & 6.6 & 1.2 & 2.5 & 2.3 \\
\hline Manganese & 43 & 1,210 & 353 & 752 & 684 \\
\hline
\end{tabular}


Table 24. Statistical summary of biological data for the upper Clark Fork basin, Montana, August 1986 through September 2003-Continued

\begin{tabular}{|c|c|c|c|c|c|}
\hline Constituent & $\begin{array}{l}\text { Number of } \\
\text { composite } \\
\text { samples }\end{array}$ & Maximum & Minimum & Mean & Median \\
\hline \multicolumn{6}{|c|}{ 12353000--CLARK FORK BELOW MISSOULA, MONT ${ }^{2}$ - Continued } \\
\hline \multicolumn{6}{|c|}{ Period of record for biological data: $1986,1990-2003$} \\
\hline \multicolumn{6}{|c|}{ Hydropsyche cockerelli-Continued } \\
\hline Nickel & 43 & 1.9 & .5 & 1.2 & 1.3 \\
\hline Zinc & 43 & 187 & 77.4 & 142 & 147 \\
\hline \multicolumn{6}{|c|}{ Hydropsyche occidentalis } \\
\hline Arsenic & 1 & -- & -- & 2.5 & -- \\
\hline Cadmium & 17 & 1.1 & .1 & .4 & .3 \\
\hline Chromium & 17 & 3.5 & .1 & 1.4 & 1.5 \\
\hline Copper & 17 & 38.2 & 13.5 & 23.8 & 20.9 \\
\hline Iron & 17 & 1,420 & 482 & 941 & 907 \\
\hline Lead & 17 & 4.2 & .7 & 2.1 & 1.9 \\
\hline Manganese & 17 & 1,460 & 491 & 841 & 812 \\
\hline Nickel & 17 & 2.2 & .5 & 1.0 & .9 \\
\hline Zinc & 17 & 193 & 112 & 143 & 144 \\
\hline \multicolumn{6}{|c|}{ Hydropsyche spp. } \\
\hline Cadmium & 1 & -- & -- & .5 & -- \\
\hline Chromium & 1 & -- & -- & .8 & -- \\
\hline Copper & 1 & -- & -- & 20.8 & -- \\
\hline Iron & 1 & -- & -. & 894 & -- \\
\hline Lead & 1 & -- & -- & 1.1 & -- \\
\hline Manganese & 1 & -- & -- & 756 & -- \\
\hline Nickel & 1 & -- & -- & 1.1 & -- \\
\hline Zinc & 1 & -- & -- & 124 & -- \\
\hline
\end{tabular}

${ }^{1}$ Values determined by arbitrarily substituting one-half of the analytical detection limit for censored $(<)$ values when both uncensored and censored values were used in determining the mean and median. When all data were less than the analytical detection limit, the median was determined by ranking the censored values in order of detection. No mean is reported when all values were below the analytical detection limit.

${ }^{2}$ Samples collected about 30 miles downstream from streamflow-gaging station to conform to previous sampling location. 